\title{
Improving feed
}

efficiency in pigs.

\section{Bridging genetics and nutrition}

Lisanne M.G. Verschuren

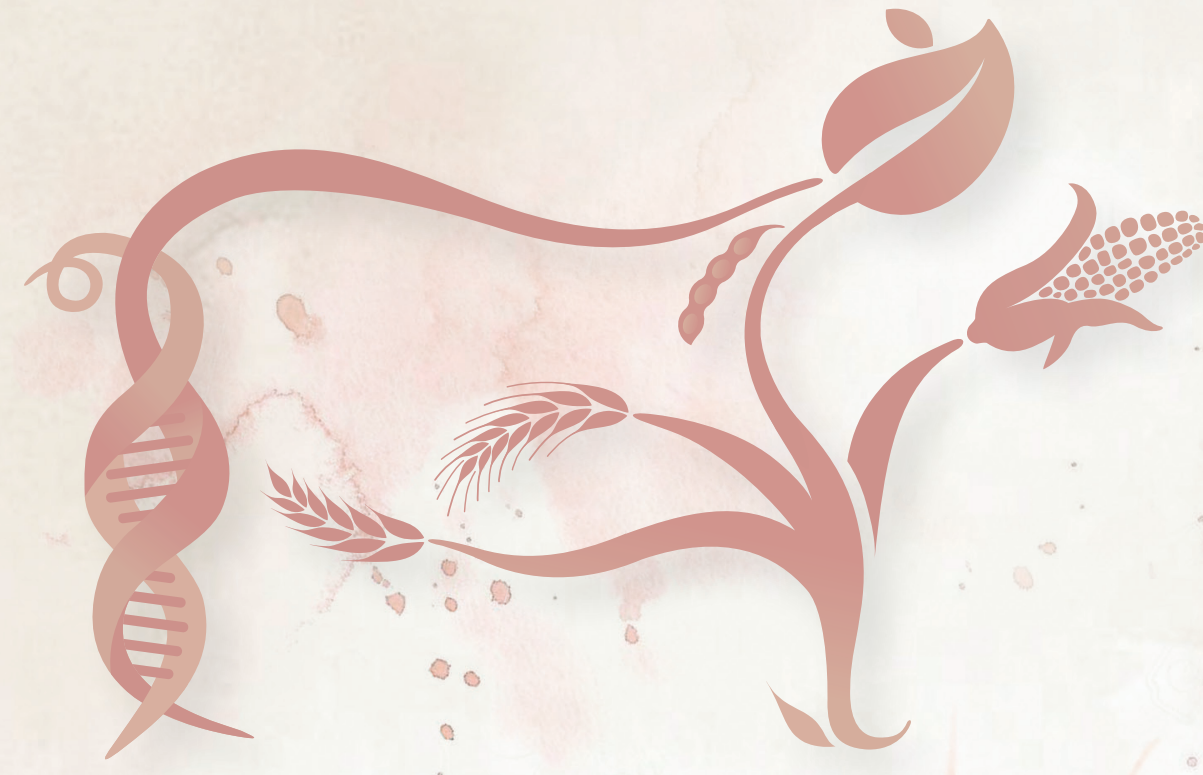

0

8 



\section{Propositions}

1. Understanding biology is essential to further increase genetic progress for feed efficiency in pigs.

(this thesis)

2. Measuring biological parameters in pigs is the only way forward to continue improving nutrient utilization with feeding strategies.

(this thesis)

3. Protection of raw data produced by equipment used in research hampers scientific and technological progress.

4. The general public should be allowed to vote on topics to be investigated in science.

5. World aspirations must prevail over nationality when forming friendships.

6. For human society it is more important to invest time in social interactions with people thinking differently than with like-minded thinkers.

Propositions belonging to the thesis, entitled:

Improving feed efficiency in pigs: bridging genetics and nutrition

Lisanne Verschuren

Wageningen, 10 September

2021 



\section{Improving feed efficiency in pigs: bridging genetics and nutrition}

Lisanne M.G. Verschuren 


\section{Thesis committee}

\section{Promotor}

Dr M.P.L. Calus

Associate professor, Animal Breeding and Genomics Group

Wageningen University \& Research

\section{Co-promotors}

Dr A.J.M. Jansman

Senior researcher, Animal Nutrition

Wageningen University \& Research

Dr J. van Milgen

Senior researcher, National Research Institute for Agriculture, Food and

Environment

L'institute Agro

Dr R. Bergsma

Senior Geneticist

Topigs Norsvin Research Center

\section{Other members}

Prof. Dr J. Bennewitz, University of Hohenheim, Germany

Prof. Dr J.M.J. Rebel, Wageningen University \& Research

Prof. Dr L. Montagne, Agrocampus Ouest, France

Prof. Dr M. Kleerebezem, Wageningen University \& Research

This research was conducted under the auspices of the Graduate School of Ecologie, Géosciences, Agronomie et Alimentation, France, and the Graduate School of Wageningen Institute of Animal Sciences, The Netherlands, and as part of a joint PhD programme. 


\title{
Improving feed efficiency in pigs: bridging genetics and nutrition
}

\author{
Lisanne M.G. Verschuren
}

\begin{abstract}
Thesis
submitted in fulfilment of the requirements for the joint degree of doctor between L'institut Agro

by the authority of the Head of Ecole doctorale Ecologie, Géosciences, Agronomie et Alimentation and Wageningen University

by the authority of the Rector Magnificus

Prof. dr. A.P.J. Mol,

in the presence of the
\end{abstract}

Thesis Committee appointed by the Academic Board at Wageningen University and

the Scientific Council at L'institut Agro

to be defended in public

on Friday September 10, 2021

at 4 p.m. in the Aula 
Lisanne M.G. Verschuren.

Improving feed efficiency in pigs: bridging genetics and nutrition. 190 pages.

Joint PhD thesis, L'institut Agro, France and Wageningen University, the Netherlands (2021)

With references, with summaries in English and French

ISBN 978-94-6395-889-9

DOI: https://doi.org/10.18174/549769 


\section{Abstract}

Verschuren, L.M.G. (2021). Improving feed efficiency in pigs: bridging genetics and nutrition. PhD thesis, Wageningen University, the Netherlands

The efficiency of turning a pig diet into a human edible product, commonly called feed efficiency, is key to decrease environmental pollution of the pig industry. In this thesis I bridge the genetics and nutrition expertise fields in pig production. I investigate traits explaining variation in individual feed efficiency in pigs, and their potential to develop more effective nutritional and breeding strategies to improve feed efficiency. This thesis shows that there is variation in feed efficiency traits of grower-finisher pigs associated with variation in faecal digestibility values, independent of variation in feed intake. Also the variation in faecal microbiota composition is shown to be associated with variation in feed efficiency, but the relationship depends on diet and sex. Faecal microbiota is associated to variation in faecal nutrient digestibility as well, and can even be used to predict faecal nutrient digestibility values. Further, this thesis describes the phenotypic variation for individual nitrogen and amino acid efficiency and shows that nitrogen efficiency estimated based on feed intake and growth curves is heritable. The faecal microbiota and serum metabolites are found to be heritable too, and the research described in this thesis shows that there is substantial overlap between variation in feed efficiency explained by the host genome, faecal microbiota composition, and serum metabolite profiles. Hence, in this thesis I show that variation in faecal nutrient digestibility, faecal microbiota composition, serum metabolite profiles, and nitrogen efficiency are associated with variation in feed efficiency, and that there is a genetic background. The characteristics investigated in this thesis could be used to improve feed efficiency in pigs by means of breeding, but also to predict individual growerfinisher performance and aid nutritionist in precision feeding concepts. Likewise, pig nutritionists could help breeders in developing a feeding strategy to express more genetic variation. The results in this thesis support that bridging the gap between genetics and nutrition is the way forward to further improve feed efficiency in grower-finisher pigs. 



\section{Contents}

5 Abstract

$9 \quad 1-$ General introduction

212 - Variation in faecal digestibility values related to feed efficiency traits of grower-finisher pigs

413 - Fecal microbial composition associated with variation in feed efficiency in pigs depends on diet and sex

634 - Prediction of nutrient digestibility in grower-finisher pigs based on faecal microbiota composition

835 - Between animal variation in dietary nitrogen and amino acid efficiency in grower-finisher pigs

1016 -Combining faecal microbiome, serum metabolome, and host genome for phenotypic prediction of feed efficiency in grower-finisher pigs

1317 -General discussion

173 Summary

177 Résumé

181 Curriculum Vitae

187 Acknowledgements 

1

General introduction 



\subsection{Introduction}

Wednesday the $29^{\text {th }}$ of May 2019 the highest administrative court of the Netherlands ruled that the Dutch nitrogen legislation was in conflict with the European Habitats Directive. As a result, construction work was halted, the maximum speed limit was changed from 130 to $100 \mathrm{~km} / \mathrm{h}$, and it was suggested that livestock numbers should be decreased. Dutch farmers reacted to this last suggestion by organising a protest on the first of October 2019 and tractors flooded The Hague. All of this because nitrogen emission in the Netherlands exceeded maximum allowed levels from a legislation perspective. Free atmospheric nitrogen is not a problem, however, once nitrogen is oxidized it becomes a pollutant to the environment (Bodirsky et al., 2014). Also when nitrogen is leached to the environment it can lead to eutrophication (Bodirsky et al., 2014). Nitrogen emission, however, is not only a problem in the Netherlands. Worldwide nitrogen emission already exceeds global critical thresholds and will most likely continue to rise, even when mitigation strategies including efficient livestock management are applied (Bodirsky et al., 2014). Not only nitrogen is a potential environmental polluter, also carbon dioxide and methane released by livestock production are important players in climate change (Steinfeld et al., 2006). A small piece of the solution in reducing nitrogen, carbon dioxide and methane pollution could come from the pig industry. Pigs are ideal animals to convert products inedible for humans to valuable protein for human consumption (Zijlstra and Beltranena, 2013). But even if pigs can use human inedible products, any inefficiency of turning dietary nutrients into body tissue of pigs leads to environmental pollution. Thus, efficiency of turning a pig diet into a human edible product, is key.

In this chapter I will first explain the current frameworks of feed efficiency and which efforts have been made to improve feed efficiency. Hereafter I take you on a tour through the pig body to explain important biological processes related to feed efficiency. The tour is followed by a description of the goal of my thesis. 


\section{General introduction}

\subsection{Feed efficiency}

Feed efficiency is a term used quite often in pig production to indicate the efficiency of turning pig feed into a pork product. However, feed efficiency is not always defined and calculated in a similar way. To start with geographical differences, in Europe they are used to a term called feed conversion ratio (FCR), which is calculated as feed intake over bodyweight gain, whereas in the America's they are more used to the term gain:feed ("gain-to-feed"), which is calculated as bodyweight gain over feed intake. Both FCR and gain:feed are used to describe feed efficiency, but sometimes the traits underlying FCR and gain:feed, average daily gain (ADG) and average daily feed intake (ADG), are themselves also called feed efficiency traits. Another commonly used term to describe feed efficiency of grower-finisher pigs is residual feed intake (RFI). In general, there are two ways RFI is calculated. One way to calculate RFI is compared to other pigs in the same cohort or experiment. In a statistical model, average daily feed intake (ADFI) is corrected for the ADG, back fat thickness (BF), age at start of the period, and weight at start and end of the period. The residual error of this statistical model is the RFI (Cai et al., 2008), where the mean RFI is zero and positive RFI values are considered unfavourable. Another way to calculate RFI is by using metabolic relationships. The metabolizable energy consumption of an individual pigs is corrected for the energy required for maintenance, protein deposition and lipid deposition as estimated based on metabolic relationships (Bergsma et al., 2013). The latter method has also been termed residual energy intake (REI).

The composition of the feed and the eventual pork varies a lot. Calculating feed efficiency as FCR or gain:feed completely ignores the differences in nutrient composition of feeds between batches and body composition between pigs, hence making it difficult to compare values across studies. Also calculating RFI according to the method of Cai et al. (2008) is only based on performance differences between pigs and does not account for differences in nutrient composition of the diet. Calculating RFI or REl as suggested by Bergsma et al. (2013) does take into account the differences in energy value between diets, but by calculating energy required for maintenance, protein deposition, and lipid deposition based on fixed metabolic relationships the differences between pigs in efficiency of these metabolic processes are ignored. Hence, all ways to calculate feed efficiency have their limitations, and it is important to consider not only the calculated values for the traits but also to consider underlying traits such as ADG, ADFI, and BF. 
In the pig industry efforts have been made to improve feed efficiency. In this thesis I will focus on two important players to improve feed efficiency in the pig industry: the pig breeders and the feed producers. In pig breeding existing variation between animals is used to select candidates for the next generation. With this selection of pigs that have favourable characteristics, called traits, the average of the total population will be improved for those traits. Pig breeding significantly increased leanness and ADG over the years, resulting in improved feed efficiency (Knap and Wang, 2012). In pig nutrition, diets are formulated using different dietary ingredients to balance nutrient supply with the nutrient requirements of pigs. In order to do so the nutrient composition and ileal or faecal digestibility of nutrients in feed ingredients have to be known. Also, the nutritional requirements of pigs should be known to improve the feed efficiency of animals. Nutritional requirements of pigs are updated regularly to be able to formulate diets that more closely fit the pig's nutrient requirements (Van der Peet-Schwering and Bikker, 2018). More recently, precision livestock feeding concepts are further developed to more closely fit the individual requirements of a pig with dietary nutrient supply and hence reduce nutrient losses due to inefficiency (Pomar and Remus, 2019). Even though both genetics and nutrition fields separately improved feed efficiency, differences between diets in cohorts of selection candidates are normally just treated as a nuisance parameter in models by geneticist, whereas differences between pigs in their nutrient requirement are a nuisance in diet formulation by nutritionist. The reality is that neither the diets nor the pigs are the same. In this thesis I will try to bridge this gap between genetics and nutrition in order to improve feed efficiency in grower-finisher pigs.

\subsection{A tour through the pig's body}

All commonly used descriptions of feed efficiency as explained above still simplify biological processes underlying the conversion of dietary nutrients into different tissues of the pig's body. To get a better feel on this complex process we go on a tour through the pig body.

\subsubsection{Feed}

The tour actually begins outside the pig's body, it starts at the feed. Feed produced for pig production is composed of different ingredients. Most often the main dietary ingredients to produce a pig's diet are ingredients that are readily available in the surroundings of the feed manufacturer. For example, in the USA the main dietary ingredients for pig diets are corn and soybean meal (Zijlstra and Beltranena, 2013), 


\section{General introduction}

as those can easily be grown on the vast fields in the climate of the USA where most pigs are housed. In contrast, in Europe the pigs diets often contain ingredients such as wheat, barley and co-products (Zijlstra and Beltranena, 2013), as those are products that on average grow well in the European climate. The dietary ingredients all have a nutrient composition, of which amino acids (AA), starch, fibre, and fat are the main nutrients. The fraction of the sum of all AA in the diet is called (crude) protein. All nutrients have an energetic value which combined determine the energy content of the diet. During diet formulation, inclusion levels of dietary ingredients with known nutrient composition are determined so that a certain nutrient composition of the diet can be reached, while minimizing cost price of the diet. The preferred nutrient composition of a pig's diet depends on the aimed performance of the pigs, housing conditions, immune status, age, breed and a number of other factors.

\subsubsection{Gastrointestinal tract}

After a pig consumes its diet, the diet and its nutrients enter the stomach of the pig and is now called digesta. The digesta stays in the acidic stomach of the pig for 0.6 to 4.1 hours, with lower retention times when the diet contains more soluble nutrients and higher retention times when the pig consumes more feed (Schop et al., 2019). Liquid fractions leave the stomach easier than solid fractions (Schop et al., 2019). When digesta leaves the stomach, it enters the first part of the small intestine, named the duodenum. In the duodenum the low $\mathrm{pH}$ of the digesta is neutralized and pancreatic and bile juices are added to the digesta. Pancreatic juices contain the digestive enzymes that help break down protein to AA, mono, di, and tri-peptides, starch and sucrose to glucose and fructose, and fat to free fatty acids and monoglycerides. Bile juices contain salts to emulsify the fats so that they can be absorbed across the intestinal wall as well. Secretion of pancreatic and bile juices is amongst others affected by the digesta composition, as a high fibre diet resulted in higher pancreatic and bile juice secretion in a $50 \mathrm{~kg}$ pigs (Wenk, 2001). The digesta moves on to the jejunum where small peptides, AAs, and sugars are absorbed across the intestinal tissue into the blood stream, and free fatty acids and monoglycerides are absorbed into the lymph system. There are differences between pigs in the absorption of some nutrients, as gene expression of sodium/glucose co-transporter 1 in tissue of the jejunum was lower in high RFI pigs compared to low RFI pigs, but other nutrient transporters were not different between the RFI groups (Vigors et al., 2016b). In addition, the intestinal brush border produces enzymes and the sucraseisomaltase gene expression was lower in high RFI pigs, but other enzyme gene expressions were similar to low RFI pigs (Vigors et al., 2016b). The absorption of 
nutrients is enhanced by the presence of villi, which are finger like structures on the inside of the jejunum that enlarge the surface area with which the nutrients are in contact with the intestinal tissue. From the jejunum the digesta moves to the ileum, where villi are also present and further absorption of nutrients goes on. The total duration of the digesta in the small intestine ranges from 1.9 to 2.8 hours (Schop et al., 2019).

After the ileum, part of the digesta flows into the caecum, which is a large sac that houses large numbers of microbes. The whole gastro-intestinal tract is colonized by microbiota, and microbial composition differs between intestinal locations and between digesta and mucosa (Looft et al., 2014). Intestinal microbes use the undigested nutrients coming from the small intestine for fermentation, which results in, amongst others, short chain fatty acids (SCFA). Caecal microbe composition and colonic SCFA concentrations differ between high and low RFI pigs (Vigors et al., 2016b), and SCFA concentration and composition differs in different segments of the intestines (Brestenský et al., 2017). SCFA are absorbed across the intestinal wall of the pig and enter the blood stream. Microbiota are more than just bacteria, they also consist of protozoa, fungi and archaea, which all want their share of nutrients to grow and multiply (Paterson et al., 2017). Some by-products of fermentation of a certain microbe is substrate for another microbe, and there is competition amongst microbes for feed in the gastro-intestinal tract of the pigs (Coyte and RakoffNahoum, 2019). Ileal and caecal digesta enter the colon, where microbial fermentation continues and the water of the digesta is reabsorbed into the pig's body. The thickened digesta with undigested and unfermented nutrients and death and alive microbes leave the body through the rectum in the form of faeces. The difference between nutrients consumed in the feed and excreted in the faeces is called the faecal digestibility of a nutrient. Faecal nutrient digestibility and microbiota composition is highly dependent on age of the pig (Le Goff and Noblet, 2001; Kim et al., 2011).

\subsubsection{Blood}

In the small intestine AA and sugar molecules, and in the large intestine SCFA are absorbed and transferred to the bloodstream. Once those molecules enter the bloodstream they are called blood metabolites. Metabolite are "the biochemical substrates or products of enzymatic reactions" (Fontanesi, 2016). The blood running through the tissue of the gastro-intestinal tract is called portal blood and runs through the portal veins to the liver. In the liver metabolic processes occur to balance the body's need. After feed consumption there is a rapid increase in net portal 


\section{General introduction}

glucose flux, which slowly decreases in the period after a meal (Ingerslev et al., 2014). A surplus of glucose is stored in the liver in the form of glycogen and when blood glucose levels are too low, a shortage of glucose is solved by gluconeogenesis after utilizing glycogen storage. Gluconeogenesis is a chemical process in which glucose is produced from AA, fatty acids or lactate. There are differences between pigs in the liver processes, as divergent genetic selection for RFI resulted in pig lines which had different lipogenic, protein catabolizing and protein synthesising enzyme activities in the liver (Le Naou et al., 2012). From the liver the metabolites are transported by the blood throughout the body to all different tissues.

\subsubsection{Body tissue}

From the perspective of using pigs for meat production, two tissues are important: muscle and fat. Muscle tissue exists out of muscle fibres, which are long cells that stretch the whole length of a muscle. At birth the number of muscle fibres is fixed (Wigmore and Stickland, 1983), and when the protein mass of a pig grows, the size of the muscle fibre enlarges while the number of fibres stays the same. Muscle fibres need $A A$ to grow, as the $A A$ are combined to form muscle protein. The $A A$ supply to muscle tissue should be balancing the $A A$ need of muscle tissue, although the $A A$ composition of muscle in pigs can change in response to AA availability (Remus et al., 2020). Even if a pig is not growing, muscles are continuously breaking down their protein and rebuilding it, which is called turnover. The protein synthesis over protein degradation is on average approximately 1.18, and does not differ between pigs divergent in RFI (Hewitt et al., 2020). Building muscle protein cost energy in the form of adenosine triphosphate (ATP), which can be produced from glucose in the Krebs cycle. This glucose can come from the bloodstream, or can come from the muscle glycogen storage. If insufficient $A A$ are available or essential $A A$ are present in an inappropriate ratio, muscle growth is hampered. If insufficient energy in the form of glucose is present to deposit protein, glucose is formed from AA or fatty acids, mainly in the liver but also in small amounts in the muscle tissue itself. Pigs can differ in their metabolism, as pigs divergently selected for RFI have different enzyme activities related to energy catabolism (Le Naou et al., 2012). A shortage of energy can also be solved by using the pig's fat reserves. In moments of energy surplus energy is stored in adipocytes in the form of triglycerides. Both the number and size of adipocytes grows with the fat reserve needed, as shown by results of pigs divergent in RFI (Le Naou et al., 2012). When energy is mobilized, triglycerides are broken down to fatty acids and glycerol and those molecules enter the bloodstream, where they can be used as fuel for gluconeogenesis. In short, AA are used for muscle growth, which costs energy, and a surplus of energy results in growth of fat tissue. 


\subsubsection{Genetics}

These biological processes in the body are under strict control of the genetic makeup of pigs. The genetic code is like a blue print to build a house, it contains all information on how the animal should be build. Every cell in the body of a pig has the exact same code on the DNA helix whirled up in a total of 19 pairs of chromosomes. On these chromosomes lie the genes, pieces of DNA code that determine functions and activity of cells in the body. Even though the DNA code is exactly the same, cells have differentiated functions, which is determined by the onand of switching of genes in the pig embryo. If a cell multiplies, the pre-set function of the cell maintains, and only pluripotent stem cells can grow into any type of cell. To result in messages for cells to start certain biological processes the DNA code is transcribed to form a single stranded copy, called mRNA, and based on that code proteins are built in the cells. These proteins are then used in chemical processes, like enzymes, become part of building blocks of the cell, like actin in the cytoskeleton, or transported out of the cell to neighbouring cells or the bloodstream. When and if a piece of DNA code should be transcribed is decided in a cascade of signals coming from within the cell, or from neighbouring cells or even anywhere else in the body. Metabolites coming from the diet, produced in nutrient metabolism, or produced by microbes in the gastro-intestinal tract could possibly influence the transcription of certain pieces of DNA (Zelezniak et al., 2014).

\subsubsection{A network of processes}

Enzymatic nutrient digestion by the pig, nutrient fermentation by microbes, metabolization of the digestive and fermentative products and the pig's genetic make-up are all related to each other. Figure 1.1 shows a schematic summary of the relationships, where nutrient flows are given by solid arrows, and control by dashed arrows. Nowadays a multitude of technology is available to get a better insight in biological processes and quantify those biological processes. Especially the omics techniques have quickly gained popularity in the life sciences. Genomics is routinely used in animal breeding (Knol et al., 2016). In genomics for animal breeding, variation between pigs in markers on the DNA is measured and used to estimate the relatedness between individual pigs. Two pigs with precisely the same variants of all markers have a genomic relationship of one, whereas pigs with no similar variants have a genomic relationship of zero. Based on the relatedness between pigs, performance can be predicted. Genomics techniques are also used to identify microbes in the gastro-intestinal tract. The identification of microbes is moving from targeting just a small part of the DNA (16S shotgun) to whole genome sequencing 


\section{General introduction}

(Ranjan et al., 2016). Using faecal microbiota information has been suggested as a means to improve efficiency in pigs with breeding (Maltecca et al., 2020). Metabolomics, an analysis in which metabolites are measured in biological samples, is suggested as a promising method to refine and improve current traits or become new traits for animal breeding (Fontanesi, 2016). In my thesis I will use the previously described techniques combined with more traditional techniques to quantify biological processes in the pigs likely related to feed efficiency. I will use genomics for pig and faecal microbiota DNA analysis, metabolomics for serum metabolite analysis, and wet chemistry analysis for faecal nutrient digestion. The relationships between all the biological processes is also looked at in this thesis.

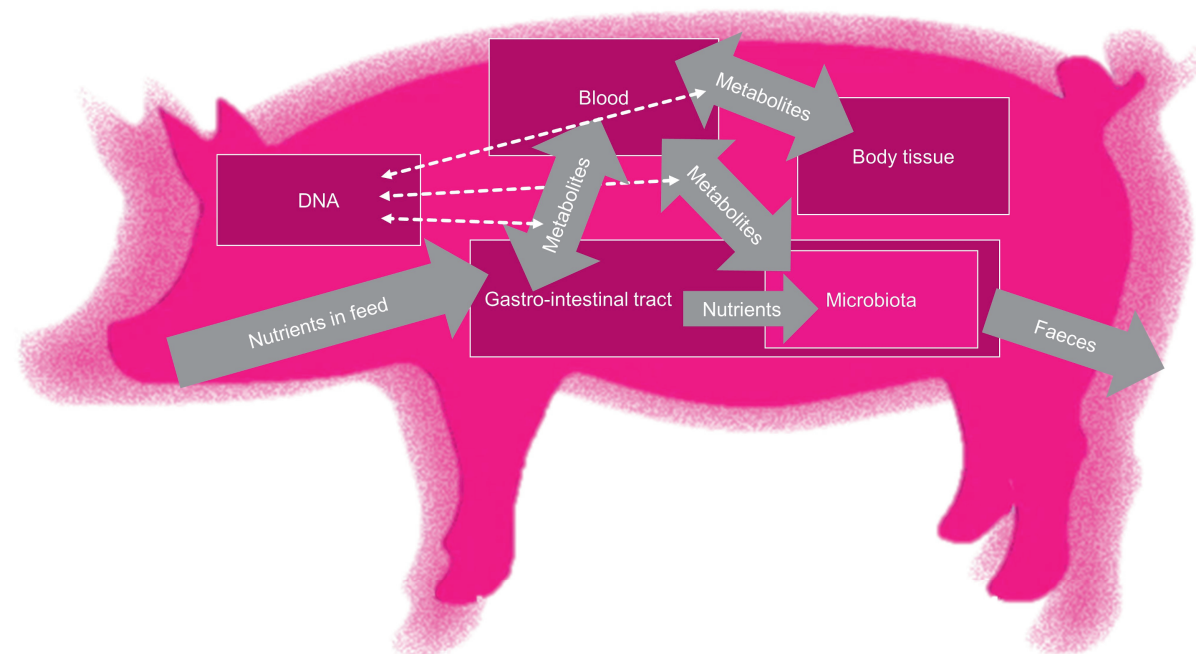

Figure 1.1 Overview of flows of nutrients (solid arrows) and their control (dashed arrows) in the pig's body. 


\subsection{Thesis outline}

The main objective of my research is to investigate traits explaining variation in individual feed efficiency in pigs, and their potential to develop more effective nutritional and breeding strategies to improve feed efficiency. First, I investigate which part of the variation in individual feed efficiency is explained by variation in faecal nutrient digestibility, the faecal microbiome, and nutrient metabolism. Next, I investigate and quantify the relationship between faecal nutrient digestibility, faecal microbiome, nutrient metabolism, and host genetics. Chapter $\mathbf{2}$ describes the variation in feed efficiency traits of grower-finishers pigs associated with the variation in faecal digestibility values, independent of variation in feed intake. Chapter $\mathbf{3}$ investigates the association between feed efficiency and faecal microbial composition in commercial grower-finisher pigs. In addition, two factors affecting feed efficiency are investigated for their effect on the faecal microbiome: diet composition and sex. Chapter 4 estimates the proportion of phenotypic variance in faecal digestibility explained by differences in individual faecal microbiota composition. In addition, the accuracy of predicting faecal digestibility based on microbiota composition is evaluated. Chapter 5 describes the phenotypic variation in nitrogen and amino acid efficiency among grower-finishers pigs based on feed intake and growth curves, and estimates the heritability of nitrogen efficiency and its genetic correlation with feed efficiency traits. Chapter 6 investigates the contribution of host genome, faecal microbiota composition, and concentrations of serum metabolites to variation in feed efficiency traits. The proportion of phenotypic variance in feed efficiency traits associated with microbiota and metabolite profiles is identified, the accuracy of prediction of feed efficiency phenotypes using microbiota and metabolite profiles is evaluated, and the heritability of the faecal microbiome and serum metabolome is estimated to investigate their relationship with the host genome. Finally, I will discuss the implications of the findings described in this thesis for the pig breeding and feeding industry in chapter 7, and I will make several suggestions to bridge the gap between genetics and nutrition in order to improve feed efficiency of the pig industry. 



\section{2}

\section{Variation in faecal digestibility values related to feed efficiency traits of grower-finisher pigs}

L.M.G. Verschuren ${ }^{1,2,3}$, D. Schokker ${ }^{2}$, R. Bergsma ${ }^{1}$, J. van Milgen ${ }^{3}$, F. Molist ${ }^{4}$, M.P.L. Calus ${ }^{5}$, A.J.M. Jansman ${ }^{2}$

${ }^{1}$ Topigs Norsvin Research Center B.V., P.O. Box 43, 6640 AA Beuningen, The Netherlands; ${ }^{2}$ Wageningen University and Research, Wageningen Livestock Research, P.O. Box 338, 6700 AH Wageningen, The Netherlands; ${ }^{3}$ PEGASE, INRAE, Institut Agro, 35590, Saint-Gilles, France; ${ }^{4}$ Schothorst Feed Research B.V., P.O. Box 533, 8200 AM Lelystad, The Netherlands; ${ }^{5}$ Wageningen University and Research, Animal Breeding and Genomics, P.O. Box 338, 6700 AH Wageningen, The Netherlands

Accepted (Animal) 


\section{Abstract}

Providing pigs a diet that matches their nutrient requirements involves optimizing the diet based on the nutrient digestibility values of the considered feed ingredients. Feeding the same quantity of a diet to pigs with similar BW but with different requirements, however, can result in a different average daily gain (ADG) and backfat thickness (BF) between pigs. Digestibility may contribute to this variation in efficiency. We investigated variation in feed efficiency traits in grower-finisher pigs associated with variation in faecal digestibility values, independent of feed intake at the time of measuring faecal digestibility. Considered traits were ADG, average daily feed intake (ADFI), feed conversion ratio (FCR), BF and residual feed intake (RFI). Feed intake, BW, and BF data of 160 three-way crossbreed grower-finisher pigs ( 80 female and 80 male) were collected during two phases, from day 0 of the experiment (mean BW $23 \mathrm{~kg}$ ) till day 56 (mean BW $70 \mathrm{~kg}$ ) and from day 56 to slaughter (mean BW $121 \mathrm{~kg}$ ). Pigs were either fed a diet based on corn/soybean meal or a more fibrous diet based on wheat/barley/by-products, with titanium dioxide as indigestible marker. Faecal samples of 105 pigs were collected on the day before slaughter and used to determine apparent faecal digestibility of DM, ash, organic matter (OM), CP, crude fat (CFat), crude fibre (CF), and to calculate the digestibility of non-starch polysaccharides (NSP) and energy (E). The effects of diet, sex and covariate feed intake at sampling (Fls) on faecal digestibility values were estimated and were significant for all except for CFat. Faecal digestibility values of each individual pig determined at the day before slaughter, corrected for diet, sex and Fls, were used to estimate their association with ADG, ADFI, FCR, BF, and RFI. In the first phase, a one percent unit increase in faecal digestibility of $D M$, ash, OM, E, CP, CFat, CF, NSP, and Ash individually was related to 0.01 to 0.03 units reduction in FCR and 6 to $23 \mathrm{~g} /$ day reduction in RFI. A unit increase in CP digestibility was related to 0.1 $\mathrm{mm}$ increase in $\mathrm{BF}$ and $10 \mathrm{~g} /$ day increase in $\mathrm{ADG}$. In the second phase, a one percent unit increase in faecal digestibility of DM, CP and Ash was related to a decrease of 16 to $20 \mathrm{~g} /$ day in RFI. In conclusion, the relationship between variation in feed efficiency traits and faecal digestibility values is different across the developmental stages of a pig.

Keywords: Feed efficiency, Digestion, Feed intake, Genetics, Swine 


\subsection{Implications}

This study shows the importance of measuring feed efficiency traits at several timepoints in the life of a grower-finisher pig, especially when differences in nutrient digestion underlying variation in feed efficiency traits are studied. Differences in faecal nutrient digestibility between sexes can be used to further support the conceptual development of precision feeding in pig production, especially in systems where boars or barrows and gilts are housed separately.

\subsection{Introduction}

Both nutritionists and geneticists aim to increase feed efficiency in grower-finisher pigs, as feed comprises the main cost of production. In swine nutrition, increased feed efficiency is achieved by formulating diets that are balanced in terms of nutrient supply relative to the nutrient requirements of the animal, using different dietary ingredients and considering their nutrient composition and digestibility at ileal or faecal level. Although diet ingredient composition is the main source of variation in nutrient digestibility between pigs, there is also variation in the capability to digest nutrients between individual pigs that are fed the same diet (Ouweltjes et al., 2018). Variation among pigs for a wide range of performance traits is the main focus of study in pig genetics. In selection experiments, several generations of divergent selection for feed efficiency resulted in lines of pigs showing not only differences in feed efficiency, but also sometimes in differences in faecal digestibility of nutrients (Harris et al., 2012; Mauch et al., 2018). The increase in feed efficiency was mainly the result of a decrease in feed intake, while body weight gain was similar between the genetic lines. A lower feed intake, either restricted or voluntary, results in increased faecal digestibility values (Cunningham et al., 1962; De Haer and De Vries, 1993). Even after correction for the level of feed intake, differences in digestibility of dietary energy were noticed between feed efficiency lines (Harris et al., 2012). Still, the difference in faecal digestibility of DM and nitrogen between the selection lines disappeared when correcting for differences in voluntary feed intake between lines. Therefore, the question remains whether an increased digestive capacity of pigs leads to an increased feed efficiency.

The aim of this study was to investigate the variation in feed efficiency traits of grower-finishers pigs associated with the variation in faecal digestibility values, independent of variation in feed intake. Considered traits were average daily gain $(A D G)$, average daily feed intake (ADFI), feed conversion ratio (FCR), backfat thickness (BF) and residual feed intake (RFI). 


\subsection{Material and methods}

\subsubsection{Animals and experimental design}

Pigs used in this study were part of a larger experiment described by Godinho et al. (2018) and originated from a three-way cross, i.e. Synthetic boar x (Large White $x$ Landrace) sow. Part of the data used in the present study has been described previously and was used to predict faecal digestibility values based on faecal microbiota composition (Verschuren et al., 2020). Phenotypic data were available for 160 pigs, 80 intact boars and 80 gilts, originating from 21 litters. Due to death (6 animals) and insufficient faecal sample volume (49 animals) data of 105 pigs were used for the present study. All pigs were kept under commercial conditions at the experimental facilities of Schothorst Feed Research B.V. (Lelystad, The Netherlands). Before the start of the study pigs were housed per litter, with approximately $20 \%$ of the pigs being cross-fostered. All pigs were fed the same diet in this period. The pigs entered the study at 59 to 67 days of age (day 0 of the experiment) in two groups and the groups entered the study 20 days apart. Ten pigs were housed per pen and each pig was allowed a minimal space of $1 \mathrm{~m}^{2}$. Floors of the pens were $60 \%$ concrete and $40 \%$ slatted. There were eight pens per room and one room was used per entrance date. Littermates were randomly distributed over the two diets and males and females were housed in separate pens. Erroneously, due to the random distribution of littermates there was one litter that was completely allocated to a single pen. The pigs were kept in the facilities until they reached a live weight at slaughter of approximately $120 \mathrm{~kg}$ (mean age 167 days).

\subsubsection{Feeding strategy}

Two diets were used in the study, a diet based on corn/soybean meal (CS) as typically fed to commercial grower-finisher pigs in The Americas and a more fibrous diet based on wheat/barley/by-products (WB) as typically fed in Europe (Table 2.1). The pigs were fed ad libitum throughout the study, where 17 boars and 32 gilts were fed the CS diet, and 24 boars and 33 gilts were fed the WB diet. The pigs were fed a starter diet from day 0 to day 25 , a grower diet from day 25 to day 67 , and a finisher diet from day 67 until they reached slaughter weight. The diets were formulated on a fixed ratio of standardized ileal digestible lysine to net energy. The standardized ileal digestible lysine to net energy ratio changed over time, being $1.12 \mathrm{~g} / \mathrm{MJ}$ in the starter diet, $0.94 \mathrm{~g} / \mathrm{MJ}$ in the grower diet and $0.73 \mathrm{~g} / \mathrm{MJ}$ in the finisher diet. The decrease of standardized ileal digestible lysine to net energy ratio in grower and finisher diets was mainly achieved by exchanging soybean meal with corn for the CS diet, and peas with wheat for the WB diets. An additional premix was added to the 
finisher diets containing titanium dioxide as inert marker $(2.5 \mathrm{~g} / \mathrm{kg}$ at the level of the diet). The experimental diets were pelleted and produced by ABZ Diervoeding, Leusden, The Netherlands.

Table 2.1 Ingredient and calculated composition of the diets fed to the grower-finisher pigs, as-fed basis

\begin{tabular}{|c|c|c|c|c|c|c|}
\hline \multirow[b]{2}{*}{ Item } & \multicolumn{2}{|c|}{$\begin{array}{c}\text { Starter } \\
\text { (day } 0 \text { to } 25 \text { ) } \\
\end{array}$} & \multicolumn{2}{|c|}{$\begin{array}{c}\text { Grower } \\
\text { (day } 25 \text { to } 67 \text { ) }\end{array}$} & \multicolumn{2}{|c|}{$\begin{array}{c}\text { Finisher }^{1} \\
\text { (day } 67 \text { to end) }\end{array}$} \\
\hline & CS & WB & CS & WB & CS & WB \\
\hline \multicolumn{7}{|l|}{ Ingredient, $\mathrm{g} / \mathrm{kg}$} \\
\hline Corn & 647.1 & - & 698.4 & - & 755.1 & - \\
\hline Corn gluten feed & 18.1 & - & 25.0 & 50.0 & 50.0 & 50.0 \\
\hline $\begin{array}{l}\text { Soybean meal }(48 \% \\
\text { CP) }\end{array}$ & 240.5 & 100.0 & 180.5 & 21.5 & 98.3 & - \\
\hline Soybean hulls & - & - & - & 14.3 & - & 50.0 \\
\hline Soybean oil & - & 25.0 & - & 0.3 & - & - \\
\hline Barley & - & 200.0 & - & 100.0 & - & 150.0 \\
\hline Wheat & - & 321.9 & - & 400.0 & - & 350.0 \\
\hline Wheat middlings & - & - & - & 50.0 & - & 125.0 \\
\hline Rapeseed meal & - & 63.0 & - & 80.0 & - & 100.0 \\
\hline Sunflower seed meal & - & 80.0 & - & 80.0 & - & 21.9 \\
\hline Palmkernel meal & - & - & - & 50.0 & - & 50.0 \\
\hline Palm oil & 5.0 & 17.3 & 5.0 & 16.0 & 5.0 & 5.0 \\
\hline Field peas & - & 120.0 & - & 29.4 & - & - \\
\hline Sugarcane molasses & 40.0 & 30.0 & 50.0 & 50.0 & 50.0 & 50.0 \\
\hline Poultry fat & - & - & - & 27.5 & - & 29.4 \\
\hline $\begin{array}{l}\text { Monocalcium } \\
\text { phosphate }\end{array}$ & 6.7 & 5.3 & 2.0 & - & 0.7 & - \\
\hline Salt & 2.7 & 2.1 & 2.4 & 1.8 & 1.8 & 2.1 \\
\hline Sodium bicarbonate & - & 1.1 & 1.0 & 1.0 & 3.4 & - \\
\hline Phytase $^{2}$ & 5.0 & 5.0 & 5.0 & 5.0 & 5.0 & 1.9 \\
\hline Limestone & 11.6 & 10.9 & 9.4 & 8.9 & 9.9 & 4.0 \\
\hline AA premix ${ }^{3}$ & 17.3 & 12.5 & 17.3 & 10.2 & 16.7 & 6.7 \\
\hline Lys + Trp premix & 7.8 & 4.3 & 8.3 & 3.6 & 9.2 & - \\
\hline Lys HCl (L 79\%) & 2.4 & 3.8 & 2.2 & 4.3 & 1.9 & 3.3 \\
\hline Met (DL 99\%) & 1.6 & 1.3 & 1.4 & 0.7 & 0.8 & 0.1 \\
\hline $\operatorname{Thr}(\mathrm{L} 98 \%)$ & 1.5 & 1.7 & 1.5 & 1.6 & 1.5 & 0.9 \\
\hline Val (L 10\%) & - & 1.4 & - & - & - & - \\
\hline Vitamin premix ${ }^{4}$ & 1.0 & 1.0 & & & & \\
\hline $\begin{array}{l}\text { Vitamin-trace mineral } \\
\text { premix } 1^{5}\end{array}$ & 1.0 & 1.0 & & & & \\
\hline $\begin{array}{l}\text { Vitamin-trace mineral } \\
\text { premix } 2^{6}\end{array}$ & 4.0 & 4.0 & 4.0 & 4.0 & 4.0 & 4.0 \\
\hline
\end{tabular}




\section{Feed efficiency and nutrient digestibility in pigs}

\begin{tabular}{lcccccc}
$\begin{array}{l}\text { Nutrient composition, } \\
\text { g/kg }\end{array}$ & & & & & \\
NE, MJ/kg & 9.9 & 9.9 & 10.1 & 9.7 & 10.3 & 9.3 \\
Moisture & 127 & 126 & 130 & 126 & $122(130)^{*}$ & $110(129)^{*}$ \\
Ash & 51 & 52 & 42 & 47 & $38(38)^{*}$ & $43(42)^{*}$ \\
Crude protein & 182 & 190 & 159 & 166 & $122(128)^{*}$ & $140(147)^{*}$ \\
Crude fat & 34 & 58 & 35 & 64 & $39(36)^{*}$ & $61(57)^{*}$ \\
Crude fibre & 24 & 45 & 24 & 60 & $29(25)^{*}$ & $82(71)^{*}$ \\
Starch & 437 & 360 & 471 & 335 & $493(512)^{*}$ & $323(334)^{*}$ \\
Sugar & 44 & 50 & 46 & 58 & 42 & 59 \\
NSP & 135 & 170 & 130 & 216 & 126 & 246 \\
Ca & 6.9 & 6.9 & 5.2 & 5.5 & 5.0 & 3.8 \\
P & 4.8 & 5.5 & 3.6 & 4.7 & 3.2 & 4.7 \\
SID Lys & 11.1 & 11.1 & 9.5 & 9.1 & 7.5 & 6.8 \\
SID Met + Cys & 6.6 & 6.6 & 5.9 & 5.6 & 4.6 & 4.6 \\
SID Thr & 7.1 & 7.1 & 6.3 & 6.0 & 5.2 & 4.7 \\
SID Trp & 2.1 & 2.1 & 1.8 & 1.7 & 1.4 & 1.3 \\
\hline
\end{tabular}

$\mathrm{CS}=$ a diet based on corn/soybean meal; $\mathrm{WB}=$ a diet based on wheat/barley/by-products; $\mathrm{AA}$ = amino acid; Lys = Lysine; $\operatorname{Trp}=$ Tryptophan; Met = Methionine; Thr = Threonine, Val = Valine; $\mathrm{NE}=$ Net energy, NSP = Non-starch polysaccharides; SID = Standardized ileal digestible; Cys = Cystine.

${ }^{1}$ An additional premix was added to the finisher diets containing titanium dioxide as inert marker $(2.5 \mathrm{~g} / \mathrm{kg}$ at the level of the diet)

${ }^{2}$ Phyzyme XP (Dupont, Wilmington, Delaware, United states of America)/ Assumed P released 500 FTU: 1.12 g digestible $P / \mathrm{kg}$.

${ }^{3}$ Mixture of free Lys, Met, Thr, Trp, and Val to equalize dietary levels of SID amino acids relative to the net energy value of the diet. ${ }^{4}$ Supplied per kilogram of feed: $2500 \mathrm{IU}$ of vitamin A, 500 IU of vitamin D3, and 5 IU of vitamin E (Mervit AD3E; PreMervo, Utrecht, the Netherlands).

${ }^{5}$ Supplied per kilogram of feed: $12 \mathrm{mg}$ of Fe (ferrous sulphate), $10 \mathrm{mg}$ of $\mathrm{Mn}$ (manganous oxide), $0.04 \mathrm{mg}$ of Co cobalt oxide), $0.12 \mathrm{~g}$ of $\mathrm{Ca}, 0.0501 \mathrm{~g}$ of $\mathrm{P}, 0.04 \mathrm{mg}$ of I (potassium iodide), $1000 \mathrm{IU}$ of vitamin A, $100 \mathrm{IU}$ of vitamin D3, $5 \mathrm{IU}$ of vitamin E, $0.4 \mathrm{mg}$ of vitamin B1, $0.8 \mathrm{mg}$ of vitamin B2, $2 \mathrm{mg}$ of pantothenic acid, $4 \mathrm{mg}$ of niacin, $0.4 \mathrm{mg}$ of vitamin B6, $0.2 \mathrm{mg}$ of folate, $0.003 \mathrm{mg}$ of vitamin B12, $10 \mathrm{mg}$ of vitamin C, $0.01 \mathrm{mg}$ of biotin, $0.2 \mathrm{mg}$ of vitamin K3, and 40 $\mathrm{mg}$ of choline (Mervit Sporavit; PreMervo, Utrecht, the Netherlands).

${ }^{6}$ Supplied per kilogram of premix: $0.4 \mathrm{~g}$ of $\mathrm{Ca}, 15 \mathrm{mg}$ of $\mathrm{Cu}$ (copper sulphate), $80 \mathrm{mg}$ of $\mathrm{Fe}$ (ferrous sulphate), $24 \mathrm{mg}$ of $\mathrm{Mn}$ (manganous oxide), $62 \mathrm{mg}$ of $\mathrm{Zn}$ (zinc oxide), $0.04 \mathrm{mg}$ of Co (cobalt oxide), $0.4 \mathrm{mg}$ of I (potassium iodide), $0.2 \mathrm{mg}$ of Se (sodium selenite), $7500 \mathrm{IU}$ of vitamin A, 1500 IU of vitamin D3, 25 IU of vitamin E, $4 \mathrm{mg}$ of vitamin B2, $6 \mathrm{mg}$ of pantothenate, $30 \mathrm{mg}$ of niacin, $0.02 \mathrm{mg}$ of vitamin B12, and $0.752 \mathrm{mg}$ of vitamin K3 (Mervit START M220; PreMervo, Utrecht, the Netherlands).

${ }^{7}$ Based on chemical composition, digestibility, and energy values for pigs from the Centraal Veevoeder Bureau Livestock Feed Table (Centraal Veevoeder Bureau, 2019).

*Based on wet chemistry analysis, with values based on chemical composition within brackets. 


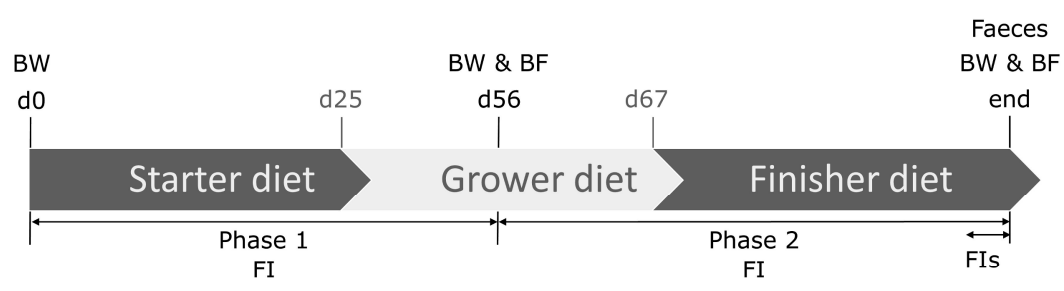

Figure 2.1 Experimental design and measurements according to the experimental phase and diet fed to the grower-finisher pigs. At onset of the experiment (d0) body weight (BW) was recorded and individual feed intake registration (FI) started. One day before slaughter (end) BW and backfat thickness (BF) were recorded and faecal samples were collected. Feed intake at sampling (FIs) was calculated as feed intake over the last 3 days of the experiment.

\subsubsection{Measurements and sampling}

The experimental facilities were equipped with IVOG feeding stations (INSENTEC, Marknesse, The Netherlands) that register individual feed intake of group housed pigs. All pigs had ear tags with unique numbering, therefore, individual feed intake records were available for all pigs for each day on test. Pigs were weighted at day 0 (mean age 64 days), day 56 (mean age 120 days) and at the end of the study (104 \pm 6.7 days in the experiment, mean age 167 days), dividing the experimental period in two phases, the first one being from day 0 till day 56 of the experiment, and the second one being from day 56 till the end of the experiment (Figure 2.1). The BF measurements were recorded at day 56 of the trial and at the end of the trial using an ultrasound device (Renco Lean Meater; Renco Corp., Minneapolis, USA). The ADFI was calculated as the cumulative individual daily feed intake records divided by the timespan over which the feed intake records were recorded. In addition, the feed intake at sampling (FIs) was calculated as the ADFI over the final three days of the experiment, excluding the day of faecal sample collection due to prevent effects of animal handling during faeces collection on feed intake. The ADG was calculated as the difference between BW measurements divided by the timespan between the measurements, whereas the FCR was calculated as the ratio between ADFI and ADG. The RFI was obtained for each phase separately as the residual term of the regression (Cai et al., 2008):

$A D F I=\mu+b_{1} B W_{o n}+b_{2} B W_{o f f}+b_{3} B F+b_{4} A D G+b_{5} O_{\text {age }}+e$ in which ADFI, BF, and ADG are described previously, $\mu$ is the mean ADFI of the pigs, $B W_{\text {on }}$ is the $\mathrm{BW}$ at the start of either phase, $B W_{\text {off }}$ is the $\mathrm{BW}$ at the end of either phase, $O_{\text {age }}$ is the age at the start of either phase, $b_{1}, b_{2}, b_{3}, b_{4}$, and $b_{5}$ are the linear 


\section{Feed efficiency and nutrient digestibility in pigs}

coefficients of the regression on covariates, and $e$ is the RFI. The mixed procedure (SAS 9.3; SAS Inst. Inc., Cary, NC) was used to obtain the RFI.

One day before slaughter, individual faecal grab samples were collected, stored at 4 ${ }^{\circ} \mathrm{C}$, freeze-dried and milled over a $1 \mathrm{~mm}$ sieve prior to chemical analyses. Faecal samples were analysed from 105 out of 160 pigs, related to death of a few pigs (6 animals) or availability of insufficient sample material (49 animal). Diets and faecal samples were analysed in duplicate for moisture, ash, CP, crude fat (CFat), crude fibre (CF), and titanium oxide as marker using the following methods respectively: ISO 6496, NEN 3329, ISO/CD 15670, ISO/FDIS 6492 method B, ISO-6865:2001, and EEG 26-11-1992 nr.L344/35-37 (Method based on Short et al. (1996)). Diets were also analysed in duplicate for starch (NEN-ISO 15914:2005 en), whereas, based on the faecal starch digestibility of the main dietary ingredients (Centraal Veevoeder Bureau, 2019), no starch was assumed to be present in faeces.

The concentrations of DM, organic matter (OM), non-starch polysaccharides (NSP) and energy (E) of the diets and faeces samples were calculated as indicated below.

$\mathrm{DM}(\mathrm{g} / \mathrm{kg})$ was calculated as:

1000 - moisture $(\mathrm{g} / \mathrm{kg})$

OM $(\mathrm{g} / \mathrm{kg})$ was calculated as:

$D M(g / k g)-A s h(g / k g)$,

the NSP fraction $(\mathrm{g} / \mathrm{kg})$ was calculated as:

$D M(g / k g)-((A s h(g / k g)+C P(g / k g)+C F a t(g / k g)+\operatorname{starch}(g /$ $\mathrm{kg})$ ),

and the $\mathbf{E}(\mathrm{kJ} / \mathrm{kg} \mathrm{DM})$ was calculated as (Centraal Veevoeder Bureau, 2019):

$24.14 \times C P(g / k g)+36.57 \times \operatorname{CFat}(\mathrm{g} / \mathrm{kg})+20.92 \times C F(g / k g)+16.99 \times$ nitrogen - free extract $(g / k g)$,

with nitrogen-free extract $(\mathrm{g} / \mathrm{kg})$ calculated as:

$D M(g / k g)-((A s h(g / k g)+C F a t(g / k g)+C P(g / k g)+C F(g / k g))$.

Apparent faecal digestibility values, also known as apparent total tract digestibility values, were calculated in percentages based on concentrations of the marker and the nutrient in the diet and faeces as:

$\left[1-\left(\frac{\text { conc.of marker in the diet }\left(\frac{\mathrm{mg}}{\mathrm{kg}} D M\right)}{\text { conc.of marker in the faeces }\left(\frac{\mathrm{mg}}{\mathrm{kg}} D M\right)}\right)\left(\frac{\text { conc.of nutrient in the faeces }\left(\frac{\mathrm{g}}{\mathrm{kg}} D M\right)}{\text { conc.of nutrient in the } \operatorname{diet}\left(\frac{\mathrm{g}}{\mathrm{kg}} D M\right)}\right)\right] \times$ $100 \%$ 


\subsubsection{Statistical analysis}

The experimental set-up followed a split-plot design in a $2 \times 2$ factorial arrangement, the two factors being diet (CS vs. WB diets) and sex (intact boars vs. gilts). Individual pigs were considered the experimental unit. First, we investigated the difference in ADG, ADFI, FCR, BF and RFI between the two diets and sexes, and obtained their residual variation after correction for these factors (model 1). Second, we investigated the relationship of faecal digestibility values with diet, sex and feed intake, and obtained their residual variation after correction for these factors (model 2). Third, we investigated to which extent variation in feed efficiency traits across individual pigs was related to variation in their faecal digestibility values, corrected for diet, sex and feed intake (model 3). The three statistical models were:

$X_{i j k l m}=\mu+\operatorname{sex}_{i}+$ diet $_{j}+\operatorname{sex}_{i} \cdot$ diet $_{j}+$ group $_{k}+b_{a} B W_{l}+$ pen $_{m}+e_{i j k l m}$ (1)

$Y_{i j k l m}=\mu+\operatorname{sex}_{i}+$ diet $_{j}+\operatorname{sex}_{i} \cdot$ diet $_{j}+\operatorname{group}_{k}+b_{b}$ FIs $_{(j l)}+$ pen $_{m}+$

$\varepsilon_{i j k l m}(2)$

$X_{i j k l m}=\mu+\operatorname{sex}_{i}+$ diet $_{j}+\operatorname{sex}_{i} \cdot$ diet $_{j}+\operatorname{group}_{k}+b_{a} B W_{l}+b_{c}$ CFD $_{l}+$

pen $_{m}+\epsilon_{i j k l m}$ (3)

where $X_{i j k l m}$ is observed ADG, ADFI, FCR, BF, or RFI from either phase of the experiment and $Y_{i j k l m}$ is observed faecal DM, OM, E, CP, CFat, CF, NSP or ash digestibility for each pig $I(I=1 \ldots 105)$ with known group ( $k=1$ or 2$)$, sex $i$ ( $i=$ gilt or boar) and diet $j$ ( $j=$ CS or WB diets). $\mu$ is the mean across pigs. $b_{a}$ is the regression coefficient for $B W_{l}$, which is birth weight for $A D G$, live $B W$ at start of either phase for $\mathrm{ADFI}, \mathrm{FCR}$ and $\mathrm{RFI}$, or live $\mathrm{BW}$ at moment of measuring for $\mathrm{BF} . b_{b}$ is the regression coefficient for Fls and has an interaction term between Fls and diet for faecal ash digestibility. $b_{c}$ is the regression coefficient for corrected faecal digestibility $\left(C F D_{l}\right)$ of each nutrient individually (DM, OM, E, CP, CFat, CF, NSP or ash), and CFD, is obtained as the random residual term $\varepsilon_{i j k l m}$ of model 2 . Thus, model 3 was applied eight times, i.e. once for each nutrient, for ADG, ADFI, FCR, BF, and RFI in either phase. $\mathrm{Pen}_{m}$ is the random effect of the $m^{\text {th }}$ housing pen assumed to be normally distributed $\sim N\left(0, \mathbf{I} \sigma_{\text {pen }}^{2}\right)$, and $e_{i j k l m}, \varepsilon_{i j k l m}$, and $\epsilon_{i j k l m}$ are random residual terms assumed to be normally distributed $\sim N\left(0, \mathbf{I} \sigma_{\mathrm{e}}^{2}\right)$. The mixed procedure (SAS 9.3; SAS Inst. Inc., Cary, $\mathrm{NC}$ ) was used to fit the models. Hereafter, variance will be used as the statistical term for variation. Total variance in $X_{i j k l m}$ and $Y_{i j k l m}$, i.e. $\operatorname{Var}\left(X_{i j k l m}\right)$ and $\operatorname{Var}\left(Y_{i j k l m}\right)$, were calculated and consecutively explained variance by the models was calculated as:

$$
\begin{aligned}
& \frac{\operatorname{Var}\left(X_{i j k l m}\right)-\operatorname{Var}\left(e_{i j k l m}\right)}{\operatorname{Var}\left(X_{i j k l m}\right)} \times 100 \%, \\
& \frac{\operatorname{Var}\left(Y_{i j k l m}\right)-\operatorname{Var}\left(\varepsilon_{i j k l m}\right)}{\operatorname{Var}\left(Y_{i j k l m}\right)} \times 100 \% .
\end{aligned}
$$




\section{Feed efficiency and nutrient digestibility in pigs}

Least squares means of model 1 and 2 were calculated per factor, and statistical differences between least squares means for diet, sex, and diet by sex combinations were calculated using a post hoc Tukey test. Any differences in least squares means and estimated coefficients of regression on $C F D_{l}$ (i.e. $\hat{b}_{c}$ ) were declared to be significant at $\mathrm{P}<0.05$ and $P$-values between 0.05 and 0.10 were considered indicative of a trend. Difference between diets or sexes in feed efficiency traits might partly be due to the differences in nutrient digestibility between diets or sexes, so only considering the difference in explained variance between models 1 and 3 could give biased estimates. Hence, variance in feed efficiency traits related to variance in corrected faecal digestibility values was calculated using the estimated regression coefficient from model 3 and total variance in feed efficiency traits as:

$\frac{\operatorname{Var}\left(\hat{b}_{c} \times C F D_{l}\right)}{\operatorname{Var}\left(X_{i j k l m}\right)} \times 100 \%$.

\subsection{Results}

\subsubsection{Feed efficiency traits}

Pigs on different diets and pigs from different sexes showed some differences in ADG, ADFI, FCR, BF and RFI during both phases and the overall phase (Table 2.2). Mean BW at start of the first phase was $22.6 \mathrm{~kg}$, at start of the second phase $70.2 \mathrm{~kg}$, and at the end of the experiment $121.1 \mathrm{~kg}$ (data not shown). The BW co-variates in model 1 were significant for all traits except for RFI. There was a tendency for boars having a lower BW than gilts at start of the first phase of the experiment $(P=0.097)$ and at the start of the second phase $(P=0.085)$ (data not shown). Boars had a lower FCR and RFI than gilts in the first phase ( $P=0.017$ and $P=0.009$, respectively) and in the second phase there was a tendency in the same direction for FCR but not for RFI ( $P=0.069$ and $P=0.232$, respectively). Gilts tended to have a higher ADFI than boars in the first phase of the experiment $(P=0.075)$, which resulted in a higher ADG for gilts fed the CS diet compared to boars fed that diet $(P=0.015)$. In contrast, there was no significant difference between the sexes in ADFI during the second phase ( $P$ $=0.771$ ), and boars fed the WB diet had a higher ADG than gilts fed the same diet ( $P$ $=0.02$ ). Whereas at the end of the first phase of the experiment the boars have a higher $\mathrm{BF}(P=0.036)$, at the end of the second phasegilts had the highest $\mathrm{BF}(P=$ $0.017)$. The BF of pigs fed the WB diet was lower than pigs fed the CS diet at the end of the second phase $(P<0.001)$, which was the only significant effect of diet on the feed efficiency traits. Over the total period the males had a lower FCR and RFI $(P=$ 0.018 and $P=0.049$, respectively). The explained variance by the models estimating 
the effects of diet, sex, the interaction between diet and sex, group and BW on feed efficiency traits ranged from $2.2 \%$ for RFI during the first phase to $52.6 \%$ for ADFI during the second phase.

Table 2.2 Performance of male and female grower-finisher pigs fed one out of two experimental diets ${ }^{1}$, and the explained variance by the models

\begin{tabular}{|c|c|c|c|c|c|c|c|c|c|}
\hline \multirow[b]{2}{*}{ Trait $^{2}$} & \multicolumn{2}{|c|}{$\mathrm{CS}^{3}$} & \multicolumn{2}{|c|}{$W^{3}$} & \multicolumn{3}{|c|}{ P-value } & \multicolumn{2}{|c|}{ Variance } \\
\hline & $B$ & $G$ & $B$ & G & Diet & Sex & D*S & RMSE & Exp \\
\hline \multicolumn{10}{|l|}{ Phase 1} \\
\hline ADFI, g/day & 1564 & 1713 & 1640 & 1676 & 0.695 & 0.075 & 0.266 & 212 & 43.1 \\
\hline ADG, g/day & $761^{a}$ & $871^{b}$ & $849^{a b}$ & $838^{\mathrm{ab}}$ & 0.361 & 0.102 & 0.046 & 101 & 20.9 \\
\hline FCR, g/g & 1.93 & 2.01 & 1.92 & 2.03 & 0.822 & 0.017 & 0.752 & 0.17 & 19.8 \\
\hline $\mathrm{BF}, \mathrm{mm}$ & 7.4 & 7.0 & 7.1 & 6.8 & 0.073 & 0.036 & 0.796 & 0.8 & 28.1 \\
\hline RFI, g/day & -48.0 & 27.8 & -51.9 & 34.9 & 0.957 & 0.009 & 0.859 & 143 & 2.2 \\
\hline \multicolumn{10}{|l|}{ Phase 2} \\
\hline ADFI, g/day & 2873 & 3044 & 2943 & 2832 & 0.478 & 0.771 & 0.170 & 296 & 52.6 \\
\hline ADG, g/day & $1066^{a b}$ & $1147^{a}$ & $1127^{a}$ & $1031^{b}$ & 0.375 & 0.803 & 0.006 & 139 & 16.0 \\
\hline FCR, g/g & 2.60 & 2.68 & 2.66 & 2.77 & 0.181 & 0.069 & 0.754 & 0.21 & 31.5 \\
\hline $\mathrm{BF}, \mathrm{mm}$ & 10.6 & 11.8 & 9.8 & 10.2 & $<0.001$ & 0.017 & 0.173 & 1.5 & 37.5 \\
\hline $\mathrm{RFI}, \mathrm{g} /$ day & -73.8 & -5.8 & 1.2 & 54.3 & 0.179 & 0.232 & 0.882 & 162 & 18.2 \\
\hline \multicolumn{10}{|l|}{ Total } \\
\hline ADFI, g/day & 2164 & 2299 & 2237 & 2198 & 0.788 & 0.357 & 0.089 & 225 & 40.0 \\
\hline ADG, g/day & $912^{\mathrm{a}}$ & $991^{b}$ & $976^{b}$ & $927^{a}$ & 0.980 & 0.426 & 0.001 & 93 & 15.9 \\
\hline FCR, g/g & 2.28 & 2.35 & 2.29 & 2.40 & 0.449 & 0.018 & 0.585 & 0.1 & 33.8 \\
\hline RFI, g/day & -52.9 & -7.1 & -21.2 & 51.0 & 0.128 & 0.049 & 0.640 & 125 & 5.8 \\
\hline
\end{tabular}

$\mathrm{CS}=$ a diet based on corn/soybean meal; $\mathrm{WB}=$ a diet based on wheat/barley/by-products; $\mathrm{B}=$ boars; $G$ = gilts; $D^{*} S=$ interaction between diet and sex effects; $\operatorname{Exp}=$ explained variance (\%) by the statistical model;

${ }^{1}$ Statistical model 1 including the effect of diet, sex, the interaction between diet and sex, group, body weight (birth weight for ADG, live body weight at start of each phase for ADFI, $\mathrm{FCR}$ and $\mathrm{RFI}$, or live body weight at moment of measuring for $\mathrm{BF}$ ), and pen;

2 Phase $1=$ from d0 till d56 of the experiment; Phase 2 = from d56 till the end of the experiment; Total = from d0 till the end of the experiment; $A D F I=$ Average daily feed intake; $A D G=$ Average daily gain; $F C R=$ Feed conversion ratio; $B F=$ Backfat thickness; $\mathrm{RFI}=$ Residual feed intake;

${ }^{3}$ Values are least squares means;

a,b Values within a row with different superscripts differ significantly at $P<0.05$.

\subsubsection{Apparent faecal digestibility}

Diet, sex, and Fls showed a clear effect on faecal digestibility values (Table 2.3). The faecal digestibility values were lower for pigs fed the WB diet compared to pigs fed the CS diet. Boars fed the WB diet had lower faecal digestibility values compared to gilts, except for CFat and ash, which were not different between sexes. All faecal digestibility values decreased with increasing Fls, except for CFat, which was not affected by Fls. There was no interaction between Fls and sex, and only a significant 


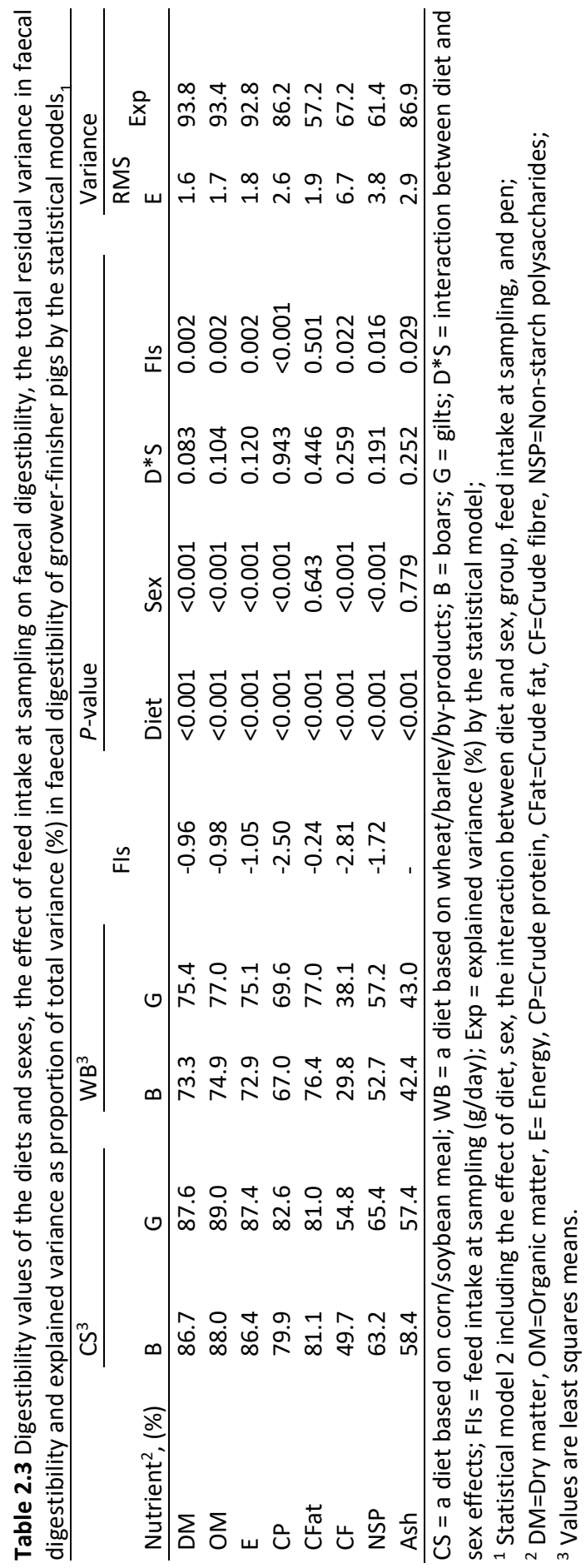


interaction between Fls and diet for faecal ash digestibility. The models estimating the effect of diet, sex, diet by sex interaction, group and FIs on faecal digestibility explained $57.2 \%$ of the variance in faecal CFat digestibility to $93.8 \%$ of the variance in faecal DM digestibility.

\subsubsection{Feed efficiency traits and faecal digestibility}

Faecal digestibility values were corrected for group, Fls, sex, diet and the interaction between sex and diet using model 2, and after this correction tested for its relationship with feed efficiency traits using model 3 (Table 2.4). Results comparing model 1 to model 3 are provided in supplementary Table 2.S1, and paint a similar picture as the results in Table 2.4. In the first phase of the experiment 3.7 to $7.6 \%$ of the variance in FCR and 4.3 to $7.0 \%$ of the variance in RFI was associated with all corrected faecal digestibility values. An increase in faecal digestibility values was related to a decrease in FCR and RFI in the first phase of the experiment, with the strongest relationship for DM: per percent unit of increased faecal DM digestibility a decrease of 0.03 units in FCR and $23 \mathrm{~g} /$ day in RFI (Table 2.5). Variance in BF at the end of the first phase was associated with corrected CP digestibility, with a unit increase in faecal digestibility of $\mathrm{CP}$ relating to an increase of $0.1 \mathrm{~mm}$ of BF. During the first phase of the experiment, 5.5\% of the variance in ADG was associated with $\mathrm{CP}$ digestibility, and a unit increase in $\mathrm{CP}$ digestibility was related to an increase of $10 \mathrm{~g} /$ day in ADG. There was no relationship between corrected faecal digestibility values and ADFI during the first phase of the experiment.

In the second phase of the experiment there was a significant effect for $1.9 \%$ of the variance in ADFI being associated with corrected faecal digestibility of CF, and tendencies for $\mathrm{DM}, \mathrm{OM}, \mathrm{E}$, and $\mathrm{CP}$, with a one percent unit increase in digestibility being related to a decrease of up to $35 \mathrm{~g} /$ day in ADFI. Up to $8.3 \%$ of the variance in RFI was associated with corrected DM, CP and ash digestibility, and tendencies for OM, E, CFat, and CF digestibility, for which an increase in faecal digestibility values was related to a decrease of up to $20 \mathrm{~g} /$ day in RFI. There was no significant relationship between any tested corrected faecal digestibility values and ADG, FCR or BF during the second phase of the experiment.

Over the total period there was no effect of any of the tested corrected faecal digestibility values and ADFI, ADG or FCR. There were, however, significant effects for all tested corrected faecal digestibility values with RFI, with an increase in digestibility being related to a decrease of up to $17 \mathrm{~g} /$ day in RFI. Of all corrected faecal digestibility relationships tested, CP was related to the highest percentage of 


\section{Feed efficiency and nutrient digestibility in pigs}

variance in the feed efficiency traits in both phases, whereas corrected faecal DM digestibility had the strongest relationship with the traits.

Table 2.4 Variance (\%) in performance traits of grower-finisher pigs explained by corrected faecal digestibility values ${ }^{1}$, with significant values depicted as is $(P<0.05)$, trends indicated as values with an asterisk $(P<0.1$ and $P>0.05)$ and non-significant associations as values in brackets $(P>0.1)^{2}$

\begin{tabular}{|c|c|c|c|c|c|c|c|c|}
\hline Trait $^{3}$ & DM & OM & $E$ & $\mathrm{CP}$ & CFat & CF & NSP & Ash \\
\hline \multicolumn{9}{|l|}{ Phase 1} \\
\hline ADFI, g/day & $(0.5)$ & $(0.4)$ & $(0.5)$ & $(0.1)$ & (1.3) & $1.6^{*}$ & $(0.5)$ & $(0.8)$ \\
\hline ADG, g/day & $2.7^{*}$ & $3.0^{*}$ & $2.8^{*}$ & 5.5 & $(0.7)$ & $(0.8)$ & (1.1) & $(0.0)$ \\
\hline FCR, g/g & 6.8 & 6.4 & 6.3 & 7.6 & 6.6 & 6.3 & 4.2 & 3.7 \\
\hline $\mathrm{BF}, \mathrm{mm}$ & $2.5^{*}$ & $2.3^{*}$ & $2.3^{*}$ & 2.9 & $(0.0)$ & (1.5) & (1.8) & $(0.7)$ \\
\hline RFI, g/day & 6.6 & 6.2 & 6.3 & 6.1 & 6.3 & 7.0 & 4.5 & 4.3 \\
\hline \multicolumn{9}{|l|}{ Phase 2} \\
\hline $\mathrm{ADFI}, \mathrm{g} / \mathrm{day}$ & $1.7^{*}$ & $1.6^{*}$ & $1.5^{*}$ & $1.7^{*}$ & $(0.5)$ & 1.9 & $1.4^{*}$ & $(0.8)$ \\
\hline$A D G, g / d a y$ & $(0.7)$ & $(0.7)$ & $(0.4)$ & $(0.1)$ & $(0.0)$ & $(2.2)$ & (1.)) & $(0.1)$ \\
\hline $\mathrm{FCR}, \mathrm{g} / \mathrm{g}$ & $(0.0)$ & $(0.0)$ & $(0.0)$ & (1.4) & (0.7) & $(0.2)$ & (0.3) & $2.4^{*}$ \\
\hline $\mathrm{BF}, \mathrm{mm}$ & $(0.2)$ & $(0.3)$ & $(0.4)$ & $(0.1)$ & $(0.2)$ & $(0.8)$ & $(0.4)$ & $(0.0)$ \\
\hline RFI, g/day & 3.3 & $2.7^{*}$ & $3.0^{*}$ & 8.3 & $2.5^{*}$ & $2.6^{*}$ & (1.7) & 6.4 \\
\hline \multicolumn{9}{|l|}{ Total } \\
\hline $\mathrm{ADFI}, \mathrm{g} / \mathrm{day}$ & $(0.6)$ & $(0.5)$ & $(0.5)$ & $(0.1)$ & $(0.5)$ & $1.7^{*}$ & $(0.7)$ & (1.0) \\
\hline$A D G, g / d a y$ & $(0.2)$ & $(0.3)$ & $(0.3)$ & $2.8^{*}$ & $(0.4)$ & $(0.2)$ & $(0.0)$ & $(0.0)$ \\
\hline FCR, g/g & (1.6) & (1.4) & (1.5) & 4.8 & 2.7 & (1.0) & $(0.4)$ & 3.4 \\
\hline RFI, g/day & 4.5 & 3.9 & 4.3 & 6.7 & 3.9 & 4.9 & $2.5^{*}$ & 6.1 \\
\hline \multicolumn{9}{|c|}{ 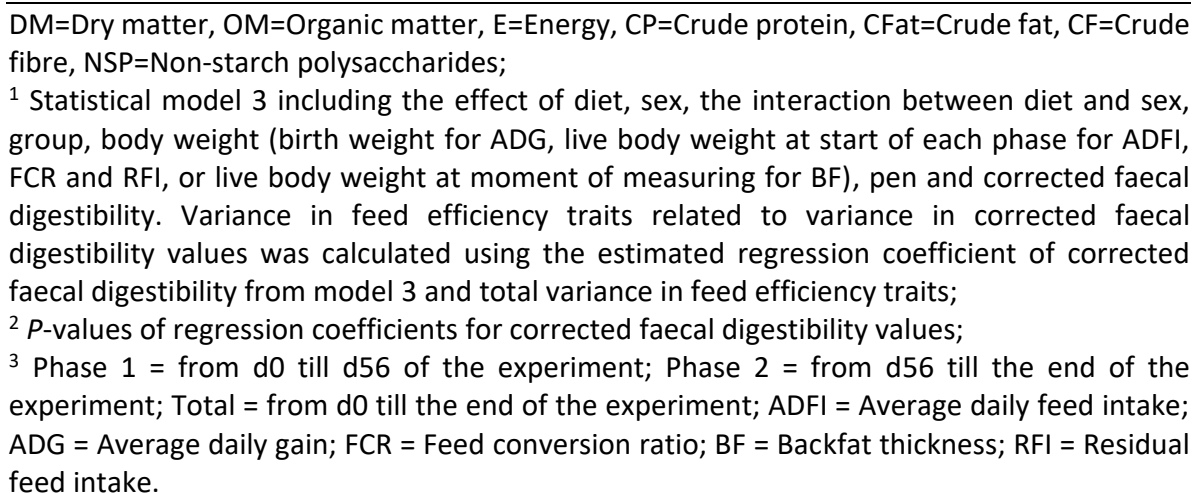 } \\
\hline
\end{tabular}




\subsection{Discussion}

\subsubsection{Variation in faecal digestibility}

In this study, we investigated the variation in ADG, ADFI, FCR, BF, and RFI in growerfinisher pigs associated with faecal digestibility of $D M, O M, E, C P, C F a t, C F$, and ash determined at the end of the fattening period. The variation in faecal digestibility values was for the largest part explained by diet. In literature, it is well established that dietary ingredients and nutrient composition affect faecal digestibility of nutrients (Le Goff and Noblet, 2001; Sauvant et al., 2004; Ouweltjes et al., 2018). Feed intake level affects faecal digestibility values as well (Cunningham et al., 1962; De Haer and De Vries, 1993). We estimated the effect of feed intake level on variation in faecal digestibility values and, even though the CS and WB diets had different calculated net energy values, an increase in Fls was associated with a reduction in faecal digestibility of all nutrients, except ash, to the same extent in both diets. The observed variation in faecal digestibility values between pigs fed the same diet could have been influenced by the method of collection of faeces samples (grab sampling on a single day), as it was shown that grab sampling of faeces over multiple days per animal provides more accurate estimates for nutrient digestibility values (Moughan et al., 1991; Agudelo et al., 2010). Between animal variation in faecal digestibility values, however, has been observed previously (Wilfart et al., 2007; Le Gall et al., 2009; Ouweltjes et al., 2018). Furthermore, Vigors et al. (2016b) observed that pigs with a low RFI do not only have a higher faecal DM, E, and CP digestibility compared to high RFI pigs fed the same diet, but also show an increased expression in intestinal tissue of the gene encoding for the enzyme sucrase-isomaltase, and for the genes SGLT1, FABP2, and GLUT2, which are related to intestinal nutrient transport. They also found a lower weight of the tissue of the total intestinal tract and a different faecal microbiota composition in pigs having a low compared to high RFI (Vigors et al., 2016b).These results suggest that between animal variation in faecal digestibility values may be related to differences in absorptive capacity of the intestinal tract, and to composition and activity of its residing microbiota in pigs.

Sex explained a large part of the variation in faecal digestibility values, with gilts showing higher digestibility values on both diets compared to intact males. The estimated higher digestibility of nutrients in gilts was not due to a lower feed intake in this sex, as feed intake was included as co-variable in the statistical model to explain variation in nutrient digestibility. Contradicting results on the effect of sex on nutrient digestibility in pigs have been reported in the literature. Noblet et al. (1993) found a higher faecal energy digestibility in gilts and no difference in nitrogen 
digestibility, whereas De Haer and De Vries (1993) observed no effect of sex on faecal energy digestibility and a higher faecal nitrogen digestibility in gilts when housed individually. Boars, however, had a higher faecal nitrogen digestibility than gilts when housed in groups (De Haer and De Vries, 1993). The higher faecal digestibility values of gilts might be due to differences in digestive capacity of the gut, as weaner gilts showed higher villus heights and higher small intestinal and pancreatic digestive enzyme activity compared to boars (Pluske et al., 2003). Effects of sex hormones might also play a role, since female steroid hormones are, amongst others, linked to intestinal hypomotility, inhibited gastric emptying, and increased bicarbonate production of the duodenal mucosa in humans (Freire et al., 2011). Differences in gut microbiota composition and fermentation activity, in particular in the hindgut, might explain part of the difference in faecal digestibility values between the sexes as well, because faecal microbiota composition is different between male and female pigs (Xiao et al., 2016; Verschuren et al., 2018). Although results in our study showed that the faecal digestibility of most nutrients was higher in gilts, FCR and RFI values were lower in boars. This indicates that differences in post-absorptive metabolism of nutrients in organs and tissues explain the difference between sexes in feed efficiency in grower-finisher pigs and even overcome the differences in faecal digestibility between sexes.

\subsubsection{Variation in feed efficiency traits related to faecal digestibility}

We found an association between feed efficiency traits and faecal digestibility ofDM, $\mathrm{OM}, \mathrm{E}, \mathrm{CP}, \mathrm{CFat}, \mathrm{CF}, \mathrm{NSP}$, and Ash. It might be that the faecal digestibility values, measured at the end of the grower-finisher period, are not fully representative for the whole grower-finisher period, as faecal digestibility of DM, OM, E, CP, CFat, NDF, hemicellulose, cellulose, lignin and NSP increases with age (Noblet et al., 2013; Le Sciellour et al., 2018; Ouweltjes et al., 2018). The increase in faecal digestibility values with age might differ between individual pigs. For instance, Le Sciellour et al. (2018) showed an interaction between breed and age on faecal digestibility of NDF, hemicellulose, and cellulose, but found no such effect on the faecal digestibility of OM, E, CP or lignin of pigs being 11 to 22 weeks of age. Also Noblet et al. (2013) found no interaction between sire effect and age on faecal digestibility of DM, OM, $\mathrm{E}$ and $\mathrm{CP}$ of pigs weighing 36 to $90 \mathrm{~kg}$. Therefore, our results on the relationship between the feed efficiency traits and faecal digestibility of DM, OM, E and CP are most likely representative for the entire grower-finisher period of the pig, whereas some caution should be taken when using and interpreting the relationship with faecal digestibility of CFat, CF, NSP and ash. 


\section{Feed efficiency and nutrient digestibility in pigs}

In the first phase of the experiment increases in ADG and BF, and decreases in FCR and RFI, were associated with an increase in corrected faecal digestibility values. In contrast, in the second phase only decreases in ADFI and RFI were associated with an increase in corrected faecal digestibility values. A possible explanation may lie in the regulation of feed intake in different growth stages. Young pigs ( $<50 \mathrm{~kg} \mathrm{BW})$ do not compensate for a decrease in dietary NE level by increasing feed intake when diets have a NE value below $10.5 \mathrm{MJ} / \mathrm{kg}$, whereas older pigs tend to maintain energy intake at a fixed level (Black et al., 1986; Fagundes et al., 2009; Quiniou and Noblet, 2012). Other factors, such as the physical capacity of the gut, are most likely limiting dietary feed intake in pigs that do not compensate for a lower NE value of the diet (Li and Patience, 2017). Also, when pigs are fed diets containing a fixed energy level, an increase in dietary protein level reduces feed intake (Henry et al., 1992; Le Bellego and Noblet, 2002), and an imbalanced amino acid profile reduces feed intake as well (Gloaguen et al., 2011; Gloaguen et al., 2012). Increased levels of the microbial fermentation product acetate in the blood can also reduce feed intake (Frost et al., 2014). Pigs showing a higher faecal nutrient digestibility have more dietary nutrients available for maintenance and growth compared to pigs with a lower faecal nutrient digestibility. However, if excess of a dietary nutrient or fermentation product is limiting feed intake of pigs, an increase in uptake of that nutrient or fermentation product would lead to a decreased feed intake. This provides a possible explanation for the reduced $\mathrm{ADFI}$ and $\mathrm{RFI}$ associated with an increased corrected faecal digestibility of $D M, O M, E, C P, C F$ and NSP during the second phase of our experiment. In contrast, during the first phase of the experiment other factors than dietary nutrient composition or fermentation products were most likely limiting feed intake of the pigs and, therefore, an increased availability of nutrients due to an increased nutrient digestibility resulted in a higher ADG and BF, and a lower FCR and RFI. Hence, the relationship between feed efficiency traits and nutrient digestibility most likely changes depending on nutrient composition of the diet and the developmental stage of the pig.

In this study, a higher faecal digestibility was related to a lower FCR in the first phase and a lower RFI in both phases of the experiment. This is in line with literature, as pigs with a high feed efficiency have been shown to either have the same or a higher faecal digestibility of DM, nitrogen, energy, and neutral detergent fibre compared to pigs with a low efficiency (Harris et al., 2012; Montagne et al., 2014; Vigors et al., 2016b; Mauch et al., 2018). Corrected faecal digestibility was related to up to $5.5 \%$ of the variation in $A D G, 7.6 \%$ of the variation in $F C R, 2.9 \%$ of the variation in $B F, 1.9 \%$ of the variation in $\mathrm{ADFI}$, and $8.3 \%$ of the variation in $\mathrm{RFI}$. In comparison, within 
population genetic variation of pigs explained up to $34 \%$ of the variation in ADG, $34 \%$ of the variation in $\mathrm{FCR}, 23 \%$ of the variation in lipid deposition, $42 \%$ of the variation in ADFI, and $29 \%$ of the variation in RFI in a study of which our pigs are a subsample (Godinho et al., 2018). Pigs with a different sire have different faecal digestibility of DM, OM, CP, and E (Noblet et al., 2013), suggesting that it might be possible to increase faecal digestibility by means of breeding. First results indicate that $\mathrm{OM}, \mathrm{E}$ and nitrogen digestibility are moderately to highly heritable and have a negative genetic correlation with FCR, ADFI, ADG, and RFI (Déru et al., 2021). These results are in line with our study, except for the unfavourable genetic correlation between faecal $\mathrm{N}$ digestibility and ADG, which is in contrast to our positive phenotypic correlation between faecal CP digestibility and ADG over the total grower-finisher period. The added value of measuring faecal digestibility on top of measuring ADG, ADFI and BF as selection parameter to genetically improve feed efficiency in pigs, therefore, is still uncertain. Since our dataset was too small to estimate such an added value, more research is needed to evaluate the possibility of improving feed efficiency of pigs by breeding for increased faecal digestibility.

\subsection{Conclusion}

There is substantial variation in faecal digestibility values between pigs fed a diet based on corn/soybean meal or wheat/barley/by-products. Part of the observed variation in feed efficiency traits was associated with variation in faecal digestibility values, but the results differed between young and older animals. Nevertheless, an increase in faecal digestibility values was related to a decrease in RFI over the entire grower-finisher period. In conclusion, the relationship between variation in feed efficiency traits and faecal digestibility values most likely differed between the developmental stages of a pig.

\subsection{Ethics approval}

This study was carried out in strict accordance with the recommendations in the European Guidelines for accommodation and care of animals. The protocol was approved by the Animal Care and Use Committee of Schothorst Feed Research, The Netherlands (Protocol Number: AVD 246002015120/132). 


\subsection{Data and model availability statement}

The data that support the findings of this study are available from the corresponding author upon reasonable request. None of the data were deposited in an official repository.

\subsection{Author ORCIDs}

L.M.G. Verschuren: https://orcid.org/0000-0001-5428-4174; D. Schokker: https://orcid.org/0000-0001-6082-7227; R. Bergsma: https://orcid.org/0000-00028254-5535; J. van Milgen: https://orcid.org/0000-0002-6131-5255; F. Molist: https://orcid.org/0000-0002-7915-3098; M.P.L. Calus: https://orcid.org/0000-00023213-704X; A.J.M. Jansman: https://orcid.org/0000-0002-5104-8261

\subsection{Author contributions}

L.M.G. Verschuren: Conceptualization, Formal Analysis, Methodology, Writing original draft, Writing - review \& editing. D. Schokker: Conceptualization, Data curation, Funding acquisition, Methodology, Writing - original draft, Writing - review \& editing. R. Bergsma: Data curation, Project administration, Writing - original draft, Writing -review \& editing. J. van Milgen: Formal analysis, Methodology, Supervision, Writing - review \& editing. F. Molist: Data curation, Project administration, Writing - original draft, writing -review \& editing. M.P.L. Calus: Conceptualization, Formal analysis, Methodology, Supervision, Writing - original draft, Writing - review \& editing. A.J.M. Jansman: Conceptualization, Methodology, Supervision, Writing original draft, Writing - review \& editing.

\subsection{Acknowledgements}

We like to thank Schothorst Feed Research and Topigs Norsvin for providing data.

\subsection{Financial support statement}

This study was financially supported by the Dutch Ministry of Economic Affairs (TKI Agri \& Food project 12039; Public-private partnership "Breed\&Feed4Food" code BO-22.04-010-001).

\subsection{Declaration of interest}

The authors declare no conflict of interest. 


\section{Fecal microbial composition associated with variation in feed efficiency in pigs depends on diet and sex}

L.M.G. Verschuren ${ }^{1,2}$, M.P.L. Calus ${ }^{2}$, A.J.M. Jansman², R. Bergsma ${ }^{1}$, E.F. Knol ${ }^{1}$, H. Gilbert ${ }^{3}$, O. Zemb ${ }^{3}$ ]

${ }^{1}$ Topigs Norsvin Research Center B.V., Beuningen, 6640 AA, The Netherlands;

${ }^{2}$ Wageningen UR, Livestock Research, Wageningen, $6700 \mathrm{AH}$, The Netherlands;

3 INRA - INPT - ENSAT - Université de Toulouse, GenPhySE, Castanet-Tolosan, 31326, France

Journal of Animal Science (2018) 96:1405-1418 


\begin{abstract}
Dietary fiber content and composition affect microbial composition and activity in the gut, which in turn influence energetic contribution of fermentation products to the metabolic energy supply in pigs. This may affect feed efficiency (FE) in pigs. The present study investigated the relationship between the fecal microbial composition and FE in individual growing-finishing pigs. In addition, the effects of diet composition and sex on the fecal microbiome were studied. Fecal samples were collected of 154 grower-finisher pigs (3-way crossbreeds) the day before slaughter. Pigs were either fed a diet based on corn/soybean meal (CS) or a diet based on wheat/barley/by-products (WB). Fecal microbiome was characterized by $16 \mathrm{~S}$ ribosomal DNA sequencing, clustered by operational taxonomic unit (OTU), and results were subjected to a discriminant approach combined with principal component analysis to discriminate diets, sexes and FE extreme groups (10 high and 10 low FE pigs for each diet by sex-combination). Pigs on different diets and males vs. females had a very distinct fecal microbiome, needing only 2 OTU for $\operatorname{diet}(P=$ $0.020)$ and 18 OTU for sex $(P=0.040)$ to separate the groups. The 2 most important OTU for diet, and the most important OTU for sex, were taxonomically classified as the same bacterium. In pigs fed the CS diet there was no significant association between FE and fecal microbiota composition based on OTU $(P>0.05)$, but in pigs fed the WB diet differences in FE were associated with 17 OTU in males $(P=0.018)$ and to 7 OTU in females $(P=0.010)$, with 3 OTU in common for both sexes. In conclusion, our results showed a diet and sex dependent relationship between $\mathrm{FE}$ and the fecal microbial composition at slaughter weight in grower-finisher pigs.
\end{abstract}

Keywords: feed efficiency, fecal microbiome, diet, sex, pig 


\subsection{Introduction}

In pork production, feed efficiency (FE) is very important, as feed is the main component of the cost prize. The gut microbiota can play an important role in $\mathrm{FE}$, as pigs do not produce digestive enzymes that allow them to digest the fiber fraction in the diet. Instead, they depend on microbiota residing in the gastrointestinal tract, in particular in the hindgut, to break down the dietary fiber in fermentation processes. VFA are resulting by-products of the fermentation activity of the microbiota and they serve, after absorption from the gut, as energy sources in systemic metabolism (Ingerslev et al., 2014). In pigs, efficiency of energy utilization is lower when energy comes from fiber instead of starch (Noblet and Le Goff, 2001). Thus, for improving FE in pigs, low fiber, high starch diets have been favored (Zijlstra and Beltranena, 2013). However, dietary fiber has shown to reduce stereotypic behavior and aggression (Meunier-Salaün et al., 2001) and improve fecal consistency (Mateos et al., 2006; Wellock et al., 2008). Combined with the increasing competition of feed with human edible products for amongst others arable land (Van Kernebeek et al., 2016), this has caused the agricultural sector to move increasingly towards the formulation of diets with higher fiber contents. Therefore, the importance of intestinal microbiota and their fermentation activity in relation to FE in pigs is likely to increase.

The aim of this study was to investigate the association between FE and fecal microbial composition in commercial grower-finisher pigs. In addition, two factors affecting FE were investigated for their effect on the fecal microbiome: diet composition and sex. 


\subsection{Materials and methods}

This study was carried out in strict accordance with the recommendations in the European Guidelines for accommodation and care of animals. The protocol was approved by the Animal Care and Use Committee of Schothorst Feed Research, The Netherlands (Protocol Number: AVD 246002015120/132). The dataset is available on request from the corresponding author.

\subsubsection{Animals and experimental design}

Pigs used in this study originated from a three-way cross (Synthetic boar $x$ (Large White $x$ Landrace)). Phenotypic data were available for 160 three-breed cross pigs, 81 males and 79 females, coming from 20 litters. All pigs were kept at the experimental facilities of Schothorst Feed Research B.V. (Lelystad, The Netherlands) under commercial conditions. Up until the start of the trial the animals were housed per litter and all animals were fed the same diet. The pigs were put on test at 8 to 9 wk of age (day 0 ), in two groups of 80 , and experimental groups were set 13 wk apart. Distribution was as follows: ten pigs per pen and eight pens per compartment; one compartment was used per entrance date. Littermates were split randomly over the two diets and sexes were housed in separate pens, resulting in two pens per diet per sex per entrance date. All animals were used for the evaluation of the effects of diet composition and sex on fecal microbiota composition, except for six animals of which no fecal sample was obtained. The FE was defined as the ratio of body weight gain to cumulated feed intake from start of the test until the day of slaughter. For evaluation of the effect of fecal microbiota composition on FE the $25 \%$ pigs with the highest and the $25 \%$ with the lowest individual FE per diet per sex (20 animals per combination) were used. Data of one animal were excluded, since it had a very low feed intake and body weight gain during the second half of the test. At the start of the experiment, the pigs had an average BW of $23.0 \mathrm{~kg}$ and were kept in the facilities until they reached a live weight at slaughter of approximately $120 \mathrm{~kg}$. Pigs were allowed a minimal space of $1 \mathrm{~m}^{2}$ per pig, and the pens were equipped with $60 \%$ concrete floor and $40 \%$ slatted floor.

\subsubsection{Feeding Strategy}

Two different diets were studied, a diet based on corn/soybean meal (CS) as typically fed to commercial grower-finisher pigs in The America's and a diet based on wheat/barley/by-products (WB) as typically fed in Europe (Table 3.1). For both diets, the pigs were fed ad libitum according to a three-phase feeding program. The first phase ( $\left.T_{\text {starter }}\right)$ was from days 0 to 25 on test and pigs were fed a starter diet. The 
second phase ( $T_{\text {grower }}$ ) was from days 26 to 67 on test and pigs were fed a grower diet. The third phase $\left(T_{\text {finisher }}\right)$ was from day 68 on test until the pigs reached slaughter weight and they were fed a finisher diet. The diets were custom made diets based on commonly used commercial diets and were formulated on a fixed ratio of net energy to digestible lysine (NE:SID lysine). Each of the three phases had a different $\mathrm{NE}: \mathrm{SID}$ lysine, being $0.89 \mathrm{~J} / \mathrm{g}$ at $\mathrm{T}_{\text {starter, }} 1.06 \mathrm{~J} / \mathrm{g}$ at $\mathrm{T}_{\text {grower }}$ and $1.37 \mathrm{~J} / \mathrm{g}$ at $\mathrm{T}_{\text {finisher. }}$. The increase of NE:SID lysine in grower and finisher diets was mainly achieved by exchanging soybean meal with corn, and peas with wheat for the CS and WB diets respectively. The experimental diets were produced in the feed plant of ABZ Animal Nutrition, Leusden, The Netherlands.

\subsubsection{Measurements and Sampling}

The experimental facilities of Schothorst Feed Research B.V. were equipped with IVOG feeding stations (INSENTEC, Marknesse, The Netherlands) that register individual feed intake of group housed animals. All animals had ear tags with unique incremental numbering, therefore, individual feed intake records were available for all pigs for each day on test. Animals were weighted at days 0,56 and at the end of the test. At the end of the feeding trial ( $1 \mathrm{~d}$ before slaughter), individual fecal samples were collected directly at defecation by hand, with gloves, mixed in the glove and put in small tubes. The samples were immediately frozen in liquid nitrogen and stored at $-80^{\circ} \mathrm{C}$. The ADFI was calculated as the cumulated individual feed intake records throughout the trial divided by the length of the trial. The ADG was calculated as the difference between BW measurements divided by the duration of the trial.

\subsubsection{Fecal Microbiota Analysis}

Fecal samples were used for ribosomal 16S DNA gene sequencing and analysis. Bead beating lyzed the microbial cells and the DNA was purified using the ZR-96 Soil Microbe DNA kit (Zymo Research, Irvine, CA) according to the manufacturer description (Frese et al., 2015). The V3-V4 region was amplified from purified $\begin{array}{lllll}\text { genomic } & \text { wNA th } & \text { F343 }\end{array}$ (CTTTCCCTACACGACGCTCTTCCGATCTTACGGRAGGCAGCAG) and R784 (GGAGTTCAGACGTGTGCTCTTCCGATCTTACCAGGGTATCTAATCCT) using 30 amplification cycles with an annealing temperature of $65^{\circ} \mathrm{C}$ (an amplicon of $510 \mathrm{bp}$, although length varies depending on the organisms). Full length reads of the V3-V4 region were obtained using Illumina Miseq 250-bp paired end reads. Single multiplexing was performed using in house $6 \mathrm{bp}$ index, which were added to R784 during a second PCR with 12 cycles using forward primer 
Table 3.2 Ingredient and calculated nutrient composition of the diets, as-fed basis

\begin{tabular}{|c|c|c|c|c|c|c|}
\hline \multirow[b]{2}{*}{ Item } & \multicolumn{2}{|c|}{$\begin{array}{l}\text { Starter (d } 0 \text { to } \\
25 \text { ) }\end{array}$} & \multicolumn{2}{|c|}{$\begin{array}{c}\text { Grower (d } 26 \text { to } \\
67 \text { ) }\end{array}$} & \multicolumn{2}{|c|}{$\begin{array}{l}\text { Finisher ( } d 68 \text { to } \\
\text { end) }\end{array}$} \\
\hline & CS & WB & CS & WB & CS & WB \\
\hline \multicolumn{7}{|l|}{ Ingredient, $\mathrm{g} / \mathrm{kg}$} \\
\hline Corn & 647.1 & - & 698.4 & - & 755.1 & - \\
\hline Corn gluten & 18.1 & - & 25.0 & 50.0 & 50.0 & 50.0 \\
\hline Soybean meal & 240.5 & 100.0 & 180.5 & 21.5 & 98.3 & - \\
\hline Soybean hull & - & - & - & 14.3 & - & 50.0 \\
\hline Soybean oil & - & 25.0 & - & 0.3 & - & - \\
\hline Barley & - & 200.0 & - & 100.0 & - & 150.0 \\
\hline Wheat & - & 321.9 & - & 400.0 & - & 350.0 \\
\hline Wheat middlings & - & - & - & 50.0 & - & 125.0 \\
\hline Rapeseed meal & - & 63.0 & - & 80.0 & - & 100.0 \\
\hline Sunflower meal & - & 80.0 & - & 80.0 & - & 21.9 \\
\hline Palmkernel meal & - & - & - & 50.0 & - & 50.0 \\
\hline Palm oil & 5.0 & 17.3 & 5.0 & 16.0 & 5.0 & 5.0 \\
\hline Peas & - & 120.0 & - & 29.4 & - & - \\
\hline Sugarcane molasses & 40.0 & 30.0 & 50.0 & 50.0 & 50.0 & 50.0 \\
\hline Animal fat & - & - & - & 27.5 & - & 29.4 \\
\hline Monocalcium phosphate & 6.7 & 5.3 & 2.0 & - & 0.7 & - \\
\hline Salt & 2.7 & 2.1 & 2.4 & 1.8 & 1.8 & 2.1 \\
\hline Calcium carbonate & 11.6 & 10.9 & 9.4 & 8.9 & 9.9 & 4.0 \\
\hline Sodium bicarbonate & - & 1.1 & 1.0 & 1.0 & 3.4 & - \\
\hline Phytase & 5.0 & 5.0 & 5.0 & 5.0 & 5.0 & 1.9 \\
\hline L-Lysine $\mathrm{HCl}$ & - & 3.8 & - & 4.3 & - & - \\
\hline DL-Methionine & - & 1.3 & - & 0.7 & - & - \\
\hline L-Threonine & - & 1.7 & - & 1.6 & - & - \\
\hline Lysine + Thrypophan & 7.7 & 4.3 & 8.2 & 3.6 & 9.1 & - \\
\hline Lysine $\mathrm{HC}$ & 3.0 & - & 2.7 & - & 2.3 & 4.0 \\
\hline Methionine $\mathrm{HC}$ & 2.8 & - & 2.5 & - & 1.5 & 0.3 \\
\hline Threonine $\mathrm{HC}$ & 3.8 & - & 3.9 & - & 3.8 & 2.4 \\
\hline Valine & - & 1.4 & - & - & - & - \\
\hline Vitamin premix ${ }^{1}$ & 0.1 & 0.1 & & & & \\
\hline $\begin{array}{l}\text { Vitamin-trace mineral } \\
\text { premix } 1^{2}\end{array}$ & 0.1 & 0.1 & & & & \\
\hline $\begin{array}{l}\text { Vitamin-trace mineral } \\
\text { premix } 2^{3}\end{array}$ & 0.4 & 0.4 & 0.4 & 0.4 & 0.4 & 0.4 \\
\hline
\end{tabular}




\section{Fecal microbiome and feed efficiency in pigs}

$\begin{array}{lcccccc}\text { Nutrient composition, } \mathrm{g} / \mathrm{kg}^{4} & & & & & & \\ \text { NE, MJ/kg } & 9.9 & 9.9 & 10.1 & 9.7 & 10.3 & 9.3 \\ \text { Moisture } & 127 & 126 & 130 & 126 & 130 & 129 \\ \text { Ash } & 51 & 52 & 42 & 47 & 38 & 42 \\ \text { Crude protein } & 182 & 190 & 159 & 166 & 128 & 147 \\ \text { Crude fat } & 34 & 58 & 35 & 64 & 36 & 57 \\ \text { Crude fibre } & 24 & 45 & 24 & 60 & 25 & 71 \\ \text { Starch } & 437 & 360 & 471 & 335 & 512 & 334 \\ \text { Sugar } & 44 & 50 & 46 & 58 & 42 & 59 \\ \text { NSP } & 135 & 170 & 130 & 216 & 126 & 246 \\ \text { Ca } & 6.9 & 6.9 & 5.2 & 5.5 & 5.0 & 3.8 \\ \text { P } & 4.8 & 5.5 & 3.6 & 4.7 & 3.2 & 4.7 \\ \text { SID Lys } & 11.1 & 11.1 & 9.5 & 9.1 & 7.5 & 6.8 \\ \text { SID Met + Cys } & 6.6 & 6.6 & 5.9 & 5.6 & 4.6 & 4.6 \\ \text { SID Thr } & 7.1 & 7.1 & 6.3 & 6.0 & 5.2 & 4.7 \\ \text { SID Trp } & 2.1 & 2.1 & 1.8 & 1.7 & 1.4 & 1.3\end{array}$

${ }^{1}$ Supplied per kilogram of feed: $2500 \mathrm{IU}$ of vitamin A, $500 \mathrm{IU}$ of vitamin D3, and 5 IU of vitamin E (Mervit AD3E; PreMervo, Utrecht, the Netherlands).

2Supplied per kilogram of feed: $12 \mathrm{mg}$ of Fe (ferrous sulfate), $10 \mathrm{mg}$ of $\mathrm{Mn}$ (manganous oxide), $0.04 \mathrm{mg}$ of Co cobalt oxide), $0.12 \mathrm{~g}$ of $\mathrm{Ca}, 0.0501 \mathrm{~g}$ of $\mathrm{P}, 0.04 \mathrm{mg}$ of I (potassium iodide), 1000 IU of vitamin A, $100 \mathrm{IU}$ of vitamin D3, $5 \mathrm{IU}$ of vitamin $\mathrm{E}, 0.4 \mathrm{mg}$ of vitamin B1, $0.8 \mathrm{mg}$ of vitamin B2, $2 \mathrm{mg}$ of pantothenic acid, $4 \mathrm{mg}$ of niacine, $0.4 \mathrm{mg}$ of vitamin B6, $0.2 \mathrm{mg}$ of folate, $0.003 \mathrm{mg}$ of vitamin B12, $10 \mathrm{mg}$ of vitamin C, $0.01 \mathrm{mg}$ of biotine, $0.2 \mathrm{mg}$ of vitamin $\mathrm{K} 3$, and $40 \mathrm{mg}$ of choline (Mervit Sporavit; PreMervo).

${ }^{3}$ Supplied per kilogram of premix: $0.4 \mathrm{~g}$ of $\mathrm{Ca}, 15 \mathrm{mg}$ of $\mathrm{Cu}$ (copper sulfate) $0,80 \mathrm{mg}$ of $\mathrm{Fe}$ (ferrous sulfate), $24 \mathrm{mg}$ of $\mathrm{Mn}$ (manganous oxide), $62 \mathrm{mg}$ of $\mathrm{Zn}$ (zinc oxide), $0.04 \mathrm{mg}$ of Co (cobalt oxide), $0.4 \mathrm{mg}$ of I (potassium iodide), $0.2 \mathrm{mg}$ of Se (sodium selenite), $7500 \mathrm{IU}$ of vitamin A, 1500 IU of vitamin D3, 25 IU of vitamin E, $4 \mathrm{mg}$ of vitamin B2, $6 \mathrm{mg}$ of pantothenate, $30 \mathrm{mg}$ of niacin, $0.02 \mathrm{mg}$ of vitamin B12, and $0.752 \mathrm{mg}$ of vitamin K3 (Mervit START M220; PreMervo, Utrecht, the Netherlands).

${ }^{4}$ Based on chemical composition, digestibility, and energy values for pigs from the Centraal Veevoeder Bureau livestock feed table (Centraal Veevoeder Bureau, 2019). 


\section{Fecal microbiome and feed efficiency in pigs}

(AATGATACGGCGACCACCGAGATCTACACTCTTTCCCTACACGAC) and reverse primer (CAAGCAGAAGACGGCATACGAGAT-index-GTGACTGGAGTTCAGACGTGT). The resulting $P C R$ products were purified and loaded onto the Illumina MiSeq cartridge according to the manufacturer instructions. The quality of the run was checked internally using PhiX control as recommended by manufacturer, and then each pairend sequence was assigned to its sample with the help of the previously integrated index. Each pair-end sequence was assembled using Flash software (Magoč and Salzberg, 2011) using at least a 10bp-overlap between the forward and reverse sequences, allowing $10 \%$ of mismatch (Lluch et al., 2015). The absence of contamination was checked with a negative control during the PCR (water as template). The quality of the stitching procedure was controlled using four bacterial samples that are run routinely in the sequencing facility in parallel to the current samples.

\subsubsection{Statistical analysis}

The resulting sequences of the 154 samples were clustered with Usearch (Edgar, 2010) using the Uparse pipeline (Edgar, 2013) to create operational taxonomic units (OTU). The OTU table of abundance was analyzed by discriminant analysis using principal components (DAPC) (Jombart et al., 2010), to test the association of OTU abundance with a number of factors. Number of dimensions to be included in further analyses was chosen based on stability of the results, determined by adding increasingly more dimensions. In case the stability test gave a range of dimensions, a threshold value of $99 \%$ of the original variance was used to decide the number of dimensions. The OTU were sorted based on their contribution to the separation of tested factors in the discriminant analysis, which echoes the weight of each OTU in separating the groups. Using this order, increasingly more OTU were added to separate the groups, until the separation reached significance at $P<0.05$. The builtin a-score method of the DAPC was used to determine the statistical significance of the separation based on a permutation test. Briefly, 1,000 simulations with randomized group labels were performed to evaluate if the discriminant analysis could separate the samples in any of those random configurations. The a-score obtained with the true groups was compared to the distribution of the a-scores obtained with the 1000 simulations to determine if the separation was due to chance (Jombart et al., 2010). The method was repeated to test separation for diet, sex, and FE groups, and to test association of phylum, class and genera abundance (based on OTU taxonomy) with these factors. To test whether the results extrapolated to the whole dataset, the OTU relevant for separating the FE groups were used for partial least squares regression (PLSr)(Mevik and Wehrens, 2007) on all animals within the 
groups and not only the FE extreme pigs. The number of components kept was based on the lowest root-mean-squares error of prediction after leave-one-out cross validation.

After rarefying the data (McMurdie and Holmes, 2013), Bray-Curtis distances between diet, sex and FE extreme groups were calculated using a maximum of 200 iterations for diet and sex and 100 iterations for FE groups and tested with ADONIS for significance (Oksanen et al., 2017). Shannon Index, Simpson diversity index and chao1 richness estimator were calculated using the vegan package (Oksanen et al., 2017). Significance of difference in the diversity estimates between the diets, sexes and FE groups was determined using a generalized linear model (SAS 9.3; SAS Inst. Inc., Cary, NC) with diet, sex, and FE groups as fixed effect. Significance of difference in $A D G, A D F I$, and FE between the high and low FE groups was determined using a mixed model (SAS 9.3; SAS Inst. Inc., Cary, NC) with animal as experimental unit, FE groups and pen as fixed effect, and BW at start of the test as covariable. For the least squares means calculations BW at start of the test was fixed at $22 \mathrm{~kg}$.

\subsubsection{Taxonomy}

To investigate biological functionality of differences between groups, the taxonomy was determined for each OTU by the SILVA Incremental Aligner (SINA) software (Pruesse et al., 2012), which aligns the OTU with the rRNA gene databases provided by the SILVA ribosomal RNA project (Quast et al., 2013). Default SINA settings were used to assign the taxonomy of each OTU, with the minimum identity with query sequence set at 0.97 and number of neighbors per query sequence set at 10 . Group level information within genera classification was deleted. In addition, OTU found by DAPC analysis were blasted against the NCBI 16S ribosomal RNA sequences (Bacteria and Archeae) database using BLASTn (McGinnis and Madden, 2004) to determine the bacteria with closest sequence similarity. Default megaBLAST settings were used.

\subsection{Results}

Within the DAPC analysis, it is not possible to account for the pen effect directly. However, when doing a DAPC analysis for all the piglets across all the pens, the cohoused piglets did not group together (results not shown).

\subsubsection{Differences Between Diets}

Between the two diets, differences in the relative abundance of the nine major phyla, classes, and genera for both diets were observed (Fig. 3.1). This was reflected in the Bray-Curtis distances at phylum and OTU level (Fig. 3.2), which were 
significantly different $(P<0.001)$, but not on class and genera level. The DAPC analysis gave a clear separation in fecal microbiota composition between the two diets based on phyla, classes, genera and OTU (Fig. 3.3) $(P<0.001)$. The separation was based on 3, 4, 10 and 55 dimensions for phyla, classes, genera and OTU respectively, which represented at least $99 \%$ of the original variance in microbiota composition. Keeping the two phyla (Bacteroidetes and Proteobacteria), three classes (Gammaproteobacteria, Spirochaetes, and Bacteroidia), two genera (Ruminococcus and Blautia), and two OTU (OTU 33 and OTU 16) with the highest contribution to the separation was sufficient to discriminate pigs on different diets. Blasting the sequence of the two most contributing OTU to NCBI gave a $95 \%$ identity with $99 \%$ query coverage with the bacterium Butyricicoccus pullicaecorum. The second most important OTU resulted in the same bacterium, with $96 \%$ identity and 99\% query coverage. This difference between the diets, however, was not depicted in the measures for diversity. The CS diet had a higher Shannon index than the WB diet $(P=0.021)$, but the Simpson index and the chao1 index were similar for both diets.
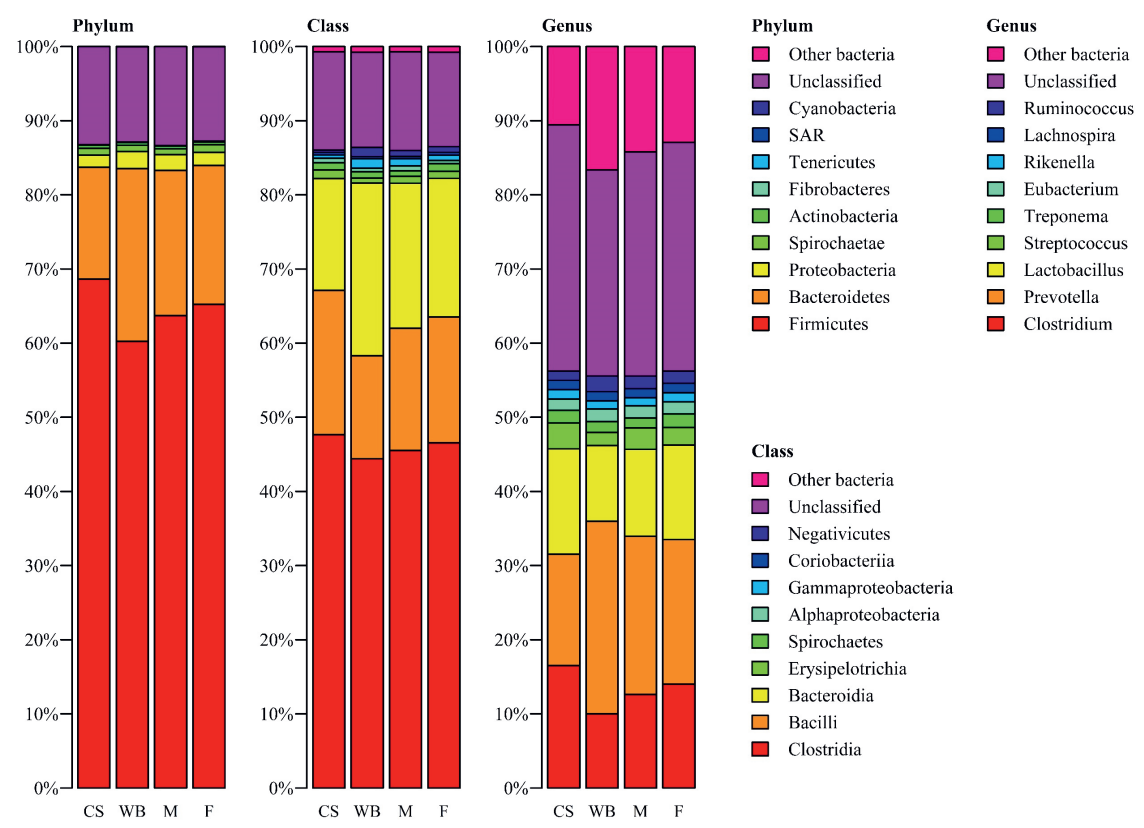

Figure 3.1 Relative abundance of 9 major bacterial phyla, classes, and genera in the feces male (M) and female (F) of pigs fed a corn/soybean meal diet (CS) or a wheat/barley/by-products $\operatorname{diet}(\mathrm{WB})$. Data are mean percentage of total identified sequences. 

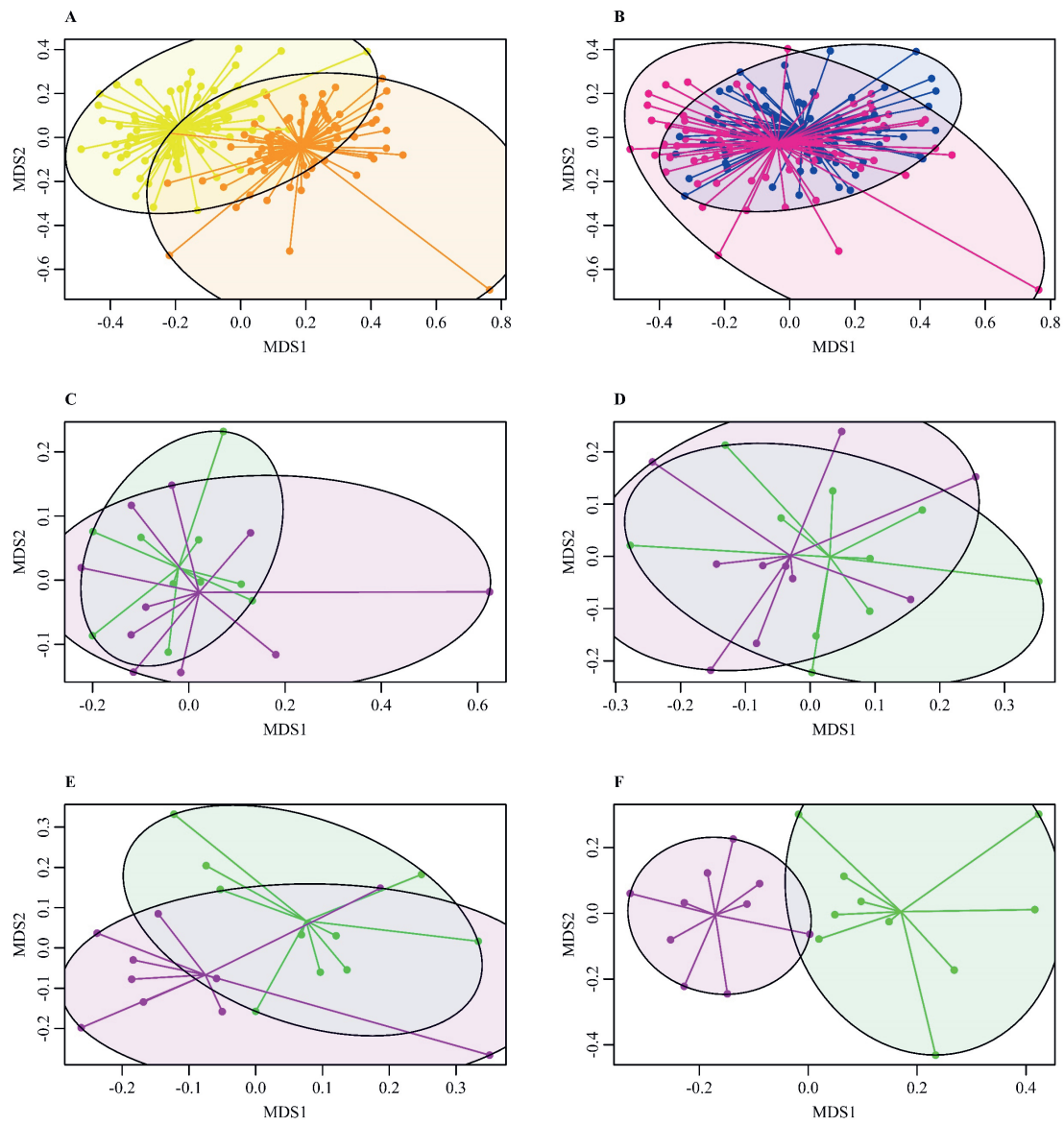

Figure 3.2 Bray-Curtis distances for tested groups based on operational taxonomic units. (A) Diet. Yellow $=$ corn $/$ soybean meal diet, orange $=$ wheat $/$ barley $/$ by-products diet. $(B)$ Sex. Blue $=$ male pigs, pink $=$ female pigs. $(C-F)$ Feed efficiency. Green $=$ high feed efficiency, purple $=$ low feed efficiency. (C) Male pigs fed a corn/soybean meal diet, (D) female pigs fed a corn/soybean meal diet, (E) male pigs fed a wheat/barley/by-products diet, and (F) female pigs fed a wheat/barley/by-products diet. 

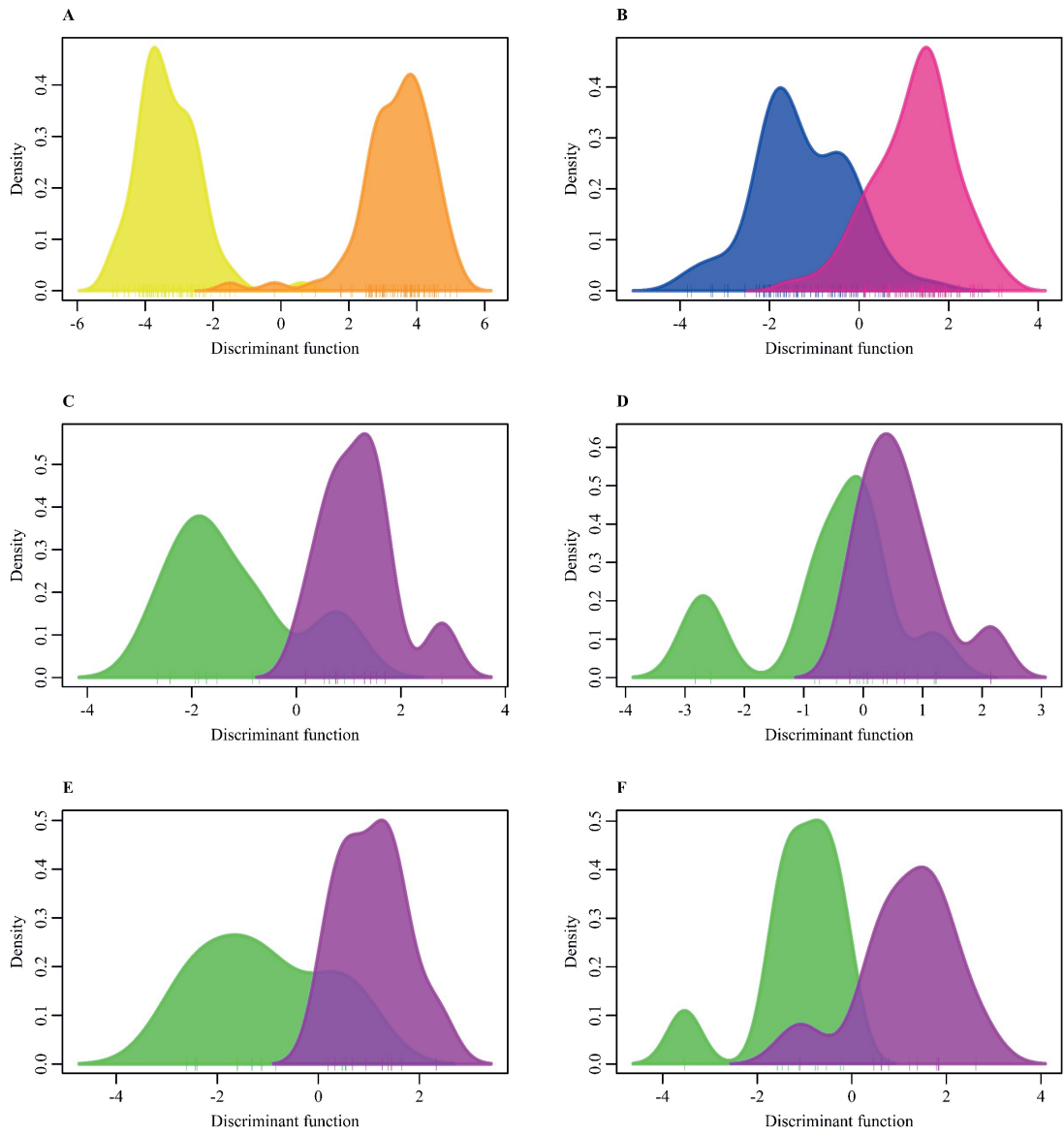

Figure 3.3 Gaussian kernel density estimation of the discriminant function as result of the discriminant analysis of principle components for tested groups based on operational taxonomic units. (A) Diet. Yellow $=$ corn $/$ soybean meal diet, orange $=$ wheat $/$ barley $/$ by products diet. $(B)$ Sex. Blue $=$ male pigs, pink $=$ female pigs. $(C-F)$ Feed efficiency. Green $=$ high feed efficiency, purple $=$ low feed efficiency. (C) Male pigs fed a corn/soybean meal diet, (D) female pigs fed a corn/soybean meal diet, (E) male pigs fed a wheat/barley/by-products diet, (F) female pigs fed a wheat/barley/by-products diet. 


\subsubsection{Differences Between Sexes}

In contrast to the diets, the overview of the relative abundance of the nine major phyla, classes, and genera (Fig. 3.1) does not indicate obvious differences between the sexes. This is reflected by the results of the Bray-Curtis distances, which were only significant at OTU level ( $P=0.037$ ) (Fig. 3.2). The DAPC analysis gave somewhat similar results, as it indicated no separation between the two sexes based on phyla (7 dimensions) and needed 22 out of 45 classes to reach a significant difference between the male and female pigs using 16 dimensions and $100.0 \%$ of the original variance. However, there was a highly significant distinction for sex based on genera $(P=0.003)$ and OTU ( $P=0.001)$ (Fig. 3.3), based on 38 and 60 dimensions $(100.0 \%$ and $99.2 \%$ of the original variance), respectively. There were six genera and 18 OTU required to reach a significant separation between sexes. For nine out of those 18 OUT, it was possible to reliably assign the genus, for 8 it was possible to reliably assign the family, and for one OTU it was not possible to assign any taxonomy (Table

Table 3.2 Abundancy and taxonomy (genus level) of the OTU in order of statistical contribution to the separation between sexes

\begin{tabular}{|c|c|c|c|}
\hline \multirow[b]{2}{*}{ OTU ID } & \multirow[b]{2}{*}{ Classification } & \multicolumn{2}{|c|}{ Percentage of total sequences } \\
\hline & & Boar & Gilt \\
\hline OTU16 & Unclassified Ruminococcaceae $^{1}$ & 0.57 & 0.38 \\
\hline OTU35 & Unclassified Ruminococcaceae ${ }^{1}$ & 0.84 & 0.76 \\
\hline OTU12472 & Clostridium & 0.77 & 0.77 \\
\hline OTU373 & Subdoligranulum & 0.16 & 0.23 \\
\hline OTU191 & Unclassified $^{2}$ & 0.05 & 0.14 \\
\hline OTU174 & Unclassified Bacteroidales ${ }^{1}$ & 0.05 & 0.15 \\
\hline OTU22 & Roseburia & 0.26 & 0.33 \\
\hline OTU71 & Ruminococcus & 0.15 & 0.21 \\
\hline OTU33 & Unclassified Ruminococcaceae ${ }^{1}$ & 0.28 & 0.27 \\
\hline OTU19 & Coprococcus & 0.34 & 0.35 \\
\hline OTU136 & Prevotella & 0.06 & 0.13 \\
\hline OTU29 & Unclassified Succinivibrionaceae $^{1}$ & 0.28 & 0.32 \\
\hline OTU20 & Ruminococcus & 0.39 & 0.42 \\
\hline OTU8 & Turicibacter & 0.88 & 0.91 \\
\hline OTU38 & Unclassified Prevotellaceae ${ }^{1}$ & 0.44 & 0.42 \\
\hline OTU127 & Unclassified Prevotellaceae ${ }^{1}$ & 0.20 & 0.13 \\
\hline OTU1050 & Unclassified Prevotellaceae ${ }^{1}$ & 0.42 & 0.32 \\
\hline OTU44 & Ruminococcus & 0.32 & 0.19 \\
\hline
\end{tabular}

${ }^{1}$ Reliable depth of taxonomy is limited to family level (query sequence identical for at least 95\%)

${ }^{2}$ No taxonomic classification available (query sequence identical for at least $95 \%$ ) 


\section{Fecal microbiome and feed efficiency in pigs}

3.2). The main class differing between the sexes was Methanobacteria and the main genera differing was Bifidobacterium. The most important OTU for sex separation was the same as for diet, which was associated with B. pullicaecorum. There was no difference in any of the diversity indexes between the sexes.

\subsubsection{Differences Between FE Extremes}

As there was a strong effect of diet and sex on the fecal microbial composition, the dataset was split into 4 groups to estimate the association between FE and microbiome within diet by sex combination. There was a 0.062 to $0.078 \mathrm{~g} / \mathrm{g}$ difference between the FE groups in FE (Table 3.3) and there was no pen effect in any of the groups.

Table 3.3 Least squares means of the high and low FE groups during the experimental period (overall mean $\mathrm{BW}$ at start $=22 \mathrm{~kg}$, overall mean $\mathrm{BW}$ at end $=121 \mathrm{~kg}$ ) per diet by sex combination

\begin{tabular}{|c|c|c|c|c|c|}
\hline \multirow[b]{2}{*}{ Item } & \multicolumn{2}{|c|}{ FE groups } & \multirow[b]{2}{*}{ SEM } & \multicolumn{2}{|l|}{$P$-value } \\
\hline & Low & High & & BW start & FE group \\
\hline \multicolumn{6}{|l|}{$\operatorname{CSM}^{1}$} \\
\hline$A D G, g / d$ & 894 & 1028 & 24 & 0.255 & 0.001 \\
\hline $\mathrm{ADFI}, \mathrm{kg} / \mathrm{d}$ & 2.28 & 2.19 & 0.07 & 0.123 & 0.357 \\
\hline $\mathrm{FE}, \mathrm{g} / \mathrm{g}$ & 0.39 & 0.47 & 0.01 & 0.057 & $<0.001$ \\
\hline \multicolumn{6}{|l|}{$\operatorname{CSF}^{1}$} \\
\hline$A D G, g / d$ & 909 & 1045 & 25 & 0.004 & 0.001 \\
\hline ADFI, kg/d & 2.41 & 2.38 & 0.06 & 0.001 & 0.724 \\
\hline $\mathrm{FE}, \mathrm{g} / \mathrm{g}$ & 0.38 & 0.44 & 0.00 & 0.243 & $<0.001$ \\
\hline \multicolumn{6}{|l|}{$\mathrm{WBM}^{1}$} \\
\hline$A D G, g / d$ & 899 & 1016 & 23 & 0.008 & 0.001 \\
\hline ADFI, kg/d & 2.27 & 2.18 & 0.06 & 0.003 & 0.274 \\
\hline $\mathrm{FE}, \mathrm{g} / \mathrm{g}$ & 0.40 & 0.47 & 0.00 & 0.051 & $<0.001$ \\
\hline \multicolumn{6}{|l|}{$\mathrm{WBF}^{1}$} \\
\hline$A D G, g / d$ & 931 & 992 & 27 & 0.499 & 0.120 \\
\hline $\mathrm{ADFI}, \mathrm{kg} / \mathrm{d}$ & 2.60 & 2.27 & 0.06 & 0.305 & 0.002 \\
\hline $\mathrm{FE}, \mathrm{g} / \mathrm{g}$ & 0.36 & 0.44 & 0.00 & 0.471 & $<0.001$ \\
\hline
\end{tabular}

${ }^{1} \mathrm{CSM}=$ male pigs fed a corn/soybean meal diet

${ }^{2} \mathrm{CSF}=$ female pigs fed a corn/soybean meal diet

${ }^{3} \mathrm{WBM}=$ male pigs fed $\mathrm{a}$ wheat $/$ barley $/$ by -products diet

${ }^{4} \mathrm{WBF}=$ female pigs fed a wheat/barley/by-products diet 
There was no difference in diversity index between the high and low FE animals in any of the diet by sex combinations. In addition, there were only significant BrayCurtis distances at OTU level for the pigs fed the WB diet (Fig. 3.2). Compared with the diet and sex analyses, the separation between the FE groups using the DAPC analysis was not as clear (Fig. 3.3). At phylum level, only the male pigs fed a WB diet could be separated using five dimensions (100.0\% of the original variance). Two phyla were necessary for significant separation, Actinobacteria and Proteobacteria, which were both highest in the high FE pigs. Also at class level, the male pigs fed a WB diet could be significantly separated, based on five dimensions explaining $99.7 \%$ of the original variance. Gammaproteobacteria was the first out of the nine contributing classes used for the separation. In addition, the male pigs fed a CS diet could be significantly separated $(P=0.008)$ and there were16 classes used for the separation. At genera level, the analysis only showed significant separation between high and low female pigs fed the CS diet $(P=0.009)$ and male pigs fed the WB diet $(P$ $=0.038$ ). Four dimensions were used, explaining $98.7 \%$ and $98.3 \%$ of the original variance, respectively, and keeping only two genera was sufficient for the separation in the female pigs. These genera were Prevotella and Streptococcus. There were 11 genera needed for the separation in the male pigs with the main genera being Roseburia.

In the pigs fed the CS diet, there was no significant separation for either of the sexes when using OTU, based on eight dimensions for male animals and three for females, explaining $96.7 \%$ and $83.2 \%$ of the original variance, respectively. In the pigs fed the WB diet, when five dimensions were used ( $82.7 \%$ of the original variance), the low FE $(P=0.016)$, but not the high FE $(P=0.690)$, could be identified in the pool of males. In the females fed the WB diet, the high FE pigs were identified $(P=0.016)$, but not the low FE animals $(P=0.094)$, based on five dimensions $(87.8 \%$ of the original variance). In total, 17 OTU were necessary to discriminate the low FE male pigs ( $P=$ 0.018) (Table 3.4) and seven OTU to distinguish the high FE female pigs ( $P=0.010)$ fed a WB diet (Table 3.5). Putting these OTU in PLSr resulted in an $R^{2}$ of 0.14 (2 components) and 0.11 ( 3 components) for male and female pigs fed the WB diet, respectively (Fig. 3.4). Three of the OTU significant for discriminating high and low FE pigs were common for the male and female pigs. Strikingly, the effects of OTU 4 and 2 had different directions in male and female pigs, as higher abundance was associated with high FE in males and low FE in females. 


\section{Fecal microbiome and feed efficiency in pigs}

Table 3.4 Abundancy and taxonomy (genus level) of the OTU in order of statistical contribution to the separation between high and low FE boars fed a wheat/barley/by-product diet

\begin{tabular}{lccc}
\hline \multirow{2}{*}{ OTU ID } & & \multicolumn{2}{c}{ Percentage of total sequences } \\
\cline { 2 - 4 } & Classification & Low FE & High FE \\
\hline OTU4 & Lactobacillus & 1.75 & 4.36 \\
OTU24 & Roseburia & 0.23 & 1.36 \\
OTU2 & Unclassified Peptostreptococcaceae $^{1}$ & 4.59 & 5.10 \\
OTU12 & Unclassified Prevotellaceae $^{1}$ & 0.89 & 1.34 \\
OTU3 & Lactobacillus & 1.71 & 1.60 \\
OTU244 & Prevotella & 3.96 & 2.60 \\
OTU5 & Streptococcus & 1.80 & 2.41 \\
OTU8955 & Roseburia & 0.01 & 0.45 \\
OTU1050 & Prevotella & 0.95 & 0.16 \\
OTU9 & Roseburia & 6.43 & 4.73 \\
OTU3132 & Clostridium & 0.03 & 0.43 \\
OTU1 & Roseburia & 8.96 & 7.28 \\
OTU22 & Clostridium & 0.29 & 0.67 \\
OTU12472 & Ruminococcus & 0.47 & 0.95 \\
OTU41 & Roseburia & 1.82 & 1.34 \\
OTU180 & Unclassified Prevotellaceae & 0.29 \\
OTU13 & Rrevotellaceae & 2.50 \\
\hline
\end{tabular}

${ }^{1}$ Reliable depth of taxonomy is limited to family level (query sequence identical for at least 95\%)

Table 3.5 Abundancy and taxonomy (genus level) of the OTU in order of statistical contribution to the separation between high and low FE gilts fed a wheat/barley/by-product diet

\begin{tabular}{lccc}
\hline \multirow{2}{*}{ OTU ID } & & \multicolumn{2}{c}{ Percentage of total sequences } \\
\cline { 3 - 4 } & Classification & Low FE & High FE \\
\hline OTU2 & Unclassified Peptostreptococcaceae $^{1}$ & 5.35 & 5.27 \\
OTU10 & Prevotella & 0.65 & 2.00 \\
OTU55 & Ruminococcus & 0.24 & 0.82 \\
OTU13 & Roseburia & 2.80 & 1.76 \\
OTU4 & Lactobacillus & 4.25 & 3.14 \\
OTU49 & Prevotella & 0.97 & 0.32 \\
OTU6 & Lactobacillus & 4.21 & 2.45
\end{tabular}

${ }^{1}$ Reliable depth of taxonomy is limited to family level (query sequence identical for at least 95\%) 

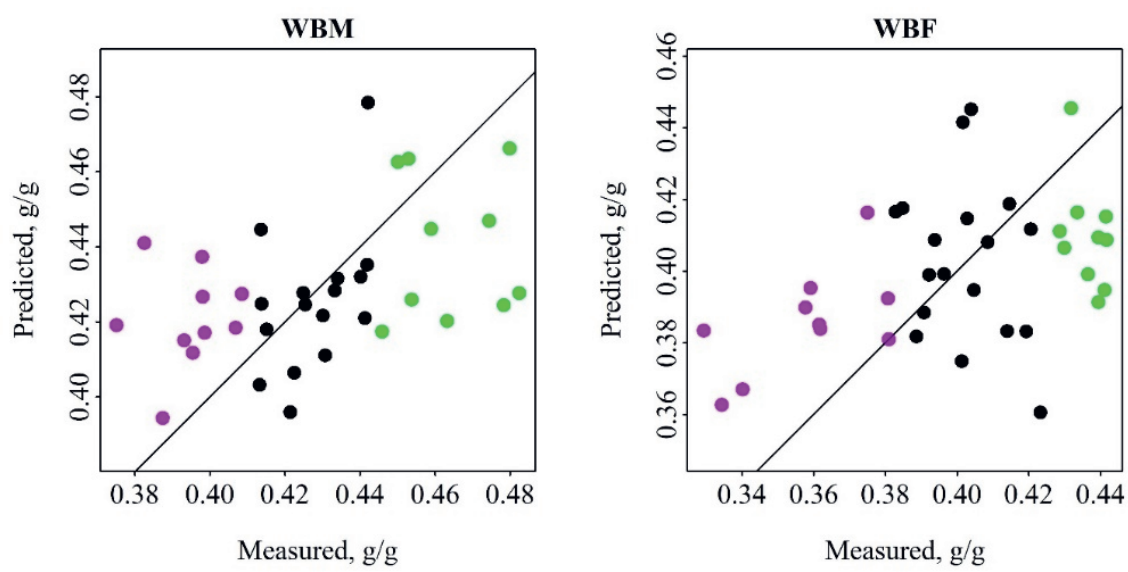

Figure 3.4 Measured vs. predicted feed efficiency by partial least squares regression based on significant operational taxonomic units found by discriminant analysis of principal components. (A) Male pigs fed a wheat/barley/by-products diet. $R^{2}=0.14$. (B) Female pigs fed a wheat/barley/by-products diet. $R^{2}=0.11$. Green $=$ pigs in high feed efficiency group, purple $=$ pigs in low feed efficiency group.

\subsection{Discussion}

The aim of this study was to investigate the association between FE and the composition of the fecal microbiome in commercial grower-finisher pigs. In the present experiment, the fecal microbiome was used as an indicator for the microbiome in the gastro-intestinal tract during the whole grower-finisher period. However, extrapolation of results of the microbial composition in the feces to other compartments of the gastro-intestinal tract might not be valid. Microbial composition in the ileum, cecum, and colon differs, with the ileal intestinal microbiome being most different from that in other compartments (Looft et al., 2014). Moreover, microbial composition in digesta in the lumen of the gut is different from the mucosa associated microbiota (Looft et al., 2014). It is also questionable whether the fecal samples, taken at the end of the grower-finisher period, are representative for the whole grower finisher period, as the microbial composition in the feces might change with age (Kim et al., 2011). As the microbial composition at the start and at other time points of the experiment was not measured, the age at which differences in the microbiome for the tested effects appear is unknown. Nevertheless, the fecal microbiome seems most similar to both luminal and mucosal microbiome in the mid-colon (Looft et al., 2014) and is most similar for pigs aged 10 and 13 wk, and for pigs aged 16, 19, and 22 wk (Kim et al., 2011). Therefore, when interpreting our results in terms of relationships between microbial composition and performance of the pig, it should be considered that the fecal microbiome measured 


\section{Fecal microbiome and feed efficiency in pigs}

in the present study is likely most representative for the microbial composition in the colon, in particular during the last part of the growth trajectory considered.

\subsubsection{Diets}

From the literature, it is well known that diet composition affects the microbial composition in the gastrointestinal tract (Bauer et al., 2006) and the current study confirms these observations. Worldwide there are two mainstream diets fed to grower-finishers based on the availability of main ingredients: a diet based on corn and soybean meal as is common in North and South America, and a diet based on wheat, barley, and by-products from the agro-food sector as is common in Europe and parts of China. Both diets are used to grow pigs as fast and cost-efficient as possible, even though the ingredient composition is rather different. The diets studied differed mostly in dietary fiber content and composition. The main fiber components in wheat, barley, and corn are arabinoxylans, $\beta$-glucans, and cellulose, whereas in soybean meal, the fiber mainly contains pectic substances in the form of rhamnogalacturonan (Choct, 1997). This is reflected in the observed differences in microbiome between the two diets in the current study, as B. pullicaecorum, comprising the two OTU with highest abundance in the CS diet, is highly efficient in fermenting starch (Eeckhaut et al., 2008). This most likely relates to the high starch content in the CS diet. Additionally, the third most important OTU was found to be Blautia wexlera and had the highest abundancy in the WB diet. This bacterium mainly ferments arabinose, glucose, mannose and xylose (Liu et al., 2008), which relates to the high arabinoxylans content of the WB diet. So the most contributing OTU to discriminate pigs on the different diets resemble the source of dietary fiber.

\subsubsection{Sexes}

Our results are in accordance with a recent study of Xiao et al. (2016), which also showed a difference between male and female finisher pigs in fecal microbial composition. Both studies found differences in bacteria belonging to the Prevotella and Ruminococcus genus. Previously, most of the research in pigs investigated changes in intestinal microbiota related to digestive problems and diarrhea postweaning in weaners (Konstantinov et al., 2006; Pajarillo et al., 2014). These studies in weaners did not find a sex effect on the microbiome (Mach et al., 2015). Sex steroids hormones might partially explain this, as levels of some sex steroids hormones rapidly increase at onset of puberty (Camous et al., 1985; Zamaratskaia et al., 2004). In mice, gonadectomy of males and females resulted in a change in microbial composition of the feces, but testosterone treatment of the castrated males resulted in a microbiome similar to that of intact males (Org et al., 2016). 
Metabolism residues of sex steroids hormones are excreted through bile into the lumen of the small intestine (Goymann, 2012), resulting in a different bile composition between sexes (Org et al., 2016). Mainly the Firmicutes, Proteobacteria, and Actinobacteria can metabolize and degrade steroid hormones (García-Gómez et al., 2012), which is reflected in the difference in OTU between the sexes in our study, where 11 out of the 18 OTU belonged to the Firmicutes phyla. Other pathways through which sex steroid hormones might influence microbiota are the mucosal immune activation (Sankaran-Walters et al., 2013) and expression of steroid receptors (Menon et al., 2013). The observed limited effect of sex on microbial composition in the feces of weaners and the substantial effect at slaughter age is likely because sex steroid hormones only start to play a large role in finisher pigs.

\subsubsection{Feed Efficiencies}

There are several ways via which the intestinal microbiota could influence FE of pigs, including competition between the host and the microbiota for nutrients in the small intestine and activation of the immune system through stimulation of the development of the mucus layer, epithelial cells, and lamina propria (Dibner and Richards, 2005). The latter could possibly induce changes in nutrient partitioning between utilization for immune system functioning and for deposition e.g. in muscle protein, but this is likely to be primarily a juvenile phenomenon (Dibner and Richards, 2005). In addition, quantitative production of VFA by intestinal microbiota can relate to FE. Approximately $68 \%$ of the gross energy in fermentable carbohydrates can be transformed into VFA (Williams et al., 2001). The VFA composition depends amongst others on the composition of the substrates, microbial composition and activity, and absorption of the VFA across the large intestinal wall (Williams et al., 2001). Butyrate is the preferred energy source for colonocytes, $76 \%$ of the mucosal absorbed butyrate is metabolized in these cells (Herrmann et al., 2011). Once absorbed across the intestinal wall, the VFA are available as precursor and energy substrate in organs and tissues in the body. Propionate is a precursor for glucose and is almost fully extracted by the liver (Ingerslev et al., 2014), whereas acetate and butyrate are used for Acetyl-CoA production. Next to being direct energy substrates, VFA are also involved as regulators in fatty acid, glucose, and cholesterol metabolism (den Besten et al., 2013). Therefore, the microbiota might influence FE by the amount and composition of VFA produced.

There was a significant relationship between microbiome and FE in pigs fed the WB diet, but there was no significant relationship in pigs fed the CS diet on OTU level. The fiber level in the diets might explain this difference. When assuming the VFA 


\section{Fecal microbiome and feed efficiency in pigs}

production to contribute to the $\mathrm{FE}$ of the pigs, the difference in performance between the high and low FE pigs due to microbial composition differences is expected to be more pronounced at a higher content of fermentation substrate in the diet. As the finisher WB diet contained 2.8 times more crude fiber than the CS diet, there was more substrate available for fermentation in the WB diet. Consequently, in our study, the amount of substrate available might not have been sufficient to detect a relationship between microbiome and FE in the pigs fed the CS diet, whereas it was sufficient in the pigs fed the WB diet.

In male pigs fed the WB diet, the most contributing OTU to separate the FE groups was taxonomically classified as Lactobacillus, the high FE group having a higher abundance of this OTU. In contradiction to our results, Vigors et al. (2016b) only showed a difference in Lactobacilli spp. in the cecum, and not in the colon, between divergent groups in residual feed intake in pigs. Nevertheless, the direction of the effect was similar in both studies, with an increase in Lactobacillus having a positive effect on FE. The species related to this OTU only produce D- and L-lactate (Roos et al., 2005; Slavica et al., 2015). In contrast, in the female pigs fed the WB diet, the same OTU was higher in the low FE group, but the difference was smaller between the FE groups in the female pigs. In accordance with the results of McCormack et al. (2017), the Clostridium abundancy in feces was important to distinguish between the high and low FE pigs. However, this was only the case in the male pigs fed the WB diet, and the two OTU classified as Clostridium had opposite effects. In addition, the other five genera important for distinguishing pigs divergent in residual feed intake discovered by McCormack et al. (2017) were not found in our study. An explanation may lie in the difference between the diets of the studies. Everything considered, the microbiota associated with FE in grower-finisher pigs might consist of several crucial species and other species only relevant in certain situations, e.g., when certain diets are fed.

\subsubsection{Implications}

Results of the present study suggest possibilities to improve FE of grower-finisher pigs by altering microbial composition in the distal part of the intestinal tract. Modification of diet composition might be an option to change microbiota composition, e.g., by changing fiber source or inclusion level, or by including specific additives such as probiotics, prebiotics, organic and inorganic acids, and essential oils (De Lange et al., 2010). In summary, FE might be improved by changing the nutrition of pigs partly through resulting changes in microbiota composition. 


\subsection{Conclusion}

There is a sex dependent relationship between the fecal microbial composition and FE in grower-finisher pigs fed a WB diet, having a higher concentration of dietary fiber than a CS diet. The exact interplay between the fecal microbial composition, composition and concentration of fiber, and production of VFA by intestinal microbiota remains to be determined. Furthermore, results on the relationship between microbiota composition in the digestive tract and FE remain to be confirmed in more and larger scale studies. Results of the present experiment suggest that there are possibilities to modify the intestinal microbial composition by means of nutrition (e.g., by use of specific additives such as pro- and prebiotics) in order to improve FE of grower-finisher pigs. 



\section{4}

\section{Prediction of nutrient digestibility in grower- finisher pigs based on faecal microbiota composition}

L.M.G. Verschuren ${ }^{1,2}$, D. Schokker ${ }^{2}$, R. Bergsma ${ }^{1}$, A.J.M. Jansman ${ }^{2}$, F. Molist ${ }^{3}$, M.P.L. Calus ${ }^{4}$

${ }^{1}$ Topigs Norsvin Research Center B.V., P.O. Box 43, 6640 AA Beuningen, The Netherlands; ${ }^{2}$ Wageningen University and Research, Wageningen Livestock Research, P.O. Box 338, 6700 AH Wageningen, Netherlands; ${ }^{3}$ Schothorst Feed Research B.V., P.O. Box 533, 8200 AM Lelystad, The Netherlands; ${ }^{4}$ Wageningen University and Research, Animal Breeding and Genomics, P.O. Box 338, $6700 \mathrm{AH}$ Wageningen, Netherlands

Journal of animal breeding and genetics (2020) 1:23-35 


\begin{abstract}
Microbiota play an important role in total tract nutrient digestion, especially when fibrous diets are fed to pigs. This study aimed to use metagenomics to predict faecal nutrient digestibility in grower-finisher pigs. The study design consisted of 160 threeway crossbreed grower-finisher pigs ( 80 female and 80 male) which were either fed a diet based on corn/soybean meal or a more fibrous diet based on wheat/barley/byproducts. On the day before slaughter, faecal samples were collected and used to determine faecal digestibility of dry matter, ash, organic matter, crude protein, crude fat, crude fibre and non-starch polysaccharides. The faecal samples were also sequenced for the $16 \mathrm{~S}$ hypervariable region of bacteria (V3/V4) to profile the faecal microbiome. With these data we calculated the between animal variation in faecal nutrient digestibility associated with variation in the faecal microbiome, that is the "microbiability". The microbiability values were significantly greater than zero for dry matter, organic matter, crude protein, crude fibre and non-starch polysaccharides, ranging from 0.58 to 0.93 , as well as for crude fat with a value of 0.37 , but not significantly different from zero for ash. Using leave-one-out cross-validation, we estimated the accuracy of predicting digestibility values of individual pigs based on their faecal microbiota composition. The accuracies of prediction for crude fat and ash digestibility were virtually 0 , and for the other nutrients the accuracies ranged from 0.42 to 0.63 . In conclusion, the faecal microbiota composition gave high microbiability values for faecal digestibility of dry matter, organic matter, crude protein, crude fibre, and non-starch polysaccharides. The accuracies of prediction are relatively low if the interest is in precisely predicting faecal nutrient digestibility of individual pigs, but are promising from the perspective of ranking animals in a genetic selection context.
\end{abstract}

Keywords: Digestion, microbiota, pig, metagenomic 


\subsection{Introduction}

Feed efficiency is important in the pork industry, as feed costs comprise the main cost of production. Feed efficiency is a complex trait; within diet-variation in feed efficiency between pigs is phenotypically associated with feeding behaviour (Shirali et al., 2017), adipose, muscle and/or liver tissue gene expression (Horodyska et al., 2018a; Horodyska et al., 2019a; Horodyska et al., 2019b) and faecal microbiota composition (Vigors et al., 2016a; Camarinha-Silva et al., 2017; Yang et al., 2017; Tan et al., 2018; Verschuren et al., 2018) . Another trait associated with variation in feed efficiency is faecal nutrient digestibility, as divergent selection for feed efficiency in a selection experiment resulted in lines of pigs showing differences in faecal nutrient digestibility values (Harris et al., 2012; Mauch et al., 2018). The nutrients which are not digested by the pigs' digestive enzymes are in part fermented by gut microbiota residing in the gastrointestinal tract. Using metagenomics, which is the genomic analysis of microorganisms (Handelsman, 2004), it has been shown that gut microbiota contribute to within-diet variation in faecal digestibility of nutrients in pigs, as faecal microbial operational taxonomic units (OTUs), phyla and genera have been correlated with digestibility of energy, crude protein (CP) and cell wall components (Niu et al., 2015; Le Sciellour et al., 2018). Focus on specific microbial OTUs, phyla or genera related to variation in faecal nutrient digestibility, however, ignores the complexity of the microbial coherence and interrelationship. Using a whole community approach, the percentage of phenotypic variation that is associated with differences in faecal microbiota composition, which has been termed microbiability (Difford et al., 2016), can be estimated. This approach has successfully been used to associate faecal microbiota composition with complex traits in pigs (Camarinha-Silva et al., 2017), chickens (Wen et al., 2019), cattle (Difford et al., 2016) and humans (Ross et al., 2013). Using faecal microbiota composition to predict faecal nutrient digestibility could be an alternative to traditional methods to measure nutrient digestibility, which are more expensive to use on the large scale that is necessary for breeding.

This study investigated the use of metagenomics in grower-finisher pigs to identify the proportion of phenotypic variance associated with and the accuracy of prediction of faecal nutrient digestibility values by faecal microbiota composition. We considered two diets that are representative for the main diets fed to pigs in commercial and breeding herds across the world. 


\subsection{Material and methods}

This study was carried out in strict accordance with the recommendations in the European Guidelines for accommodation and care of animals. The protocol was approved by the Animal Care and Use Committee of Schothorst Feed Research B.V, Lelystad, The Netherlands (Protocol Number: AVD 246002015120/132) and is in strict accordance with Directive 2010/63/EU.

\subsubsection{Animals and experimental design}

Pigs used in this study originated from a three-way cross, that is Synthetic boar $x$ (Large White $x$ Landrace) sow. Phenotypic data were available for 160 pigs, 80 males and 80 females, coming from 21 litters. All pigs were kept under commercial conditions at the experimental facilities of Schothorst Feed Research B.V. Before the start of the study, pigs were housed per litter, with $22.5 \%$ of the pigs being crossfostered, and all pigs were fed the same diet. The pigs entered the study at 59-67 days of age (day 0 ), in two groups of 80 , and experimental groups were set 20 days apart. Ten pigs were housed per pen and eight pens per compartment. One compartment was used per entrance date. Littermates were randomly distributed over the two diets and males and females were housed in separate pens, resulting in two pens per diet per sex per entrance date. At the start of the experiment, the pigs had an average BW of $22.3 \mathrm{~kg}$ and were kept in the facilities until they reached a live weight at slaughter of approximately $120 \mathrm{~kg}$ (mean age 168 days). Pigs were allowed a minimal space of $1 \mathrm{~m}^{2}$ per pig, and the pens had a concrete floor for $60 \%$ and a slatted floor for $40 \%$.

\subsubsection{Feeding strategy}

Two diets were studied, a diet based on corn/soybean meal (CS) as typically fed to commercial grower-finisher pigs and pig breeding herds in The Americas and a more fibrous diet based on wheat/barley/by-products (WB) as typically fed to pigs in Europe. The pigs were fed ad libitum according to a three-phase feeding program. The pigs were fed a starter diet from day 0 to day 25, a grower diet from day 26 to day 67 and a finisher diet from day 68 until they reached slaughter weight. The diets were formulated on a fixed ratio of standardized ileal (SID) lysine to net energy (NE). Diets in each of the three phases had a different SID lysine to NE ratio, being 1.12 $\mathrm{g} / \mathrm{MJ}$ in the starter diet, $0.94 \mathrm{~g} / \mathrm{MJ}$ in the grower diet and $0.73 \mathrm{~g} / \mathrm{MJ}$ in the finisher diet. The decrease of SID lysine to NE ratio in grower and finisher diets was mainly achieved by exchanging soybean meal with corn for the CS diet, and peas with wheat for the WB diets. A premix was added to the finisher diet; this premix contained 
titanium dioxide as digestibility marker $(0.25 \%$ at the level of the diets). The experimental diets were produced by ABZ Diervoeding, Leusden, The Netherlands.

\subsubsection{Measurements and sampling}

The experimental facilities (Schothorst Feed Research B.V.) were equipped with IVOG feeding stations (INSENTEC) that register individual feed intake of group housed pigs. All pigs had ear tags with unique incremental numbering; therefore, individual feed intake records were available for all pigs for each day on test. Pigs were weighted at day 0 , day 56 and at the end of the study. One day before slaughter, individual faecal grab samples were collected immediately at defecation. Faecal samples destined for microbiota analysis were collected of 142 out of 160 pigs, due to death (6) and insufficient sample volume (12), immediately frozen in liquid nitrogen and stored at $-80^{\circ} \mathrm{C}$. Due to sample volume needed for the chemical analyses, faecal samples destined for nutrient analysis for digestibility determination were collected of 105 pigs, stored at $4{ }^{\circ} \mathrm{C}$ and, freeze-dried, and dry samples were milled over a $1 \mathrm{~mm}$ sieve prior to chemical analyses. Diets and faecal samples were analysed in duplicate for moisture, ash, starch, crude protein, crude fat (CFat), crude fibre (CF) and titanium oxide marker using the following methods: ISO 6496, NEN 3329, NEN-ISO 15914:2005 en, ISO/CD 15670, ISO/FDIS 6492 method B, ISO6865:2001 and EEG 26-11-1992 nr.L344/35-37 (Method based on Short et al. (1996)) respectively. Dry matter (DM) ( $\mathrm{g} / \mathrm{kg}$ as is) was calculated as:

1 - moisture ( $\mathrm{g} / \mathrm{kg}$ as is),

Organic matter (OM) (g/kg DM) was calculated as:

$D M(g / k g$ as is) - Ash ( $g / k g D M)$,

and the non-starch polysaccharides (NSP) fraction (g/kg DM) was calculated as:

$D M(g / k g$ as is $)-(A s h(g / k g D M)+\operatorname{starch}(g / k g D M)+C P(g / k g D M)+$ CFat $(g / k g D M))$.

Faecal nutrient digestibility values were calculated in percentages based on concentrations of the marker and the nutrient in the diet and faeces as:

$[1$

$\left.-\left(\frac{\text { conc. of marker in the diet }(\mathrm{mg} / \mathrm{kg} D M)}{\text { conc. of marker in the faeces }(\mathrm{mg} / \mathrm{kg} D M)}\right)\left(\frac{\text { conc. of nutrient in the faeces }(\mathrm{g} / \mathrm{kg} D M)}{\text { conc. of nutrient in the } \operatorname{diet}(\mathrm{g} / \mathrm{kg} D M)}\right)\right]$ $\times 100 \%$ 


\section{Faecal microbiota and nutrient digestion in pigs}

\subsubsection{Faecal microbiota analysis}

For each sample, the faecal microbiome was profiled by sequencing the $16 \mathrm{~S}$ hypervariable region of bacteria. For microbial DNA extraction a standardized protocol was used and an optical density measurement to check the quality was performed on the Nanodrop (Agilent Technologies). PCR was used to amplify the $16 \mathrm{~S}$ rDNA V3/V4 fragment using forward primer V3_F (CCTACGGGAGGCAGCAG) and reverse primer V4_R (GGACTACHVGGGTWTCT). Whereby the following PCR conditions were used: $2 \mathrm{~min}$ at $98^{\circ} \mathrm{C}, 15 \times\left(10 \mathrm{~s}\right.$ at $98^{\circ} \mathrm{C}, 30 \mathrm{~s}$ at $55^{\circ} \mathrm{C}, 10 \mathrm{~s}$ at $\left.72^{\circ} \mathrm{C}\right), 7$ min at $72^{\circ} \mathrm{C}$. PCR efficiency was checked on agarose gel by visual inspection. Subsequently samples were sequenced by targeted-amplicon $16 \mathrm{~S}$ sequencing using the MiSeq sequencer (Illumina) and analysed for taxonomy profile per sample with clustering by profile using the open-source software pipeline QIIME (Caporaso et al., 2010). Standard assembly based on amplicon, with primer removal was performed. For Quality filtration of the sequences the following settings were used: (a) Phred $>$ Q20 and (b) amplicons >100 bases. For the data analysis, pseudoreads were clustered into OTUs per sample at $97 \%$ similarity and chimeras were removed with Chimeraslayer (Haas et al., 2011). To get taxonomic information, sequences representative for every OTU were aligned against the Greengenes core set (13_8 release) (DeSantis et al., 2006; McDonald et al., 2012).

To investigate the difference in microbiota biodiversity of the faecal samples between the diets, sexes and litters (after cross-fostering), we calculated the Shannon diversity index and the Chao richness index based on the OTU count data for each sample using R package "vegan" (Oksanen et al., 2017). Significance of differences in the diversity estimates between the diets, sexes and litters was determined using a linear model with diet, sex and litter modelled as fixed effects. The R package "car" (Fox and Weisberg, 2011) was used for estimating the $P$-values, and least squares means were computed using the R package "Ismeans" (Lenth, 2016). The dissimilarity between the samples in their microbiota composition was investigated by calculating the Bray-Curtis distances based on OTU count data using R package "vegan" (Oksanen et al., 2017). The same R package was used to test for significance of differences in Bray-Curtis distances between diets, sexes and litters, by means of a permutational multivariate analysis of variance using distance matrices (ADONIS) with a maximum of 999 permutations (Oksanen et al., 2017). All previously described analyses were based on the entire OTU count data. For the following steps, relative abundance of OTUs was calculated and OTUs with average relative abundance smaller than $0.001 \%$ and present in $<5 \%$ of the animals were discarded. The OTU table of relative abundance was analysed by discriminant 
analysis using principal components (DAPC) (Jombart et al., 2010), to test the association of OTU relative abundance with diet, sex and litter, as described previously (Verschuren et al., 2018).

\subsubsection{Phenotypic prediction using microbiota data}

Nutrient digestibility was predicted based on microbial relationship matrices (Ross et al., 2013). This approach uses a model commonly used in breeding and genetics to predict breeding values based on pedigree or genomic relationships. In short, the metagenomic profiles (matrix $\mathbf{X}[n \times m]$ ), with $n$ samples and $m$ OTUs, were defined based on the relative abundance of OTUs. Considering relative abundance $R A_{i j}$ for sample $i$ and taxonomic unit $j, X_{i j}=\log \left(R A_{i j}-\overline{R A}_{. j}\right)$ (Ross et al., 2013). Subsequently, a metagenomic relation matrix was computed based on these metagenomic profiles as: $\mathbf{M}=\mathbf{X} \mathbf{X}^{\prime} / m$.

The following mixed model was used for the estimation of the microbiability and the proportion of the variance explained by the common litter effect:

$\mathbf{y}=\mathbf{X b}+\mathbf{Z m}+\mathbf{U c}+\mathbf{e}$,

where $\mathbf{y}$ is the vector of phenotypes (one record per sample), $\mathbf{X}$ is the incidence matrix for the fixed effects for sex, diet and pen, $\mathbf{b}$ are the fixed effects, $\mathbf{Z}$ the incidence matrix for OTU effects, $\mathbf{m}$ the random effect estimate of OTU $\sim N\left(\mathbf{0}, \mathbf{M} \sigma^{2} \mathrm{~m}\right)$, $\mathbf{U}$ the incidence matrix for common litter effect (foster dam in case of crossfostering), $\mathbf{c}$ the random effect estimate of common litter $\sim N\left(\mathbf{0}, \mathbf{I} \sigma^{2}{ }_{c}\right)$ and $\mathbf{e}$ the random residuals estimate $\sim N\left(\mathbf{0}, \mathbf{I} \sigma^{2}{ }_{\mathrm{e}}\right)$. ASReml (Gilmour et al., 2015) was used to simultaneously estimate $\boldsymbol{\sigma}^{2}{ }_{m}, \boldsymbol{\sigma}^{2}{ }_{c}, \widehat{\mathbf{m}}$ and $\hat{\boldsymbol{c}}$ from the following equation:

$\left[\begin{array}{c}\hat{\mathbf{b}} \\ \widehat{\mathbf{m}} \\ \hat{\boldsymbol{c}}\end{array}\right]=\left[\begin{array}{ccc}\mathbf{X}^{\prime} \mathbf{X} & \mathbf{X}^{\prime} \mathbf{Z} & \mathbf{X}^{\prime} \mathbf{U} \\ \mathbf{Z}^{\prime} \mathbf{X} & \mathbf{Z}^{\prime} \mathbf{Z}+\mathbf{M}^{-1} \frac{\boldsymbol{\sigma}^{2}{ }_{e}}{\boldsymbol{\sigma}^{2} m} & \mathbf{Z}^{\prime} \mathbf{U} \\ \mathbf{U}^{\prime} \mathbf{X} & \mathbf{U}^{\prime} \mathbf{Z} & \mathbf{U}^{\prime} \mathbf{U}+\mathbf{I} \frac{\boldsymbol{\sigma}^{2}{ }_{e}}{\boldsymbol{\sigma}^{2}{ }_{c}}\end{array}\right]^{-1}\left[\begin{array}{l}\mathbf{X}^{\prime} \mathbf{y} \\ \mathbf{Z}^{\prime} \mathbf{y} \\ \mathbf{U}^{\prime} \mathbf{y}\end{array}\right]$

Solving the equations results in estimates for the fixed effects $(\hat{\mathbf{b}})$ as well as the effect for each metagenomic profile, such that $\widehat{\mathbf{m}}$ has the dimensions $n \times 1$. The microbiability was computed as $\widehat{m}^{2}=\hat{\sigma}_{m}^{2} /\left(\hat{\sigma}_{m}^{2}+\hat{\sigma}_{c}^{2}+\hat{\sigma}_{e}^{2}\right)$ (Difford et al., 2016) and the proportion of variance due to common litter effect as $\hat{c}^{2}=\hat{\sigma}_{c}^{2} /\left(\hat{\sigma}_{m}^{2}+\hat{\sigma}_{c}^{2}+\hat{\sigma}_{e}^{2}\right)$. To investigate whether effects on digestibility of metagenomic profiles and common litter were related, the model was additionally put to run including only the metagenomic profiles:

$\mathbf{y}=\mathbf{X b}+\mathbf{Z m}+\mathbf{e}$,

or only common litter:

$y=X b+U c+e$, 
where $\mathbf{y}, \mathbf{X}, \mathbf{b}, \mathbf{Z}, \mathbf{m}, \mathbf{U}, \mathbf{c}$ and $\mathbf{e}$ are as described for model 1. Significance of each of the random effects was tested by means of the log-likelihood ratio test, using the test statistic $D=2\left[\log \left(L_{2}\right)-\log \left(L_{1}\right)\right]$, where $L_{2}$ is the likelihood of the model including a specific random effect and $L_{1}$ the likelihood of the model excluding this random effect, as provided by ASReml (Gilmour et al., 2015). This means that model 1 was compared with model 2 to test the significance of the common litter effect, and with model 3 to test the significance of the random effect of the metagenomic profiles. Likewise, both models 2 and 3 were compared to model 4 that included no random effect:

$\mathbf{y}=\mathbf{X b}+\mathbf{e}$,

where $\mathbf{y}, \mathbf{x}, \mathbf{b}$ and $\mathbf{e}$ are as described for model 1 . The distribution of the $\mathrm{D}$ test statistics is a mixture of two chi-square distributions with 0 and 1 degrees of freedom (Self and Liang, 1987). Considering this, the $D$ test statistics were used to calculate $P$ values, where values $<0.05$ were considered to be significant.

To assess the accuracy of the metagenomic predictions, the residuals from the above specified model 3 were used as precorrected phenotypes $\mathbf{y}^{*}$ (corrected for fixed effects sex, diet and pen, and for the random common litter effects) in combination with leave-one-out cross-validation. In each of the validation folds, the precorrected phenotype of one of the individuals was removed, and predicted using the remaining individuals. In this way, a metagenomic prediction for each of the individuals was obtained, based on the digestibility values of all other animals in the data and all metagenomic profiles. The model used for the leave-one-out cross-validation was:

$\mathbf{y}^{*}=\mathbf{1}_{\mathrm{n}} \mu+\mathbf{Z m}+\mathbf{e}$,

where $\mathbf{1}_{n}$, is a vector of ones; $\boldsymbol{\mu}$ is the overall mean; $\mathbf{Z}, \mathbf{m}$ and $\mathbf{e}$ are as described in model 1 ; and for each individual, the predicted precorrected phenotype obtained from model (5) is: $\hat{y}^{*}=\hat{\mu}+\widehat{m}_{i}$. The accuracy of the predictions was obtained by computing the correlation between observed precorrected phenotypes $\mathbf{y}^{*}$ and metagenomic predictions $\widehat{\mathbf{m}}$ of the nutrient digestibility values obtained from model (5). In addition, $\mathbf{y}^{*}$ was regressed on $\hat{\mathbf{m}}$ to evaluate bias of the predictions. Whether or not the accuracy was significantly different from 0 , was assessed by obtaining the distribution of the accuracy using 10,000 bootstrap samples to recompute the accuracy.

\subsection{Results}

An overview of the faecal microbiota composition per diet and sex is given in Figure 4.1, and an overview per litter is provided in Figure 4.2. The considerable differences 
in faecal microbiota composition between diets, sexes and litters shown in Figure 4.1 and 4.2 were also visible in the microbial biodiversity indicators (Table 4.1). The Shannon diversity index was significantly higher for the pigs fed the CS diet and lowest for boars, whereas the Chao richness index was not significantly different between the diets and sexes. Shannon diversity index and the Chao richness index indicated significant differences between litters. The Bray-Curtis distances indicated that the microbiota composition of samples was significantly different for diets $(p=$ $.001)$, sexes $(p=.001)$ and litters $(p=.001)$. The faecal microbiota composition, measured in relative abundance, was significantly associated with diet and $\operatorname{sex}(p=$ .011) when using two discriminators and 15 principle components describing $83 \%$ of the variation in microbiota composition (Figure 4.3). The DAPC analysis could also distinguish 5 litters from the other 21 litters $(p<.05)$ based on the faecal microbiota composition when using 3 discriminators and 30 principle components, which captured $92 \%$ of the variation. The biodiversity and microbiota composition analyses indicate that the faecal microbiota was influenced by diet, sex and litter.
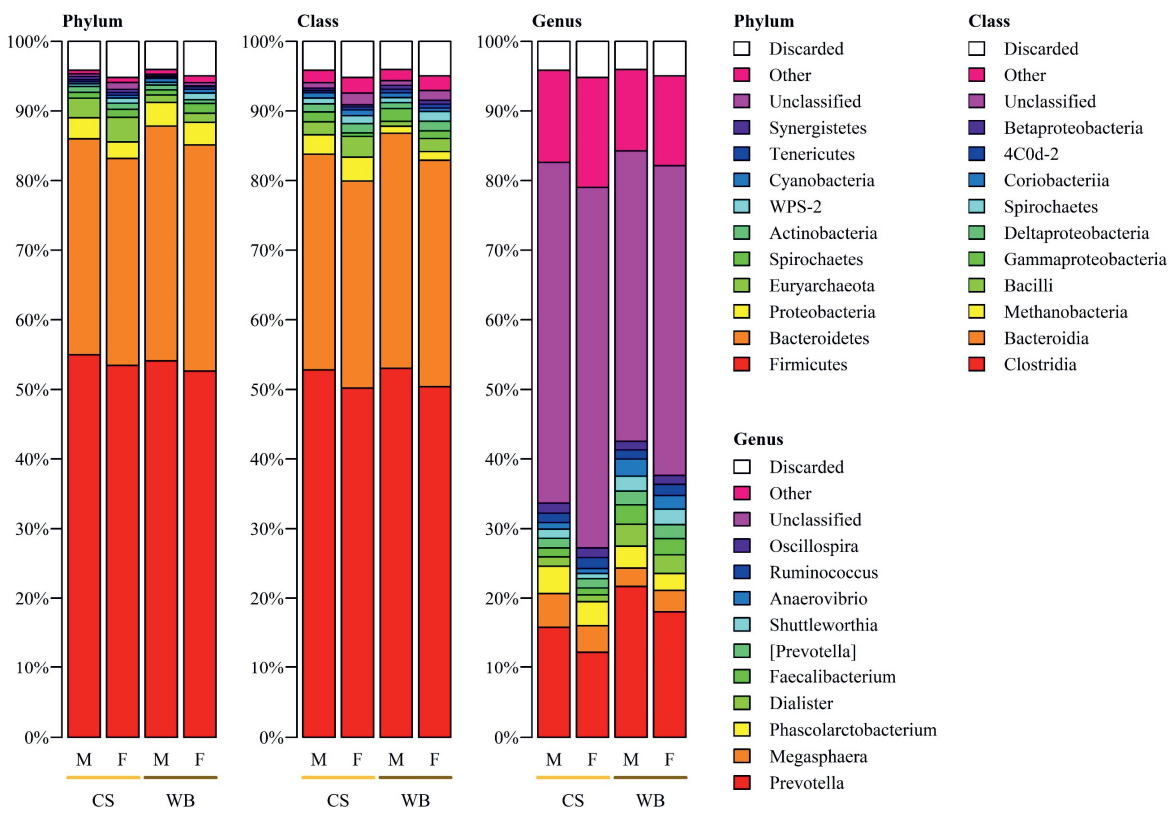

Figure 4.1 Relative abundance of 10 major bacterial phyla, classes and genera in the faeces of male (M) and female (F) pigs fed a corn/soybean meal diet (CS) or a wheat/barley/by-products diet (WB). Data are mean percentage of total identified sequences. 

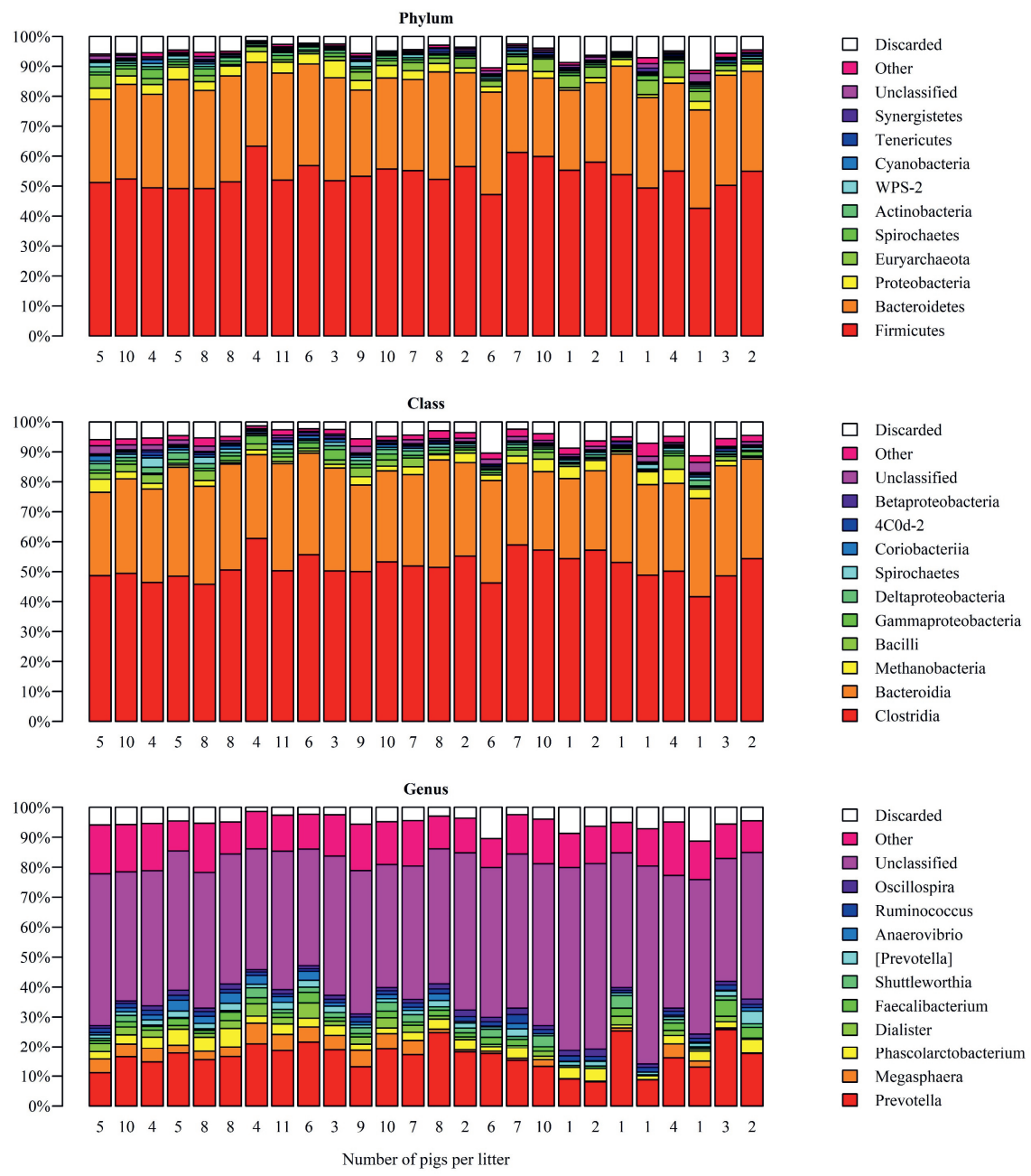

Figure 4.2 Relative abundance of 10 major bacterial phyla, classes and genera in the faeces of pigs raised in a common litter. Data are mean percentage of total identified sequences and every bar represents the means of one litter.

Table 4.1 Overview of faecal microbial biodiversity across animals per experimental diet and sex.

\begin{tabular}{|c|c|c|c|c|c|c|c|}
\hline \multirow{2}{*}{ Index } & \multicolumn{3}{|c|}{ Diet } & \multicolumn{3}{|c|}{ Sex } & \multirow{2}{*}{$\begin{array}{c}\text { Litter } \\
\text { P-value }\end{array}$} \\
\hline & $\mathrm{CS}^{\mathrm{a}}$ & $W^{a}$ & P-value & Boars $^{a}$ & Gilts $^{a}$ & P-value & \\
\hline diversity & 5.99 & 5.84 & 0.015 & 5.82 & 6.01 & 0.002 & 0.001 \\
\hline Chao richness & 19925 & 19098 & 0.447 & 18587 & 20436 & 0.085 & $<0.001$ \\
\hline
\end{tabular}

Abbreviations: CS, corn/soybean meal diet; WB, wheat/barley/by-products diet.

a Values are least squares means. 


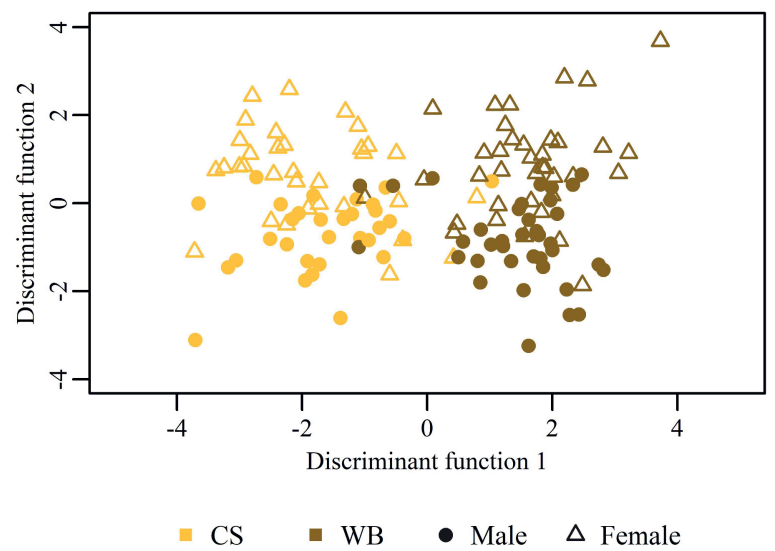

Figure 4.3 Gaussian kernel density estimation of the discriminant function as result of the discriminant analysis of principle components for male (circle) and female (triangle) pigs fed a corn/soybean meal diet (yellow) or a wheat/barley/by-products diet (brown).

Average faecal nutrient digestibility values ranged from $25.7 \%$ for NSP of boars fed the CS diet to $88.3 \%$ for OM of gilts fed the CS diet (Table 4.2). Variation in faecal nutrient digestibility between pigs was lowest for OM of gilts fed the CS diet (SD = $1.1 \%$ ), whereas CF of boars fed the WB diet had the highest variation ( $S D=8.7 \%$ ). $p$ Values of the fixed effects sex, diet and pen in all three of the models are presented in Table 4.3. Diet significantly influenced the faecal digestibility values of all nutrients in all models, whereas pen did not affect digestibility values of any of the nutrients in any of the models. In all models, sex significantly influenced faecal nutrient digestibility values of DM, OM, CP and CF. Sex did not affect faecal nutrient digestibility values of CFat and Ash in any of the three models, and for NSP, the effect of sex in the model tended to be significant.

The estimates of $m^{2}$ and $c^{2}$ of all three models are presented in Table 4.4. When using the model including both microbiota and common litter information (model 1 ), $\mathrm{m}^{2}$ ranged from 0.013 for ash to 0.932 for CP. However, the SE of the $m^{2}$ estimate of ash was higher than the $m^{2}$ estimate itself and was not significantly different from zero $(p=.438)$. For CFat the SE (0.24) was also high compared to $m^{2}(0.37)$. Using the same model including both microbiota and common litter information (model 1), $c^{2}$ estimates were zero for all of the nutrients except for CFat and CF, which had estimates of 0.12 and 0.02 , respectively and were not significantly different from zero. Comparing the results of the model including both metagenomic profile and common litter (model 1 ) with those of the models including only one of the 


\section{Faecal microbiota and nutrient digestion in pigs}

Table 4.2 Overview of (variation in) faecal nutrient digestibility (\%) across animals per experimental diet and sex

\begin{tabular}{|c|c|c|c|c|c|c|c|c|c|c|c|c|}
\hline \multirow[b]{3}{*}{ Nutrient } & \multicolumn{6}{|c|}{ Corn/soybean meal diet } & \multicolumn{6}{|c|}{ Wheat/barley/by-products diet } \\
\hline & \multicolumn{3}{|c|}{ Boars } & \multicolumn{3}{|c|}{ Gilts } & \multicolumn{3}{|c|}{ Boars } & \multicolumn{3}{|c|}{ Gilts } \\
\hline & $\mu$ & $\sigma$ & \# & $\mu$ & $\sigma$ & \# & $\mu$ & $\sigma$ & \# & $\mu$ & $\sigma$ & \# \\
\hline Dry Matter & 86.8 & 1.3 & 20 & 87.4 & 1.2 & 29 & 73.3 & 2.3 & 25 & 75.4 & 1.9 & 31 \\
\hline Ash & 58.6 & 3.3 & 20 & 56.8 & 3.0 & 29 & 42.3 & 3.0 & 24 & 43.0 & 3.0 & 31 \\
\hline $\begin{array}{l}\text { Organic } \\
\text { Matter }\end{array}$ & 87.6 & 1.4 & 20 & 88.3 & 1.1 & 29 & 74.4 & 2.5 & 24 & 76.5 & 2.0 & 31 \\
\hline $\begin{array}{l}\text { Crude } \\
\text { Protein }\end{array}$ & 80.4 & 3.2 & 20 & 81.8 & 2.1 & 29 & 67.1 & 3.1 & 25 & 69.7 & 3.4 & 31 \\
\hline Crude Fat & 81.3 & 1.7 & 19 & 80.9 & 2.0 & 29 & 76.4 & 2.3 & 24 & 77.0 & 1.9 & 31 \\
\hline Crude Fibre & 49.9 & 5.5 & 20 & 54.4 & 5.9 & 29 & 29.4 & 8.7 & 25 & 38.2 & 6.6 & 31 \\
\hline NSP & 25.7 & 8.0 & 19 & 29.3 & 7.6 & 29 & 41.3 & 6.3 & 24 & 46.7 & 4.8 & 31 \\
\hline
\end{tabular}

Abbreviations: \#, number of records; $\mu$, average; NSP, non-starch polysaccharides; $\sigma$, standard deviation.

Table 4.3 $p$-Values for the effect of sex, diet and pen on faecal nutrient digestibility with a model including microbiability and common litter effect $\left(m^{2}+c^{2}\right)$, and the models only including microbiability $\left(\mathrm{m}^{2}\right)$ or common litter effect $\left(c^{2}\right)$

\begin{tabular}{|c|c|c|c|c|c|c|c|c|c|}
\hline \multirow[b]{2}{*}{ Nutrient } & \multicolumn{3}{|c|}{$m^{2}+c^{2}$} & \multicolumn{3}{|l|}{$m^{2}$} & \multicolumn{3}{|l|}{$c^{2}$} \\
\hline & Sex & Diet & Pen & Sex & Diet & Pen & Sex & Diet & Pen \\
\hline Dry Matter & .001 & $<.001$ & .194 & .001 & $<.001$ & .193 & $<.001$ & $<.001$ & .576 \\
\hline Ash & .642 & $<.001$ & .280 & .713 & $<.001$ & .247 & .825 & $<.001$ & .281 \\
\hline Organic Matter & .002 & $<.001$ & .285 & .002 & $<.001$ & .285 & $<.001$ & $<.001$ & .762 \\
\hline Crude Protein & .003 & $<.001$ & .435 & .003 & $<.001$ & .435 & $<.001$ & $<.001$ & .817 \\
\hline Crude Fat & .331 & $<.001$ & .268 & .262 & $<.001$ & .157 & .572 & $<.001$ & .357 \\
\hline Crude Fibre & $<.001$ & $<.001$ & .251 & $<.001$ & $<.001$ & .251 & $<.001$ & $<.001$ & .474 \\
\hline NSP & .054 & $<.001$ & .241 & .054 & $<.001$ & .241 & .004 & $<.001$ & .254 \\
\hline
\end{tabular}

$p$-values below 0.05 indicate significant effect.

Abbreviations: NSP, non-starch polysaccharides.

information sources (model 2 and 3 ) did not change the overall result. Therefore, in this data set, there was no evidence that faecal nutrient digestibility of litter mates was similar due to encountering a common environment in their early life, including being nursed by the same sow. In contrast, the $m^{2}$ estimates of DM, OM, CP, CF and NSP were clearly significantly greater than zero, showing that the faecal microbiota composition is highly associated with the digestibility of these nutrients. The maximum individual OTU contribution to the estimated $\mathrm{m}^{2}$ of faecal CP digestibility was $1.08 \%$ and the top 25 OTUs contributed $10 \%$ altogether (Table 4.5 ). All individual OTU contributions to the prediction of faecal nutrient digestibility values are provided in Table 4.S1. 


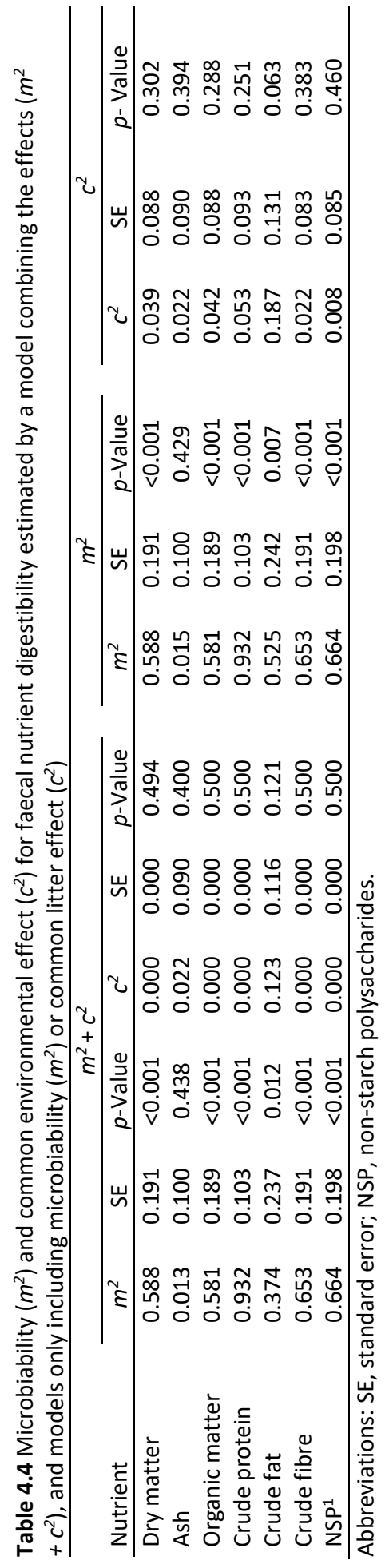




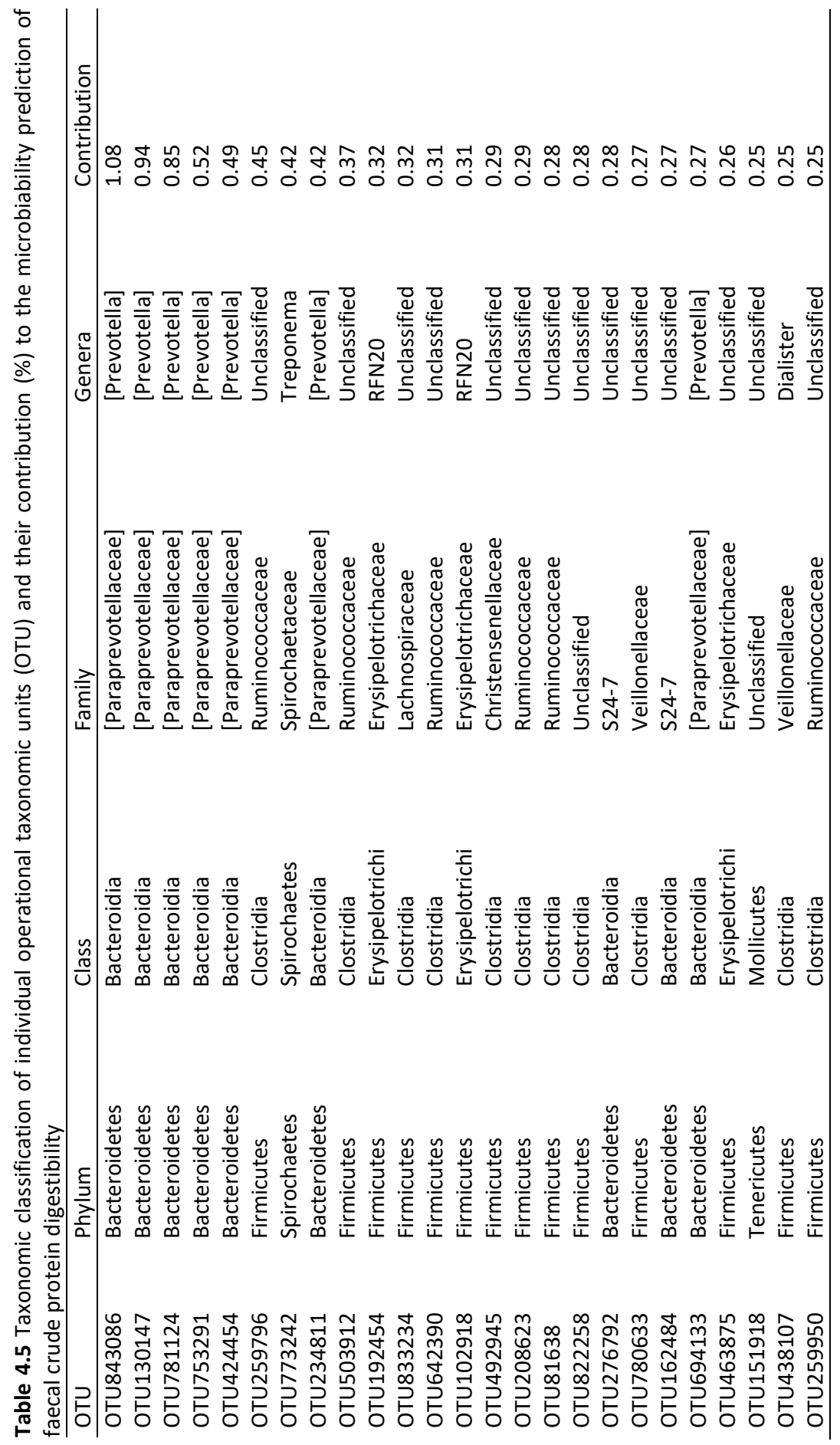


The results of the predictions of faecal nutrient digestibility based on faecal metagenomic profiles are presented in Table 4.6. The prediction generated accuracies up to 0.63 for $\mathrm{CP}$. Based on the $95 \%$ confidence interval, the prediction accuracy of ash and CFat were not significantly different from zero. Despite the apparent significance of the $m^{2}$ for CFat $(p=.012)$, both the standard error of the estimate and the accuracy of prediction suggest that variation in digestibility of CFat is hardly related to variation in faecal microbiota composition. The accuracies of prediction for faecal digestibility of all other nutrients (DM, OM, CF and NSP) ranged between 0.3 and 0.5 , and were significantly different from zero. The intercepts of the regression of observed on predicted digestibilities for DM, OM, CP, CF and NSP were all close to zero, while the regression coefficients were close to one, indicating that the predicted values were unbiased.

Table 4.6 Leave-one-out cross-validation results for predicted faecal nutrient digestibility

\begin{tabular}{llllll} 
Nutrient & Accuracy & $\mathrm{L}-\mathrm{Cl}$ & $\mathrm{U}-\mathrm{Cl}$ & Intercept & Slope \\
\hline Dry matter & 0.42 & 0.28 & 0.55 & 0.00 & 1.06 \\
Ash & -0.76 & -0.99 & -0.68 & 0.04 & -38 \\
Organic matter & 0.43 & 0.29 & 0.56 & 0.00 & 1.08 \\
Crude protein & 0.63 & 0.52 & 0.72 & 0.01 & 1.04 \\
Crude fat & 0.01 & -0.15 & 0.18 & 0.00 & 0.07 \\
Crude fibre & 0.42 & 0.29 & 0.55 & -0.01 & 1.04 \\
NSP & 0.43 & 0.31 & 0.55 & -0.03 & 1.06 \\
\hline
\end{tabular}

Abbreviations: Accuracy, accuracy of prediction; Intercept, intercept of the regression of observed on predicted digestibility; L-Cl, lower bound of the confidence interval; NSP, nonstarch polysaccharide; Slope, slope of the regression of observed on predicted digestibility values; $\mathrm{U}-\mathrm{CL}$, upper bound of the confidence interval.

\subsection{Discussion}

\subsubsection{Metagenomics}

This study investigated the use of metagenomics to identify the proportion of variance in faecal nutrient digestibility values associated with faecal microbiota composition in pigs. Faecal microbiota composition and diversity was affected by diet, sex, and litter, whereas faecal nutrient digestibility was affected by diet and sex, but not by litter. Despite the effects of diet, sex and litter, we found that the faecal digestibility of DM, OM, CP, CF, and NSP in grower-finisher pigs is associated with faecal microbiota composition, whereas no such association was observed for ash and CFat digestibility. Camarinha-Silva et al. (2017) were the first to estimate microbiability for complex traits in pigs and found values of $0.28,0.16$, and 0.21 for average daily gain, feed intake and feed conversion rate respectively, which are lower than our $m^{2}$ estimates for faecal nutrient digestibility. Previous research found 


\section{Faecal microbiota and nutrient digestion in pigs}

both positive and negative correlations between relative abundance of individual OTU and faecal digestibility of energy, CP and cell wall components as well, but only when pigs were fed a low fibre diet (Le Sciellour et al., 2018). The strongest correlation, between faecal digestibility of CP and an OTU classified as Clostridium, was -0.45 (Le Sciellour et al., 2018). A correlation of -0.45 suggest that the proportion of variance explained by this single OTU was 0.20 , whereas we found a microbiability of 0.93 for CP. Niu et al. (2015) did not find a correlation between specific microbiota (phyla or genera) and faecal nutrient digestibility of CP in pigs. They did find a significant correlation with faecal nutrient digestibility of CF, NDF, ADF and hemicellulose, however, with the highest correlations being $0.61,0.55,0.65$ and 0.45, respectively (Niu et al., 2015). These correlations correspond with explained proportions of variances of 0.20 to 0.37 , which are lower but in the same order of magnitude as our estimates of microbiability for CF (0.65) and NSP (0.66). Our estimated contributions of individual OTU to the predictions are low, with the most important OTU contributing only $1.08 \%$ to $\mathrm{m}^{2}$ of faecal CP digestibility. We used OTUs instead of a taxonomic rank for our predictions, which provided us with more information on the whole faecal microbial community, however, at the cost of taxonomic and functionality knowledge. Nevertheless, the OTUs contributing most to the $\mathrm{m}^{2}$ of faecal CP digestibility belonged to the genus Prevotella, which was one of the most abundant genera in our samples. The limited power to estimate individual OTU effects was due to the size of the dataset. This limitation was overcome by including all microbiota simultaneously in the model, where modelling a variance component for microbiota implied that estimates of individual OTU effects were subject to shrinkage. Therefore, the lower association between faecal microbiota composition and faecal nutrient digestibility in literature compared to the relatively high microbiability values found in our study are likely due to the focus on specific microbiota compared to our focus on the whole community.

Pigs originating from the same litter may have similar performance both due to being genetically related, and due to sharing a common environment during part of their life. This common environmental effect was modelled as a common litter effect. We found no effect of common litter on faecal digestibility of any of the nutrients. Ouweltjes et al. (2018) showed that common litter contributes up to $7.8 \%$ of the phenotypic variance of faecal digestibility of nutrients. The common litter effects measured in our study, although not significantly different from zero, are in the same order of magnitude. Arguably, common litter effects can be due to factors affecting gastrointestinal development during both the pre- and postnatal period, which might influence faecal digestibility of nutrients later in life. Before parturition, the 
intrauterine environment affects the growth and development of the gastrointestinal tract (Sangild et al., 2000). After birth, the colostrum and milk composition greatly shape the growth, development and functioning of the gastrointestinal tract (Pluske, 2016). Microbiota affect intestinal functioning and development after parturition as well, as early life microbial colonization in pigs modulate the intestinal immune system (Weng and Walker, 2013), morphology, digestive enzyme activity (Arnal et al., 2014), and gene expression (Schokker et al., 2015) at later ages. Even though early-life colonization in pigs is affected by the biological dam (Buddington et al., 2010; Baker et al., 2013; Paßlack et al., 2015), cross-fostering experiments show that environment, including diet, is the most important factor (Thompson et al., 2008; Bian et al., 2016). In our study, the faecal microbial diversity and composition of grower-finisher pigs later in life varied across litters, which may be due to similar early life colonization of littermates. Hence, if a common litter effect on faecal nutrient digestibility exists, postnatal common environment is likely more important than prenatal common environment. As common litter effects presented in literature are small (Ouweltjes et al., 2018) and we did not find evidence for a common litter effect on faecal digestibility of nutrients in our study, further studies are required to quantify and understand common litter effects on faecal digestibility of nutrients.

\subsubsection{Prediction accuracies}

Next to estimating the proportion of variance explained, this study also investigated the use of metagenomics to identify the accuracy of prediction of faecal nutrient digestibility values. We found that variation in faecal microbiota composition can be used to predict faecal digestibility of CP, DM, OM, CF and NSP and establish a rank among pigs that is useful for breeding purposes. The accuracy of prediction, however, is too low to reliably replace the golden standard in nutrition research for the determination of faecal nutrient digestibility via chemical analysis of faeces. In our case, the training data for the predictions in the leave-one-out cross-validation contained only 105 animals with one observed value each. Increasing the size of the training data will inevitably lead to an increase in prediction accuracy, albeit that the increase in accuracy will be subject to diminishing returns. In fact, the maximum prediction accuracy is expected to be equal to $\sqrt{m^{2}}$, similar to the maximum accuracy of predicting phenotypes based on estimated breeding values is expected to be $\sqrt{h^{2}}$ (Legarra et al., 2008). This suggest that increasing the training data could lead to maximum expected prediction accuracies as high as 0.97 for $\mathrm{CP}$, and 0.76 to 0.81 for CF, DM, OM and NSP. The prediction accuracy likely could also further be increased by using a dataset with more variation. The between-diet variation in 


\section{Faecal microbiota and nutrient digestion in pigs}

faecal nutrient digestibility values were larger than the within-diet variation, which is in line with the conclusion of Ouweltjes et al. (2018) that diet is the most important factor explaining phenotypic variance in faecal nutrient digestibility. Especially diets including different levels and sources of high fibre ingredients show large variation between diets in faecal digestibility of several nutrients beside fibre itself (Navarro et al., 2019). In addition, fibre inclusion level and composition have a pronounced effect on faecal microbiota composition (Castillo et al., 2007; Chen et al., 2014) but so does crude protein level (Zhou et al., 2016; Fan et al., 2017). Diet composition had a pronounced effect on faecal microbiota composition in our study as well. Thus, the prediction accuracy of the model is expected to increase if the dataset is expanded by including faecal nutrient digestibility values and faecal microbiota composition of pigs fed diets of different nutrient composition and ingredient sources.

\subsubsection{Implications}

We showed that faecal nutrient digestibility can be predicted based on variation in microbiota composition. Several studies investigated differences in faecal digestibility of nutrients between pig breeds and only found differences when the pigs were fed a low fibrous diet (Kemp et al., 1991; Urriola and Stein, 2012; Le Sciellour et al., 2018; Zhao et al., 2018). Within-breed genetic variation also appears to exist, as considerable sire effects on faecal digestibility of DM, OM, N and energy have been estimated (Noblet et al., 2013). The ultimate goal is to disentangle the interplay between host genetics, microbiota genetics and faecal nutrient digestibility, using genomic and metagenomic information simultaneously, which requires a larger dataset than we used in the present study. With the models presented in our study, however, individual pigs' faecal DM, OM, CP, CF and NSP digestibility can be predicted based on faecal microbiota composition. These predictions can then subsequently be used as phenotypes in genetic or genomic selection.

\subsection{Conclusion}

We obtained high microbiability values for faecal digestibility of $D M, O M, C P, C F$, and NSP, which shows that variation in faecal nutrient digestibility is strongly associated with variation in faecal microbiota composition. The accuracies of predicting individual faecal digestibility of nutrients based on faecal microbiota composition were too low if the interest is in substituting the golden standard for measuring individual nutrient digestibility. From the perspective of ranking animals in a genetic or genomic selection context, however, the accuracies of prediction are promising. 
In conclusion, metagenomics applied on faecal samples can possibly be used to predict faecal digestibility of DM, OM, CP, CF and NSP, and the predictions can subsequently be used as phenotypes in genetic or genomic selection.

\subsection{Acknowledgements}

This study was financially supported by the Dutch Ministry of Economic Affairs (TKI Agri \& Food project 12039; Public-private partnership "Breed\&Feed4Food" code BO-22.04-010-001). We also thank Schothorst Feed Research and the Breed4Food partner Topigs Norsvin for providing data.

\subsection{Conflict of interest}

The authors declare that there is no conflict of interest regarding the publication of this article.

\subsection{Data accessibility}

The data that support the findings of this study are available from the corresponding author upon reasonable request. 



\section{5}

\section{Between animal variation in dietary nitrogen and amino acid efficiency in grower-finisher pigs}

L.M.G. Verschuren ${ }^{1,2,3}$, M.P.L. Calus ${ }^{4}$, A.J.M. Jansman², J. van Milgen ${ }^{3,5}$

${ }^{1}$ Topigs Norsvin Research Center B.V., P.O. Box 43, 6640 AA Beuningen, The Netherlands; ${ }^{2}$ Wageningen University and Research, Wageningen Livestock Research, P.O. Box 338, $6700 \mathrm{AH}$ Wageningen, The Netherlands; ${ }^{3}$ Agrocampus Ouest, UMR1348 Pegase, Saint-Gilles, 35590, France; ${ }^{4}$ Wageningen University and Research, Animal Breeding and Genomics, P.O. Box 338, $6700 \mathrm{AH}$ Wageningen, The Netherlands; ${ }^{5}$ INRA, UMR1348 Pegase, Saint-Gilles, 35590, France 


\begin{abstract}
Overall feed efficiency of pork production is the result of optimizing diets on the one hand and of active selection of pigs for improved feed efficiency in breeding programs on the other hand. The aim of this study was to investigate the phenotypic variation in nitrogen and amino acid efficiency among grower-finishers pigs based on feed intake and growth curves, and estimate the heritability of nitrogen efficiency and its genetic and phenotypic correlation with feed efficiency traits. Phenotypic data were available for 2,137 pigs, 1081 intact boars, and 1056 gilts. Pigs were either offered a diet based on corn and soybean meal or a more fibrous diet based on wheat, barley, and by-products. Individual feed intake and bodyweight measurements were used to estimate empirical relationships in existing models and calculate nitrogen and amino acid efficiency of grower-finisher pigs during three dietary phases. There was a significant effect of diet and sex on the nitrogen and amino acid efficiencies, and nitrogen efficiencies were the highest for pigs fed the starter diet (average 55.3\%), and decreased subsequently for pigs fed the grower diet $(50.4 \%)$, and the finisher diet (42.1\%). After correction for diet and sex, there was still substantial variation in nitrogen and amino acid efficiency among the pigs. Heritability estimates of nitrogen efficiency ranged from 0.21 to 0.27 , and nitrogen efficiency had a small positive genetic correlation with ADG in pigs fed the starter diet (0.11), but decreased to a strong negative correlation in pigs fed the finisher diet $(-0.43)$. Nitrogen efficiency had a negative genetic correlation with FCR (-0.47 to $0.90)$ and ADFI (-0.17 to -0.92). In conclusion, variation in nitrogen and amino acid efficiencies can be calculated based on feed intake and growth patterns and selection for increased nitrogen efficiency is possible but would result in decreased FCR by reducing $A D F I$ and $A D G$.
\end{abstract}

Keywords: Nitrogen efficiency, Genetics, Swine, Growth curves, Feed intake 


\subsection{Introduction}

Overall feed efficiency of pork production is the result of optimizing diets on the one hand and of active selection of pigs for improved feed efficiency in breeding programs on the other hand. Worldwide, pigs are fed different types of diets based on available resources, yet even feed with the same ingredient composition differs in nutrient composition due to variation in nutrient composition between batches of the same ingredient. Feed efficiency traits, like feed conversion ratio (FCR), gain to feed, and residual feed intake (RFI) are mainly expressed as $\mathrm{kg}$ feed consumed versus kg bodyweight gained and do not account for differences in the nutrient composition of the diet. Body weight gain is a combination of the growth of muscles, adipose tissue, organs, and bones. Different body tissues require different nutrients for their growth, and the efficiency of nutrient utilisation possibly be improved by nutritional and breeding strategies. Muscle growth in pigs requires amino acids (AA) and energy, where excess AA are catabolized to yield energy and the excess nitrogen is excreted in urine (Pomar and Remus, 2019). When the interest is in having pigs that are efficient in muscle growth, measuring the $A A$ and nitrogen consumption and utilization of pigs would most likely be more beneficial than only considering the amount of feed consumed. In a nitrogen balance study, van der Peet-Schwering et al. (2021) showed that pigs divergently selected for their breeding value for protein deposition have different nitrogen efficiencies. To measure nitrogen efficiency, nitrogen consumption and excretion was estimated by measuring the nitrogen content of faeces and urine collected during 3 days (van der Peet-Schwering et al., 2021). Due to practicality and costs, nitrogen balance studies are not feasible for estimating nitrogen efficiency on a large scale. However, nitrogen efficiency can also be estimated with feed intake and bodyweight measurements to describe feed intake and growth curves using semi-mechanistic models, such as the INRAporc model (van Milgen et al., 2008).

The aim of the present study was to investigate the phenotypic variation in nitrogen and AA efficiency among grower-finishers pigs based on feed intake and growth curves, and estimate the heritability of nitrogen efficiency and its genetic and phenotypic correlation with feed efficiency traits. 


\subsection{Material and methods}

\subsubsection{Animals and experimental design}

Part of the data used in this study has been described previously by Godinho et al. (2018), Verschuren et al. (2018) and Verschuren et al. (2020). In brief, pigs originated from a three-way cross (i.e., Synthetic boar $x$ (Landrace $x$ Large White) sow) and phenotypic data were available for 2137 pigs (i.e., 1,081 intact boars and 1,056 gilts), coming from 289 litters. All pigs were kept under commercial conditions at the experimental facilities of Schothorst Feed Research B.V. (Lelystad, The Netherlands). Before the start of the study pigs were housed per litter, with approximately $17 \%$ of the pigs being cross-fostered. All pigs were offered the same diet during this period. The pigs entered the study at 54 to 69 days of age (day 0), in 29 groups over a time span of 117 weeks. Ten pigs were housed per pen and eight pens per compartment. One compartment was used per entrance date. Littermates were randomly distributed over the two diets and males and females were housed in separate pens, resulting in two pens per diet per sex per entrance date. The pigs were kept in the facilities until they reached a live weight at slaughter of approximately $120 \mathrm{~kg}$. Each pig was allowed a minimal space of $1 \mathrm{~m}^{2}$. Floors of the pens were $60 \%$ concrete and $40 \%$ slatted.

\subsubsection{Feeding strategy}

Two diets were used in the study, a diet based on corn and soybean meal (CS) as typically fed to commercial grower-finisher pigs in the Americas and a more fibrous diet based on wheat, barley, and by-products (WB) as typically fed in Europe (Table 5.1). Nutrient and AA composition of the diets were calculated with INRA tables based on the ingredient composition of the diet. The pigs were offered feed ad libitum throughout their life and received a starter diet from day 0 to day 25, a grower diet from day 26 to day 67, and a finisher diet from day 68 until they reached slaughter weight. A decrease of SID lysine to NE ratio in grower and finisher diets was achieved by exchanging soybean meal with corn for the CS diet, and peas with wheat for the WB diets. The experimental diets were produced by ABZ Diervoeding, Leusden, The Netherlands. 
Table 5.3 Ingredient and calculated composition of the diets fed to the grower-finisher pigs, as-fed basis

\begin{tabular}{|c|c|c|c|c|c|c|}
\hline \multirow[b]{2}{*}{ Item } & \multicolumn{2}{|c|}{$\begin{array}{c}\text { Starter } \\
\text { (day } 0 \text { to } 25 \text { ) }\end{array}$} & \multicolumn{2}{|c|}{$\begin{array}{c}\text { Grower } \\
\text { (day } 25 \text { to 67) }\end{array}$} & \multicolumn{2}{|c|}{$\begin{array}{c}\text { Finisher } \\
\text { (day } 67 \text { to end) }\end{array}$} \\
\hline & CS & WB & CS & WB & CS & WB \\
\hline \multicolumn{7}{|l|}{ Ingredient, $\mathrm{g} / \mathrm{kg}$} \\
\hline Corn & 647.1 & - & 698.4 & - & 755.1 & - \\
\hline Corn gluten feed & 18.1 & - & 25 & 50 & 50 & 50 \\
\hline Soybean meal (48\% CP) & 240.5 & 100 & 180.5 & 21.5 & 98.3 & - \\
\hline Soybean hulls & - & - & - & 14.3 & - & 50 \\
\hline Soybean oil & - & 25 & - & 0.3 & - & - \\
\hline Barley & - & 200 & - & 100 & - & 150 \\
\hline Wheat & - & 321.9 & - & 400 & - & 350 \\
\hline Wheat middlings & - & - & - & 50 & - & 125 \\
\hline Rapeseed meal & - & 63 & - & 80 & - & 100 \\
\hline Sunflower seed meal & - & 80 & - & 80 & - & 21.9 \\
\hline Palm kernel meal & - & - & - & 50 & - & 50 \\
\hline Palm oil & 5 & 17.3 & 5 & 16 & 5 & 5 \\
\hline Field peas & - & 120 & - & 29.4 & - & - \\
\hline Sugarcane molasses & 40 & 30 & 50 & 50 & 50 & 50 \\
\hline Poultry fat & - & - & - & 27.5 & - & 29.4 \\
\hline Monocalcium phosphate & 6.7 & 5.3 & 2 & - & 0.7 & - \\
\hline Salt & 2.7 & 2.1 & 2.4 & 1.8 & 1.8 & 2.1 \\
\hline Sodium bicarbonate & - & 1.1 & 1 & 1 & 3.4 & - \\
\hline Phytase $^{1}$ & 5 & 5 & 5 & 5 & 5 & 1.9 \\
\hline Limestone & 11.6 & 10.9 & 9.4 & 8.9 & 9.9 & 4 \\
\hline AA premix ${ }^{2}$ & 17.3 & 12.5 & 17.3 & 10.2 & 16.7 & 6.7 \\
\hline Lys + Trp premix & 7.8 & 4.3 & 8.3 & 3.6 & 9.2 & - \\
\hline Lys HCl (L 79\%) & 2.4 & 3.8 & 2.2 & 4.3 & 1.9 & 3.3 \\
\hline Met (DL 99\%) & 1.6 & 1.3 & 1.4 & 0.7 & 0.8 & 0.1 \\
\hline Thr (L 98\%) & 1.5 & 1.7 & 1.5 & 1.6 & 1.5 & 0.9 \\
\hline Val (L 10\%) & - & 1.4 & - & - & - & - \\
\hline Vitamin premix3 & 1 & 1 & - & - & - & - \\
\hline Vitamin-trace mineral premix4 & 5 & 5 & 4 & 4 & 4 & 4 \\
\hline \multicolumn{7}{|l|}{ Nutrient composition, g/kg5 } \\
\hline $\mathrm{NE}, \mathrm{MJ} / \mathrm{kg}$ & 9.99 & 9.74 & 10.22 & 9.59 & 10.47 & 9.26 \\
\hline Dry matter & 870.1 & 879.1 & 867.1 & 879.4 & 866.8 & 877.0 \\
\hline Ash & 53.3 & 56.5 & 43.4 & 47.5 & 40.3 & 42.4 \\
\hline Crude protein & 193.1 & 189.0 & 174.7 & 184.7 & 157.1 & 165.6 \\
\hline Crude fat & 34.4 & 57.0 & 35.4 & 63.7 & 36.6 & 56.7 \\
\hline Starch & 417.9 & 352.6 & 452.0 & 329.8 & 492.7 & 333.3 \\
\hline Sugar & 49.2 & 48.4 & 49.8 & 53.4 & 43.9 & 53.7 \\
\hline NSP & 122.2 & 175.6 & 111.7 & 200.3 & 96.2 & 225.3 \\
\hline $\mathrm{Ca}$ & 8.6 & 8.6 & 6.2 & 6.5 & 5.9 & 4.8 \\
\hline$P$ & 4.8 & 5.6 & 3.6 & 4.7 & 3.0 & 4.7 \\
\hline SID Lys & 12.6 & 11.7 & 11.5 & 9.8 & 9.8 & 7.4 \\
\hline
\end{tabular}




\section{Variation in nitrogen and amino acid efficiency in pigs}

\begin{tabular}{lcccccc} 
Amino acid composition, \% of SID Lys & & & & & \\
Met & 43.2 & 32.3 & 43.1 & 36.5 & 40.0 & 38.9 \\
Cys & 21.6 & 23.7 & 22.1 & 29.9 & 24.6 & 37.8 \\
Trp & 45.2 & 33.4 & 46.2 & 33.5 & 55.8 & 18.2 \\
Thr & 74.7 & 58.0 & 76.1 & 65.1 & 82.6 & 89.6 \\
Phe & 63.3 & 59.5 & 62.3 & 72.2 & 66.8 & 85.4 \\
Tyr & 46.4 & 38.2 & 46.6 & 49.0 & 52.1 & 59.5 \\
Leu & 118.5 & 86.8 & 124.0 & 126.7 & 149.6 & 154.8 \\
Ile & 52.7 & 51.0 & 50.5 & 57.2 & 50.8 & 66.1 \\
Val & 58.8 & 70.5 & 57.5 & 68.3 & 60.0 & 81.5 \\
His & 33.4 & 31.4 & 32.5 & 34.7 & 33.3 & 41.4 \\
Arg & 80.6 & 89.8 & 74.4 & 91.5 & 67.7 & 100.4 \\
\hline
\end{tabular}

$\mathrm{CS}=$ a diet based on corn/soybean meal; WB = a diet based on wheat/barley/by-products; AA = amino acid; Lys = Lysine; Met = Methionine; Cys = Cystine; Trp = Tryptophan; Thr = Threonine; Phe = Phenylalanine; Tyr = Tyrosine, Leu = Leucine, Ile = Isoleucine, Val = Valine; His = Histidine; $\mathrm{Arg}=$ Arginine; NE = Net energy, NSP = Non-starch polysaccharides; SID = Standardized ileal digestible; $\mathrm{Cys}=$ Cystine.

${ }^{1}$ Phyzyme XP (Dupont, Wilmington, Delaware, United states of America)/ Assumed P released 500 FTU: $1.12 \mathrm{~g}$ digestible $\mathrm{P} / \mathrm{kg}$.

${ }^{2}$ Mixture of free Lys, Met, Thr, Trp, and Val to equalize dietary levels of SID amino acids relative to the net energy value of the diet. ${ }^{4}$ Supplied per kilogram of feed: $2500 \mathrm{IU}$ of vitamin A, 500 IU of vitamin D3, and 5 IU of vitamin E (Mervit AD3E; PreMervo, Utrecht, the Netherlands).

${ }^{3}$ Supplied per kilogram of feed: $12 \mathrm{mg}$ of Fe (ferrous sulphate), $10 \mathrm{mg}$ of $\mathrm{Mn}$ (manganous oxide), $0.04 \mathrm{mg}$ of Co cobalt oxide), $0.12 \mathrm{~g}$ of $\mathrm{Ca}, 0.0501 \mathrm{~g}$ of $\mathrm{P}, 0.04 \mathrm{mg}$ of I (potassium iodide), $1000 \mathrm{IU}$ of vitamin A, $100 \mathrm{IU}$ of vitamin D3, $5 \mathrm{IU}$ of vitamin E, $0.4 \mathrm{mg}$ of vitamin B1, $0.8 \mathrm{mg}$ of vitamin B2, $2 \mathrm{mg}$ of pantothenic acid, $4 \mathrm{mg}$ of niacin, $0.4 \mathrm{mg}$ of vitamin B6, $0.2 \mathrm{mg}$ of folate, $0.003 \mathrm{mg}$ of vitamin B12, $10 \mathrm{mg}$ of vitamin C, $0.01 \mathrm{mg}$ of biotin, $0.2 \mathrm{mg}$ of vitamin $\mathrm{K} 3$, and 40 $\mathrm{mg}$ of choline (Mervit Sporavit; PreMervo, Utrecht, the Netherlands).

${ }^{4}$ Supplied per kilogram of premix: $0.4 \mathrm{~g}$ of $\mathrm{Ca}, 15 \mathrm{mg}$ of $\mathrm{Cu}$ (copper sulphate), $80 \mathrm{mg}$ of $\mathrm{Fe}$ (ferrous sulphate), $24 \mathrm{mg}$ of $\mathrm{Mn}$ (manganous oxide), $62 \mathrm{mg}$ of $\mathrm{Zn}$ (zinc oxide), $0.04 \mathrm{mg}$ of Co (cobalt oxide), $0.4 \mathrm{mg}$ of I (potassium iodide), $0.2 \mathrm{mg}$ of Se (sodium selenite), $7500 \mathrm{IU}$ of vitamin A, $1500 \mathrm{IU}$ of vitamin D3, $25 \mathrm{IU}$ of vitamin E, $4 \mathrm{mg}$ of vitamin B2, $6 \mathrm{mg}$ of pantothenate, $30 \mathrm{mg}$ of niacin, $0.02 \mathrm{mg}$ of vitamin B12, and $0.752 \mathrm{mg}$ of vitamin K3 (Mervit START M220; PreMervo, Utrecht, the Netherlands).

${ }^{5}$ Based on chemical composition, digestibility, and energy values for pigs in INRAporc. 


\subsubsection{Measurements and sampling}

The experimental facilities (Schothorst Feed Research B.V.) were equipped with IVOG feeding stations (INSENTEC, Marknesse, The Netherlands) that register individual feed intake of group-housed pigs. All pigs had ear tags with unique numbering and individual feed intake records were available for all pigs for each day on test. Pigs were weighted at day 0, day 56, and at the end of the study (day $104 \pm$ 6.7). A subset of animals ( $n=200$ ) was weighed at change of diets (day 25 and day 67). The average daily feed intake (ADFI) was calculated as the cumulated individual daily feed intake records divided by the timespan over which the feed intake records were collected. The average daily gain (ADG) was calculated as the difference between BW measurements divided by the timespan between the measurements, whereas the feed conversion ratio (FCR) was calculated as the ratio between ADFI and ADG.

\subsubsection{Amino acid efficiency calculation}

Daily feed intake and bodyweight measured during the experiment were used to determine body protein deposition and feed intake curves using the INRAporc model (van Milgen et al., 2008) in three steps. (1) Missing feed intake observations were estimated by calculating the average of the two feed intake observations before and after the missing observations. Missing feed intake data at the beginning and end of the period were ignored. The ad libitum net energy intake curve was described as a Gamma function of maintenance requirement of energy so that an adult nonproducing pig would eat for maintenance requirement of energy:

Net energy intake $(M J / d)=(a \times(b \times B W(k g) \times \exp (-b \times$ $B W(k g)))+1) \times c \times(B W(k g))^{60}$

where $B W$ is the bodyweight and $c$ is a constant describing the net energy requirement for maintenance equal to $0.75 \mathrm{MJ} / \mathrm{BW}^{0.60} / \mathrm{d}$ (van Milgen et al., 2008). The $a$ an $b$ parameters were estimated for every individual pig based on measured daily feed intake and net energy content of the diet. Body protein deposition was estimated using a Gompertz function based on the actual protein mass (and not as an explicit function of time):

Protein depositon $\left(\frac{g}{d}\right)=$ precocity $\cdot P(g) \cdot \ln \left(\frac{P_{\text {maturity }}(g)}{P(g)}\right)$, where precocity is the shape parameter of the Gompertz function, $P$ is the actual body protein mass, and $P_{\text {maturity }}$ the protein mass at maturity (van Milgen et al., 2008). The Gompertz function was parameterized to include the protein mass at the start of the test $(P O)$, precocity, and the mean protein deposition during the test 


\section{Variation in nitrogen and amino acid efficiency in pigs}

(MeanPD). PO, precocity, and MeanPD were estimated for every individual pig based on measured body weight gain.

(2) Model parameters were obtained by comparing the observed feed intake and body weight data with model predictions using the statistical algorithm of the INRAporc software, and it was assumed that the AA supply was not limiting protein deposition. The estimates of the parameters for feed intake (i.e., $a$ and $b$ ) and body protein deposition (i.e., precocity, $P O$, and meanPD) were used to simulate feed intake, body protein, and body lipid composition over a 100 days period. Data of some animals were deleted $(n=185)$ because of low correlation between calculated and measured feed intake $\left(R^{2}<0.9\right)$ or bodyweight $\left(R^{2}<0.9975\right)$ in regression, or because of extreme parameters for the curves $(0>a>10,0>b>0.03)$. These low correlations or biologically non-realistic parameter values may be the results of pigs showing some irregular feed intake or growth pattern (e.g., due to perturbations), whereas the models assume a non-disturbed growth period.

(3) Because diets were formulated to meet or exceed $A A$ requirements, the $A A$ and nitrogen efficiency estimates are apparent values. The standardized ileal digestible (SID) AA intake was calculated as:

AA fed $(g / d)=$ Feed intake $(g / d) \times A A$ concentration diet $(S I D g / k g$ as is),

the $A A$ required for protein deposition was based on the (constant) AA composition of body protein of a pig (Le Bellego and Noblet, 2002), and was calculated as:

$A A$ deposited $(g / d)=A A$ composition of body protein $\times$ protein deposition $(g / d)$,

the apparent AA efficiency was calculated based on the ratio between the deposited $A A$ and the $A A$ intake:

$A A$ efficiency $=A A$ deposited $(g / d) /(A A$ fed $(S I D g / d)$,

and the apparent nitrogen efficiency was calculated similarly as:

Nitrogen efficiency $=$ protein deposition $(g / d) /($ Feed intake $(g / d) \times$ crude protein concentration diet $(\mathrm{g} / \mathrm{kg}$ as $-i s)) \times 100 \%$.

The $A A$ and $\mathrm{N}$ efficiencies change from day to day, but are typically the highest at the start of a production phase, and these values were further used in the analyses. 


\subsubsection{Statistical analysis}

The data resulted from an experimental set-up as a split-plot design in a $2 \times 2$ factorial arrangement, the two factors being diet (CS vs. WB diets) and sex (intact boars vs. gilts). Individual pigs were considered the experimental unit. We investigated the difference in $A A$ and nitrogen efficiency between the two diets and sexes, and obtained their phenotypic variation after correction for these factors. The statistical model was:

$y_{i j k \text { lmno }}=\mu+\beta_{j}$ age $_{i}+\beta_{k} B W_{i}+\operatorname{sex}_{l}+\operatorname{diet}_{m}+\operatorname{sex}_{l} \cdot \operatorname{diet}_{m}+\operatorname{group}_{n}+$ group $_{n} \cdot$ diet $_{m}+$ pen $_{o}+e_{i j k l m o}$

where:

$x_{i j k l m o}=$ phenotype of animal $\mathrm{i}(\mathrm{i}=1 \ldots$ 1952)

$\mu \quad=$ overall mean

$\beta_{j} \quad$ = regression coefficient representing age in days

$a e_{i} \quad=$ age at start of experiment of individual pig $\mathrm{i}$

$\beta_{k} \quad=$ regression coefficient representing body weight at start of experiment

$B W_{i} \quad=$ body weight at start of experiment of individual pig $\mathrm{i}$

$\operatorname{sex}_{l}=$ code for sex $(l=$ boar or gilt $)$

$\operatorname{diet}_{m}=$ code for diet $(\mathrm{m}=\mathrm{CS}$ or WB)

$\operatorname{group}_{n}=$ code for contemporary group $(n=1 \ldots 29)$

pen $_{o} \quad=$ common effect of pigs from the same pen in the experiment $(p=1 \ldots 233)$

$e_{i j k l m}=$ error

$\mu$, age, body weight, sex, diet, group were modelled as fixed effects, pen and $e$ were modelled as random effects.

Univariate analyses were performed to estimate the variance components and heritability values of FCR, ADG, ADFI, and nitrogen efficiency. Phenotypic correlations $\left(r_{p}\right)$ and genetic correlations $\left(r_{g}\right)$ were estimated with bivariate analyses. A linear mixed model, implemented in ASReml (Gilmour et al., 2015), was used for the univariate and bivariate analyses, as follows:

$y_{i j k l m n o}=\mu+\beta_{j}$ age $_{i}+\beta_{k} B W_{i}+\operatorname{sex}_{l}+\operatorname{diet}_{m}+\operatorname{sex}_{l} \cdot \operatorname{diet}_{m}+\operatorname{group}_{n}+$ group $_{n} \cdot$ diet $_{m}+$ Pen $_{o}+$ litter $_{p}+$ animal $_{i}+e_{i j k l m n o p}$

where $y, \mu, \beta_{\mathrm{j}}$, age $\mathrm{e}_{\mathrm{i}}, \mathrm{BW}_{\mathrm{i}}$, sex , diet $_{\mathrm{m}}$, group $\mathrm{g}_{\mathrm{n}}$, pen $_{\circ}$ and $\mathrm{e}_{\mathrm{ijklmno}}$ are as described previously. litter $r_{o}$ is the common effect of pigs from the same litter ( $0=1 \ldots 407$ ), and animal $_{i}$ is the effect of individual pig i. $\mu$, age, body weight, sex, diet, group were modelled as fixed effects, litter, pen, animal and $e$ were modelled as random effects, assuming distributions of $N\left(0, \mathbf{l}_{\text {litter }} \sigma^{2}\right.$ litter $), N\left(0, \mathbf{I}_{\text {pen }} \sigma_{\text {pen }}^{2}\right), N\left(0, \mathbf{A} \mid \sigma_{\text {animal }}{ }^{2}\right)$, and $N\left(0, \mathbf{I}_{\text {records }} \sigma^{2}{ }_{\text {e }}\right)$, respectively, where $\sigma^{2}$ litter was the common litter variance, $\sigma^{2}$ pen was the common pen variance, $\sigma^{2}$ animal was the variance due to individual animal effects, 
$\sigma^{2}$ e was the error variance, $\mathbf{A}$ is the additive genetic relationship matrix, and I denotes the identity matrix, whose dimensions were equal to the number of sexes, diets, litters, pens, animals, and records, respectively.

\subsection{Results}

\subsubsection{Variation in amino acid and nitrogen efficiencies}

The nitrogen and AA efficiencies the first day after a dietary switch differed between the CS and WB diet, and between the sexes (Table 5.2). However, there was an interaction between diet and sex. The apparent efficiency of nitrogen and of all AA decreased from starter to grower to finisher diet, where tryptophan had the lowest efficiency in almost all diets. Histidine had the highest efficiency for both diets during the starter and grower phases. During the finisher phase, arginine had the highest efficiency in the CS diet, whereas lysine had the highest efficiency in the WB diet. Lysine efficiency ranged from 43 to $65 \%$ and was higher for the animals fed the WB diets compared to the animals fed the CS diet. The statistical models explained 27 to $84 \%$ of the variation in nitrogen and $A A$ efficiencies, and although there were substantial effects of diet and sex, there was still considerable between-animalvariation as shown in Figure 5.1. Phenotypic variation in nitrogen and AA efficiency after correction for fixed effects was highest for the starter diets and lowest for the finisher diets.

Table 5.2 Nitrogen and amino acid efficiency per feeding phase of male and female growerfinisher pigs fed one out of two experimental diets, and the explained variance by the models ${ }^{1}$

\begin{tabular}{|c|c|c|c|c|c|c|c|c|c|}
\hline \multirow{2}{*}{ Amino acid } & \multicolumn{2}{|c|}{ CS } & \multicolumn{2}{|c|}{ WB } & \multicolumn{3}{|c|}{ P-value } & \multicolumn{2}{|c|}{ Variance } \\
\hline & $B$ & G & B & $\mathrm{G}$ & Diet & Sex & $D * S$ & RMSE & Exp \\
\hline \multicolumn{10}{|l|}{ Starter } \\
\hline Nitrogen & 56.3 & 53.9 & 57.6 & 53.4 & ns & $* * *$ & $*$ & 6.6 & 26.7 \\
\hline Lys & 59.6 & 57.1 & 65.0 & 60.2 & $*$ & $* * *$ & $* *$ & 7.3 & 30.3 \\
\hline Met & 37.3 & 35.7 & 54.4 & 50.4 & $* * *$ & $* * *$ & $* * *$ & 5.5 & 69.7 \\
\hline Met+Cys & 38.4 & 36.8 & 48.6 & 45.0 & $* * *$ & $* * *$ & $* * *$ & 5.1 & 52.0 \\
\hline Tryp & 18.0 & 17.2 & 26.6 & 24.6 & $* * *$ & $* * *$ & $* * *$ & 2.7 & 70.8 \\
\hline Thr & 42.4 & 40.6 & 59.6 & 55.2 & $* * *$ & $* * *$ & $* * *$ & 6.1 & 65.9 \\
\hline Phe & 51.1 & 49.0 & 59.3 & 55.0 & $* * *$ & $* * *$ & $* *$ & 6.5 & 38.5 \\
\hline Phe+Tyr & 51.9 & 49.6 & 63.5 & 58.8 & $* * *$ & $* * *$ & $* *$ & 6.8 & 47.1 \\
\hline Leu & 51.8 & 49.6 & 77.2 & 71.5 & $* * *$ & $* * *$ & $* * *$ & 7.7 & 71.5 \\
\hline Ile & 56.3 & 53.8 & 63.4 & 58.7 & $* * *$ & $* * *$ & $* *$ & 7.0 & 34.3 \\
\hline Val & 68.1 & 65.1 & 61.8 & 57.3 & $*$ & $* * *$ & ns & 7.6 & 36.5 \\
\hline His & 71.5 & 68.4 & 82.9 & 76.8 & $* * *$ & $* * *$ & $* *$ & 9.1 & 38.6 \\
\hline Arg & 66.6 & 63.7 & 65.1 & 60.4 & ns & $* * *$ & $*$ & 7.7 & 28.0 \\
\hline
\end{tabular}




\begin{tabular}{|c|c|c|c|c|c|c|c|c|c|}
\hline \multicolumn{10}{|l|}{ Grower } \\
\hline Nitrogen & 51.1 & 48.1 & 53.4 & 49.2 & ns & $* * *$ & $*$ & 4.8 & 32.3 \\
\hline Lys & 53.9 & 50.7 & 62.3 & 57.5 & $* * *$ & $* * *$ & $* *$ & 5.3 & 47.6 \\
\hline Met & 33.7 & 31.8 & 46.1 & 42.6 & $* * *$ & $* * *$ & $* * *$ & 3.7 & 73.1 \\
\hline Met+Cys & 34.5 & 32.5 & 39.2 & 36.2 & $* * *$ & $* * *$ & * & 3.4 & 44.3 \\
\hline Tryp & 15.9 & 15.0 & 25.4 & 23.4 & $* * *$ & $* * *$ & $* * *$ & 1.9 & 84.4 \\
\hline Thr & 37.6 & 35.4 & 50.9 & 47.0 & $* * *$ & $* * *$ & $* * *$ & 4.1 & 71.9 \\
\hline Phe & 47.0 & 44.2 & 46.8 & 43.3 & ns & $* * *$ & ns & 4.3 & 31.1 \\
\hline Phe+Tyr & 47.2 & 44.4 & 49.0 & 45.3 & ns & $* * *$ & ns & 4.4 & 31.7 \\
\hline Leu & 44.7 & 42.1 & 50.6 & 46.8 & $* * *$ & $* * *$ & $*$ & 4.4 & 43.5 \\
\hline Ile & 53.1 & 49.9 & 54.2 & 50.0 & $\mathrm{~ns}$ & $* * *$ & ns & 4.9 & 30.9 \\
\hline Val & 62.9 & 59.2 & 61.2 & 56.5 & ns & $* * *$ & ns & 5.7 & 32.7 \\
\hline His & 66.4 & 62.5 & 71.9 & 66.4 & $*$ & $* * *$ & $*$ & 6.3 & 36.1 \\
\hline Arg & 65.1 & 61.3 & 61.3 & 56.6 & $*$ & $* * *$ & ns & 5.8 & 37.0 \\
\hline \multicolumn{10}{|l|}{ Finisher } \\
\hline Nitrogen & 41.5 & 38.5 & 45.5 & 42.6 & ns & $* * *$ & ns & 4.1 & 41.9 \\
\hline Lys & 45.9 & 42.6 & 60.0 & 56.3 & $* * *$ & $* * *$ & ns & 5.0 & 69.1 \\
\hline Met & 31.0 & 28.8 & 41.7 & 39.1 & $* * *$ & $* * *$ & ns & 3.4 & 72.4 \\
\hline Met+Cys & 29.7 & 27.6 & 32.7 & 30.7 & ns & $* * *$ & ns & 2.9 & 43.0 \\
\hline Tryp & 11.2 & 10.4 & 45.0 & 42.1 & $* * *$ & $* *$ & $* * *$ & 3.1 & 96.3 \\
\hline Thr & 29.6 & 27.4 & 35.6 & 33.4 & $* * *$ & $* * *$ & ns & 3.1 & 57.7 \\
\hline Phe & 37.3 & 34.7 & 38.2 & 35.8 & ns & $* * *$ & ns & 3.5 & 34.8 \\
\hline Phe+Tyr & 36.8 & 34.2 & 39.5 & 37.0 & ns & $* * *$ & ns & 3.6 & 39.0 \\
\hline Leu & 31.6 & 29.4 & 39.9 & 37.4 & $* * *$ & $* * *$ & ns & 3.4 & 64.7 \\
\hline Ile & 44.9 & 41.7 & 45.1 & 42.3 & ns & $* * *$ & ns & 4.2 & 34.3 \\
\hline Val & 51.4 & 47.7 & 49.4 & 46.3 & $* *$ & $* * *$ & ns & 4.7 & 36.0 \\
\hline $\mathrm{His}$ & 55.3 & 51.3 & 58.1 & 54.4 & ns & $* * *$ & ns & 5.3 & 36.7 \\
\hline Arg & 61.0 & 56.6 & 53.8 & 50.4 & $* * *$ & $* * *$ & ns & 5.4 & 48.1 \\
\hline \multicolumn{10}{|c|}{$\begin{array}{l}\text { CS = a diet based on corn/soybean meal; WB = a diet based on wheat/barley/by-products; B = } \\
\text { boars; G = gilts; D*S = interaction between diet and sex effects; } \operatorname{Exp}=\text { explained variance (\%) } \\
\text { by the statistical model; Lys = Lysine; Met = Methionine; Cys = Cystine; } \operatorname{Trp}=\text { Tryptophan; Thr } \\
\text { = Threonine; Phe = Phenylalanine; Tyr = Tyrosine, Leu = Leucine, lle = Isoleucine, Val = Valine; } \\
\text { His = Histidine; Arg = Arginine; } \\
{ }^{1} \text { Statistical model including the effect of diet, sex, the interaction between diet and sex, group, } \\
\text { the interaction between diet and group, body weight and age at start of the experiment, and } \\
\text { pen; }\end{array}$} \\
\hline
\end{tabular}




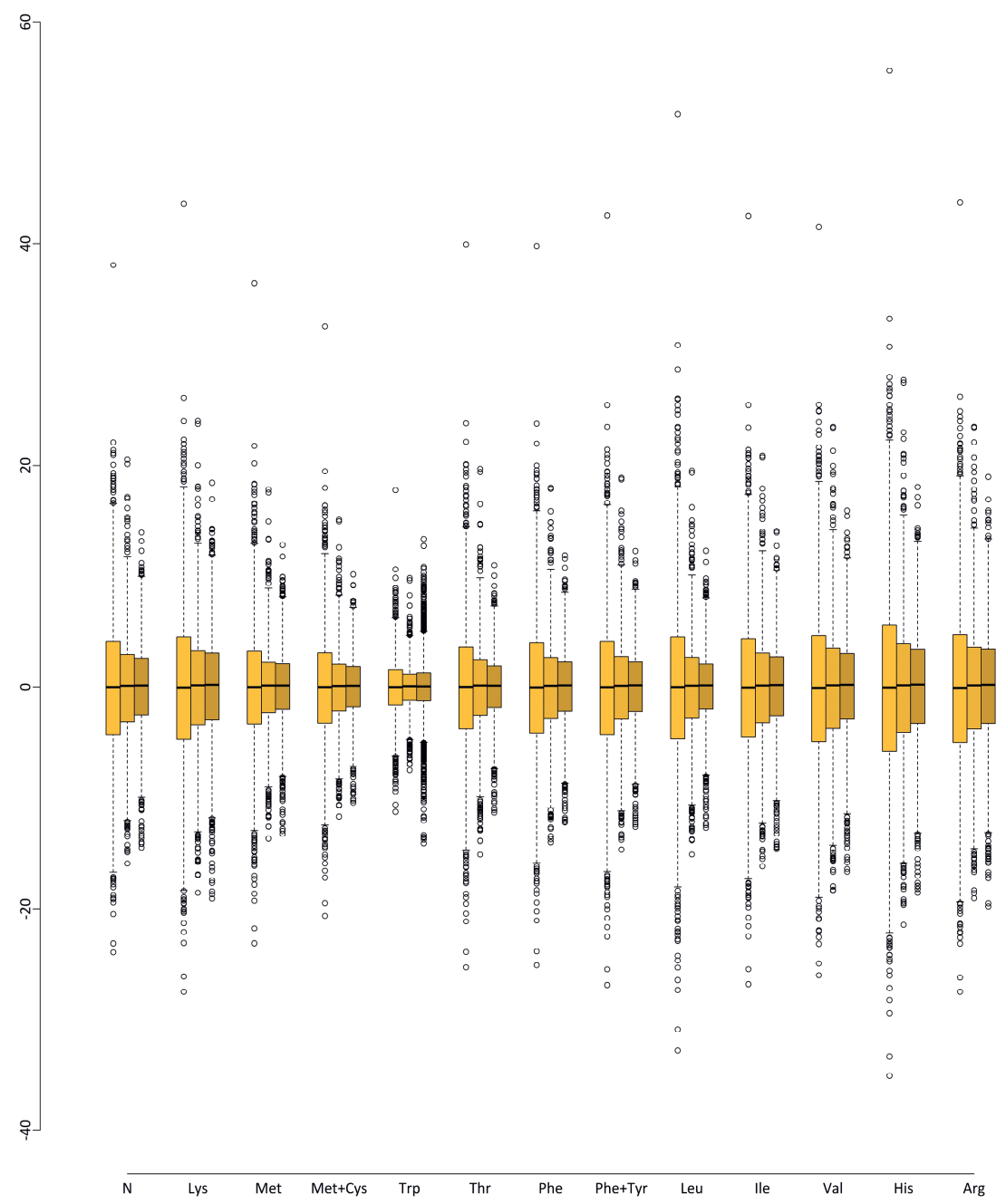

Figure 5.1 Variation in corrected ${ }^{1}$ nitrogen and amino acid efficiency(\%) at the first day of a feeding phase.

${ }^{1}$ Phenotypic variation in nitrogen and amino acid efficiency after statistical correction for diet, sex, the interaction between diet and sex, group, the interaction between diet and group, body weight and age at start of the experiment, and pen. $\mathrm{N}=$ Nitrogen; Lys = Lysine; Met = Methionine; Cys = Cystine; $\operatorname{Trp}=$ Tryptophan; Thr = Threonine; Phe = Phenylalanine; $\mathrm{Tyr}=$ Tyrosine, Leu = Leucine, Ile = Isoleucine, Val = Valine; His = Histidine; Arg = Arginine . 


\subsubsection{Genetic parameters}

Estimates of the heritability, and phenotypic and genetic correlations are presented in Table 5.3. The heritability of nitrogen efficiency per dietary phase ranged from 0.21 to 0.27 . Nitrogen efficiency had a positive genetic correlation of 0.11 with ADG in the starter phase, but the correlation decreased to -0.11 in the grower phase, and to -0.43 in the finisher phase. A similar pattern occurred for the genetic correlation between nitrogen efficiency and ADFI and FCR, which started at -0.17 and -0.47 in the starter phase and decreased to -0.92 and -0.90 in the finisher phase, respectively. The genetic correlation of nitrogen efficiency between the dietary phases was high between the starter and grower diets, but low between the finisher and the starter and grower diet. Phenotypic correlations generally showed the same patterns as the genetic correlations, albeit that their values were generally closer to 0 . Overall, the strongest genetic correlation of nitrogen efficiency with ADG, ADFI, and FCR was observed in the finisher phase.

Table 5.3 Heritability (diagonal), phenotypic (above diagonal) and genetic (below diagonal) correlations between traits ${ }^{1}$ measured over the total grower-finisher period and the nitrogen efficiency by feeding phase

\begin{tabular}{lccccccc}
\hline Trait $^{1}$ & \multicolumn{3}{c}{ Overall period } & & \multicolumn{3}{c}{ Nitrogen efficiency } \\
\cline { 2 - 3 } \cline { 7 - 8 } & ADG & FCR & ADFI & & Starter & Grower & Finisher \\
\hline Overall period & & & & & & & \\
ADG (g/d) & $\mathbf{0 . 2 7}$ & -0.36 & 0.76 & & 0.10 & 0.08 & 0.13 \\
FCR (kg/kg) & -0.03 & $\mathbf{0 . 3 0}$ & 0.32 & & -0.25 & -0.54 & -0.85 \\
$\quad$ ADFI (kg/d) & 0.82 & 0.55 & $\mathbf{0 . 4 3}$ & & -0.08 & -0.30 & -0.46 \\
Nitrogen efficiency (\%) & & & & & & \\
$\quad$ Starter & 0.11 & -0.47 & -0.17 & & $\mathbf{0 . 2 2}$ & 0.90 & -0.06 \\
$\quad$ Grower & -0.11 & -0.68 & -0.50 & & 0.92 & $\mathbf{0 . 2 7}$ & 0.33 \\
Finisher & -0.43 & -0.90 & -0.92 & & 0.13 & 0.47 & $\mathbf{0 . 2 1}$
\end{tabular}

${ }^{1} \mathrm{ADG}=$ average daily gain; $\mathrm{ADFI}$ = average daily feed intake; $\mathrm{FCR}$ = feed conversion rate. 


\section{Variation in nitrogen and amino acid efficiency in pigs}

\subsection{Discussion}

This study investigated the phenotypic variation in apparent nitrogen and AA efficiency among grower-finishers pigs based on feed intake and protein deposition patterns estimated with empirical models. To do so, we collected individual daily feed intake data and weighted pigs at the start, middle, and end of the growerfinisher phase. Some pigs were weighed twice additionally. Three datapoints is the bare minimum to estimate a non-linear relationship, as with two datapoints only a linear relationship can be described. However, a larger number of available datapoints in time would result in an estimate that more closely follows the real dynamics of protein deposition of a pig, as the bodyweight measured at the middle of the experiment now fully determines the shape of the growth curve. The limited number of observations also contributed to obtaining a number of animals for which the model parameters appeared biologically not very plausible. Because bodyweight is the accumulation of gain, the feed intake was also expressed as cumulative feed intake as suggested by van Milgen et al. (2008). Hence, daily individual feed intake measurements are needed to use our approach of estimating the feed intake curve.

\subsubsection{Variation in amino acid and nitrogen efficiency}

We found clear differences in AA efficiency between the diets and sexes, with higher efficiencies for the pigs fed the WB diet compared to the CS diet. Also, all AA efficiencies were higher for boars than for gilts. The INRAporc model assumes a fixed maximum achievable efficiency for each AA so that the AA supply can affect protein deposition and thus growth. In the present study, these maximum efficiencies were not used to affect protein deposition estimations in the INRAporc model. The average efficiencies of leucine and isoleucine of boars fed the WB diet were above the maximum efficiency of 70 and $60 \%$, respectively. However, as indicated, the efficiency of $A A$ utilisation was assessed at the first day of a production phase when the efficiency of pigs is highest. For all other $A A$, the average efficiencies were below the maximum values as estimated by van Milgen et al. (2008). Still, we found phenotypic variation in AA efficiency after correcting for the effect of diet and sex, resulting in AA efficiencies of several animals above the maximum values estimated by van Milgen et al. (2008). The diets fed to the pigs in our study had a fixed dietary ingredient composition during the more than two years duration of the experiment. One batch of feed was analysed by wet chemistry for crude protein (Sevillano et al., 2018) and the crude protein levels measured were below the calculated level according to dietary ingredient composition. In addition, the AA to lysine ratio of several AA were below the recommended levels in literature (Van der Peet- 
Schwering and Bikker, 2018). Also the lysine to net energy ratio was below the recommendations of $0.85 \mathrm{~g}$ SID lysine/MJ NE for the boars fed a grower WB diet (Van der Peet-Schwering and Bikker, 2018). Hence, it is possible that the protein deposition of several pigs in our experiment was limited by the dietary AA and protein supply of at least one diet fed during the grower-finisher period. Nitrogen retention efficiency typically increases when an AA, such as lysine or threonine, is actually limiting protein deposition (Fabian et al., 2002; Remus et al., 2020), but is constant at decreasing suboptimal levels of lysine, threonine, methionine + cystine, and tryptophan (Heger et al., 2002). A higher AA efficiency when pigs are fed AA on a suboptimal level could most likely partly explain the AA high efficiency values we found in our study.

The AA efficiencies were calculated based on the protein deposition and feed intake curves assuming a constant $A A$ composition of body protein. However, the $A A$ composition of whole body protein can change because there are different proteins in the body (e.g., myosin, actin, collagen), with different AA compositions. CondeAguilera et al. (2010) showed that a deficient supply of methionine affects tissues and their AA composition differently. Also threonine supply influences $A A$ composition and total protein content of body tissue differently between tissues (Remus et al., 2020). As the AA supply influences the AA composition of body protein, the assumption of a constant $A A$ composition is likely invalid, but the magnitude of the error is unknown. Hence, by calculating AA efficiency based on protein deposition and feed intake curves we possibly over- or underestimated the specific AA efficiency, but the calculated nitrogen efficiency is likely representative for the actual nitrogen efficiency.

\subsubsection{Genetic parameters}

In estimating the AA efficiency, the dietary AA supply was assumed to be non-limiting for protein deposition. Pigs were offered feed ad libitum and protein deposition was assumed to be determined by the phenotypic potential for protein deposition. Because the pigs were fed the same diet, differences in AA and nitrogen efficiencies reflect differences in the phenotypic potential for protein deposition. Pigs with a lower-than-average potential received a diet with excess of protein and $A A$, resulting in lower-than-average apparent efficiencies. The word "apparent" is important here, because it is the supply of protein and AA in the diet that determined the efficiencies. If a diet was fed to these pigs which was actually limiting in one or more AA for all pigs, the animals would have been "forced" to express their maximum efficiency of AA utilisation. 


\section{Variation in nitrogen and amino acid efficiency in pigs}

We estimated the heritability of the apparent nitrogen efficiency and its genetic and phenotypic correlation with feed efficiency traits. Apparent nitrogen efficiency had a heritability ranging from 0.21 to 0.27 , which is comparable to the heritability of protein deposition during the same phases (Godinho et al., 2018). The genetic correlation between nitrogen efficiency and ADG, ADFI, and FCR became stronger from starter to grower to finisher phase. We estimated the nitrogen efficiency at the start of a production phase, as animals are most efficient at that timepoint. This was also shown in the decrease of apparent nitrogen efficiency of our pigs when starter, grower and finisher phase values were compared. van der Peet-Schwering et al. (2021) showed that grower-finisher pigs with a high estimated breeding value for protein deposition have a higher apparent nitrogen efficiency $(55.8 \%)$ than pigs with a low estimated breeding value $(52.7 \%)$. These values are similar to our results, as our average apparent nitrogen efficiency of animals in the same age-range was 50.4 to $55.3 \%$. Our results combined with the results of van der Peet-Schwering et al. (2021) indicate that improving nitrogen efficiency of grower-finisher pigs by means of genetic selection is possible. To select pigs for breeding to improve nitrogen efficiency, large scale phenotyping is needed. Using nitrogen balance measurements to determine nitrogen efficiency as common in nutrition studies is very accurate but not suitable for large scale phenotyping. Although the method we applied is less accurate, it is based on readily available data in the pig breeding industry and can be used to indirectly calculate nitrogen efficiency of individual pigs with average values close to values found in nitrogen balance studies. As such, nitrogen efficiency estimated by feed intake and growth patterns in semi-mechanistic models has the potential to be used in pig breeding.

\subsection{Conclusion}

There is substantial variation in the apparent $A A$ and nitrogen efficiency between pigs and the variation in nitrogen efficiency has a genetic background. Diet and sex had a substantial effect on the AA and nitrogen efficiencies, and nitrogen efficiency decreased when the pigs grew older. Nitrogen efficiency had a small positive correlation with ADG in the young animals, but decreased to a strong negative correlation in older animals. Nitrogen efficiency had a negative correlation with FCR and ADFI. Variation in nitrogen and AA efficiencies can be calculated based on feed intake and growth patterns and selection for increased nitrogen efficiency is possible but would result in decreased FCR by reducing ADFI and ADG. 


\subsection{Acknowledgements}

This research was conducted within the EU project Feed-a-Gene, funded by The European Union's H2020 Program under grant agreement no 633531. We also thank Schothorst Feed Research and the Breed4Food partner Topigs Norsvin for providing data.

\subsection{Declaration of interest}

The authors declare no conflict of interest.

\subsection{Ethics statement}

This study was carried out in strict accordance with the recommendations in the European Guidelines for accommodation and care of animals. The protocol was approved by the Animal Care and Use Committee of Schothorst Feed Research, The Netherlands (Protocol Number: AVD 246002015120/132).

\subsection{Software and data repository resources}

None of the data were deposited in an official repository. 



\section{6}

\section{Combining faecal microbiome, serum metabolome, and host genome for phenotypic prediction of feed efficiency in grower-finisher pigs}

L.M.G. Verschuren ${ }^{1,2,3}$, A.J.M. Jansman², J. van Milgen ${ }^{3}$, O. Zemb ${ }^{4}$, M.S. Hedemann ${ }^{5}$, R. Bergsma ${ }^{1}$, M.P.L. Calus $\left.{ }^{6}\right]$

${ }^{1}$ Topigs Norsvin Research Center B.V., P.O. Box 43, 6640 AA Beuningen, The Netherlands; ${ }^{2}$ Wageningen University and Research, Wageningen Livestock Research, P.O. Box 338, 6700 AH Wageningen, Netherlands; ${ }^{3}$ INRAE - Agrocampus Ouest, UMR1348 Pegase, Saint-Gilles, 35590, France; ${ }^{4}$ INRAE - INPT - ENSAT Université de Toulouse, GenPhySE, Castanet-Tolosan, 31326, France; ${ }^{5}$ Deptartment of Animal Science, Aarhus University, Foulum, Denmark; ${ }^{6}$ Wageningen University and Research, Animal Breeding and Genomics, P.O. Box 338, $6700 \mathrm{AH}$ Wageningen, Netherlands

Submitted 


\begin{abstract}
Background: This study used relationships between individuals derived from the faecal microbiome, blood metabolome and animal genome to predict feed efficiency related traits in pigs (i.e., feed intake, body weight gain, feed conversion ratio, residual feed intake, backfat thickness, and loin depth).
\end{abstract}

Results: Data were collected from 765 three-way crossbred male grower-finisher pigs, genotyped at 50k SNPs. Pigs were offered feed ad libitum in a three-phase feeding program with a commercial diet based on wheat, barley and by-products. On the day before slaughter (mean body weight $125 \mathrm{~kg}$ ), individual faecal and blood samples were collected. Faecal samples were sequenced for the 165 hypervariable region of bacteria (V3-V4) to profile the faecal microbiome. Serum samples were analysed with untargeted LC-MS for metabolites, resulting in metabolites with a positive or negative charge. We estimated the proportion of variance in feed efficiency related traits that was explained by variation in the faecal microbiome (m2), blood metabolites (b2), and SNP genotypes (h2). Metabolites in a positive or negative ionization mode gave similar b2 values, so we continued by using metabolites in the positive mode. The $\mathrm{m} 2, \mathrm{~b} 2$, and $\mathrm{h} 2$ estimates ranged from 7 to $52 \%, 10$ to $65 \%$, and 7 to $28 \%$, respectively. Using metabolome, faecal microbiome and genome profiles simultaneously in different combinations showed overlap between variation explained by the three profiles. Using cross-validation, we estimated prediction accuracies based on the microbiome, metabolome, and genome profile of the pigs. Prediction accuracies were highest based on the serum metabolome profile (0.13-0.64), followed by the faecal microbiome profile (0.050.46), and the genome profile (-0.05-0.29). Combining metabolome, faecal microbiome and genome profiles in different combinations increased prediction accuracies only with 0.01 to 0.10 points. Modelling all three profiles simultaneously resulted in the highest prediction accuracy ( $r=0.15-0.65)$, but was only 0.01 to 0.05 points higher than when only using metabolome profiles.

Conclusions: Using the metabolome and faecal microbiome profiles of pigs, in addition to their and genome profile, improves the accuracy of prediction of phenotypes for feed efficiency related traits, but almost the same prediction accuracy could be achieved using blood metabolite profiles only. 


\subsection{Background}

Feed efficiency is an important trait in pig production. After a pig consumes its diet, nutrients are partly digested in the small intestine and the fraction not digested in the small intestine is can be fermented in the large intestine by resident microbiota. Fermentation products are largely absorbed in the large intestine and transferred to the blood circulation or further metabolized in intestinal tissue, similar to nutrients absorbed in the small intestine. Absorbed nutrients and fermentation products reaching the blood circulation can be used in metabolism in organs and tissues of pigs. Metabolites formed during metabolism can be transferred back to the blood and used elsewhere in the body. Metabolites that are not used in the body are excreted via the urine, the gastro-intestinal tract, or to the air as $\mathrm{CO}_{2}$. Thus, we may hypothesize that variation in individual phenotypes for feed efficiency traits is associated with individual differences in blood metabolite profiles, in addition to being associated with differences in faecal microbiota composition as recently observed (Camarinha-Silva et al., 2017; Aliakbari et al., 2021).

The proportion of the phenotypic variance for a complex trait that is explained by variation in faecal microbiota composition is called "microbiability" (Difford et al., 2016). Here, we would like to introduce the term "metabolomability" for the proportion of variance in a phenotype explained by variation in serum metabolite profiles. It has been shown that relationship matrices based on faecal microbiota composition can be used to predict feed efficiency traits in pigs, with accuracies of 0.41 for average daily gain (ADG), 0.33 for feed conversion ratio (FCR) and 0.33 for average daily feed intake (ADFI) (Camarinha-Silva et al., 2017). Phenotypic predictions based on faecal microbiota profiles were more accurate than those based on pig genomic profiles (Camarinha-Silva et al., 2017), which indicates that faecal microbiota profiles at least partly explain non-heritable variation in phenotypes. A similar method using a blood plasma metabolite relationship matrix was used by Sarup et al. (2018), who found prediction accuracies of 0.68 for total feed intake, 0.59 for ADG and 0.59 for back fat thickness (BF). Using genomic and metabolite information simultaneously resulted in slightly higher prediction accuracies (Sarup et al., 2018). The host genome somehow influences microbiota and blood metabolites, as abundance of several bacterial genera in faeces is heritable with values up to 0.57 (Estelle et al., 2016; Camarinha-Silva et al., 2017), and several blood metabolites are heritable with values up to 0.68 (Casellas et al., 2010; Larzul et al., 2015; Bovo et al., 2019)\}. In addition, genomic regions have been identified in the pig genome that are associated with individual differences in blood 
metabolites (Bovo et al., 2019) and the observed relative abundance for several bacterial genera (Crespo-Piazuelo et al., 2019). However, the interplay between host genome, faecal microbiota composition and blood metabolites in relation to feed efficiency is still unknown.

This study investigated the contribution of host genome, faecal microbiota composition, and concentrations of serum metabolites to variation in feed efficiency traits. The specific aims were (i) to identify the proportion of phenotypic variance in feed efficiency traits associated with microbiota and metabolite profiles, (ii) to evaluate the accuracy of prediction of feed efficiency phenotypes using microbiota and metabolite profiles, and (iii) to estimate the heritability of faecal microbiota and serum metabolites in order to investigate their relationship with the host genome. To achieve these goals, we computed relationship matrices based on genomic, faecal microbiota composition and blood metabolome profiles and used those in mixed model equations to model individual feed efficiency data of $\sim 530$ grower-finisher pigs.

\subsection{Methods}

\subsubsection{Animals and experimental design}

Pigs used in this study originated from a three-way cross (Synthetic boar $x$ (Landrace $x$ Large White)). Phenotypic data were available for 765 male three-breed cross pigs. All pigs were kept under commercial conditions in one farm. The pigs included in the experiment at 9 to 16 weeks of age (Day 0) in 32 groups with 1 - 20 days between consecutive groups. Up until the start of the trial the animals were housed per litter. Pigs were distributed as follows: ten pigs per pen and eight pens per compartment; one compartment was used per entrance date. At the start of the experiment, pigs had an average body weight (BW) of $32( \pm 7.1) \mathrm{kg}$ and were kept in the facilities until they reached a live weight at slaughter of approximately $125 \mathrm{~kg}$. Pigs were allowed a minimum space of $1 \mathrm{~m}^{2}$ per pig, and the pens were equipped with $60 \%$ concrete floor and $40 \%$ slatted floor. The pigs were offered feed ad libitum according to a three-phase feeding program during the growing-finishing phase, in which the diets were commercial diets based on wheat, barley, and by-products, as typically fed in Europe. Only the finishing diet was a custom-made diet, in which minimum and maximum inclusion levels for raw materials were set (Table 6.1). The diets were formulated on a fixed net energy content and ratio of net energy to digestible lysine ( $0.81 \mathrm{~g} / \mathrm{MJ}$ apparent ileal digestible lysine/Net energy), and to meet or exceed the 
nutritional requirements of the animals. The experimental diets were produced in the feed plant of ForFarmers, Lochem, The Netherlands.

Table 6.4 Raw material inclusion levels (in \%) of the diets fed, as-fed basis.

\begin{tabular}{lll}
\hline Raw material & Minimum & Maximum \\
\hline Wheat & 39.2 & 45.0 \\
Barley & 12.8 & 29.0 \\
Palm kernel meal & 3.0 & 6.0 \\
Rapeseed meal & 10.6 & 12.0 \\
Barley starch & 5.0 & 5.0 \\
Sunflower meal & 2.0 & 6.8 \\
Soybean meal & 1.0 & 1.3 \\
Corn & 0.0 & 11.6 \\
Molasses & 1.0 & 3.0 \\
\hline
\end{tabular}

\subsubsection{Measurements and sampling}

The facilities were equipped with IVOG feeding stations (INSENTEC, Marknesse, The Netherlands) that register individual feed intake of group housed animals. All animals had ear tags with unique incremental numbering and individual feed intake records were available for all pigs for each day on test. Animals were weighted at day 0 and at the end of the test. One day before slaughter, individual blood samples were collected from the vena jugularis of 742 pigs, processed to serum and stored at $-80^{\circ} \mathrm{C}$. At the same day as blood sample collection, individual faecal grab samples were collected immediately at defecation. Faecal samples destined for microbiota analysis were collected of 745 pigs, immediately frozen in liquid nitrogen and stored at $-80^{\circ} \mathrm{C}$. In addition, hair samples of 639 animals were taken and used to genotype with 50K SNP chips. At slaughter, measurements of BF and loin depth (LD) were recorded. The ADFI was calculated as the cumulated individual feed intake records throughout the trial divided by the duration of the study in days. The ADG was calculated as the difference between BW measurements divided by the duration of the trial. The FCR was calculated as the ADFI divided by the ADG. The feed intake independent of growth and composition, called residual feed intake (RFI), was obtained as the residual term of the regression (Cai et al., 2008):

$A D F I=\mu+b_{1} B W_{\text {on }}+b_{2} B W_{\text {off }}+b_{3} B F+b_{4} A D G+b_{5}$ age $+e$ in which $A D F I, B F$, and $A D G$ are described previously, $\mu$ is the mean ADFI of the pigs, $B W_{o n}$ is the $B W$ at the start of the trial, $B W_{\text {off }}$ is the $B W$ at the end of the trial, age is the age at the start of the trial, $b_{1}, b_{2}, b_{3}, b_{4}$, and $b_{5}$ are the linear coefficients of the 
6 Microbiome, metabolome, genome and feed efficiency in pigs

regression on covariates, and $e$ is the RFI. Table 6.2 shows an overview of the feed efficiency traits measured.

Table 6.2 Overview of (variation in) feed efficiency traits across animals

\begin{tabular}{llll}
\hline Trait $^{1}$ & Average & Standard deviation & Number of records \\
\hline ADG $(\mathrm{g} / \mathrm{d})$ & 961 & 127 & 744 \\
$\mathrm{ADFI}(\mathrm{g} / \mathrm{d})$ & 2236 & 228 & 740 \\
$\mathrm{FCR}(\mathrm{g} / \mathrm{g})$ & 2.35 & 0.23 & 740 \\
$\mathrm{RFI}(\mathrm{g} / \mathrm{d})$ & 0 & 136 & 618 \\
$\mathrm{BF}(\mathrm{mm})$ & 12.0 & 2.2 & 618 \\
$\mathrm{LD}(\mathrm{mm})$ & 62.2 & 7.1 & 602 \\
\hline
\end{tabular}

${ }^{1} \mathrm{ADG}$, average daily gain; ADFI, average daily feed intake; FCR, feed conversion ratio; RFI, residual feed intake; $B F$, back fat thickness; $L D$, loin depth.

\subsubsection{Faecal microbiota analysis}

For each sample the faecal microbiome was profiled by sequencing the $16 \mathrm{~S}$ hypervariable region of bacteria. For microbial DNA extraction a standardized protocol was used and an optical density measurement to check the quality was performed on the Nanodrop (Agilent Technologies). PCR was used to amplify the $16 \mathrm{~S}$ rDNA V3-V4 fragment using forward primer 343F (5'CTTTCCCTACACGACGCTCTTCCGATCTACGGRAGGCAGCAG) and reverse primer 784R (5'-GGAGTTCAGACGTGTGCTCTTCCGATCTTACCAGGGTATCTAATCCT). Whereby the following PCR conditions were used: $1 \mathrm{~min}$ at $94^{\circ} \mathrm{C}, 30 \times\left(60 \mathrm{~s}\right.$ at $94^{\circ} \mathrm{C}, 60 \mathrm{~s}$ at $65^{\circ} \mathrm{C}, 60$ $\mathrm{S}$ at $\left.72^{\circ} \mathrm{C}\right), 10 \mathrm{~min}$ at $72^{\circ} \mathrm{C}$. Subsequently samples were sequenced by targetedamplicon $16 \mathrm{~S}$ sequencing using the MiSeq sequencer (Illumina) and analysed for taxonomy profile per sample with clustering using the open-source software pipeline DADA2 v1.14.1 R (Callahan et al., 2016) combined with the Silva database v132 in the Rv3.6.3 environment. This resulted in a total of 13691 operational taxonomic units (OTUs). To get taxonomic information, sequences representative for every OTU were aligned against the rRNA gene databases provided by the SILVA ribosomal RNA project (Quast et al., 2013) by using the SILVA Incremental Aligner (SINA) software (Pruesse et al., 2012). Default SINA settings were used to assign the taxonomy of each OTU, with the minimum identity with query sequence set at 0.97 and number of neighbours per query sequence set at ten. For the following steps, relative abundance of OTUs was calculated and OTUs with average relative abundance 
smaller than $0.001 \%$ and present in less than $5 \%$ of the animals were discarded. In total 2260 OTUs were kept. Figure 1 shows an overview of the OTU dataset.
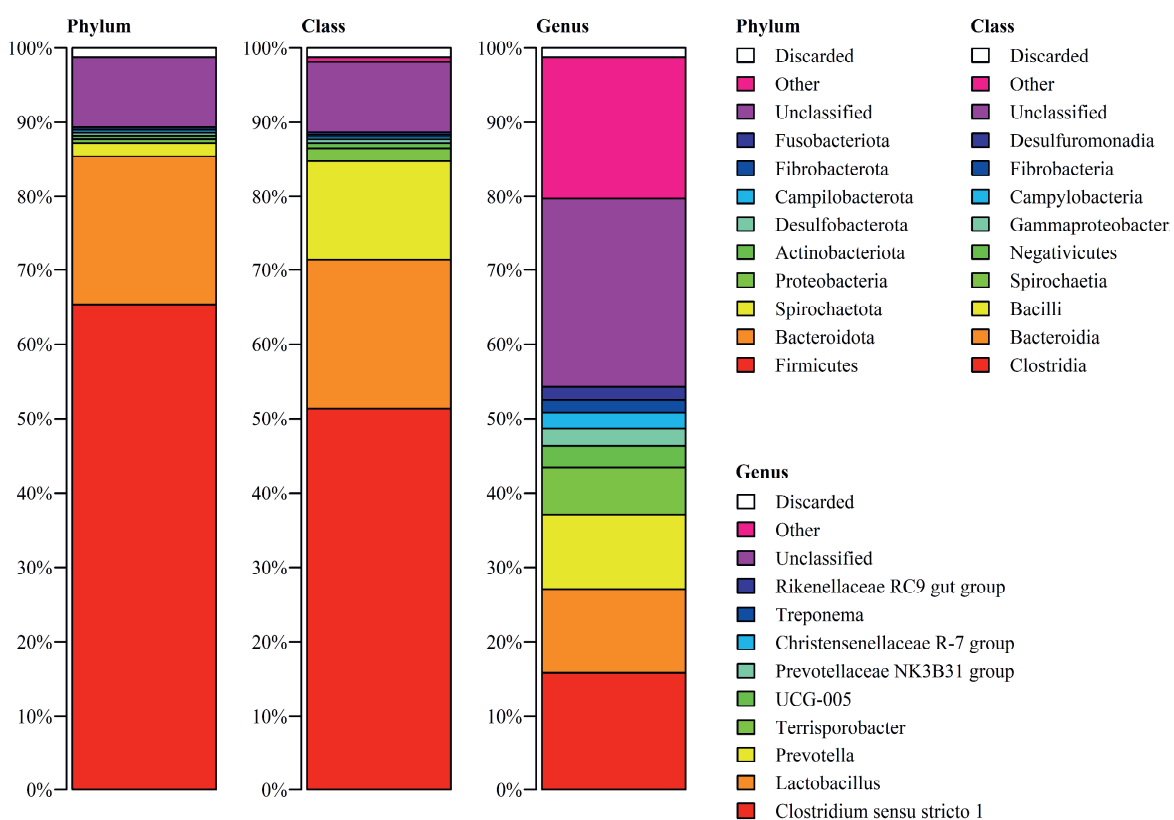

Figure 6.1 Relative abundance of 10 major bacterial phyla, classes and genera in the faeces of pigs used in the present study. Data are mean percentage of total identified sequences.

\subsubsection{Serum metabolite analysis}

Metabolites in the serum were analysed by untargeted liquid chromatography-mass spectrometry (LC-MS) in positive and negative ionisation mode. Serum samples were prepared and analysed as previously described (van der Peet-Schwering et al., 2021). The mass features of the serum samples were extracted using the XCMS package in $\mathrm{R}$ (Smith et al., 2006) after conversion of the mass spectra to the $\mathrm{m} / \mathrm{z} X M L$-format. Peak identification was performed using the "centWave" method and retention time aligned using "Obiwarp" (Smith et al., 2006); missing values were substituted using the "fillPeaks" method (Smith et al., 2006); adducts, fragments, and isotopes were annotated using CAMERA (Kuhl et al., 2012). Exported data tables were filtered to eliminate features present in the control samples called blanks, containing only water, or blinds, containing the solution added to the experimental samples. Retention times were truncated to contain only portions with informative 
chromatographic peaks, and peaks with a coefficient of variation in quality control samples, which is an aggregation of all samples, of more than $35 \%$ were removed to control outlier peaks in individual experimental samples. All processing resulted in a total of 1733 remaining peaks in the positive mode and 938 remaining peaks in the negative mode. Peaks were also grouped based on the CAMERA annotation (Kuhl et al., 2012), resulting in 374 and 195 grouped peaks in the positive and negative mode. These groups were in subsequent analyses represented by either the metabolite with highest number of peaks across the dataset or the mean intensity of all metabolites per group. The peaks were normalized within batch using the QC samples and the Van der Kloet procedure (Van Der Kloet et al., 2009). An initial principal component analysis (PCA) was performed to eliminate potential outliers. In Table 6.3 the ten metabolites with the highest mean intensity are presented.

Table 6.3 List of 10 serum metabolites with highest mean intensity in the positive mode

\begin{tabular}{|c|c|c|c|c|c|}
\hline Metabolite & $\mathrm{RT}^{1}(\min )$ & M-to-Z ratio 2 & $\begin{array}{l}\text { Level } \\
\text { of } \mathrm{id}^{3}\end{array}$ & $\begin{array}{l}\text { Average } \\
\text { intensity }\end{array}$ & $\begin{array}{l}\mathrm{SD}^{4} \\
\text { intensity }\end{array}$ \\
\hline Glycolic acid ${ }^{5}$ & 3.309 & 200.0476 & 1 & 3578543 & 112417 \\
\hline Glycolic acid fragment ${ }^{5}$ & 3.309 & 154.0421 & 1 & 3125221 & 103308 \\
\hline p-chlorophenylalanine ${ }^{5}$ & 6.695 & 467.3206 & 1 & 2111824 & 84451 \\
\hline Phenylalanine fragment & 2.158 & 120.0811 & 4 & 2092415 & 341161 \\
\hline Indoleacrylic acid & 2.914 & 188.0710 & 4 & 2063010 & 438272 \\
\hline Betaine & 0.711 & 118.0865 & 4 & 1857921 & 281603 \\
\hline LysoPC(0:0/16:0) & or & & & & \\
\hline LysoPC(16:0/0:0) & 9.720 & 496.3410 & 4 & 1842655 & 382028 \\
\hline L-Phenylalanine & 2.158 & 166.0866 & 4 & 1497690 & 245389 \\
\hline Piperidine & 1.300 & 86.0966 & 4 & 1493189 & 205632 \\
\hline LysoPC(0:0/18:2(9Z,12Z)) & or & & & & \\
\hline LysoPC(18:2(9z,12Z)/0:0) & 9.307 & 520.3409 & 4 & 1482932 & 389844 \\
\hline
\end{tabular}

${ }^{1}$ Retention time.

${ }^{2}$ Mass-to-charge ratio.

${ }^{3}$ Level of identification: Identified metabolites (level 1), putatively annotated compounds (level 2), putatively characterized compound classes (level 3), and unknown compounds (level 4).

${ }^{4}$ Standard deviation.

${ }^{5}$ internal standard. 


\subsubsection{Estimation of variances}

The phenotypic variance for feed efficiency traits was partitioned into different sources, using microbial relationship matrices (Ross et al., 2013), metabolite relationship matrices, and genomic relationship matrices. The number of records available for the variance estimation was reduced to 530 records for ADG, ADFI, FCR and RFI, and 452 records for BF and LD due to quality control and filtering. The following mixed models were used for the estimation of the microbiability (1.1), metabolomability (1.2), and heritability (1.3) together with the proportion of the variance explained by the common litter effect:

$y=X \beta+Z m+U c+e$,

$y=X \beta+W b+U c+e$,

$y=X \beta+Q g+U c+e$,

where $y$ is the vector of phenotypes (one record per sample), $X$ is the incidence matrix for the fixed effects for pen and co-variable $B W$ at start of the experiment (ADG, ADFI, FCR, RFI) or weight at slaughter (BF, LD), and $\beta$ contains the fixed effects for pen and the regression coefficient for $B W$ at start of the experiment or weight at slaughter. $Z$ is the incidence matrix for OTU effects, $m$ contains the random animal effects associated with OTUs $\sim N\left(0, \mathrm{M \sigma}^{2}{ }_{\mathrm{m}}\right)$, and $\mathrm{M}$ is the microbial relationship matrix. $W$ is the incidence matrix for metabolite effects, $b$ contains the random animal effects associated with metabolites $\sim N\left(0, B \sigma^{2}{ }_{b}\right)$, and $B$ is the metabolite relationship matrix. $Q$ is the incidence matrix for genotype effects, $g$ contains the random animal effects associated with their genome (i.e. genomic breeding values) $\sim N\left(0, G \sigma^{2}\right)$, and $\mathrm{G}$ is the genomic relationship matrix. $U$ is the incidence matrix for common litter effect (foster dam in case of cross-fostering), and c contains the random effects for common litter $\sim N\left(0,1 \sigma^{2}{ }_{c}\right)$. e is the random residuals estimate $N\left(0,1 \sigma^{2}\right.$ e). ASReml (Gilmour et al., 2015) was used to simultaneously estimate, e.g. for model 1.1, $\hat{\sigma}_{m}^{2}, \hat{\sigma}_{c}^{2}, \widehat{\mathbf{m}}$ and $\hat{\boldsymbol{c}}$ from the following equation:

$\left[\begin{array}{ccc}\mathbf{X}^{\prime} \mathbf{X} & \mathbf{X}^{\prime} \mathbf{Z} & \mathbf{X}^{\prime} \mathbf{U} \\ \mathbf{Z}^{\prime} \mathbf{X} & \mathbf{Z}^{\prime} \mathbf{Z}+\mathbf{M}^{-\mathbf{1}} \frac{\boldsymbol{\sigma}^{2}{ }_{e}}{\boldsymbol{\sigma}^{2}{ }_{m}} & \mathbf{Z}^{\prime} \mathbf{U} \\ \mathbf{U}^{\prime} \mathbf{X} & \mathbf{U}^{\prime} \mathbf{Z} & \mathbf{U}^{\prime} \mathbf{U}+\mathbf{I} \frac{\boldsymbol{\sigma}^{2}{ }_{e}}{\boldsymbol{\sigma}^{2}{ }_{c}}\end{array}\right]\left[\begin{array}{c}\boldsymbol{\beta} \\ \mathbf{m} \\ \mathbf{c}\end{array}\right]=\left[\begin{array}{c}\mathbf{X}^{\prime} \mathbf{y} \\ \mathbf{Z}^{\prime} \mathbf{y} \\ \mathbf{U}^{\prime} \mathbf{y}\end{array}\right]$

Solving the equations results in estimates for the fixed effects $(\widehat{\boldsymbol{\beta}})$ as well as the effect for each microbiota profile, such that $\widehat{\mathbf{m}}$ has the dimensions $n \times 1$. A similar model was used to estimate $\hat{\mathbf{b}}$, and $\hat{\mathbf{g}}$. The microbiability was estimated as $\widehat{m}^{2}=$ $\hat{\sigma}_{m}^{2} /\left(\hat{\sigma}_{m}^{2}+\hat{\sigma}_{c}^{2}+\hat{\sigma}_{e}^{2}\right)$ (Difford et al., 2016), metabolomability as $\hat{b}^{2}=\hat{\sigma}_{b}^{2} /\left(\hat{\sigma}_{b}^{2}+\right.$ $\left.\hat{\sigma}_{c}^{2}+\hat{\sigma}_{e}^{2}\right)$, heritability as $\hat{h}^{2}=\hat{\sigma}_{g}^{2} /\left(\hat{\sigma}_{g}^{2}+\hat{\sigma}_{c}^{2}+\hat{\sigma}_{e}^{2}\right)$, and the proportion of variance 
due to common litter effect as $\hat{c}^{2}=\hat{\sigma}_{c}^{2} /\left(\hat{\sigma}_{i}^{2}+\hat{\sigma}_{c}^{2}+\hat{\sigma}_{e}^{2}\right)$ with $i$ being $m, b$, or $g$, for models 1.1, 1.2. and 1.3, respectively.

The microbiota relationship matrix $M$ was computed based on microbiota profiles (matrix Z $[n \times m]$ ), with $n$ samples and $m$ OTUs. Relative abundances were first log transformed, and thereafter centred and scaled before entering in matrix $Z$ (Ross et al., 2013; Camarinha-Silva et al., 2017). Subsequently, a microbial relation matrix was computed based on these microbiota profiles as: $M=Z Z^{\prime} / m$. The metabolite relationship matrix $B$ was computed similarly, but based om metabolite profiles, as $\mathrm{B}=\mathrm{WW}^{\prime} / m$, where $\mathrm{W}$ is a $n \times b$ matrix with $n$ samples and $b$ metabolite peaks. Entries in matrix $W$ reflected centred and standardized intensities for each peak. The genomic relationship matrix $G$ was computed following the first method of VanRaden (2008).

To investigate whether effects on feed efficiency traits of the microbiota, metabolite and genomic profiles were related, additionally a model was implemented that included the microbiota and genomic profiles:

$y=X \beta+Z m+Q g+U c+e$,

or metabolite profiles and genomic profiles:

$y=X \beta+W b+Q g+U c+e$,

or microbiota profiles and metabolite profiles:

$y=X \beta+Z m+W b+U c+e$,

or microbiota, metabolite, and genomic profiles:

$y=X \beta+Z m+W b+Q g+U c+e$,

where $y, X, \beta, Z, m, Q, g, W, b, U, c$ and e are as described for model 1.1 to 1.3.

Significance of each of the random effects was tested by means of the log-likelihood ratio test, using the test statistic $D=2\left[\log \left(L_{2}\right)-\log \left(L_{1}\right)\right]$, where $L_{2}$ is the likelihood of the model including a specific random effect and $L_{1}$ the likelihood of the model excluding this random effect, as provided by ASReml (Gilmour et al., 2015). This means that model 2.1 was compared with model 1.1 to test the significance of the genomic effect, and with model 1.3 to test the significance of the random effect of the microbiota profiles, etc. Likewise, models 1.1 to 1.3 were compared to model 4 that included only a common litter effect:

$y=X \beta+U c+e$,

where $y, X, \beta, U, C$, and e are as described for models 1.1 to 1.3. The distribution of the $D$ test statistics is a mixture of two Chi-square distributions with 0 and 1 degrees of freedom (Self and Liang, 1987). Considering this, the $D$ test statistics were used to calculate $P$-values, where values $<0.05$ were considered to be significant. Model 4 was also used to estimate heritability values for individual OTU and metabolites. 


\subsubsection{Phenotypic prediction}

The prediction accuracy of the aforementioned models was assessed using crossvalidation based on pre-corrected phenotypes. Pre-corrected phenotypes $\mathrm{y}^{*}$ (corrected for fixed effects pen, co-variable BW at start of the experiment (ADG, $A D F I, F C R, R F I)$ or weight at slaughter (BF, LD), and for the random common litter effects) were computed per animal as the sum of its residual and its estimated effects of the tested profiles, obtained from model 3. So, $y^{*}=\widehat{\mathbf{m}}+\hat{\mathbf{b}}+\hat{\mathbf{g}}+\hat{\mathbf{e}}$. In each of the cross-validation folds, the pre-corrected phenotype of the individuals in one pen was removed, and predicted using the individuals in all other 48 pens. In this way, a prediction for each of the individuals was obtained, based on the feed efficiency trait values of all other animals in the data and all microbiota, metabolite, or genomic profiles. The models used for the cross-validation of microbiota profiles were:

$\mathrm{y}^{*}=1_{\mathrm{n}} \mu+Z \mathrm{~m}+\mathrm{e}$

$\mathrm{y}^{*}=1_{\mathrm{n}} \mu+\mathrm{Zm}+\mathrm{Wb}+\mathrm{Qg}+\mathrm{e}$,

where $1_{n}$, is a vector of ones, $\mu$ is the overall mean, $Z, m, W, b, Q, g$, and e are as described in models 1.1 to 1.3 , and the predicted phenotype for the feed efficiency traits obtained from model (5) are: $\hat{\mathbf{y}}^{*}=1_{n} \hat{\mu}+\widehat{\mathbf{m}}$, and from model (6) are: $\hat{\mathbf{y}}^{*}=\mathbf{1}_{\mathbf{n}} \hat{\mu}$ $+\widehat{\mathbf{m}}+\widehat{\mathbf{b}}+\hat{\mathbf{g}}$. The accuracy of these predicted phenotypes $\hat{\mathbf{y}}^{*}$ was computed as the correlation between them and the observed pre-corrected phenotypes $y^{*}$ obtained from model (3). In addition, $\mathrm{y}^{*}$ was regressed on $\hat{\mathbf{y}}^{*}$ to evaluate bias of the predictions. Whether or not the accuracy was significantly different from 0 , was assessed by obtaining the distribution of the accuracy using 10,000 bootstrap samples to recompute the accuracy. Using the described process, predicted feed efficiency trait values were generated and assessed using only microbiota, metabolite or genomic profiles as in model 5 , any of the three pairwise combinations of these profiles, or all three together (model 6).

\subsection{Results}

The comparison between estimated for $b^{2}$ using all or grouped metabolite concentrations in the positive or negative mode are presented in Table 6.4. The $b^{2}$ estimates of all feed efficiency traits based on the positive mode metabolites $\left(b+^{2}\right)$ ranged from 0.09 for $L D$ to 0.65 for $A D G$, whereas the estimates based on the negative mode metabolites $\left(b-{ }^{2}\right)$ ranged from 0.01 for LD to 0.56 for ADFI. The $b^{2}$ estimates were higher for the models using metabolites in the positive mode

Table 6.4 Metabolomability $\left(b^{2}\right)$ for feed efficiency related traits estimated by models using serum metabolites found in the positive $(+)$ and negative (-) mode using all metabolites (Full) 
6 Microbiome, metabolome, genome and feed efficiency in pigs

or the most abundant metabolites in groups of highly correlated metabolites (Cor) or the average of the group of highly correlated metabolites (Mean)

\begin{tabular}{|c|c|c|c|c|c|c|}
\hline \multirow[b]{2}{*}{ Trait $^{1}$} & \multicolumn{3}{|c|}{ Positive OR negative mode } & \multicolumn{3}{|c|}{ Positive AND negative mode } \\
\hline & Full & Cor & Mean & Full & Cor & Mean \\
\hline \multicolumn{7}{|l|}{ ADG } \\
\hline$b++^{2}$ & $0.65^{\ddagger}(0.07)$ & $0.47^{\ddagger}(0.07)$ & $0.52^{\ddagger}(0.06)$ & $0.39^{\ddagger}(0.10)$ & $0.16^{+}(0.07)$ & $0.28^{\ddagger}(0.08)$ \\
\hline$b-{ }^{2}$ & $0.54^{\ddagger}(0.07)$ & $0.47^{\ddagger}(0.07)$ & $0.45^{\ddagger}(0.07)$ & $0.26^{\ddagger}(0.09)$ & $0.36^{\ddagger}(0.08)$ & $0.26^{\ddagger}(0.08)$ \\
\hline \multicolumn{7}{|l|}{ ADFI } \\
\hline$b++^{2}$ & $0.58^{\ddagger}(0.08)$ & $0.41^{\ddagger}(0.07)$ & $0.45^{\ddagger}(0.07)$ & $0.05(0.06)$ & $0.04(0.06)$ & $0.06(0.07)$ \\
\hline$b-^{2}$ & $0.56^{\ddagger}(0.07)$ & $0.41^{\ddagger}(0.07)$ & $0.42^{\ddagger}(0.07)$ & $0.52^{\ddagger}(0.08)$ & $0.38^{\ddagger}(0.07)$ & $0.37^{\ddagger}(0.08)$ \\
\hline \multicolumn{7}{|l|}{ FCR } \\
\hline$b+^{2}$ & $0.51^{\ddagger}(0.08)$ & $0.46^{\ddagger}(0.07)$ & $0.51^{\ddagger}(0.07)$ & $0.27^{+}(0.11)$ & $0.37^{\ddagger}(0.08)$ & $0.45^{\ddagger}(0.08)$ \\
\hline$b-{ }^{2}$ & $0.38^{\ddagger}(0.08)$ & $0.33^{\ddagger}(0.07)$ & $0.32^{\ddagger}(0.07)$ & $0.20^{+}(0.09)$ & $0.10^{+}(0.06)$ & $0.06(0.05)$ \\
\hline \multicolumn{7}{|l|}{ RFI } \\
\hline$b++^{2}$ & $0.38^{\ddagger}(0.09)$ & $0.29^{\ddagger}(0.08)$ & $0.33^{\ddagger}(0.08)$ & $0.00(0.00)$ & $0.06(0.07)$ & $0.07(0.08)$ \\
\hline$b-{ }^{2}$ & $0.38^{\ddagger}(0.09)$ & $0.27^{\ddagger}(0.07)$ & $0.27^{\ddagger}(0.07)$ & $0.38^{\ddagger}(0.09)$ & $0.24^{\ddagger}(0.08)$ & $0.23^{\ddagger}(0.08)$ \\
\hline \multicolumn{7}{|l|}{$\mathrm{BF}$} \\
\hline$b+t^{2}$ & $0.35^{\ddagger}(0.09)$ & $0.31^{\ddagger}(0.08)$ & $0.28^{\ddagger}(0.08)$ & $0.00(0.00)$ & $0.22^{+}(0.09)$ & $0.13(0.09)$ \\
\hline$b-{ }^{2}$ & $0.29^{\ddagger}(0.08)$ & $0.23^{\ddagger}(0.07)$ & $0.25^{\ddagger}(0.07)$ & $0.29^{+}(0.08)$ & $0.10(0.07)$ & $0.16^{+}(0.08)$ \\
\hline \multicolumn{7}{|l|}{ LD } \\
\hline$b+^{2}$ & $0.10^{+}(0.07)$ & $0.09^{+}(0.06)$ & $0.10^{+}(0.06)$ & $0.10^{+}(0.07)$ & $0.09(0.07)$ & $0.10^{+}(0.06)$ \\
\hline$b-^{2}$ & $0.01(0.04)$ & $0.06(0.05)$ & $0.03(0.04)$ & $0.00(0.00)$ & $0.00(0.05)$ & $0.00(0.00)$ \\
\hline
\end{tabular}

${ }^{1} \mathrm{ADG}$, average daily gain; ADFI, average daily feed intake; FCR, feed conversion ratio; RFI, residual feed intake; $B F$, back fat thickness; $L D$, loin depth.

${ }^{+\ddagger}$ Values are significantly different from zero $(P<0.05, P<0.01$, and $P<0.001$, respectively).

compared to the negative mode. The models using either the positive or negative mode had significantly higher $\mathrm{b}^{2}$ estimates when using all compared to either a representative or the mean of the correlated metabolites. The difference between estimates of $b^{2}$ using a representative or the mean of the correlated metabolites was small. When including the information of metabolites in both the positive and negative mode as separate components in the model, $b+^{2}$ and $b-{ }^{2}$ were lower or equal to the $b+^{2}$ and $b-{ }^{2}$ estimates of models with metabolites in only one mode. The total of explained variances by the metabolites in the positive and negative mode combined, i.e. the sum of $b+^{2}$ and $b-^{2}$, was very comparable to the explained variance by the positive mode metabolites only. 
Differences in prediction accuracies between the models using blood metabolites in positive and negative mode were observed (Table 6.5), but were less pronounced than the observed differences in explained variance. Prediction accuracies ranged from 0.02 for LD to 0.67 for ADFI. For all traits, the prediction accuracy was highest for one of the models combining information of the metabolites in positive and negative mode. When only using one mode, the prediction accuracy of the models using the positive mode metabolites was higher for ADG, FCR, BF, and LD, whereas the prediction accuracy of models using negative mode metabolites was higher for ADFI and RFI. However, the difference between the highest and lowest prediction accuracy per feed efficiency trait, ranging from 0.07 for ADG to 0.14 for LD, was relatively small compared to the confidence interval of the prediction accuracy estimates. Most of the slopes of the regression of observed on predicted adjusted phenotypes were very close to 1 , indicating that the scale of almost all predicted phenotypes were unbiased.

Based on the small difference between $b^{2}$ estimates and prediction accuracy values using different serum metabolite models, we choose to continue using positive mode metabolites with the full model (using all peaks) for further analysis combining blood metabolites, faecal microbiota and host genome profiles. The estimates of $\mathrm{m}^{2}$, $\mathrm{b}^{2}$ and $\mathrm{h}^{2}$ of all models are presented in Table 6.6. Models including only one information source resulted in microbiability values across feed efficiency traits of 0.07 to 0.46 , metabolomability values of 0.10 to 0.65 , and heritability values of 0.07 to 0.34. For ADG, ADFI, RFI, and LD metabolomability had the highest values, whereas for FCR and BF microbiability had the highest value. Including both microbiota and genomic information in the model resulted in a somewhat lower $h^{2}$ for ADFI, whereas the $h^{2}$ values of the other traits and all $\mathrm{m}^{2}$ values were relatively unchanged compared to models including only one information source. Including metabolite and genomic information in the model resulted in a lower $\mathrm{h}^{2}$ in all six feed efficiency traits, whereas the $b^{2}$ values did not change much compared to the models including only one source of information. Including microbiota and metabolite information in the model resulted in 0.00 to 0.32 points lower $\mathrm{m}^{2}$ in all the feed efficiency traits compared to the models including only microbiota information. In contrast, the $b^{2}$ values were only slightly lower, 0.01 to 0.11 points, compared to the models including only metabolite information. Combining all three information sources decreased the estimated $\mathrm{m}^{2}, \mathrm{~b}^{2}$, and $\mathrm{h}^{2}$ for all traits except LD. 
6 Microbiome, metabolome, genome and feed efficiency in pigs

Table 6.5 Cross-validation results for feed efficiency related traits predicted by models using serum metabolites found in the positive and negative mode using all metabolites (Full) or the most abundant metabolite in groups of highly correlated metabolites (Cor) or the average of the group of highly correlated metabolites (Mean)

\begin{tabular}{|c|c|c|c|c|c|c|c|c|c|}
\hline \multirow[b]{2}{*}{ Trait $^{1}$} & \multicolumn{3}{|c|}{ Positive mode } & \multicolumn{3}{|c|}{ Negative mode } & \multicolumn{3}{|c|}{ Positive + negative mode } \\
\hline & Full & Cor & Mean & Full & Cor & Mean & Full & Cor & Mean \\
\hline \multicolumn{10}{|l|}{ ADG } \\
\hline Acc & 0.65 & 0.59 & 0.63 & 0.61 & 0.62 & 0.61 & 0.66 & 0.64 & 0.65 \\
\hline $\mathrm{L}-\mathrm{Cl}$ & 0.60 & 0.53 & 0.58 & 0.55 & 0.56 & 0.54 & 0.61 & 0.58 & 0.59 \\
\hline $\mathrm{U}-\mathrm{Cl}$ & 0.69 & 0.64 & 0.68 & 0.67 & 0.67 & 0.66 & 0.71 & 0.69 & 0.70 \\
\hline Slope & 1.02 & 1.00 & 0.99 & 0.99 & 0.97 & 0.97 & 1.02 & 0.98 & 0.99 \\
\hline \multicolumn{10}{|l|}{ ADFI } \\
\hline Acc & 0.60 & 0.54 & 0.57 & 0.67 & 0.64 & 0.64 & 0.67 & 0.64 & 0.64 \\
\hline $\mathrm{L}-\mathrm{Cl}$ & 0.55 & 0.48 & 0.51 & 0.62 & 0.58 & 0.59 & 0.62 & 0.58 & 0.59 \\
\hline $\mathrm{U}-\mathrm{Cl}$ & 0.65 & 0.60 & 0.62 & 0.72 & 0.69 & 0.69 & 0.72 & 0.69 & 0.69 \\
\hline Slope & 1.00 & 0.98 & 0.99 & 1.01 & 0.98 & 0.99 & 1.01 & 0.98 & 0.99 \\
\hline \multicolumn{10}{|l|}{ FCR } \\
\hline Acc & 0.56 & 0.57 & 0.62 & 0.52 & 0.49 & 0.49 & 0.55 & 0.58 & 0.62 \\
\hline $\mathrm{L}-\mathrm{Cl}$ & 0.49 & 0.51 & 0.56 & 0.45 & 0.42 & 0.42 & 0.49 & 0.51 & 0.56 \\
\hline $\mathrm{U}-\mathrm{Cl}$ & 0.62 & 0.63 & 0.67 & 0.59 & 0.56 & 0.56 & 0.61 & 0.64 & 0.67 \\
\hline Slope & 1.02 & 0.99 & 0.99 & 0.99 & 0.96 & 0.96 & 1.02 & 0.98 & 1.00 \\
\hline \multicolumn{10}{|l|}{$\mathrm{RFI}$} \\
\hline Acc & 0.44 & 0.41 & 0.44 & 0.52 & 0.47 & 0.47 & 0.52 & 0.47 & 0.47 \\
\hline $\mathrm{L}-\mathrm{Cl}$ & 0.37 & 0.33 & 0.36 & 0.46 & 0.40 & 0.39 & 0.45 & 0.40 & 0.40 \\
\hline $\mathrm{U}-\mathrm{Cl}$ & 0.51 & 0.49 & 0.51 & 0.59 & 0.54 & 0.54 & 0.59 & 0.54 & 0.55 \\
\hline Slope & 1.02 & 1.06 & 1.03 & 1.00 & 1.02 & 0.98 & 1.00 & 1.04 & 0.98 \\
\hline \multicolumn{10}{|l|}{$\mathrm{BF}$} \\
\hline Acc & 0.44 & 0.48 & 0.45 & 0.46 & 0.45 & 0.46 & 0.46 & 0.49 & 0.47 \\
\hline $\mathrm{L}-\mathrm{Cl}$ & 0.36 & 0.41 & 0.38 & 0.39 & 0.37 & 0.39 & 0.39 & 0.41 & 0.39 \\
\hline $\mathrm{U}-\mathrm{Cl}$ & 0.51 & 0.56 & 0.53 & 0.53 & 0.52 & 0.53 & 0.53 & 0.56 & 0.54 \\
\hline Slope & 1.02 & 1.06 & 1.05 & 1.00 & 1.02 & 1.03 & 1.00 & 1.04 & 1.03 \\
\hline \multicolumn{10}{|l|}{ LD } \\
\hline Acc & 0.15 & 0.14 & 0.16 & 0.02 & 0.06 & 0.02 & 0.15 & 0.13 & 0.16 \\
\hline $\mathrm{L}-\mathrm{Cl}$ & 0.07 & 0.05 & 0.08 & -0.08 & -0.04 & -0.08 & 0.07 & 0.05 & 0.07 \\
\hline $\mathrm{U}-\mathrm{Cl}$ & 0.24 & 0.22 & 0.24 & 0.12 & 0.16 & 0.11 & 0.24 & 0.22 & 0.24 \\
\hline Slope & 1.00 & 1.03 & 0.96 & 1.00 & 0.97 & 0.26 & 1.00 & 0.97 & 0.96 \\
\hline
\end{tabular}




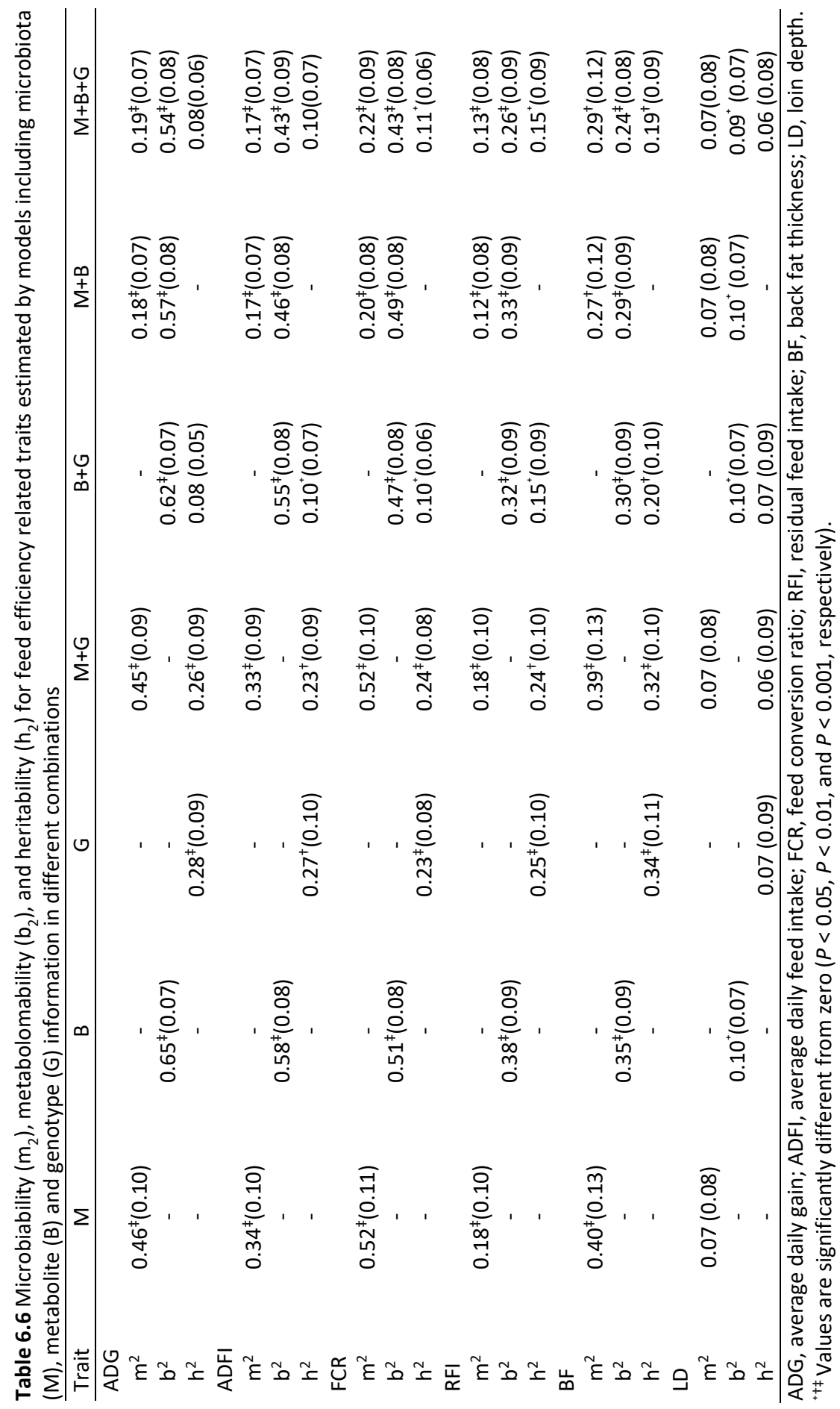


The results of the predictions of feed efficiency traits based on faecal microbiota, blood metabolites or genomic profiles are presented in Table 6.7. The prediction accuracies ranged from 0.05 to 0.46 when using only microbiota relationship matrices, from 0.13 to 0.64 when using only metabolite relationship matrices, and from -0.05 to 0.29 when using only genomic relationship matrices. Combining

Table 6.7 Cross-validation results for feed efficiency traits estimated by models including microbiota, metabolite and genotype information in different combinations

\begin{tabular}{|c|c|c|c|c|c|c|c|c|c|c|}
\hline \multirow{2}{*}{ Trait $^{1}$} & \multirow{2}{*}{$M$} & \multirow{2}{*}{ B } & \multirow{2}{*}{ G } & \multirow{2}{*}{$M+G$} & \multirow{2}{*}{$B+G$} & \multirow{2}{*}{$M+B$} & \multicolumn{4}{|c|}{$M+B+G 2$} \\
\hline & & & & & & & $M+B+G$ & $\mathrm{M}$ & B & G \\
\hline \multicolumn{11}{|l|}{ ADG } \\
\hline Acc & 0.43 & 0.64 & 0.21 & 0.47 & 0.64 & 0.65 & 0.65 & 0.35 & 0.62 & 0.07 \\
\hline $\mathrm{L}-\mathrm{Cl}$ & 0.36 & 0.58 & 0.12 & 0.39 & 0.58 & 0.60 & 0.60 & 0.27 & 0.57 & -0.01 \\
\hline $\mathrm{U}-\mathrm{Cl}$ & 0.50 & 0.68 & 0.29 & 0.53 & 0.68 & 0.70 & 0.70 & 0.43 & 0.67 & 0.17 \\
\hline Slope & 0.99 & 1.01 & 0.86 & 0.97 & 1.02 & 1.00 & 1.00 & 1.62 & 1.12 & 1.36 \\
\hline \multicolumn{11}{|l|}{ ADFI } \\
\hline Acc & 0.46 & 0.59 & 0.25 & 0.50 & 0.60 & 0.62 & 0.63 & 0.43 & 0.55 & 0.13 \\
\hline $\mathrm{L}-\mathrm{Cl}$ & 0.39 & 0.54 & 0.17 & 0.43 & 0.55 & 0.57 & 0.58 & 0.35 & 0.49 & 0.05 \\
\hline $\mathrm{U}-\mathrm{Cl}$ & 0.53 & 0.65 & 0.32 & 0.56 & 0.66 & 0.67 & 0.68 & 0.49 & 0.61 & 0.22 \\
\hline Slope & 1.02 & 1.00 & 0.90 & 1.03 & 1.02 & 0.98 & 0.99 & 1.42 & 1.15 & 2.46 \\
\hline \multicolumn{11}{|l|}{ FCR } \\
\hline Acc & 0.27 & 0.54 & 0.15 & 0.33 & 0.54 & 0.56 & 0.56 & 0.16 & 0.53 & 0.08 \\
\hline $\mathrm{L}-\mathrm{Cl}$ & 0.18 & 0.47 & 0.06 & 0.23 & 0.47 & 0.49 & 0.49 & 0.07 & 0.46 & -0.02 \\
\hline $\mathrm{U}-\mathrm{Cl}$ & 0.36 & 0.60 & 0.24 & 0.41 & 0.60 & 0.62 & 0.62 & 0.24 & 0.59 & 0.17 \\
\hline Slope & 0.85 & 1.03 & 0.80 & 0.85 & 1.01 & 1.00 & 0.98 & 0.76 & 1.03 & 0.79 \\
\hline \multicolumn{11}{|l|}{ RFI } \\
\hline Acc & 0.27 & 0.42 & 0.21 & 0.33 & 0.43 & 0.46 & 0.47 & 0.27 & 0.36 & 0.19 \\
\hline $\mathrm{L}-\mathrm{Cl}$ & 0.17 & 0.34 & 0.12 & 0.24 & 0.35 & 0.38 & 0.39 & 0.07 & 0.46 & -0.02 \\
\hline $\mathrm{U}-\mathrm{Cl}$ & 0.36 & 0.49 & 0.30 & 0.42 & 0.50 & 0.53 & 0.54 & 0.24 & 0.59 & 0.17 \\
\hline Slope & 0.96 & 1.00 & 0.90 & 0.98 & 1.00 & 1.01 & 1.01 & 0.76 & 1.03 & 0.79 \\
\hline \multicolumn{11}{|l|}{ BF } \\
\hline Acc & 0.31 & 0.45 & 0.29 & 0.41 & 0.48 & 0.47 & 0.50 & 0.28 & 0.42 & 0.25 \\
\hline $\mathrm{L}-\mathrm{Cl}$ & 0.22 & 0.37 & 0.20 & 0.33 & 0.40 & 0.39 & 0.42 & 0.19 & 0.34 & 0.15 \\
\hline $\mathrm{U}-\mathrm{Cl}$ & 0.39 & 0.52 & 0.38 & 0.49 & 0.55 & 0.54 & 0.57 & 0.37 & 0.50 & 0.34 \\
\hline Slope & 0.91 & 1.02 & 1.04 & 0.97 & 1.03 & 0.96 & 0.98 & 1.21 & 1.18 & 1.53 \\
\hline \multicolumn{11}{|l|}{ LD } \\
\hline Acc & 0.05 & 0.13 & -0.05 & 0.05 & 0.13 & 0.15 & 0.15 & 0.06 & 0.14 & -0.05 \\
\hline $\mathrm{L}-\mathrm{Cl}$ & -0.04 & 0.05 & -0.16 & -0.05 & 0.05 & 0.06 & 0.06 & -0.04 & 0.05 & -0.16 \\
\hline $\mathrm{U}-\mathrm{Cl}$ & 0.15 & 0.22 & 0.06 & 0.14 & 0.22 & 0.23 & 0.23 & 0.16 & 0.23 & 0.05 \\
\hline Slope & 0.71 & 0.91 & -4.73 & 0.62 & 0.90 & 0.92 & 0.91 & 0.82 & 0.95 & -6.64 \\
\hline
\end{tabular}

${ }^{1} \mathrm{M}$, microbiota information; $B$, metabolite information; $G$, genotype information; ADG, average daily gain; $A D F I$, average daily feed intake; $F C R$, feed conversion ratio; RFI, residual feed intake; BF, back fat thickness; LD, loin depth; Acc, accuracy of prediction; L-Cl, lower bound of the confidence interval; U-CL, upper bound of the confidence interval; Slope, slope of the regression of observed on predicted values.

${ }^{2}$ Estimates given for the total model and for model components. 
microbiota and genomic relationship matrices in the prediction models increased the prediction accuracies up to 0.10 points when compared to models including only one of the two matrices, but combining metabolite and genomic relationship matrices resulted only in a minor increase in prediction accuracy of up to 0.03 points. Combining microbiota and metabolite information matrices resulted in a slight increase in prediction accuracies of up to 0.04 points when compared to using a model with only the metabolite relationship matrix. Using all three relationship matrices, however, did hardly increase the prediction accuracy when compared to using a model including both the microbiota and metabolite relationship matrices, with the difference being highest at 0.03 for BF. Using all three relationship matrices for prediction, we also evaluated the prediction accuracy of the three model components separately. The prediction accuracy based on the metabolite relationship matrix was highest for all feed efficiency traits, ranging from 0.14 to 0.62. Prediction accuracy of the genomic relationship matrix was lowest for all feed efficiency traits, ranging from -0.05 to 0.25 . Finally, the prediction accuracy of the genomic component was always lower compared to the prediction accuracy using genotype information only.

The overlap in predictions based on different information sources is presented in Figure 6.2, where correlations among predicted FCR based on metabolite profiles have a correlation of 0.98 to 1 in all models, independent of the other information sources included in the models. In contrast, the correlations between estimates based on host genotype ranged from 0.84 to 0.98 and based on microbiota profile ranged from 0.60 to 0.99 , depending on the other information sources used in the models. The correlations between the $B$ estimates and $\mathrm{G}$ estimates ranged from 0.07 to 0.17 , whereas the correlations between the $M$ estimates and the $G$ estimates were lower and ranged from -0.09 to 0.07 . The correlation between $M$ and $B$ estimates were the highest when the metabolite profiles were not included in the models estimating $M$, ranging from 0.31 to 0.36 . Including the metabolite profiles in the models decreased the correlations between $M$ and $B$, with estimates ranging from -0.06 to 0.05 . Similar figures for the other feed efficiency traits are presented in Additional files 1 to 5, Figures S1 to S5. The patterns in relationships between $M$, $B$ and $G$ estimates are similar for $A D G, A D F I, R F I$ and $B F$ with only some differences in the relationship between the $M$ and $B$ estimates. The pattern of $L D$ is different from the others, by showing correlations close to 0 for all combinations of $M, B$ and G. The heritability estimates for individual OTUs and metabolites are presented in Figure 6.3. Additional files 6.6 and 6.7, Table 6.S1 and 6.S2, present the heritability and contribution to prediction of feed efficiency traits per OTU and metabolite. Most 
of the OTUs and metabolites have a low heritability, and the average heritability for OTUs was 0.05 and for metabolites 0.08 , but there are some OTU with a heritability up to 0.47 and metabolites with a heritability up to 0.64 .

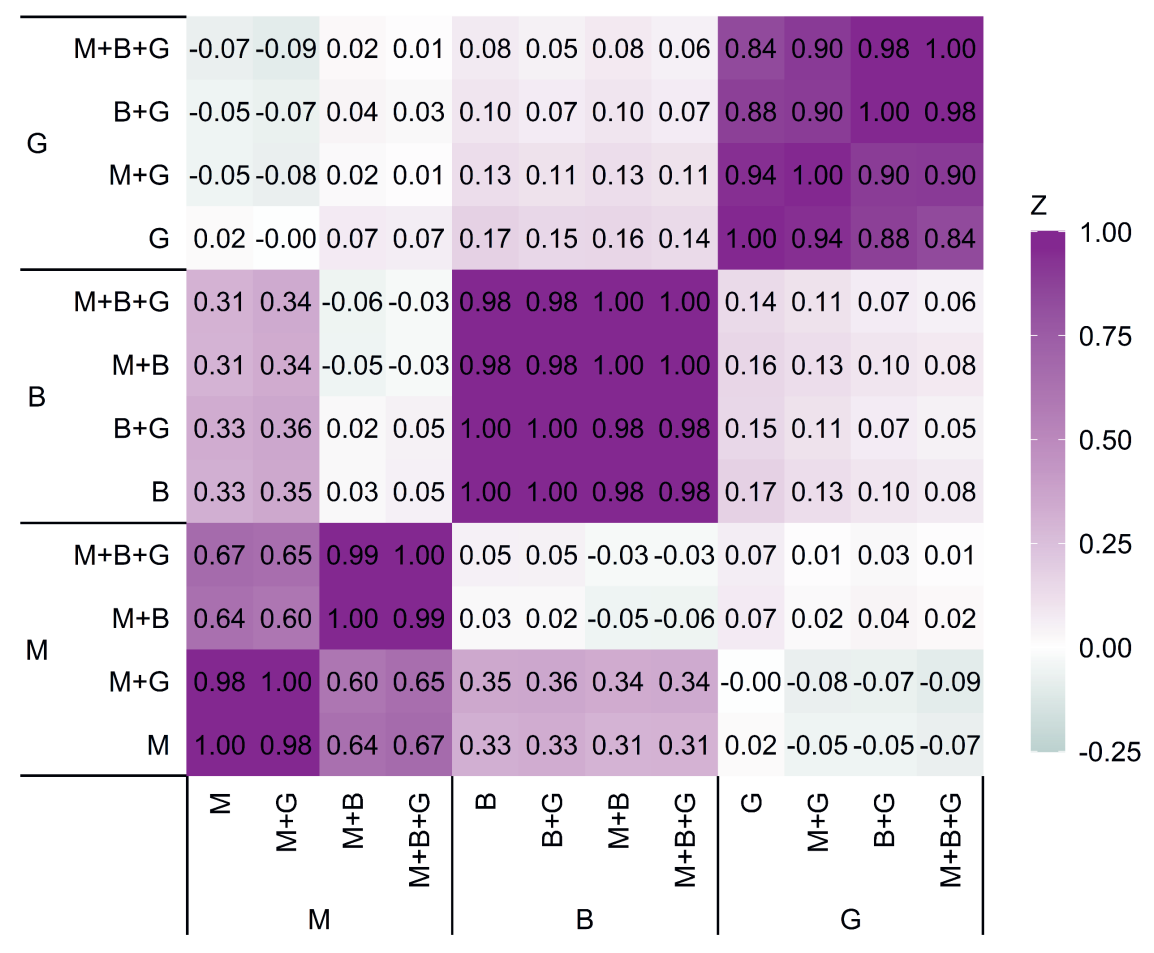

Figure 6.2 Heatmap of correlations between predicted effects of feed conversion ratio by models including microbiota (M), metabolite (B) and genotype (G) information in different combinations. 
OTU

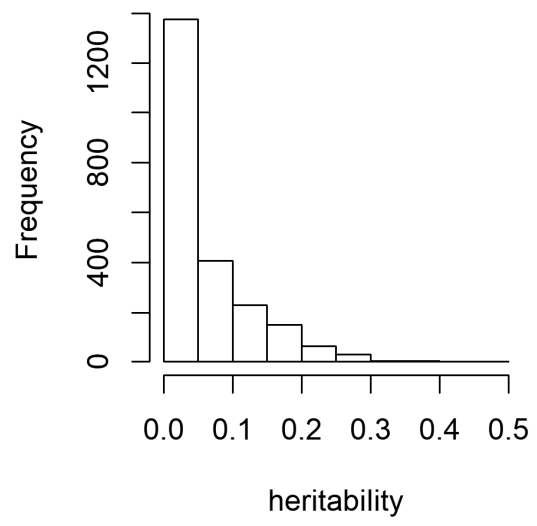

Metabolite

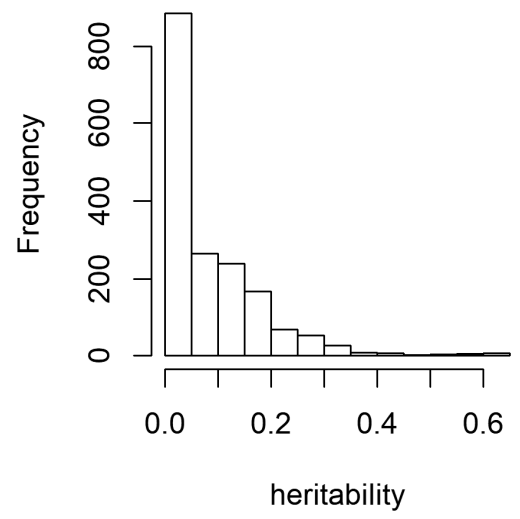

Figure 6.3 Distribution of heritability values of all operational taxonomic units (OTU) and metabolites.

\subsection{Discussion}

\subsubsection{Contribution to phenotypic variation}

This study investigated the contribution of host genome, faecal microbiota composition, and concentrations of blood metabolites to variation in feed efficiency traits. The metabolite profiles produced by LC-MS can result in metabolites measured at the same retention time that are highly correlated. We investigated if using all metabolites produced by the LC-MS would give different results than using the metabolite data after further processing considering the interrelationships between metabolites. Even though the explained variances in the feed efficiency traits were somewhat different when using the processed or the full metabolite data, prediction accuracies were similar. Another processing step we investigated was the ionization mode applied during the mass spectrometry analysis. In principle, several metabolites should be captured by both the positive and negative ionization mode of the mass spectrometer, while several other metabolites likely will be captured only by either mode. Even though the metabolites captured by both ionization modes is only 2 to $6 \%$ (Nordström et al., 2008), the explained variance and prediction accuracy of models combining in the metabolites of both ionization modes did not increase compared to models using one ionization mode only. The results of our study show, that using the metabolites obtained in positive mode resulted in higher explained variances for all feed efficiency traits than using the negative mode 
metabolites, whereas the difference in prediction accuracy was minimal. Hence, to ease implementation and interpretation, we decided to use the full metabolite data measured in positive mode for further analysis.

Serum metabolite concentrations explained most variation in all feed efficiency traits, followed by faecal microbiota composition and host genome. Combining metabolite with microbiota and genotype information resulted in lower metabolomability, microbiability, and heritability values than models with only metabolite, microbiota or genotype information, indicating an overlap between the information sources as observed in Figure 2. Our microbiability values for ADG, ADFI, and FCR are higher than the values predicted by Camarinha-Silva et al. (2017), who estimated values of $0.28,0.16$ and 0.21 respectively. Similarly to our research, these authors reported heritability values lower than microbiability values for the investigated traits (Camarinha-Silva et al., 2017). GWAS results indicate that the faecal microbiota composition is possibly influenced by pig genes related to the expression of mono and polysaccharides in the mucin of the intestinal lining, the adaptive and innate immune response, bile acid and fatty acid metabolism, and intestinal glucose uptake (Crespo-Piazuelo et al., 2019). Combining microbiota and genotype information gave similar results to models with only microbiota or only genotype information, indicating that the microbiota explaining most of the variance in the feed efficiency traits are most likely under limited control of the host genome. Indeed, even though there were OTU with heritability values up to 0.47 , which is comparable to the heritability values found for bacterial genera by (Estelle et al., 2016; Camarinha-Silva et al., 2017), the 10 most important OTU explaining variance in the feed efficiency traits had average heritability values ranging from 0.02 to 0.07 . In contrast, there was substantial overlap between the blood metabolite and the host genome model component of models including either both or a single source of information. GWAS results indicate that genes directly involved in the biological mechanisms of energy or protein metabolism were related to serum metabolite levels (Bovo et al., 2019). The host genetic control of blood metabolite concentration was also visible in the heritability values, where the top 10 contributing metabolites had an average heritability ranging from 0.05 to 0.22 . The heritability value for serum metabolite concentration ranged from 0.00 to 0.64 , with an average of 0.08 across all metabolites. These heritability estimates are similar to the heritability values of 0.27 to 0.47 for several lipid serum metabolites found by (Casellas et al., 2010), the heritability value of 0.68 for cortisol after ACTH stimulation (Larzul et al., 2015) and the heritability values of haematological and blood clinical-biochemical traits ranging from 0.09 to 0.44 (Bovo et al., 2019). The overlap between predictions based on 
microbiota and metabolite information sources was substantial. Part of the bacterial fermentation products end up in the blood of the host and are used in systemic metabolism, whereas some blood metabolites, like urea, are partially transported to the intestines and used by intestinal microbiota as source of fermentation. These biological connections between bacteria and blood metabolites may explain the observed overlap between the variance explained in feed efficiency traits by faecal microbiota composition and blood metabolite concentrations.

\subsubsection{Accuracy of phenotypic prediction}

We aimed to evaluate the accuracy of prediction of feed efficiency phenotypes using combinations of faecal microbiota, serum metabolite and host genome profiles. To estimate the prediction accuracy, we executed a cross-validation on pen level. Faecal microbiota is more similar between pen-mates than pigs in other pens (Verschuren et al., 2020). When allocating animals at random to cross-validation folds, predictions could be partly based on information from pen-mates, while in applications in practice this is unlikely to be the case. Hence, we choose to allocate entire pens to cross-validation folds, such that all animals in the same pen were predicted at the same time. The prediction accuracy using the microbiota relationship matrix was 0.44 for $A D G, 0.48$ for $A D F I$, and 0.30 for $F C R$, which is in line with the prediction accuracy of 0.41 for ADG, 0.33 for ADFI and 0.33 for FCR estimated by Camarinha-Silva et al. (2017). Like our results, Camarinha-Silva et al. (2017) found higher prediction accuracies using a microbiota relationship matrix compared to using a genomic relationship matrix. Combining microbiota with genotype information resulted in a small improvement of the prediction accuracy. The prediction accuracy using the metabolite relationship matrix was 0.63 for ADG and 0.66 for ADFI, which is in line with the prediction accuracy of 0.59 for ADG and 0.68 for total feed intake estimated by Sarup et al. (2018). Combining metabolite with genotype information resulted in a neglectable improvement of prediction accuracy, while Sarup et al. (2018) reported a small increase in prediction accuracy. To our best knowledge, to date no estimates of prediction accuracy using microbiota and metabolite relationship matrices in the same model have been reported. In our study, metabolite relationship matrices were the best in predicting ADG, ADFI, and FCR, somewhat better than microbiota and vastly better than genomic relationship matrices. Although we only had $~ 530$ observations, the slope of the predictions using all three relationship matrices simultaneously was close to 1 , indicating that there was little bias in the predictions. It should be noted that in our study the pigs were housed in one farm, were fed a diet with little variation in ingredient composition, and were sampled at a similar moment, the day before slaughter. An important 
question is how the accuracy would change, if based on our data we would predict phenotypes of pigs housed in a different farm or fed a different diet. Genotypes are stable over the lifetime of a pig, whereas microbiota composition of faecal samples changes with age (Kim et al., 2011; Le Sciellour et al., 2019a) and is largely influenced by environmental factors like diet (Le Sciellour et al., 2018; Verschuren et al., 2018), environmental temperature (Le Sciellour et al., 2019b), and geographic location (McCormack et al., 2019). Maltecca et al. (2019) showed that faecal samples taken at 15 and 22 weeks of age were better for predicting carcass traits in pigs than faecal samples taken at weaning. For serum metabolite concentration the time of measurement may be even more critical, as blood metabolite levels change considerably within a single day (Nielsen et al., 2015). Increasing the variation in the dataset by including data of pigs from different ages, farms, geographical locations and fed different diets, likely would increase the robustness of the prediction equations across environments.

The genomic prediction accuracy of feed efficiency traits was decreased when metabolite and microbiota information were additionally included in the model, so using those information sources for genomic prediction the way we did is not promising. On the other hand, the phenotypic prediction based on microbiota and metabolite information can be very useful for large scale phenotyping of pigs. Even though our study shows that serum metabolite relationship matrices provide the most accurate predictions for feed efficiency traits, collecting serum metabolite data has its challenges. Collecting samples for serum metabolite analysis is more difficult than genotyping pigs, as blood samples need to be drawn, which requires restraining the pig and performing an invasive action. In contrast, genotyping can be done by collecting a couple of hairs or by taking a tissue sample when pigs are tagged. Collecting samples for faecal microbiota data is easier than collecting samples for data on serum metabolites, as fresh faeces can be collected without restraining the pig. Microbiota determination via Illumina Mi-seq in the lab, however, is more labour demanding and time consuming than serum metabolite determination by LC-MS. Over the years, lab processes for genotyping has been scaled-up to routinely handle large numbers of samples, whereas microbiota and metabolite analyses are still in the process of scaling up. For breeding value estimation predicting feed efficiency phenotypes based on faecal microbiota or serum metabolite profiles could be interesting when the cost of blood or faecal sampling and analysis is lower than actually measuring the feed efficiency traits. We depend on technology companies to improve the efficiency of sample processing, making blood metabolite and faecal 
microbiota good candidates for future use in phenotypic prediction of feed efficiency traits for animal breeding and management practices.

\subsection{Conclusions}

Blood metabolite profiles explained the highest proportion of variance of feed efficiency traits, followed by faecal microbiome profile and host genotype. A substantial overlap between phenotypic predictions based on host genotype and blood metabolite profile exists, whereas there was hardly any overlap between linear predictions based on host genotype and faecal microbiome profile. The genomic prediction was more accurate when only using genotype information. The total phenotypic prediction accuracy was highest combining all three information sources, but almost the same prediction accuracy could be achieved using blood metabolite profiles alone. In conclusion, modelling feed efficiency traits as function of genome, microbiome and metabolite profiles improves prediction accuracy of phenotypes, but not of breeding values.

\subsection{Declarations}

Ethics approval and consent to participate

This study was carried out in strict accordance with the recommendations in the European Guidelines for accommodation and care of animals. The protocol was approved by the Central Authority for Scientific Procedures on Animals, The Netherlands (Protocol Number: AVD 230002017821).

\subsection{Consent for publication}

Not applicable.

\subsection{Availability of data and materials}

The data used for this research is not publicly available.

\subsection{Competing interests}

The authors declare that they have no competing interests.

\subsection{Funding}

This research was conducted within the EU project Feed-a-Gene, funded by The European Union's H2020 Program under grant agreement no 633531. 


\subsection{Authors' contributions}

LV, AJ, RB, MC participated in the design of the study. OZ carried out the lab analysis of faecal samples and pre-processing of the microbiome data. HM carried out the lab analysis of blood samples and pre-processing of the metabolome data. LV carried out the data analysis and wrote the manuscript. MC helped to carry out the data analysis. AJ and MC helped to write the manuscript. AJ, JVM, OZ, MH, RB, MC provided helpful comments for writing the manuscript. All authors read and approved the final manuscript.

\subsection{Acknowledgements}

We thank INRAE, Aarhus University and Topigs Norsvin for providing data.

\subsection{Author's information (optional)}

Not applicable.

\subsection{Footnotes}

Not applicable.

\subsection{Additional files}


6 Microbiome, metabolome, genome and feed efficiency in pigs

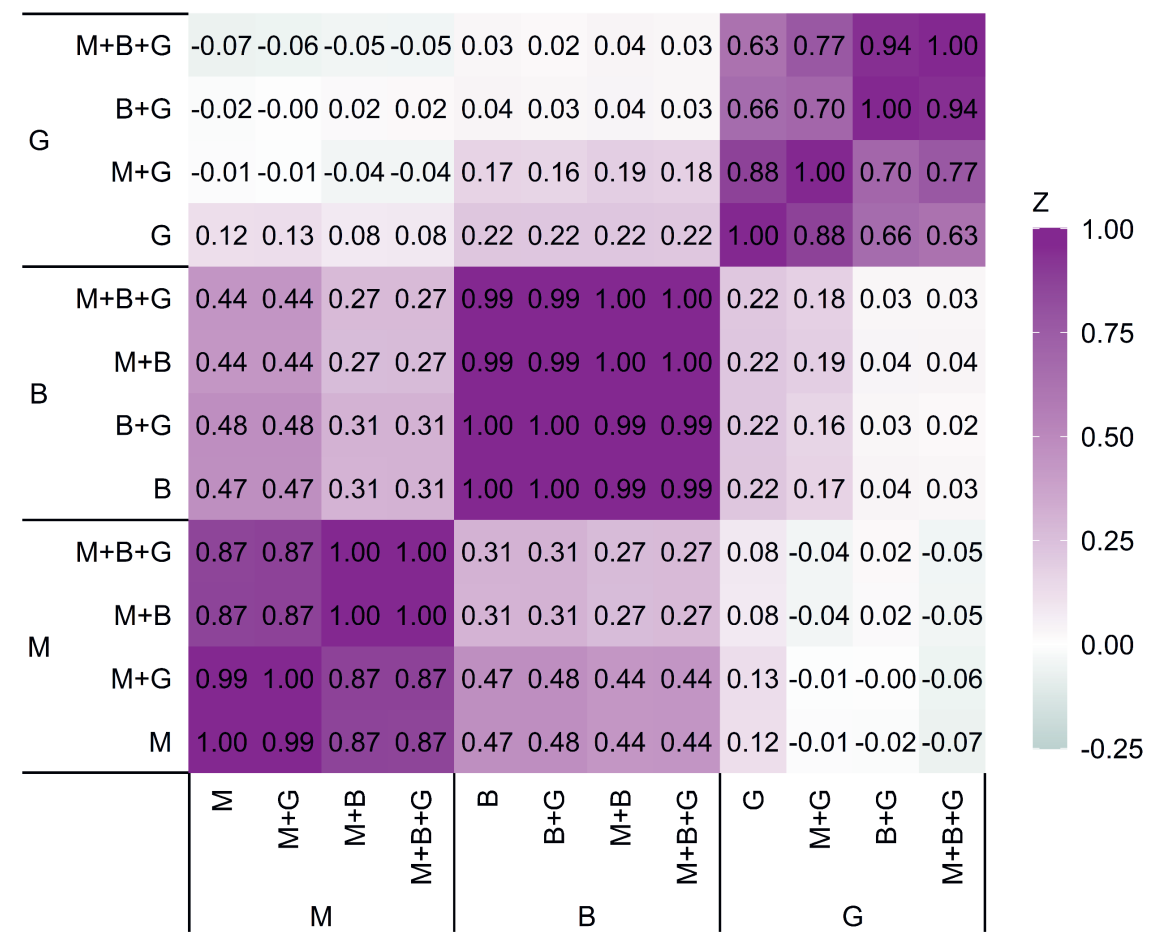

Additional file 6.1 Figure 6.S1 Heatmap of correlations between estimated effects of average daily gain by models including microbiota $(M)$, metabolite $(B)$ and genotype $(G)$ information in different combinations 
6 Microbiome, metabolome, genome and feed efficiency in pigs

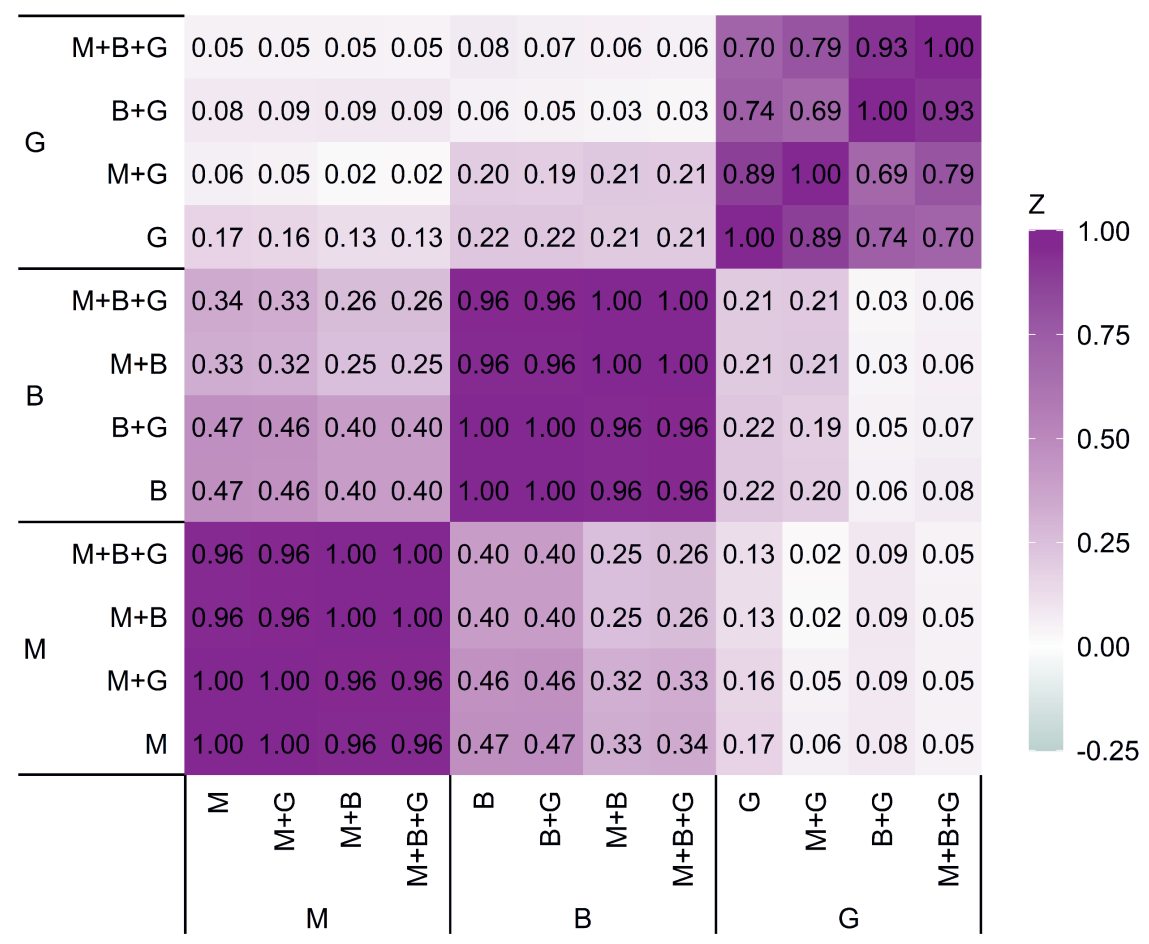

Additional file 6.2 Figure 6.S2 Heatmap of correlations between estimated effects of average daily feed intake by models including microbiota (M), metabolite (B) and genotype (G) information in different combinations 
6 Microbiome, metabolome, genome and feed efficiency in pigs

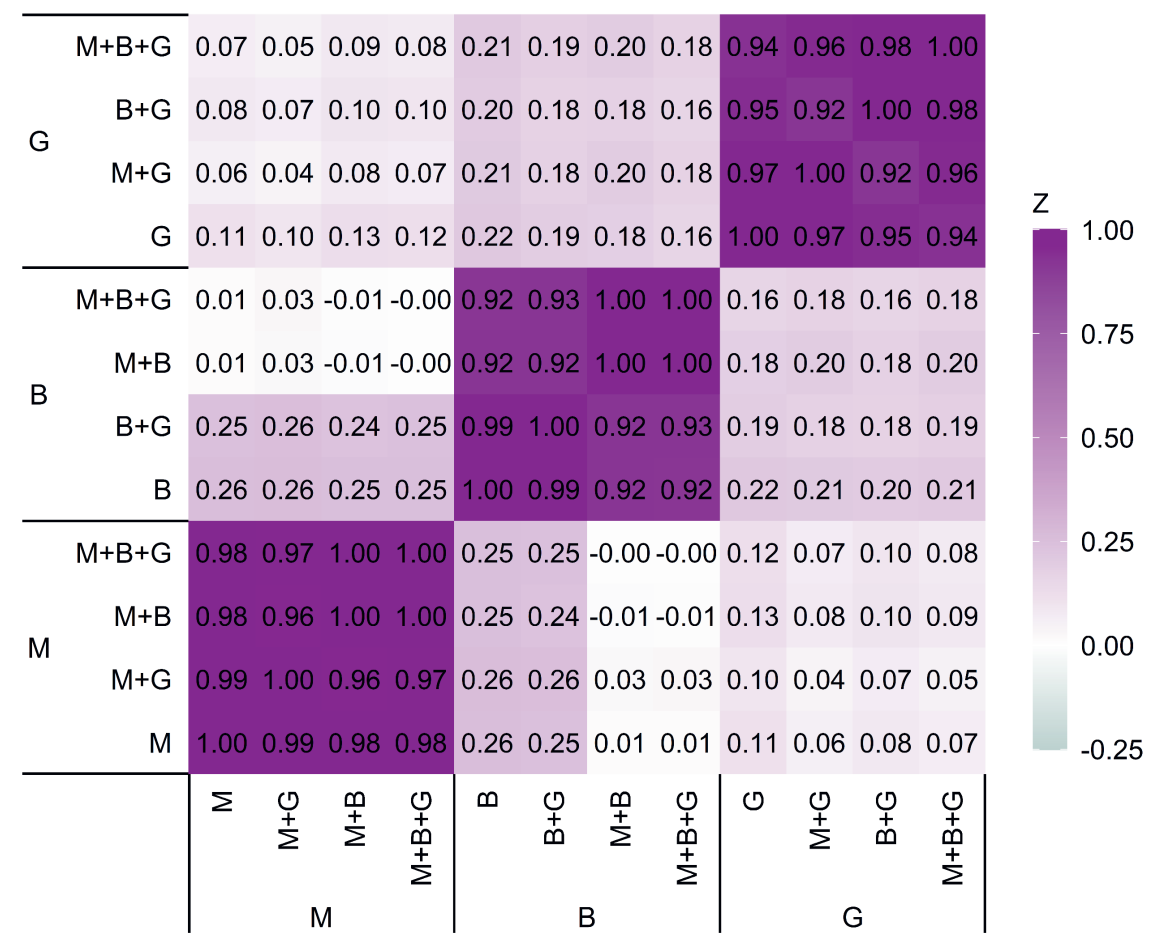

Additional file 6.3 Figure 6.53 Heatmap of correlations between estimated effects of residual feed intake by models including microbiota $(M)$, metabolite $(B)$ and genotype $(G)$ information in different combinations 
6 Microbiome, metabolome, genome and feed efficiency in pigs

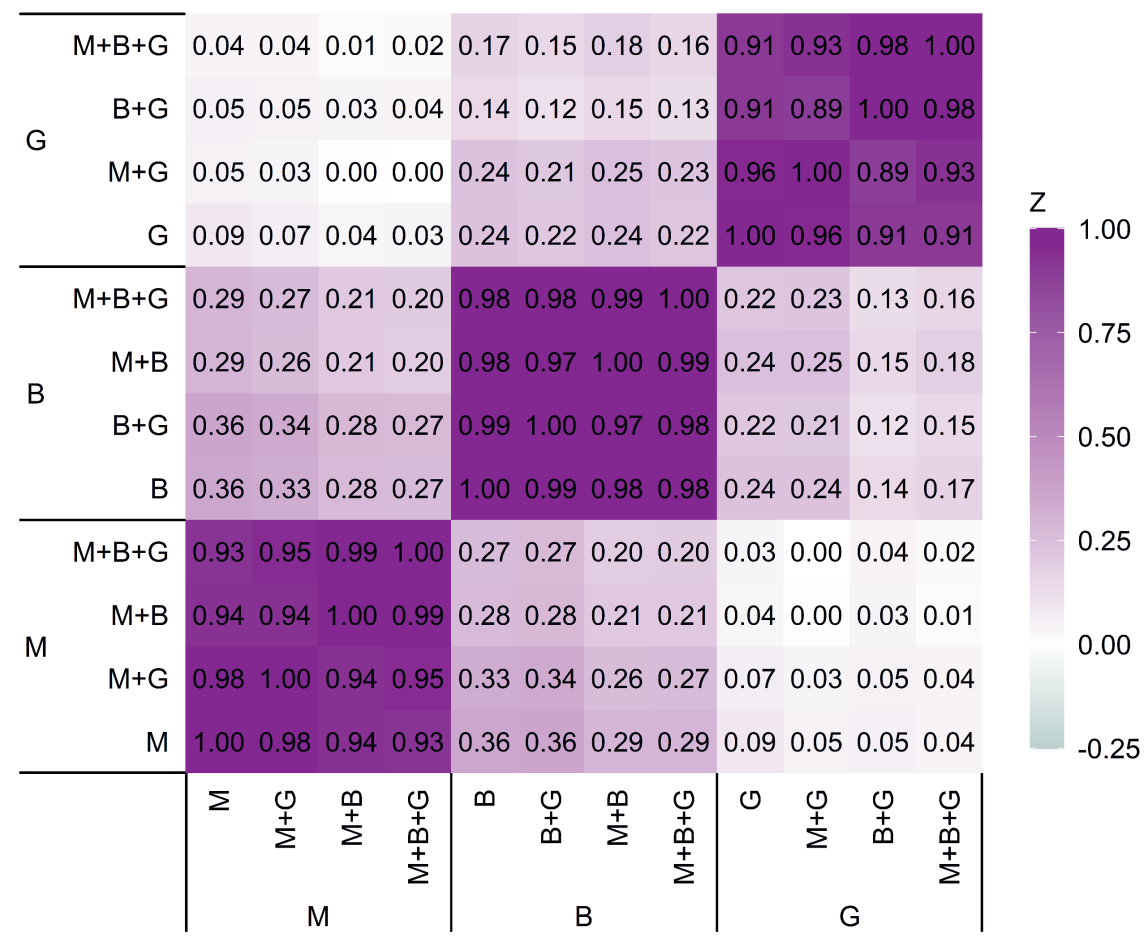

Additional file 6.4 Figure 6.S4 Heatmap of correlations between estimated effects of backfat thickness by models including microbiota $(M)$, metabolite $(B)$ and genotype $(G)$ information in different combinations 


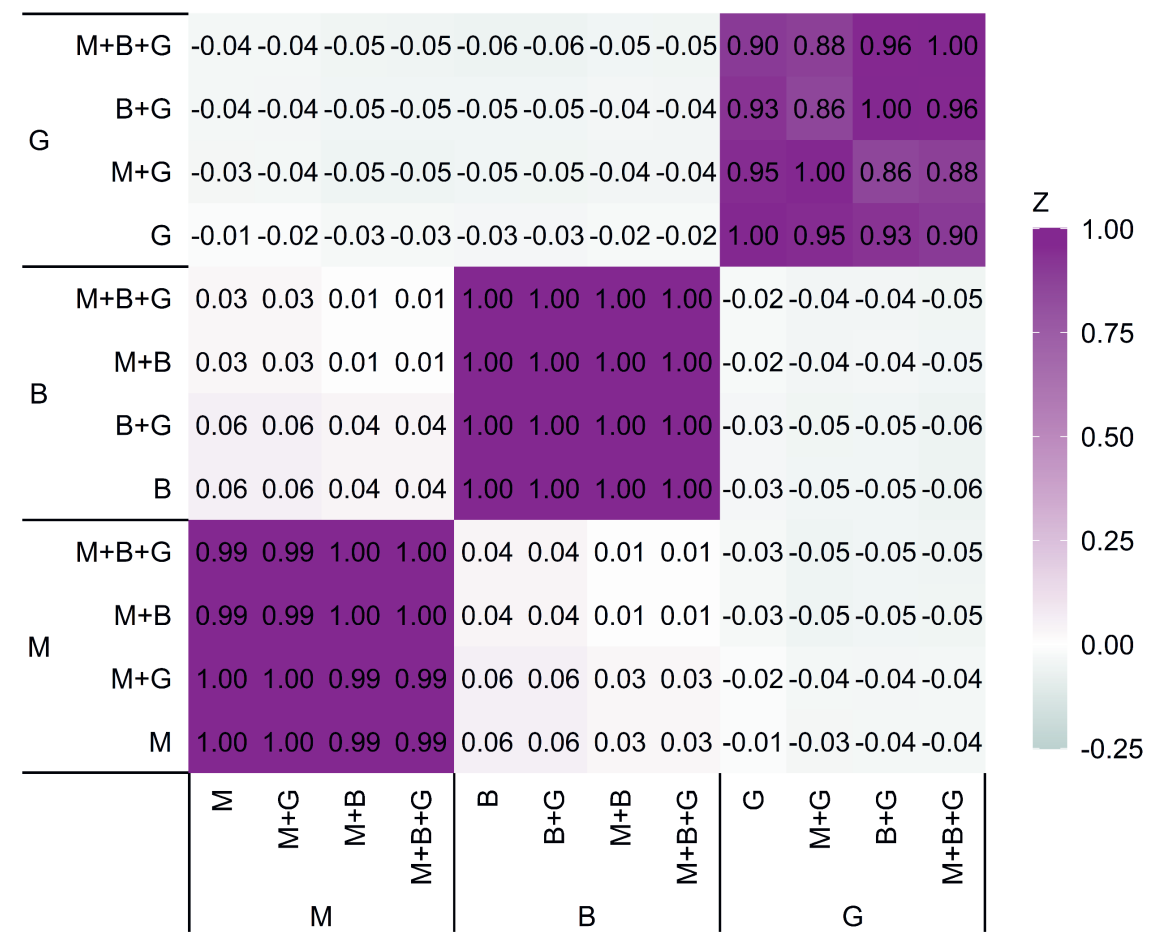

Additional file 6.5 Figure 6.55 Heatmap of correlations between estimated effects of loin depth by models including microbiota (M), metabolite (B) and genotype (G) information in different combinations

Additional file 6.6 Table 6.S1

Format: Excel

Title: Heritability of individual operational taxonomic units (OTU) and their contribution to the prediction of feed efficiency traits.

Description: ADG, average daily gain; ADFI, average daily feed intake; FCR, feed conversion ratio; RFI, residual feed intake; BF, back fat thickness; LD, loin depth.

Additional file 6.7 Table 6.S2

Format: Excel

Title: Heritability of individual metabolites and their contribution to the prediction of feed efficiency traits.

Description: ADG, average daily gain; ADFI, average daily feed intake; FCR, feed conversion ratio; $\mathrm{RFI}$, residual feed intake; $B F$, back fat thickness; $L D$, loin depth; RT, retention time; $\mathrm{mz}$, mass-to-charge ratio. 



\section{7}

General discussion 



\subsection{Introduction}

Nitrogen, carbon dioxide and methane emissions by livestock production is a worldwide problem (Steinfeld et al., 2006 ; Bodirsky et al., 2014). A small piece of the solution in reducing these emissions could come from the pig industry, as pigs are ideal animals to convert products inedible for humans to valuable protein for human consumption (Zijlstra and Beltranena, 2013). But even if pigs can use human inedible products, an inefficiency of turning dietary nutrients into body tissue of pigs leads to environmental pollution. Efficiency of turning a pig diet into a human edible product, commonly called feed efficiency, is key. Feed efficiency in grower-finisher pigs is most often expressed as kg feed per kg bodyweight gain or as the inverse of these parameters. However, looking at feed efficiency from a $\mathrm{kg}$ to $\mathrm{kg}$ perspective completely ignores the biological processes in a pig that convert dietary nutrients into body tissue. Nutrient hydrolysis by enzymes and fermentation by microbes in the gastro-intestinal tract make dietary nutrients available for absorption to the blood and lymph system. Once nutrients are absorbed, they are available for maintenance and growth of grower-finisher pigs. The balance between nutrients, immune status, physical activity, environmental temperature, and the genetic background of a pig are among the factors influencing utilization and distribution of nutrients across the pig's body. The main objective of my thesis was to investigate traits explaining variation in individual feed efficiency in pigs, and their potential to develop more effective nutritional and breeding strategies to improve feed efficiency. First, I investigated which part of the variation in individual feed efficiency is explained by variation in faecal nutrient digestibility, the faecal microbiome, and nutrient metabolism. Next, I investigated and quantified the relationship between faecal nutrient digestibility, faecal microbiome, nutrient metabolism, and host genetics. Hereinafter I will compare the results described in chapter 2 to 6 of this thesis from the perspective of pig breeding (Pilar 1: Genetics) and of pig feeding (Pilar 2: Nutrition). This will be followed by a discussion of combining knowledge in the pig breeding and feeding fields and using results of this thesis to help the breeding and feeding industry simultaneously in improving feed efficiency of grower-finisher pigs (The bridge). 


\subsection{Pilar 1: Genetics}

Take home message: Serum metabolite profiles can be used to predict phenotypes for feed efficiency traits currently used in selection, whereas faecal nutrient digestibility and nitrogen efficiency are promising new traits for genomic selection.

\subsubsection{New phenotypes}

Feed efficiency is an important trait in pig production. Large improvements in feed efficiency have been achieved by decreasing back fat thickness (BF) and improving average daily gain (ADG) of grower-finisher pigs by means of selection (Knap and Wang, 2012). However, getting a handle on biological processes not improved by selection for BF and ADG, like maintenance, could help in further improving feed efficiency of pigs (Knap and Wang, 2012). In this thesis I looked at several traits explaining variation in feed efficiency. Below I will discuss these new information sources as potential new traits for pig breeding.

\subsubsection{Microbiota}

Microbiota are small organisms living in the environment, but also inside the gastrointestinal tract of pigs. Chapter 3 described that faecal microbiota composition is related to feed efficiency of grower finisher pigs. Pigs divergent in feed efficiency could be distinguished based on their faecal microbiota composition, but only when animals were fed a high fibrous wheat/barley/by-products diet. When animals were fed a corn/soybean meal diet, the difference in faecal microbiota composition between high and low feed efficient grower-finisher pigs was not significant. This shows that faecal microbiota composition is highly sensitive to the diet fed to pigs, which has also been shown in other studies (Bauer et al., 2006; Le Sciellour et al., 2018). Faecal microbiota composition is also highly sensitive to other environmental factors, like environmental temperature (Le Sciellour et al., 2019b), antibiotic treatment (Looft et al., 2012), and geographical location (McCormack et al., 2019). Several studies have described a relationship between feed efficiency and faecal microbiota composition in pigs (Vigors et al., 2016b; Yang et al., 2017; Tan et al., 2018; McCormack et al., 2019; Bergamaschi et al., 2020; Reyer et al., 2020), yet only a couple of microbiota related to feed efficiency traits overlap across studies (Gardiner et al., 2020). 
Not only environment, but also genetics of the pig has an effect on the faecal microbiota composition. There are several levels in which microbiota composition can be expressed, but the most commonly used levels are operational taxonomic unit (OTU), which is a group of bacterial DNA strands with high similarity, or taxonomic genera. In chapter 6 it was found that some OTU are highly heritable, with values up to 0.47 . This is in line with literature, where values up to 0.50 and 0.57 were found for individual genera (Camarinha-Silva et al., 2017; Aliakbari et al., 2021). However, the microbiota described in chapter 6 that had a high contribution to the prediction of feed efficiency traits, had much lower heritability values. The average heritability of the top 10 microbiota related to feed efficiency traits ranged from 0.02 to 0.07 . Heritability of faecal microbiota could possibly be due to genetic differences in attachment sites for microbiota in the intestines, the immune response of pigs to intestinal microbiota, and processes related to nutrient digestion and uptake in the intestines. Indeed, results from Crespo-Piazuelo et al. (2019) indicate that the faecal microbiota composition is possibly influenced by pig genes related to the expression of mono and polysaccharides in the mucin of the intestinal lignin, the adaptive and innate immune response, bile acid and fatty acid metabolism, and intestinal glucose uptake. Other possible reasons for the observed heritability of faecal microbes are individual differences in the intestinal size or transit time of digesta through the gastro-intestinal tract, that are likely partly heritable themselves.

The experiment from chapter 6 was performed on one farm, and lasted for approximately one year. The diets fed to the pigs were formulated such that some variation in ingredient inclusion level occurred, but there was no variation in ingredient composition. Hence, the experiment did not have as much variation in the environment as normally experienced between or even within pig farms. As a result of the high environmental effect on the relationship between faecal microbiota composition and feed efficiency traits as shown in chapter 3 and other studies (Bauer et al., 2006; Looft et al., 2012; Le Sciellour et al., 2018; Le Sciellour et al., 2019b; McCormack et al., 2019), and the low heritability of microbes related to feed efficiency traits as shown in chapter 6 , improving feed efficiency by selecting faecal microbial species is most likely difficult.

\subsubsection{Nutrient digestibility}

Faecal nutrient digestibility is a measure of the part of nutrients consumed by the pig that are available to the pig for maintenance and growth. A lower faecal nutrient digestibility is inherent to a loss of nutrients to the environment via the faeces. Chapter 2 shows that there is substantial phenotypic variation between pigs in faecal 


\section{General discussion}

digestibility of dry matter (DM), organic matter (OM), energy, crude protein (CP), crude fat (CFat), crude fibre (CF), non-starch polysaccharides (NSP) and ash when measured at the day before slaughter and corrected for voluntary feed intake level at sampling. Part of this between animal variation in faecal nutrient digestibility could be due to genetics, as pigs with a different sire have different faecal digestibility of DM, OM, CP, and energy (Noblet et al., 2013). Indeed, Déru et al. (2021) found that heritability values of faecal energy, CP, and OM digestibility on two different diets ranged from 0.26 to 0.56 . Faecal digestibility of CFat, NSP, and ash are possibly heritable as well, as the repeatability of faecal CFat, NSP, and ash digestibility are in the same order of magnitude as estimates for DM, OM and CP digestibility (Ouweltjes et al., 2018). Many processes involved in the digestion and absorption of nutrients could be under genetic control, resulting in the heritable component of variation in faecal nutrient digestibility. Pigs divergent in RFI have different faecal digestibility of DM, energy and CP combined with differences in expression of genes related to intestinal nutrient transport, weight of the total intestinal tract and faecal microbiota composition (Vigors et al., 2016b). As shown in chapter 6, part of the faecal microbiota composition is heritable, but it is still largely unknown which biological processes cause the genetic control of faecal microbiota composition. Other possible sources of between animal variation in faecal nutrient digestibility that are possibly under genetic control are stomach acidity and muscular activity of intestinal tissue, enzyme and bile salt production, composition and activity in the small intestine, small intestinal villi length and density, and passage rate of digesta in the total gastro-intestinal tract. Nevertheless, it is known that faecal digestibility of energy, $\mathrm{CP}$, and $\mathrm{OM}$ is heritable.

Chapter 2 showed that there is a phenotypic relationship between feed efficiency traits and faecal digestibility of DM, OM, energy, CP, CFat, CF, NSP and ash. This relationship, however, changed when a pig matures, as a higher faecal nutrient digestibility of pigs up to $70 \mathrm{~kg}$ was related to having a higher ADG, whereas in pigs from 70 to $120 \mathrm{~kg}$ it was related to having a lower average daily feed intake (ADFI). In both phases the residual feed intake (RFI) was lower when the faecal nutrient digestibility of pigs was higher. Pigs require amino acids (AA) supplied through the diet in the right balance to grow body protein. If insufficient $A A$ are available or essential AA are present in an inappropriate ratio, muscle growth is hampered. Protein deposition cost energy and if insufficient energy in the form of glucose is present, glucose is formed from AA or fatty acids. Hence, the balance between AA and energy is important for efficient growth. The fraction of the sum of all AA in the diet is called (crude) protein. Although the interest is in the ileal AA digestibility and 
utilization, faecal CP digestibility is an acceptable proxy. If digestibility of a nutrient would increase, more resources are available to the pig for growth and maintenance. However, if the growth of a pig is hampered by AAs supply, an increase in fatty acid digestibility will not improve the protein deposition. Déru et al. (2021) also found a relationship between faecal nutrient digestibility and feed efficiency traits, as they showed a genetic correlation of faecal energy, CP and OM digestibility with FCR ranging from -0.16 to -0.56 . However, Déru et al. (2021) evaluated the relationship between digestibility values and feed efficiency traits over the total grower-finisher phase. This resulted in a negative genetic correlation between faecal nutrient digestibility values and both ADFI and ADG, whereas results in chapter 2 showed that the developmental stage of a pig matters for the phenotypic relationship between feed efficiency traits and faecal nutrient digestibility. Hence, the genetic correlation between faecal nutrient digestibility and feed efficiency traits should be estimated separately for the grower and finisher phase.

Although the genetic correlations of faecal energy, $C P$, and OM digestibility with ADG and ADFI were unfavourable in the study of Déru et al. (2021), both their results and the results shown in chapter 2 indicate that an increased faecal nutrient digestibility results in a favourable decrease in FCR and RFI. Balanced breeding, i.e. simultaneously selecting those traits in the desired direction, could counteract the unfavourable relationship between faecal nutrient digestibility and ADG and ADFI. Déru et al. (2021) found that heritability of faecal energy, CP, and OM digestibility depended on the composition of the diet fed, as heritability values ranged from 0.54 to 0.56 when pigs were fed a high fibre diet and from 0.26 to 0.27 when pigs were fed a conventional diet. Also the genetic variances were higher when pigs were fed a high fibre diet (Déru et al., 2021). The higher heritability and genetic variation when pigs were fed the high fibre diet suggests that feeding a high fibre diet to animals which are considered selection candidates, would result in the best selection responses. However, the genetic correlation between estimates of faecal energy, CP and $\mathrm{OM}$ on a conventional and high fibre diet were $0.71,0.85$ and 0.76 respectively (Déru et al., 2021), indicating that there is possibly an interaction between genetics and diet composition. Even when the genetic correlation is considered, selecting pigs on the high fibre diet still results in a better selection response for pigs fed the conventional diet than when selection candidates are fed the conventional diet. Thus, faecal digestibility of energy, $\mathrm{CP}$ and $\mathrm{OM}$ are interesting traits for breeding to improve feed efficiency in pigs, but selection should be balanced for correlated responses in ADG and ADFI. 


\section{General discussion}

\subsubsection{Serum metabolites}

Metabolites are produced during metabolism of nutrients and can end up in the blood immediately after digestion or after metabolism of nutrients in the body of the pig. Chapter 6 showed a high relationship between serum metabolite profile and feed efficiency traits, where metabolite profile from samples taken the day before slaughter explained 10 to $65 \%$ of the variation in feed efficiency traits measured during the whole grower-finisher period. Reyer et al. (2017) found that blood parameters indicated a shift in metabolism from energy storage towards energy utilization in animals with a low RFI. Also Horodyska et al. (2018b) found different carbohydrate and fat metabolism between high and low feed efficient pigs. When the genomic information of the pigs was included in the analysis in chapter 6 , the explained variance by blood metabolite profile did not change, but the explained variance by the genomic profile of the pig decreased. This indicates that there is some overlap in variance explained by pig genomic and blood metabolite profiles. Indeed, the metabolites that contributed most to variation in feed efficiency traits had moderate heritability values, as the top 10 metabolites contributing to the relationship had an average heritability ranging from 0.05 to 0.22 . GWAS results indicate that genes directly involved in the biological mechanisms of energy or protein metabolism were related to serum metabolite levels (Bovo et al., 2019). Serum metabolites are highly sensitive to changes, as metabolite concentrations change even within a day (Nielsen et al., 2015). The experiment described in chapter 6 was conducted in a relatively stable environment, so it is questionable if the same heritability values can also be found across animals kept in more diverse environments. Nevertheless, selection of animals for specific metabolites is likely possible and specific metabolites could be potential new traits to improve feed efficiency in grower-finisher pigs.

\subsubsection{Nitrogen efficiency}

Nitrogen efficiency is the efficiency of converting dietary nitrogen into body nitrogen. In chapter 5 a method to measure nitrogen efficiency on a large scale has been used to estimate phenotypic and genetic variation in nitrogen efficiency. Although the ratio of net energy to the concentration of SID lysine is often kept constant within a production phase, the actual AA composition of diets differs to some extent between batches of feed that is fed to pigs, due to the fact that AA composition of batches of feed ingredients are estimated and not chemically analysed. Instead of measuring $\mathrm{kg}$ feed intake compared to $\mathrm{kg}$ gain, nitrogen utilization in chapter 5 was estimated based on empirical models describing feed intake and growth curves. Ideally, AA utilization would be estimated, but this could 
not be done by the empirical models, hence we used bruto crude protein intake and protein deposition. Individual nitrogen efficiency at the first day of a new diet was estimated and ranged from 0.21 to 0.26 . The nitrogen efficiency was compared to the ADG, ADFI, and FCR over the total grower-finisher period and resulted in genetic correlations that varied between dietary phases. In the phase where the pigs were fed a starter diet, a slightly positive genetic correlation was observed between ADG and nitrogen efficiency, whereas pigs that were fed the finisher diet had a negative genetic correlation between ADG and nitrogen efficiency. Interestingly, pigs divergently selected for RFI do not show differences in nitrogen efficiency (Hewitt et al., 2020). In a nitrogen balance study where total nitrogen intake and excretion of individual pigs was measured, van der Peet-Schwering et al. (2021) showed that animals with a higher EBV for protein deposition also have a higher nitrogen efficiency. A nitrogen balance study, however, requires collection of faeces and urine of individual pigs. For animal breeding purposes, collecting qualitative faecal samples of individual pigs on a large scale is possible, but collecting urine samples is difficult. Hence, estimating nitrogen efficiency based on empirical relationships of feed intake and growth is a more suitable option for animal breeding purposes than measuring nitrogen efficiency in a nitrogen balance study, although the error of estimation is probably higher.

\subsubsection{Future prospects}

Genomic prediction is widely used in pig breeding, where based on the genome of a pig its performance later in life or the performance of its offspring can be predicted (Knol et al., 2016). To compare the potential of all possible new selection traits as discussed in this thesis to contribute to genomic improvement in the breeding program, the breeder's equation is needed,

$$
\Delta G=\frac{r_{I H} * \sigma_{G} * i}{L}
$$

in which $\Delta G$ is the improvement of a trait in one year, $r_{I H}$ is the selection accuracy, $\sigma_{G}$ is the genetic standard deviation, $i$ is the selection intensity, and $L$ is the generation interval. As the intensity of selection and generation interval is not affected by the possible new selection traits, here I assume that $\frac{i}{L}$ is equal to 1 for all traits. The $r_{I H}=$ $\sqrt{h^{2}}$ when the own performance of pigs is used for selection. In order to predict the performance of a pig based on its genome, a reference population is needed in which the performance is measured of pigs that are genetically related to pigs whose breeding values need to be predicted. When using a reference population of the same purebred, $r_{I H}$ can be estimated according to Daetwyler et al. (2008): 


\section{General discussion}

$$
r_{I H}=\sqrt{\frac{h^{2} N}{h^{2} N+M_{e}}}
$$

in which $r_{I H}$ is the selection accuracy assuming that all genetic variation is captured by the markers, $h^{2}$ is the heritability of the trait assuming the heritability is similar for purebreds and crossbreds, $N$ is the number of animals in the training population and $M_{e}$ is the number of independent chromosome segments within the population. $N$ was assumed to be 1000 purebreds, and $M_{e}$ was assumed to be 385 (Prosman, 2020). Table 7.1 shows the results of these calculations. In reality, breeding is performed on multiple traits at a time, but to compare the potential of the new traits, I assume selection on individual traits only. The genetic progress after one year of selection on only the OTU with the highest heritability will result in a change of $0.16 \%$ compared to the average of $0.32 \%$ when own performance is used and $0.18 \%$ when a reference population of 1000 animals is used. Selection for the OTU with the strongest relationship with FCR does not result in any change. Full selection on CP digestibility of a high fibre diet will result in a change in CP digestibility of $1.4 \%$ per year, whereas selection on energy digestibility on the same diet will result in change in digestibility of 1.2 to $1.3 \%$. Selection on the metabolite with highest heritability would result in intensity 1.5 times the current value, and selecting on the metabolite with highest contribution to FCR would increase intensity of that metabolite with 123 to 154 compared to an average of 2365. One year selection on nitrogen efficiency only, will result in an improvement from 55.2 to $56.8 \%$ when own performance is used and to $57.3 \%$ when a reference population of 1000 pigs is used. The genetic correlation with feed efficiency is important when looking at the potential of these candidate traits as well. Genetic correlations for OTUs with feed efficiency in chapter 6 are unknown, but the OTU with the highest contribution to FCR contributed only $0.44 \%$ to the phenotypic variation. However, Aliakbari et al. (2021) showed genetic correlations of bacterial genera with FCR as high as 0.32 . The metabolite with the highest contribution to FCR in chapter 6 contributes only $1.03 \%$ to the phenotypic variation. The genetic correlations between faecal digestibility of energy, CP and OM with FCR and RFI are $-0.16,-0.34$, and -0.21 respectively, and with $\mathrm{RFI}$ are $-0.62,-0.50$, and -0.62 respectively (Déru et al., 2021). The genetic correlation between nitrogen efficiency and FCR ranges from -0.47 to -0.90 . Hence, selection on individual OTUs and metabolites to improve FCR is likely not promising, whereas faecal energy, CP and OM digestibility, and nitrogen efficiency are traits with the highest chance of success for pig breeding. 
Table 7.1 Calculated genetic progress of optional new selection traits

\begin{tabular}{|c|c|c|c|c|c|c|c|}
\hline \multirow[b]{2}{*}{ Trait } & \multirow[b]{2}{*}{$\mu$} & \multirow[b]{2}{*}{$\sigma_{G}$} & \multirow[b]{2}{*}{$h^{2}$} & \multicolumn{2}{|c|}{$\begin{array}{l}\text { Own } \\
\text { performance }\end{array}$} & \multicolumn{2}{|c|}{$\begin{array}{l}\text { Genomic } \\
\text { selection }\end{array}$} \\
\hline & & & & $r_{I H}$ & $\Delta G$ & $r_{I H}$ & $\Delta G$ \\
\hline \multicolumn{8}{|l|}{ Microbiota } \\
\hline OTU highest $\mathrm{h}^{2}(\%)^{1}$ & 0.32 & 0.24 & 0.47 & 0.69 & 0.16 & 0.74 & 0.18 \\
\hline OTU highest FCR $(\%)^{1,2}$ & 0.00 & 0.00 & 0.03 & 0.16 & 0.00 & 0.25 & 0.00 \\
\hline \multicolumn{8}{|l|}{ Nutrient digestibility } \\
\hline Nitrogen (\%) & $73.9^{3}$ & $1.81^{3}$ & $0.56^{3}$ & 0.75 & 1.36 & 0.77 & 1.40 \\
\hline Energy (\%) & $78.2^{3}$ & $1.68^{3}$ & $0.54^{3}$ & 0.73 & 1.24 & 0.76 & 1.29 \\
\hline Organic matter (\%) & $77.9^{3}$ & $1.55^{3}$ & $0.54^{3}$ & 0.73 & 1.14 & 0.76 & 1.19 \\
\hline \multicolumn{8}{|l|}{ Nutrient metabolism } \\
\hline $\begin{array}{l}\text { Metabolite highest } h^{2} \\
\text { (intensity) }\end{array}$ & 5952 & 2832 & 0.64 & 0.80 & 2264 & 0.79 & 2237 \\
\hline $\begin{array}{l}\text { Metabolite highest FCR } \\
\text { (intensity) })^{2}\end{array}$ & 2365 & 245 & 0.25 & 0.50 & 123 & 0.63 & 154 \\
\hline Nitrogen efficiency (\%) & 55.2 & 3.43 & 0.22 & 0.47 & 1.61 & 0.60 & 2.07 \\
\hline \multicolumn{8}{|c|}{$\begin{array}{l}\mu=\text { average, } \sigma_{G}=\text { genetic variation, } h^{2}=\text { heritability, } r_{1 H}=\text { accuracy of genomic prediction, } \Delta G \\
\text { genetic progress, OTU = operational taxonomic unit, } F C R=\text { feed conversion ratio. } \\
{ }^{1} \text { Relative abundance }\end{array}$} \\
\hline
\end{tabular}

\subsubsection{Prediction tool}

\subsubsection{Predicting nutrient digestibility}

Chapter 4 described the prediction of faecal DM, OM, CP, CFat, CF, NSP and ash digestibility based on faecal microbiota composition. The prediction accuracy ranged from 0.42 for crude fibre to 0.63 for crude protein, whereas prediction of ash and crude fat digestibility was not successful. In our experiment, pigs were fed two different types of diets, a diet based on corn and soybean meal or a diet based on wheat, barley, and by-products. Diet has a profound effect on nutrient digestibility, so in order to use our prediction equations on a large scale the database on which the equations are formed should be diversified with different types of diets. Apart from diet, the environment influences faecal microbiota composition, thus making the prediction equations more universally applicable requires a diversification of the dataset with different environments and diets. Hence, improving prediction equations for nutrient digestibility based on faecal microbiota composition may be limited by the sensitivity of microbiota to dietary and environmental changes. 


\section{General discussion}

An alternative method to predict faecal nutrient digestibility is by using near-infrared spectrometry (NIRS). Labussière et al. (2019) developed a method to predict nutrient digestibility of grower-finisher pigs based on NIRS of faecal samples, with prediction accuracies of 0.93 for DM, 0.94 for OM, 0.94 for energy, 0.95 for CP, and 0.82 for CF. Déru et al. (2021) used the NIRS equations developed by Labussière et al. (2019) to predict nutrient digestibility for genetic parameter estimation. The advantages of using NIRS above microbiota to predict nutrient digestibility is that NIRS prediction equations are not as much affected by the environment. After the initial investment for a NIRS machine and developing the prediction equations, estimating nutrient digestibility is possible by only putting the sample in the machine. In comparison, predicting nutrient digestibility with microbiota composition requires DNA extraction and sequencing in the lab, which is more time consuming and expensive than using NIRS. In future, techniques could be developed that make measurement of faecal microbiota composition quick, easy and cheap. For now, however, developing NIRS equations is the most effective way to measure nutrient digestibility on large scale for pig breeding.

\subsubsection{Predicting feed efficiency traits}

Chapter 6 predicted feed efficiency traits based on faecal microbiota, serum metabolite and host genome profiles. Blood metabolite profiles gave the best phenotypic prediction (accuracies ranging from 0.42 to 0.65 ), followed by faecal microbiota profiles (accuracies $0.27-0.46$ ) and host genome profiles (accuracies $0.15-0.29$ ). Due to overlap in variation between the profiles, combining all profiles resulted in a prediction accuracy similar to only using serum metabolite profiles, indicating that blood metabolite profiles are the best to predict feed efficiency traits. However, the dataset available was from one farm only, where variation between diets in nutrient composition was limited and addition of new ingredients to the diets was prevented. In order to apply the prediction equations across several environments, the dataset should be extended to include different diets, farms, geographical locations, temperatures, health status etc. Even better, when the prediction equations will be used for phenotypic prediction of feed efficiency traits, it would be best to validate the prediction equations in the environments that are going to be predicted.

Taking blood samples is an invasive procedure that harms animal welfare, whereas taking faecal samples is less stressful to pigs. Measuring traits like average daily gain and backfat thickness are possibly as stressful to pigs as taking a faecal sample. Collecting faecal and blood samples of pigs is labour intensive and requires proper 
storage directly after collection. The lab procedures for measuring faecal microbiota composition used in chapter 6, illumina Mi-seq, requires labour intensive handlings like DNA isolation, multiplication, and sequencing. In comparison, in the methods used for serum metabolite analysis, blood samples have to be centrifuged immediately after collection, but lab analysis for LC-MS is less labour intensive than faecal microbiota analysis. In chapter 6 , specific methods to analyse the samples were used, but other procedures and techniques are available. In the future, quicker, easier and cheaper techniques to measure faecal microbiota and serum metabolite profiles might be developed. Another way to improve utility of serum metabolites or faecal microbiota for phenotypic prediction is reducing the dimension or resolution of the information, by only looking at individual or a small number of metabolites and microbiota. Most OTU and metabolites only have a small contribution to the prediction, but the top 50 contributing OTU explain 1.0 to $9.4 \%$ of the variation in feed efficiency traits, and the top 50 metabolites 1.9 to $13.7 \%$. Hence, in future techniques and methods might become available to measure serum metabolites and faecal microbiota composition, that are more suitable to use for large scale phenotypic prediction of feed efficiency traits.

\subsubsection{Improving Genomic prediction}

In chapter 6 , feed efficiency traits were predicted using the microbiota, metabolite and genome profiles simultaneously, resulting in a decreased accuracy of genomic prediction compared to a model based on genomic prediction only. The overlap between the variation explained by the different sources of information was for one trait allocated to one source and for another trait to another source. This unwanted decrease in genomic prediction accuracy when microbiota and metabolite relationship matrices are added to the prediction equations could be overcome by making the microbiota and metabolite relationship matrices conditional on the genomic relationship matrix. Making the microbiota and metabolite relationship matrices conditional on the genomic relationship matrix can be done by performing a linear regression of every OTU or metabolite on the SNP genotypes (X):

$$
\begin{gathered}
\text { OTU }_{i}=b_{0}+X b_{1}+e_{i}, \\
\text { Metabolite }_{j}=b_{0}+X b_{1}+e_{j},
\end{gathered}
$$

in which $b_{0}$ is the intercept, $b_{1}$ is the regression coefficient, and $e_{i}$ and $e_{j}$ are the residuals per OTU $\mathrm{i}$ and metabolite $\mathrm{j}$ respectively. The residuals of the OTU and metabolites are then used to form a microbiota and metabolite relationship matrix. This whole process can be done directly with matrix algebra. In the optimal case that all overlapping explained variance between the genome and metabolome or microbiome profiles would be allocated to the genome profiles, the explained 


\section{General discussion}

variances would change as shown in Table 7.2. The explained variance by microbiota profiles is then similar to the explained variance by metabolite profiles for ADG, higher for FCR and BF, and lower for ADFI and RFI. Hence, in case the microbiota and metabolite relationship matrices are made conditional on the genomic relationship matrix, either the microbiota or the metabolite relationship matrix can be used to explain environmental variance.

When the microbiota and metabolite relationship matrices are conditional on the genotype matrices, the microbiota and metabolite matrices depict their environmental effect on the phenotype. Before adding the microbiota or metabolite relationship matrix to the model, the variation in the phenotype was modelled as the sum of the variation in the genotype and the error: $\sigma_{p}^{2}=\sigma_{g}^{2}+\sigma_{\varepsilon}^{2}$. When adding the microbial relationship matrix, the formula changes, where the variation explained by microbiota comes from the error if there is no overlap between the variation explained by genotype and microbiota: $\sigma_{p}^{2}=\sigma_{g}^{2}+\sigma_{m}^{2} \overleftarrow{+\sigma}_{\varepsilon}^{2}$. Hence, the total variation explained by the genotypes does not change when adding a microbiota relationship matrix to the equation. When there is a genotype by environment interaction (GXE) for a trait of interest, the genetic variance in one environment can differ from the genetic variance in another environment. The genetic correlation between the traits in both environments is lower than one, hence selection for the trait in one environment will result in a lower selection response if progeny are housed in the other environment. As faecal microbiota composition is largely a reflection of differences in environments between pigs (chapter 6) measuring faecal microbiota composition of pigs for traits affected by GxE might aid in modelling genetic effects given a certain environment. In chapter 6 the experimental design was such that GxE was minimized. To test the theory of improved environmental quantification by adding faecal microbiota information to the model in case of GxE, a dataset of faecal microbiota and genotypes of pigs in diverse environments should be created. Preferably the dataset contains pigs fed different diets, and housed in different farms, temperatures, and geographical locations, as those factors are known to influence faecal microbiota composition. 
Table 7.2 Microbiability $\left(\mathrm{m}^{2}\right)$, metabolomability $\left(\mathrm{b}^{2}\right)$, and heritability $\left(\mathrm{h}^{2}\right)$ for feed efficiency related traits estimated by models including microbiota (M), metabolite (B) and genotype (G) relationship matrices in different combinations and where $M$ and $B$ relationship matrices are conditional on $\mathrm{G}(\mathrm{M} \mid \mathrm{G} \text { and } \mathrm{B} \mid \mathrm{G})^{1}$

\begin{tabular}{|c|c|c|c|c|c|c|}
\hline Trait $^{2}$ & $G+M$ & $G+M \mid G$ & $G+B$ & $G+B \mid G$ & $G+M+B$ & $\mathrm{G}+\mathrm{M}|\mathrm{G}+\mathrm{B}| \mathrm{G}$ \\
\hline \multicolumn{7}{|l|}{ ADG } \\
\hline $\mathrm{m}^{2}$ & 0.45 & 0.43 & - & - & 0.19 & 0.18 \\
\hline$b^{2}$ & - & - & 0.62 & 0.42 & 0.54 & 0.35 \\
\hline$h^{2}$ & 0.26 & 0.28 & 0.08 & 0.28 & 0.08 & 0.28 \\
\hline \multicolumn{7}{|l|}{ ADFI } \\
\hline $\mathrm{m}^{2}$ & 0.33 & 0.29 & - & - & 0.17 & 0.17 \\
\hline$b^{2}$ & - & - & 0.55 & 0.38 & 0.43 & 0.26 \\
\hline$h^{2}$ & 0.23 & 0.27 & 0.10 & 0.27 & 0.10 & 0.27 \\
\hline \multicolumn{7}{|l|}{ FCR } \\
\hline$m^{2}$ & 0.52 & 0.53 & - & - & 0.22 & 0.20 \\
\hline$b^{2}$ & - & - & 0.47 & 0.34 & 0.43 & 0.33 \\
\hline$h^{2}$ & 0.24 & 0.23 & 0.10 & 0.23 & 0.11 & 0.23 \\
\hline \multicolumn{7}{|l|}{ RFI } \\
\hline $\mathrm{m}^{2}$ & 0.18 & 0.17 & - & - & 0.13 & 0.12 \\
\hline$b^{2}$ & - & - & 0.32 & 0.22 & 0.26 & 0.17 \\
\hline$h^{2}$ & 0.24 & 0.25 & 0.15 & 0.25 & 0.15 & 0.25 \\
\hline \multicolumn{7}{|l|}{$\mathrm{BF}$} \\
\hline $\mathrm{m}^{2}$ & 0.39 & 0.37 & - & - & 0.29 & 0.27 \\
\hline$b^{2}$ & - & - & 0.30 & 0.16 & 0.24 & 0.11 \\
\hline$h^{2}$ & 0.32 & 0.34 & 0.20 & 0.34 & 0.19 & 0.34 \\
\hline \multicolumn{7}{|l|}{ LD } \\
\hline$m^{2}$ & 0.07 & 0.06 & - & - & 0.07 & 0.07 \\
\hline$b^{2}$ & - & - & 0.10 & 0.10 & 0.09 & 0.08 \\
\hline$h^{2}$ & 0.06 & 0.07 & 0.07 & 0.07 & 0.06 & 0.07 \\
\hline
\end{tabular}

${ }^{1}$ The expected values when $\mathrm{M}$ and $\mathrm{B}$ relationship matrices are conditional on $\mathrm{G}$, are calculated as the difference between combined estimates and estimates based on $\mathrm{G}$ alone.

${ }^{2} \mathrm{ADG}=$ average daily gain, $\mathrm{ADFI}=$ average daily feed intake, $\mathrm{FCR}=$ feed conversion ratio, $\mathrm{RFI}=$ residual feed intake, $\mathrm{BF}=$ back fat thickness, $\mathrm{LD}=$ loin depth. 


\subsection{Pilar 2: Nutrition}

Take home message: Variation in feed efficiency between pigs fed the same diet is related to differences in faecal nutrient digestion, faecal microbiota composition and serum metabolite profile, which can be measured by taking faecal and serum samples and can be used to adjust feeding strategies in precision livestock feeding.

Even when pigs are fed the exact same diet in ingredient and nutrient composition, differences between pigs in body weight gain, body composition and feed efficiency are observed. There are several reasons why pigs differ in their performance response to the exact same diet, amongst others immune status, physical activity and genetic make-up. In this thesis I looked at faecal nutrient digestibility, faecal microbiota composition, serum metabolite profile and nitrogen efficiency as possible sources of between animal variation in feed efficiency.

\subsubsection{Present}

In chapter 2 it was shown that gilts have a higher faecal digestibility of DM, OM, energy, CP, CF and NSP than boars when corrected for differences in voluntary feed intake levels. In chapter 3 it was shown that boars and gilts have a different microbiota composition as well. This difference in faecal microbiota composition can partly explain the differences in faecal digestibility between sexes, as nutrients that are not digested and absorbed in the small intestine reach the large intestine and are partly fermented by the residing microbiota. Fermentation products are absorbed across the intestinal wall and can be used in nutrient metabolism. The boars had a lower FCR and RFI than the gilts, which indicates that differences in nutrient metabolism was most likely more important to explain feed efficiency differences between boars and gilts than nutrient digestibility. Indeed, in chapter 5 it was shown that boars had a higher nitrogen efficiency than gilts.

Most digestibility trials only use one sex to determine the digestibility values of dietary ingredients, and because of practicality most often male pigs, either intact or castrated, are used in those experiments. However, by only using male pigs the average nutrient digestibility of dietary ingredients is most likely underestimated, because female pigs have higher nutrient digestibility values for dietary ingredients than boars when corrected for differences in voluntary feed intake level (chapter 2). 
As such, the overall digestibility of a diet fed to a group of pigs of both sexes is most likely underestimated. Nevertheless, it is advisable to measure nutrient digestibility of dietary ingredients on boars, castrates and gilts separately to obtain nutrient digestibility estimates for diet formulation.

The pigs in the experiments described in chapter 2, 3 and 5 were fed diets as commonly fed in Europe, a wheat/barley/by-products diet, or as fed in the Americas, a corn/soybean meal diet. The difference in faecal digestibility values and microbiota composition were consistent across the diets. Hence, it is likely that this difference between sexes can be used when formulating diets for pigs in precision livestock feeding. If the sexes would be housed and fed separately, diets could be formulated taking into account the higher faecal DM, OM, energy, CP, CF and NSP digestibility and lower nitrogen efficiency of female grower-finisher pigs. In this way the average feed efficiency of boars and gilts can be improved and environmental impact can be reduced.

\subsubsection{Near-future}

After correction for differences in faecal digestibility values between the diets and sexes, there was still substantial between animal variation in faecal nutrient digestibility (chapter 2). Part of this variation in faecal digestibility was related to feed efficiency traits. Also, the variation in faecal microbiota composition was related to feed efficiency traits after correction for dietary and sex differences (chapter 6). In chapter 5 and 6 it was shown that there is variation in nutrient metabolism between pigs of the same sex and fed the same diet. This between pig variation in faecal digestibility, faecal microbiota composition, and nutrient metabolism can most likely be used in precision livestock feeding as described hereafter.

Faecal nutrient digestibility can be measured using standard wet chemistry analysis procedures as used in digestibility trials, but a faster and cheaper method to measure faecal digestibility values has been developed: NIRS (Labussière et al., 2019). When the calibration curve to predict faecal digestibility values with NIRS will be maintained and continuously improved, measuring digestibility of individual pigs on a large scale will be possible. If the faecal digestibility values per pig can be estimated at the start of the grower-finisher phase, the pigs could be grouped based on their digestibility values. In chapter 2 the phenotypic variation in faecal digestibility of DM, OM, energy, CP, CFat, CF, NSP, and ash is described. The average faecal CP digestibility across the two diets and sexes, and corrected for differences in feed intake was $74.8 \%$. The phenotypic standard deviation for faecal CP digestibility after 


\section{General discussion}

correcting for diet, sex, interaction between diet and sex and differences in average daily feed intake was $2.6 \%$. If it is assumed that the observed phenotypic variation in faecal CP digestibility is normally distributed, can be measured with $100 \%$ accuracy, and would be divided across 4 groups of pigs, a randomisation model with 10000 observations in $\mathrm{R}$ could be used. Using this approach gives estimates of an average of $\sim 78 \%$ digestibility for the group with the highest faecal CP digestibility, $\sim 76 \%$ for the mid high group, $\sim 74 \%$ for the mid low group, and $\sim 72 \%$ for the low group. In that case, the group of pigs with the high faecal CP digestibility could be fed a diet with a $6 \%$ lower CP content than the pigs in the group with the low faecal CP digestibility, assuming that faecal CP digestibility is a good proxy for ileal AA digestibility. A similar method is used to calculate grouping based on the other nutrients discussed in chapter 2 (Table 7.3). In addition, grouping based on results from genetics study looking into faecal digestibility of energy, CP and OM (Déru et al., 2021) was investigated, and it was assumed that the accuracy of phenotypic prediction based on NIRS was equal to values estimated by Labussière et al. (2019). Table 7.3 shows the different group averages in faecal nutrient digestibility, where it is clear that the estimated difference in faecal digestibility of energy, CP, and OM between the high and low group are similar when using results from chapter 2 and Déru et al. (2021). Results of chapter 2 indicate a possible difference between the high and low group in faecal CP digestibility of $6.6 \%$, whereas pigs fed a conventional diet could be grouped with $6.2 \%$ difference, and pigs fed a high fibre diet with $5.5 \%$ difference. If cheaper diets could be used due to the higher CP digestibility, cost of the feed could be decreased. Of course, choosing more groups would result in more extreme CP digestibility values, but it would also mean more different diets would be needed.

A similar method as described above can be used to group pigs based on their ADG, $A D F I, F C R, R F I, B F$ and loin depth (LD). These traits could be predicted with faecal microbiota composition or serum metabolite profile as described in chapter 6 . Results are shown in Table 7.4, where the differences between groups are higher when feed efficiency traits are predicted with blood metabolite profiles simply because of the higher prediction accuracy. To get a grouping based on the combined traits instead of individual feed efficiency traits, economic values per feed efficiency trait should be calculated and phenotypic correlations between the traits should be estimated. By grouping pigs based on just one feed efficiency trait, pigs in the different groups could be fed more according to their predicted performance. 
Table 7.3 Group average of faecal nutrient digestibility when pigs would be grouped in four groups based on faecal digestibility of those nutrients measured with wet chemistry methods (Chapter 2) or predicted with near-infrared spectrum when pigs are fed a conventional diet (CO) or a high fibre diet (HF)

\begin{tabular}{|c|c|c|c|c|c|c|c|c|}
\hline Nutrient & $\mu$ & $\sigma_{P}$ & Acc & $\mathrm{H}$ & $\mathrm{MH}$ & $\mathrm{ML}$ & L & $\Delta \mathrm{H}-\mathrm{L}$ \\
\hline \multicolumn{9}{|l|}{ Chapter 2} \\
\hline DM & 80.8 & 1.6 & 1.00 & 82.8 & 81.3 & 80.3 & 78.7 & 4.0 \\
\hline $\mathrm{OM}$ & 82.2 & 1.7 & 1.00 & 84.4 & 82.8 & 81.7 & 80.0 & 4.3 \\
\hline Energy & 80.5 & 1.8 & 1.00 & 82.7 & 81.1 & 79.9 & 78.2 & 4.5 \\
\hline $\mathrm{CP}$ & 74.8 & 2.6 & 1.00 & 78.1 & 75.6 & 74.0 & 71.5 & 6.6 \\
\hline CFat & 78.9 & 1.9 & 1.00 & 81.3 & 79.5 & 78.3 & 76.4 & 4.9 \\
\hline $\mathrm{CF}$ & 43.1 & 6.7 & 1.00 & 51.7 & 45.3 & 40.9 & 34.6 & 17.1 \\
\hline NSP & 59.6 & 3.8 & 1.00 & 64.4 & 60.8 & 58.4 & 54.8 & 9.5 \\
\hline Ash & 50.3 & 2.9 & 1.00 & 54.0 & 51.2 & 49.3 & 46.6 & 7.4 \\
\hline \multicolumn{9}{|l|}{$\mathrm{CO}$} \\
\hline $\mathrm{CP}$ & $78.5^{1}$ & $2.7^{1}$ & $0.90^{2}$ & 81.5 & 79.3 & 77.7 & 75.4 & 6.1 \\
\hline Energy & $84.2^{1}$ & $2.1^{1}$ & $0.87^{2}$ & 86.5 & 84.8 & 83.6 & 81.9 & 4.6 \\
\hline OM & $83.4^{1}$ & $2.0^{1}$ & $0.89^{2}$ & 85.7 & 84.0 & 82.8 & 81.1 & 4.6 \\
\hline \multicolumn{9}{|l|}{$\mathrm{HF}$} \\
\hline $\mathrm{CP}$ & $73.9^{1}$ & $2.4^{1}$ & $0.90^{2}$ & 76.7 & 74.6 & 73.2 & 71.2 & 5.5 \\
\hline Energy & $78.2^{1}$ & $2.3^{1}$ & $0.87^{2}$ & 80.7 & 78.8 & 77.5 & 75.7 & 5.1 \\
\hline OM & $77.9^{1}$ & $2.1^{1}$ & $0.89^{2}$ & 80.3 & 78.5 & 77.3 & 75.5 & 4.8 \\
\hline
\end{tabular}

$\mu=$ average, $\sigma_{p}=$ phenotypic standard deviation, Acc $=$ accuracy of measurement or prediction, $\mathrm{H}=$ high group, $\mathrm{MH}=$ mid high group, $\mathrm{ML}=$ mid low group, $\mathrm{L}=$ low group, $\Delta \mathrm{H}-\mathrm{L}=$ difference between mean of the high and low group, $\mathrm{DM}=$ dry matter, $\mathrm{OM}=$ Organic matter, $\mathrm{CP}=$ crude protein, $\mathrm{CFat}=$ crude fat, $\mathrm{CF}=$ crude fibre, $\mathrm{NSP}=$ non-starch polysaccharides .

${ }^{1}$ Adapted from Déru et al. (2021).

${ }^{2}$ Adapted from Labussière et al. (2019). 


\section{General discussion}

Table 7.4 Group average of feed efficiency traits when pigs would be grouped in four groups based on feed efficiency traits as predicted with faecal microbiota composition or serum metabolite profile in chapter 6

\begin{tabular}{lllllllll}
\hline Trait & $\mu$ & $\sigma_{P}$ & $\mathrm{AcC}$ & $\mathrm{H}$ & $\mathrm{MH}$ & $\mathrm{ML}$ & $\mathrm{L}$ & $\Delta \mathrm{H}-\mathrm{L}$ \\
\hline Microbiota & & & & & & & & \\
ADG & 961 & 127 & 0.43 & 1030 & 978 & 943 & 891 & 139 \\
ADFI & 2236 & 228 & 0.46 & 2369 & 2269 & 2202 & 2103 & 266 \\
FCR & 2.35 & 0.23 & 0.27 & 2.43 & 2.37 & 2.33 & 2.27 & 0.16 \\
RFI & 0 & 136 & 0.27 & 46 & 11 & -12 & -47 & 93 \\
BF & 12 & 2.2 & 0.31 & 12.9 & 12.2 & 11.8 & 11.1 & 1.75 \\
LD & 62.2 & 7.1 & 0.05 & 62.7 & 62.3 & 62.1 & 61.7 & 0.91 \\
Metabolites & & & & & & & & \\
ADG & 961 & 127 & 0.64 & 1064 & 987 & 936 & 859 & 205 \\
ADFI & 2236 & 228 & 0.59 & 2405 & 2280 & 2193 & 2068 & 337 \\
FCR & 2.35 & 0.23 & 0.54 & 2.51 & 2.39 & 2.31 & 2.19 & 0.32 \\
RFI & 0 & 136 & 0.42 & 72 & 19 & -19 & -73 & 145 \\
BF & 12 & 2.2 & 0.45 & 13.3 & 12.3 & 11.7 & 10.8 & 2.53 \\
LD & 62.2 & 7.1 & 0.13 & 63.4 & 62.5 & 61.9 & 61.0 & 2.34 \\
\hline
\end{tabular}

$\mu=$ average, $\sigma_{p}=$ phenotypic standard deviation, Acc = accuracy of measurement or prediction, $\mathrm{H}=$ high group, $\mathrm{MH}=$ mid high group, $\mathrm{ML}=$ mid low group, $\mathrm{L}=$ low group, $\Delta \mathrm{H}-\mathrm{L}=$ difference between mean of the high and low group, $A D G=$ average daily gain, $A D F I=$ average daily feed intake, $\mathrm{FCR}=$ feed conversion ratio, $\mathrm{RFI}=$ residual feed intake, $\mathrm{BF}=$ back fat thickness, $\mathrm{LD}=$ loin depth. 
Once nutrients are digested or fermented and absorbed across the intestinal border they enter the blood readily available for the pig to use in nutrient metabolism. These blood metabolites can be measured, for example by LC-MS as used in chapter 6. Drawing a blood sample of pigs is stressful for the animal, and arguably for the animal handler, but provides information about the metabolism of pigs. Measuring a whole metabolite profile as used in chapter 6 requires high-tech equipment, but when specific metabolites are of interest easier methods could be already available, such as for creatine and free AA (van der Peet-Schwering et al., 2021), or could possibly be developed. The specific metabolites could then be used to group animals and adjust the diet nutrient composition accordingly.

The individual animal estimates for faecal nutrient digestibility or feed efficiency can also be used to adjust dietary nutrient composition on an individual animal level. Pomar and Remus (2019) nicely described how precision livestock feeding can reduce the environmental impact and improve the nutrient utilization of pigs. Remus et al. (2020) showed that using just two diets with different nutrient composition and mixing those diets to meet the individual animal's nutrient requirement real time resulted in decreased nitrogen excretion without reducing protein deposition. If individual nitrogen efficiency of pigs could be estimated real-time based on previous feed intake and bodyweight measurements, nitrogen excretion could possibly be even further reduced.

In conclusion, feeding pigs grouped based on their faecal nutrient digestibility, feed efficiency or nitrogen efficiency different diets with different nutrient composition could possibly improve feed efficiency in grower-finisher pigs. Using individual measurements to adjust nutrient composition of diets for every individual pig is also already possible due to current technological advancements in feeding equipment. 


\subsection{The bridge}

\subsubsection{Nutrition benefiting from genetics}

Take home message: Estimated breeding values for feed efficiency, but in future also nutrient digestibility, faecal microbiota composition and nutrient metabolism, could be used to predict the individual nutrient requirements of commercial crossbred pigs and adjust the nutrient composition of diets accordingly.

In this thesis it was shown that there is phenotypic variation in faecal nutrient digestibility (Chapter 2), faecal microbiota composition (chapter 3 and 6), and nutrient metabolism (chapter 5 and 6 ) that is related to variation in feed efficiency traits. Although the pig breeding industry uses variation between pigs for selection, the eventual crossbred pig raised in commercial farms should be as consistent as possible to make animal management and feeding easier and more efficient. This is what the pig breeding and animal feed industry have in common, because the animal feed industry produces diets to meet the animals' requirement and variation between pigs in their requirement makes feeding animals more complicated. However, between animal variation in the crossbred grower-finisher pigs could possibly be exploited.

Genotyping pure line pigs for breeding value estimation is the new standard in the pig breeding industry (Knol et al., 2016). Boars used to sell semen are routinely genotyped, but sows sold to multiplication farms and crossbred grower-finisher pigs are not. However, based on the genotype of the boar and sow, the average growerfinisher performance can be predicted on litter level. If crossbred piglets would also be genotyped, individual pig performance in the grower-finisher trajectory could also be predicted. In chapter 6 it was shown that genomic prediction of feed efficiency in crossbred grower-finisher pigs based on crossbred grower-finisher performance predicted phenotypes with accuracies of 0.15 to 0.29 . Although the genomic prediction accuracy is not nearly as high as predictions based on the faecal microbiota ( 0.27 to 0.46 ) or serum metabolite profiles ( 0.42 to 0.64 ), the genotype of a pig is not affected by the environment, while the microbiota and blood metabolites are. Hence, genomic prediction of crossbred grower-finisher performance could help in for example grouping pigs so that eventually all pigs in 
one pen could be delivered to the slaughterhouse at once, instead of having to pick the heaviest animals per pen.

Knowing the genomic potential of growth and feed intake of the pen of pigs could be used to adjust dietary treatments accordingly. van der Peet-Schwering et al. (2014) grouped crossbred grower-finisher pigs based on performance predicted with genomics. The prediction was based on the combined estimated breeding value (EBV) for growth, feed intake and meat percentage of sows and boars. Growerfinisher pigs with a high EBV and high birthweight were housed together and so were pigs with a low EBV and low birthweight. There were three types of feed, a control, a luxurious ( $10 \%$ more ileal digestible AA than the control diet contained) and a less luxurious diet ( $5 \%$ less ileal digestible $A A$ than the control diet contained). The high EBV grower-finisher pigs had a $65 \mathrm{~g} / \mathrm{d}$ higher ADG and a $160 \mathrm{~g} / \mathrm{d}$ higher ADFI than low EBV pigs, but the FCR was similar between the groups. The difference in net profit between the high and low EBV pigs fed the control diet was 2.50 euro. The low EBV pigs that were fed a less luxurious diet gave similar profits as those fed the control diet, but feeding the high EBV pigs a more luxurious diet did not improve profits (van der Peet-Schwering et al., 2014). When grouping pigs based on sow and boar EBV, the mendelian sampling, which is $50 \%$ of the genomic variation, is ignored and littermates cannot be distinguished from each other. The additional genomic variation due to mendelian sampling could be used to create a more accurate difference between high and low EBV pigs if individual crossbred grower-finisher pigs would be genotyped. Theoretically, the additional accuracy would lead to a $2.50 * 2$ $=5$ euro difference between groups if genomic prediction is as accurate as in the experiment of van der Peet-Schwering et al. (2014). Grouping and feeding high and low EBV pig differently did not change the profit based on the market prices at the time of the experiment (van der Peet-Schwering et al., 2014), but the reduced ileal digestible $A A$ in the diet fed to low EBV pigs reduced environmental excretion of nitrogen without reducing profit. Hence, grouping grower-finisher pigs based on their genotype could be interesting to reduce environmental pollution.

If pig breeding companies would implement recording of the new traits related to feed efficiency suggested in this thesis, faecal nutrient digestibility and nitrogen efficiency, these new traits can then also be predicted in grower-finisher pigs based on their genotypes. Grouping pigs based on genomic prediction of faecal CP digestibility requires an accurate prediction. Estimates of Déru et al. (2021) indicate a heritability $\left(h^{2}\right)$ of nitrogen digestibility of 0.27 on a conventional diet and 0.56 on a high fibre diet. As the maximum accuracy of genomic prediction of phenotypes is 


\section{General discussion}

expected to be $\sqrt{h^{2}}$ (Legarra et al., 2008), the maximum accuracy of genomic prediction of CP digestibility is 0.75 when fed a high fibre diet. Grouping growerfinisher pigs based on genomic prediction of nitrogen efficiency and faecal digestibility of $\mathrm{CP}$, energy and $\mathrm{OM}$ results in expected averages per group as shown in Table 7.5. When compared to the results shown in Table 7.3 the difference between the highest and lowest groups based on CP is decreased with $0.9 \%$, based on energy with $0.8 \%$ and based on $\mathrm{OM}$ with $0.9 \%$. Hence, grouping grower-finisher pigs based on genomic prediction results in a difference between the groups that is less than when grouping is based on predicted faecal digestibility with NIRS of the faeces of pigs. The group of pigs with a high $\mathrm{CP}$ digestibility or $\mathrm{N}$ efficiency could be fed a diet with a lower CP content than the group of pigs with a low average. Grouping pigs based on those traits predicted through genomics would only be beneficial when genotyping the pigs would be cheaper than the profit of using lower dietary protein contents.

Table 7.5 Expected group average of faecal crude protein, energy and organic matter digestibility and nitrogen efficiency when pigs would be grouped in four groups based on genomic prediction.

\begin{tabular}{|c|c|c|c|c|c|c|c|c|}
\hline Trait & $\mu$ & $\sigma_{P}$ & $h^{2}$ & $\mathrm{H}$ & $\mathrm{MH}$ & $\mathrm{ML}$ & $\mathrm{L}$ & $\Delta \mathrm{H}-\mathrm{L}$ \\
\hline \multicolumn{9}{|c|}{ Faecal nutrient digestibility } \\
\hline Crude protein (\%) & $73.9^{1}$ & $2.4^{1}$ & $0.56^{1}$ & 76.2 & 74.5 & 73.3 & 71.6 & 4.6 \\
\hline Energy (\%) & $78.2^{1}$ & $2.3^{1}$ & $0.54^{1}$ & 80.3 & 78.7 & 77.6 & 76.0 & 4.3 \\
\hline Organic matter (\%) & $77.9^{1}$ & $2.1^{1}$ & $0.54^{1}$ & 79.8 & 78.4 & 77.4 & 75.9 & 3.9 \\
\hline \multicolumn{9}{|l|}{ Nitrogen efficiency ${ }^{2}$} \\
\hline Starter phase (\%) & 53.3 & 6.6 & 0.22 & 57.2 & 54.3 & 52.3 & 49.4 & 7.8 \\
\hline Grower phase (\%) & 50.5 & 4.8 & 0.27 & 53.7 & 51.3 & 49.7 & 47.2 & 6.4 \\
\hline Finisher phase (\%) & 38.5 & 4.1 & 0.21 & 40.9 & 39.1 & 37.9 & 36.1 & 4.8 \\
\hline \multicolumn{9}{|c|}{$\begin{array}{l}\mu=\text { average, } \sigma_{p}=\text { phenotypic standard deviation, } h^{2}=\text { heritability, } H=\text { high group, } M H=\text { mid } \\
\text { high group, } M L=\text { mid low group, } L=\text { low group, } \Delta H-L=\text { difference between mean of the high } \\
\text { and low group. } \\
{ }^{1} \text { Adapted from Déru et al. (2021) of pigs fed a high fibre diet. }\end{array}$} \\
\hline
\end{tabular}




\subsubsection{Genetics benefiting from nutrition}

Take home message: Grower-finisher pigs should be fed a diet that is crude protein deficient to reliably measure nitrogen efficiency for genomic selection, and pigs should be fed a diet with high levels of fibre to increase genetic variation in current feed efficiency traits and faecal nutrient digestibility.

\subsubsection{Protein content diet}

In nutrition the balance between the building blocks for growth and maintenance of different types of tissues is essential. To gain muscle a pig needs $A A$ in the right proportions and energy to construct the AA into protein. For all cell processes in the pig's body energy is required in the form of ATP, which can be produced by the cells out of glucose and fatty-acids. If there is insufficient energy available for protein deposition, or the genetic capacity for protein deposition has been reached, or a specific amino acid is overly abundant, AA are broken down to produce glucose in the liver. In this process called gluconeogenesis, the nitrogen is detached from the AA molecule and excreted to the urine in the form of urea. The remainder of the AA molecule is then used to produce glucose or ATP. Gluconeogenesis costs ATP, hence producing glucose from AA to use in ATP production is less efficient than when dietary glucose is used. If energy is available in excess, the energy is stored in the form of lipid in fat cells. Hence, the balance between AA and energy is important for efficient nutrient utilization.

The essence of balancing dietary nutrient composition with the individual requirements of pigs is often forgotten in pig breeding, where the diets are often simply a nuisance effect corrected for in the models. Diets are formulated according to current knowledge on what an average pig needs instead of the individual selection candidate, but even if the diets would completely suit the requirements of the selection candidate to express its genetic potential, it is questionable if this is actually the best procedure to identify the most feed efficient pig. Previously the $\mathrm{kg}$ feed to kg gain approach has resulted in considerable selection responses, but to improve the feed efficiency of pigs through breeding it is most likely better to look at individual nutrients instead of the quantity of diet fed. If the aim is to improve muscle growth of pigs, it would be advisable to improve AA efficiency and energy efficiency separately, as these processes are biologically different. An animal that is efficient with $A A$ is not necessarily efficient with energy, whereas ultimately simultaneously selecting for increased efficiency of both would be best. van der 


\section{General discussion}

Peet-Schwering et al. (2021) showed that when grower-finisher pigs were fed a protein deficient diet, the average nitrogen efficiency of the pigs was $3.1 \%$ higher than when pigs were fed a protein adequate diet. When animals divergent in EBV for protein deposition were compared on the protein restricted and the protein adequate diet, the nitrogen efficiency of the low EBV pigs was lower in the protein adequate diet, whereas the nitrogen efficiency of the high EBV pigs was the same between the diets. Hence, the low EBV pigs received most likely dietary protein in excess and metabolized this protein to energy, whereas the high EBV pigs were still restricted in AA supply even when fed the protein adequate diet. Hence, growerfinisher pigs should be fed a diet that is protein deficient to reliably measure nitrogen efficiency for genomic selection.

Animals described in chapter 2 to 5 all belonged to the same experiment where pigs were fed a diet based on corn and soybean meal or wheat, barely, and by-products. In chapter 5 it was shown that indeed there are genetic differences between pigs in nitrogen efficiency, with a heritability of 0.21 to 0.27 depending on the phase. The $A A$ to lysine ratio of several AA were below the recently recommended levels in literature (Van der Peet-Schwering and Bikker, 2018). Also the lysine to net energy ratio was below the recommendations of $0.85 \mathrm{~g}$ SID lysine/MJ NE for the boars fed a grower wheat, barley, an by-products diet (Van der Peet-Schwering and Bikker, 2018). Hence, several pigs in the experiment were most likely limited in their protein deposition by dietary AA and total protein supply in at least one phase of the growerfinisher period. Chapter 2 also indicates a dietary CP restriction, as the faecal CP digestibility had the strongest phenotypic relationship with feed efficiency traits tested. In chapter 4 the results indicate that the pigs were fed a CP restricted diet as well, because the faecal microbiota composition predicted CP digestibility with an accuracy of 0.93 , which was the highest accuracy of all tested digestibility values. Intestinal microbiota are competing with each other for nutrients, hence the fastest and most efficient microbes get the best chance to ferment protein and therefore a restricted source of a dietary nutrient most likely influences the microbiota composition more than a highly abundant nutrient. The lower than recommended SID lysine to net energy ratio of the diets and the high correlation of CP digestibility with feed efficiency traits and faecal microbiota indicate that the experiment used in chapter 2 to 5 most likely fed diets restricted in protein supply.

All in all, I think the likely dietary protein restriction as unintentionally occurred in the experiment used for chapter 2 to 5 resulted in a more reliable nitrogen efficiency estimate per individual animal than when dietary protein was oversupplied. This 
more reliable nitrogen efficiency estimation contributed to finding a genetic basis for nitrogen efficiency in chapter 5 . If pig breeding companies want to improve nitrogen efficiency, feeding diets restricted in dietary protein supply would most likely give the best selection response. The level of dietary restriction to not hamper animal welfare should be carefully determined together with pig nutritionists.

\subsubsection{Fibre content diet}

Dietary composition can be fine-tuned to increase genetic variation for feed efficiency related traits between pigs. Godinho et al. (2018), using data of the same experiment as described in chapter 2 to 5 , showed that genetic variation for FCR, residual energy intake and RFI were higher on a wheat, barley and by-products based diet than on a corn and soybean meal diet, whereas the genetic variation for ADG, ADFI, average daily energy intake, protein deposition and lipid deposition were higher on a corn and soybean meal based diet. The genetic correlation between the traits on both diets were below one for FCR, RFI and residual energy intake in the starter and grower phase, but close to one when measured over the complete grower-finisher period (Godinho et al., 2018). Similarly, Déru et al. (2021) found a higher genetic variation for faecal energy, CP and OM digestibility when pigs were fed a high fibre diet compared to a conventional diet. The genetic correlations between the faecal energy, CP and OM digestibility values was $0.71,0.85$, and 0.76 respectively (Déru et al., 2021), but the genetic variation on the high fibre diet was higher to such an extent that it makes up for the low genetic correlations. Hence, selecting pigs on a high fibre diet gives better genetic progress for faecal digestibility of energy, $\mathrm{CP}$ and $\mathrm{OM}$ than selecting pigs on a conventional diet, even if progeny are fed a conventional diet. Also for variation in microbiota composition the diet is most likely important, as Camarinha-Silva et al. (2017) found microbiability values of 0.21 for FCR, 0.16 for ADFI, and 0.28 for ADG when feeding pigs a conventional diet, whereas chapter 6 of this thesis described values of $0.52,0.34$, and 0.56 for the same traits respectively when pigs were fed a diet high in fibre. Of course there were more differences between the experiment described in chapter 6 and the work of Camarinha-Silva et al. (2017), but in general faecal microbiota composition is highly related to dietary composition as also shown in chapter 3 . Hence, when the interest is in finding genetic variation between pigs in FCR, REI, RFI, faecal nutrient digestibility or faecal microbiota composition it is most likely better to feed pigs a wheat, barley, and by-products type of diet as used in the experiments described in this thesis compared to a less fibrous diet like the corn and soybean meal based diet. 


\subsection{Concluding remarks}

In this thesis I described my investigations into traits explaining variation in individual feed efficiency in pigs, and their potential to develop more effective nutritional and genetic strategies to improve feed efficiency. As this thesis relied heavily on current technological romics methods I hope that those technologies will advance and become easier and cheaper to use, so their utilization in pig breeding and feeding strategies would increase. The pig genetics companies can reach a better feed efficiency by genetic selection on faecal nutrient digestibility and nitrogen efficiency on top of selection for current feed efficiency traits. The animal feed companies producing pig diets can reach a better feed efficiency by grouping and feeding grower-finisher pigs of their clients based on the predicted performance of the pigs with methods suggested in this thesis. Individually both the breeding and feeding industry could improve feed efficiency in grower-finisher pigs, but the total pig industry could benefit from future collaborations between the breeding and feeding industry. Pig breeders could help nutritionists in predicting grower-finisher performance for precision livestock feeding and pig nutritionists could help breeders in developing a feeding strategy to express more genetic variation. Bridging the gap between genetics and nutrition is the way forward to further improve feed efficiency in grower-finisher pigs. 


\section{References}

Agudelo, J. H., M. D. Lindemann, and G. L. Cromwell. 2010. A comparison of two methods to assess nutrient digestibility in pigs. Livestock Science 133(1):7477.

Aliakbari, A., O. Zemb, Y. Billon, C. Barilly, I. Ahn, J. Riquet, and H. Gilbert. 2021. Genetic relationships between feed efficiency and gut microbiome in pig lines selected for residual feed intake. J Anim Breed Genet (1 - 17)

Arnal, M.-E., J. Zhang, S. Messori, P. Bosi, H. Smidt, and J.-P. Lallès. 2014. Early changes in microbial colonization selectively modulate intestinal enzymes, but not inducible heat shock proteins in young adult swine. PLoS One 9(2):e87967.

Baker, A. A., E. Davis, J. D. Spencer, R. Moser, and T. Rehberger. 2013. The effect of a Bacillus-based direct-fed microbial supplemented to sows on the gastrointestinal microbiota of their neonatal piglets. Journal of Animal Science 91(7):3390-3399.

Bauer, E., B. A. Williams, H. Smidt, R. Mosenthin, and M. W. Verstegen. 2006. Influence of dietary components on development of the microbiota in single-stomached species. Nutr. Res. Rev. 19(01):63-78.

Bergamaschi, M., F. Tiezzi, J. Howard, Y. J. Huang, K. A. Gray, C. Schillebeeckx, N. P. McNulty, and C. Maltecca. 2020. Gut microbiome composition differences among breeds impact feed efficiency in swine. Microbiome 8(1):110.

Bergsma, R., P. Mathur, E. Kanis, M. Verstegen, E. Knol, and J. Van Arendonk. 2013. Genetic correlations between lactation performance and growing-finishing traits in pigs. J. Anim. Sci. 91(8):3601-3611.

Bian, G., S. Ma, Z. Zhu, Y. Su, E. G. Zoetendal, R. Mackie, J. Liu, C. Mu, R. Huang, and H. Smidt. 2016. Age, introduction of solid feed and weaning are more important determinants of gut bacterial succession in piglets than breed and nursing mother as revealed by a reciprocal cross-fostering model. Environmental Microbiology 18(5):1566-1577.

Black, J., R. Campbell, I. Williams, K. James, and G. Davies. 1986. Simulation of energy and amino acid utilisation in the pig. Research and Development in Agriculture 3(3):121-145.

Bodirsky, B. L., A. Popp, H. Lotze-Campen, J. P. Dietrich, S. Rolinski, I. Weindl, C. Schmitz, C. Müller, M. Bonsch, and F. Humpenöder. 2014. Reactive nitrogen requirements to feed the world in 2050 and potential to mitigate nitrogen pollution. Nature Communications 5(1):1-7.

Bovo, S., G. Mazzoni, F. Bertolini, G. Schiavo, G. Galimberti, M. Gallo, S. Dall'Olio, and L. Fontanesi. 2019. Genome-wide association studies for 30 haematological and blood clinical-biochemical traits in Large White pigs reveal genomic regions affecting intermediate phenotypes. Sci Rep 9(1):7003.

Brestenský, M., S. Nitrayová, A. Bomba, P. Patráš, L. Strojný, V. Szabadošová, B. Pramuková, and I. Bertková. 2017. The content of short chain fatty acids in 
the jejunal digesta, caecal digesta and faeces of growing pigs. Livestock Science 205:106-110.

Buddington, R. K., C. H. Williams, B. M. Kostek, K. K. Buddington, and M. J. Kullen. 2010. Maternal-to-Infant Transmission of Probiotics: Concept Validation in Mice, Rats, and Pigs. Neonatology 97(3):250-256.

Cai, W., D. Casey, and J. C. M. Dekkers. 2008. Selection response and genetic parameters for residual feed intake in Yorkshire swine. J Anim Sci 86(2):287298.

Callahan, B. J., P. J. McMurdie, M. J. Rosen, A. W. Han, A. J. Johnson, and S. P. Holmes. 2016. DADA2: High-resolution sample inference from Illumina amplicon data. Nat Methods 13(7):581-583.

Camarinha-Silva, A., M. Maushammer, R. Wellmann, M. Vital, S. Preuss, and J. Bennewitz. 2017. Host genome influence on gut microbial composition and microbial prediction of complex traits in pigs. Genetics:1637-1644.

Camous, S., A. Prunier, and J. Pelletier. 1985. Plasma prolactin, LH, FSH and estrogen excretion patterns in gilts during sexual development. J. Anim. Sci. 60(5):1308-1317.

Caporaso, J. G., J. Kuczynski, J. Stombaugh, K. Bittinger, F. D. Bushman, E. K. Costello, N. Fierer, A. G. Pena, J. K. Goodrich, and J. I. Gordon. 2010. QIIME allows analysis of high-throughput community sequencing data. Nature methods 7(5):335-336.

Casellas, J., J. L. Noguera, J. Reixach, I. Díaz, M. Amills, and R. Quintanilla. 2010. Bayes factor analyses of heritability for serum and muscle lipid traits in Duroc pigs. J Anim Sci 88(7):2246-2254.

Castillo, M., S. M. Martín-Orúe, M. Anguita, J. F. Pérez, and J. Gasa. 2007. Adaptation of gut microbiota to corn physical structure and different types of dietary fibre. Livestock Science 109(1):149-152.

Centraal Veevoeder Bureau. 2019. Chemical compositions and nutritional values of feed ingredients. Centraal Veevoeder Bureau, Lelystad, the Netherlands.

Chen, H., X. B. Mao, L. Q. Che, B. Yu, J. He, J. Yu, G. Q. Han, Z. Q. Huang, P. Zheng, and D. W. Chen. 2014. Impact of fiber types on gut microbiota, gut environment and gut function in fattening pigs. Animal Feed Science and Technology 195:101-111.

Choct, M. 1997. Feed non-starch polysaccharides: chemical structures and nutritional significance. Feed milling international 191(June issue):13-26.

Conde-Aguilera, J. A., R. Barea, N. Le Floc'h, L. Lefaucheur, and J. Van Milgen. 2010. A sulfur amino acid deficiency changes the amino acid composition of body protein in piglets. Animal 4(8):1349-1358.

Coyte, K. Z., and S. Rakoff-Nahoum. 2019. Understanding Competition and Cooperation within the Mammalian Gut Microbiome. Current Biology 29(11):R538-R544.

Crespo-Piazuelo, D., L. Migura-Garcia, J. Estellé, L. Criado-Mesas, M. Revilla, A. Castelló, M. Muñoz, J. M. García-Casco, A. I. Fernández, and M. Ballester. 
2019. Association between the pig genome and its gut microbiota composition. Sci Rep 9(1):1-11.

Cunningham, H., D. Friend, and J. Nicholson. 1962. The effect of age, body weight, feed intake and adaptability of pigs on the digestibility and nutritive value of cellulose. Canadian Journal of Animal Science 42(2):167-175.

Daetwyler, H. D., B. Villanueva, and J. A. Woolliams. 2008. Accuracy of predicting the genetic risk of disease using a genome-wide approach. PLoS One 3(10):e3395.

De Haer, L., and A. De Vries. 1993. Feed intake patterns and feed digestibility in growing pigs housed individually or in groups. Livestock Production Science 33(3-4):277-292.

De Lange, C., J. Pluske, J. Gong, and C. Nyachoti. 2010. Strategic use of feed ingredients and feed additives to stimulate gut health and development in young pigs. Livest Sci 134(1):124-134.

den Besten, G., K. van Eunen, A. K. Groen, K. Venema, D.-J. Reijngoud, and B. M. Bakker. 2013. The role of short-chain fatty acids in the interplay between diet, gut microbiota, and host energy metabolism. J. Lipid Res. 54(9):23252340.

Déru, V., A. Bouquet, E. Labussière, P. Ganier, B. Blanchet, C. Carillier-Jacquin, and H. Gilbert. 2021. Genetics of digestive efficiency in growing pigs fed a conventional or a high-fibre diet. Journal of Animal Breeding and Genetics 138:246-258.

DeSantis, T. Z., P. Hugenholtz, N. Larsen, M. Rojas, E. L. Brodie, K. Keller, T. Huber, D. Dalevi, P. Hu, and G. L. Andersen. 2006. Greengenes, a chimera-checked $16 \mathrm{~S}$ rRNA gene database and workbench compatible with ARB. Applied and environmental microbiology 72(7):5069-5072.

Dibner, J. J., and J. D. Richards. 2005. Antibiotic Growth Promoters in Agriculture: History and Mode of Action. Poult. Sci. 84(4):634-643.

Difford, G., J. Lassen, and P. Lovendahl. 2016. Genes and microbes, the next step in dairy cattle breeding. In: EAAP, Belfast. p 285-285.

Edgar, R. C. 2010. Search and clustering orders of magnitude faster than BLAST. Bioinformatics 26(19):2460-2461.

Edgar, R. C. 2013. UPARSE: highly accurate OTU sequences from microbial amplicon reads. Nat. Methods 10(10):996-998.

Eeckhaut, V., F. Van Immerseel, E. Teirlynck, F. Pasmans, V. Fievez, C. Snauwaert, F. Haesebrouck, R. Ducatelle, P. Louis, and P. Vandamme. 2008. Butyricicoccus pullicaecorum gen. nov., sp. nov., an anaerobic, butyrate-producing bacterium isolated from the caecal content of a broiler chicken. Int. J. Syst. Evol. Microbiol. 58(12):2799-2802.

Estelle, J., C. Larzul, N. Mach, F. Levenez, Y. Ramayo-Caldas, F. Meurens, M. J. Mercat, G. Lemonnier, C. Denis, Y. Billon, I. Oswald, J. Dore, M. Berri, P. Lepage, and C. Rogel-Gaillard. 2016. Pig gut microbiota: genetic parameters and links 
with immunity traits of the host Journées de la Recherche Porcine en France No. 48. p 267-272. Institut du Porc, Paris.

Fabian, J., L. I. Chiba, D. L. Kuhlers, L. T. Frobish, K. Nadarajah, C. R. Kerth, W. H. McElhenney, and A. J. Lewis. 2002. Degree of amino acid restrictions during the grower phase and compensatory growth in pigs selected for lean growth efficiency. Journal of Animal Science 80(10):2610-2618.

Fagundes, A. C. A., R. G. Da Silva, J. D. F. Gomes, L. W. De Oliveira Souza, and R. S. Fukushima. 2009. Influence of environmental temperature, dietary energy level and sex on performance and carcass characteristics of pigs. Brazilian Journal of Veterinary Research and Animal Science 46:32-39.

Fan, P., P. Liu, P. Song, X. Chen, and X. Ma. 2017. Moderate dietary protein restriction alters the composition of gut microbiota and improves ileal barrier function in adult pig model. Scientific reports 7:43412. (Article)

Fontanesi, L. 2016. Metabolomics and livestock genomics: Insights into a phenotyping frontier and its applications in animal breeding. Animal Frontiers 6(1):73-79.

Fox, J., and S. J. T. O. Weisberg, CA. 2011. An R companion to applied regression, 2nd edn SAGE Publications.

Freire, A. C., A. W. Basit, R. Choudhary, C. W. Piong, and H. A. Merchant. 2011. Does sex matter? The influence of gender on gastrointestinal physiology and drug delivery. International Journal of Pharmaceutics 415(1-2):15-28.

Frese, S. A., K. Parker, C. C. Calvert, and D. A. Mills. 2015. Diet shapes the gut microbiome of pigs during nursing and weaning. Microbiome 3(1):28.

Frost, G., M. L. Sleeth, M. Sahuri-Arisoylu, B. Lizarbe, S. Cerdan, L. Brody, J. Anastasovska, S. Ghourab, M. Hankir, and S. Zhang. 2014. The short-chain fatty acid acetate reduces appetite via a central homeostatic mechanism. Nature Communications 5(1):1-11.

García-Gómez, E., B. González-Pedrajo, and I. Camacho-Arroyo. 2012. Role of sex steroid hormones in bacterial-host interactions. BioMed Res Int 2013

Gardiner, G. E., B. U. Metzler-Zebeli, and P. G. Lawlor. 2020. Impact of Intestinal Microbiota on Growth and Feed Efficiency in Pigs: A Review. Microorganisms 8(12):1886.

Gilmour, A. R., B. J. Gogel, B. R. Cullis, S. J. Welham, and R. Thompson. 2015. ASReml User Guide Release 4.1 Functional Specification. VSN International Ltd, Hemel Hempstead, HP1 1ES, UK.

Gloaguen, M., N. Le Floc'h, L. Brossard, R. Barea, Y. Primot, E. Corrent, and J. van Milgen. 2011. Response of piglets to the valine content in diet in combination with the supply of other branched-chain amino acids. Animal 5(11):1734-1742.

Gloaguen, M., N. Le Floc'h, E. Corrent, Y. Primot, and J. van Milgen. 2012. Providing a diet deficient in valine but with excess leucine results in a rapid decrease in feed intake and modifies the postprandial plasma amino acid and $\alpha$-keto acid concentrations in pigs. Journal of Animal Science 90(9):3135-3142. 
Godinho, R., J. Bastiaansen, C. Sevillano, F. Silva, S. Guimarães, and R. Bergsma. 2018. Genotype by feed interaction for feed efficiency and growth performance traits in pigs. Journal of Animal Science 96(10):4125-4135.

Goymann, W. 2012. On the use of non-invasive hormone research in uncontrolled, natural environments: the problem with sex, diet, metabolic rate and the individual. Methods Ecol Evol 3(4):757-765.

Haas, B. J., D. Gevers, A. M. Earl, M. Feldgarden, D. V. Ward, G. Giannoukos, D. Ciulla, D. Tabbaa, S. K. Highlander, and E. Sodergren. 2011. Chimeric 16S rRNA sequence formation and detection in Sanger and 454-pyrosequenced PCR amplicons. Genome research:494-504.

Handelsman, J. 2004. Metagenomics: Application of Genomics to Uncultured Microorganisms. Microbiology and molecular biology reviews 68(4):669685.

Harris, A., J. F. Patience, S. M. Lonergan, J. C. M. Dekkers, and N. K. Gabler. 2012. Improved nutrient digestibility and retention partially explains feed efficiency gains in pigs selected for low residual feed intake. Journal of Animal Science 90(Supplement_4):164-166.

Heger, J., T. v. Phung, and L. Krizova. 2002. Efficiency of amino acid utilization in the growing pig at suboptimal levels of intake: lysine, threonine, sulphur amino acids and tryptophan. Journal of Animal Physiology and Animal Nutrition 86(5-6):153-165.

Henry, Y., Y. Colleaux, and B. Seve. 1992. Effects of dietary level of lysine and of level and source of protein on feed intake, growth performance, and plasma amino acid pattern in the finishing pig. Journal of Animal Science 70(1):188195.

Herrmann, J., R. Hermes, and G. Breves. 2011. Transepithelial transport and intraepithelial metabolism of short-chain fatty acids (SCFA) in the porcine proximal colon are influenced by SCFA concentration and luminal $\mathrm{pH}$. Comp. Biochem. Physiol., Part A Mol. Integr. Physiol. 158(1):169-176.

Hewitt, D. J., J. C. M. Dekkers, T. Antonick, A. Gheisari, A. R. Rakhshandeh, and A. Rakhshandeh. 2020. Effects of divergent selection for residual feed intake on nitrogen metabolism and lysine utilization in growing pigs. Journal of Animal Science 98(5)

Horodyska, J., R. M. Hamill, H. Reyer, N. Trakooljul, P. G. Lawlor, U. M. McCormack, and K. Wimmers. 2019a. RNA-Seq of Liver From Pigs Divergent in Feed Efficiency Highlights Shifts in Macronutrient Metabolism, Hepatic Growth and Immune Response. Frontiers in Genetics 10(117)(Original Research)

Horodyska, J., M. Oster, H. Reyer, A. M. Mullen, P. G. Lawlor, K. Wimmers, and R. M. Hamill. 2018a. Analysis of meat quality traits and gene expression profiling of pigs divergent in residual feed intake. Meat science 137:265-274.

Horodyska, J., H. Reyer, K. Wimmers, N. Trakooljul, P. G. Lawlor, and R. M. Hamill. 2019b. Transcriptome analysis of adipose tissue from pigs divergent in feed efficiency reveals alteration in gene networks related to adipose growth, 
lipid metabolism, extracellular matrix, and immune response. Molecular Genetics and Genomics 294(2):395-408.

Horodyska, J., K. Wimmers, H. Reyer, N. Trakooljul, A. M. Mullen, P. G. Lawlor, and R. M. Hamill. 2018b. RNA-seq of muscle from pigs divergent in feed efficiency and product quality identifies differences in immune response, growth, and macronutrient and connective tissue metabolism. BMC Genomics 19(1):791.

Ingerslev, A. K., P. K. Theil, M. S. Hedemann, H. N. Lærke, and K. E. B. Knudsen. 2014. Resistant starch and arabinoxylan augment SCFA absorption, but affect postprandial glucose and insulin responses differently. $\mathrm{Br}$. J. Nutr. 111(09):1564-1576.

Jombart, T., S. Devillard, and F. Balloux. 2010. Discriminant analysis of principal components: a new method for the analysis of genetically structured populations. BMC Genet. 11(1):1.

Kemp, B., L. Den Hartog, J. Klok, and T. Zandstra. 1991. The digestibility of nutrients, energy and nitrogen in the Meishan and Dutch Landrace pig. Journal of Animal Physiology and Animal Nutrition 65:263-266.

Kim, H. B., K. Borewicz, B. A. White, R. S. Singer, S. Sreevatsan, Z. J. Tu, and R. E. Isaacson. 2011. Longitudinal investigation of the age-related bacterial diversity in the feces of commercial pigs. Vet. Microbiol. 153(1-2):124-133.

Knap, P., and L. Wang. 2012. Pig breeding for improved feed efficiency, Feed efficiency in swine. Springer. p. 167-181.

Knol, E. F., B. Nielsen, and P. W. Knap. 2016. Genomic selection in commercial pig breeding. Animal Frontiers 6(1):15-22.

Konstantinov, S. R., A. A. Awati, B. A. Williams, B. G. Miller, P. Jones, C. R. Stokes, A. D. Akkermans, H. Smidt, and W. M. de Vos. 2006. Post-natal development of the porcine microbiota composition and activities. Environ. Microbiol. 8(7):1191-1199.

Kuhl, C., R. Tautenhahn, C. Bottcher, T. R. Larson, and S. Neumann. 2012. CAMERA: an integrated strategy for compound spectra extraction and annotation of liquid chromatography/mass spectrometry data sets. Anal Chem 84(1):283289.

Labussière, E., P. Ganier, J. A. Conde, E. Janvier, and J. van Milgen. 2019. Development of a NIRS method to assess the digestive ability in growing pigs. In: 70. Annual Meeting of the European Federation of Animal Science (EAAP). p np.

Larzul, C., E. Terenina, A. Foury, Y. Billon, I. Louveau, E. Merlot, and P. Mormede. 2015. The cortisol response to ACTH in pigs, heritability and influence of corticosteroid-binding globulin. Animal 9(12):1929-1934.

Le Bellego, L., and J. Noblet. 2002. Performance and utilization of dietary energy and amino acids in piglets fed low protein diets. Livestock Production Science 76(1):45-58. 
Le Gall, M., M. Warpechowski, Y. Jaguelin-Peyraud, and J. Noblet. 2009. Influence of dietary fibre level and pelleting on the digestibility of energy and nutrients in growing pigs and adult sows. Animal 3(3):352-359.

Le Goff, G., and J. Noblet. 2001. Comparative total tract digestibility of dietary energy and nutrients in growing pigs and adult sows. Journal of Animal Science 79(9):2418-2427.

Le Naou, T., L. Floc'h, I. Louveau, H. Gilbert, and F. Gondret. 2012. Metabolic changes and tissue responses to selection on residual feed intake in growing pigs. Journal of Animal Science 90(13):4771-4780.

Le Sciellour, M., E. Labussière, O. Zemb, and D. Renaudeau. 2018. Effect of dietary fiber content on nutrient digestibility and fecal microbiota composition in growing-finishing pigs. PLoS One 13(10):e0206159.

Le Sciellour, M., D. Renaudeau, and O. Zemb. 2019a. Longitudinal analysis of the microbiota composition and enterotypes of pigs from post-weaning to finishing. Microorganisms 7(12):622.

Le Sciellour, M., O. Zemb, I. Hochu, J. Riquet, H. Gilbert, M. Giorgi, Y. Billon, J.-L. Gourdine, and D. Renaudeau. 2019b. Effect of chronic and acute heat challenges on fecal microbiota composition, production, and thermoregulation traits in growing pigs. J Anim Sci 97(9):3845-3858.

Legarra, A., C. Robert-Granié, E. Manfredi, and J.-M. Elsen. 2008. Performance of Genomic Selection in Mice. Genetics 180(1):611-618.

Lenth, R. V. 2016. Least-Squares Means: The R Package Ismeans. Journal of Statistical Software 69(1):1 - 33.

$\mathrm{Li}, \mathrm{Q}$., and J. F. Patience. 2017. Factors involved in the regulation of feed and energy intake of pigs. Animal Feed Science and Technology 233:22-33.

Liu, C., S. M. Finegold, Y. Song, and P. A. Lawson. 2008. Reclassification of Clostridium coccoides, Ruminococcus hansenii, Ruminococcus hydrogenotrophicus, Ruminococcus luti, Ruminococcus productus and Ruminococcus schinkii as Blautia coccoides gen. nov., comb. nov., Blautia hansenii comb. nov., Blautia hydrogenotrophica comb. nov., Blautia luti comb. nov., Blautia producta comb. nov., Blautia schinkii comb. nov. and description of Blautia wexlerae sp. nov., isolated from human faeces. Int. J. Syst. Evol. Microbiol. 58(8):1896-1902.

Lluch, J., F. Servant, S. Païssé, C. Valle, S. Valière, C. Kuchly, G. Vilchez, C. Donnadieu, M. Courtney, and R. Burcelin. 2015. The characterization of novel tissue microbiota using an optimized $16 \mathrm{~S}$ metagenomic sequencing pipeline. PLoS One 10(11):e0142334.

Looft, T., H. K. Allen, B. L. Cantarel, U. Y. Levine, D. O. Bayles, D. P. Alt, B. Henrissat, and T. B. Stanton. 2014. Bacteria, phages and pigs: the effects of in-feed antibiotics on the microbiome at different gut locations. ISME J 8(8):15661576.

Looft, T., T. A. Johnson, H. K. Allen, D. O. Bayles, D. P. Alt, R. D. Stedtfeld, W. J. Sul, T. M. Stedtfeld, B. Chai, J. R. Cole, S. A. Hashsham, J. M. Tiedje, and T. B. 
Stanton. 2012. In-feed antibiotic effects on the swine intestinal microbiome. Proc Natl Acad Sci U S A 109(5):1691-1696.

Mach, N., M. Berri, J. Estelle, F. Levenez, G. Lemonnier, C. Denis, J. J. Leplat, C. Chevaleyre, Y. Billon, J. Dore, C. Rogel-Gaillard, and P. Lepage. 2015. Earlylife establishment of the swine gut microbiome and impact on host phenotypes. Environ Microbiol Rep 7(3):554-569.

Magoč, T., and S. L. Salzberg. 2011. FLASH: fast length adjustment of short reads to improve genome assemblies. Bioinformatics 27(21):2957-2963.

Maltecca, C., M. Bergamaschi, and F. Tiezzi. 2020. The interaction between microbiome and pig efficiency: A review. Journal of Animal Breeding

Genetics 137(1):4-13.

Maltecca, C., D. Lu, C. Schillebeeckx, N. P. McNulty, C. Schwab, C. Shull, and F. Tiezzi. 2019. Predicting growth and carcass traits in swine using microbiome data and machine learning algorithms. Sci Rep 9(1):1-15.

Mateos, G. G., F. Martín, M. A. Latorre, B. Vicente, and R. Lázaro. 2006. Inclusion of oat hulls in diets for young pigs based on cooked maize or cooked rice. Anim. Sci. 82(1):57-63.

Mauch, E. D., J. M. Young, N. V. L. Serão, W. L. Hsu, J. F. Patience, B. J. Kerr, T. E. Weber, N. K. Gabler, and J. C. M. Dekkers. 2018. Effect of lower-energy, higher-fiber diets on pigs divergently selected for residual feed intake when fed higher-energy, lower-fiber diets. Journal of Animal Science 96(4):12211236.

McCormack, U. M., T. Curiao, S. G. Buzoianu, M. L. Prieto, T. Ryan, P. Varley, F. Crispie, E. Magowan, B. U. Metzler-Zebeli, D. Berry, O. O'Sullivan, P. D. Cotter, G. E. Gardiner, and P. G. Lawlor. 2017. Exploring a possible link between the intestinal microbiota and feed efficiency in pigs. Appl. Environ. Microbiol.

McCormack, U. M., T. Curião, B. U. Metzler-Zebeli, E. Magowan, D. P. Berry, H. Reyer, M. L. Prieto, S. G. Buzoianu, M. Harrison, and N. Rebeiz. 2019. Porcine feed efficiency-associated intestinal microbiota and physiological traits: finding consistent cross-locational biomarkers for residual feed intake. Msystems $4(4)$

McDonald, D., M. N. Price, J. Goodrich, E. P. Nawrocki, T. Z. DeSantis, A. Probst, G. L. Andersen, R. Knight, and P. Hugenholtz. 2012. An improved Greengenes taxonomy with explicit ranks for ecological and evolutionary analyses of bacteria and archaea. The ISME journal 6(3):610 - 618.

McGinnis, S., and T. L. Madden. 2004. BLAST: at the core of a powerful and diverse set of sequence analysis tools. Nucleic Acids Res. 32(suppl 2):W20-W25.

McMurdie, P. J., and S. Holmes. 2013. phyloseq: an R package for reproducible interactive analysis and graphics of microbiome census data. PLoS One 8(4):e61217.

Menon, R., S. E. Watson, L. N. Thomas, C. D. Allred, A. Dabney, M. A. Azcarate-Peril, and J. M. Sturino. 2013. Diet complexity and estrogen receptor $\beta$-status 
affect the composition of the murine intestinal microbiota. Appl. Environ. Microbiol. 79(18):5763-5773.

Meunier-Salaün, M., S. Edwards, and S. Robert. 2001. Effect of dietary fibre on the behaviour and health of the restricted fed sow. Anim. Feed Sci. Technol. 90(1):53-69.

Mevik, B.-h., and R. Wehrens. 2007. The pls Package: Principal Component and Partial Least Squares Regression in R. In: Journal of Statistical Software

Montagne, L., F. Loisel, T. Le Naou, F. Gondret, H. Gilbert, and M. Le Gall. 2014. Difference in short-term responses to a high-fiber diet in pigs divergently selected for residual feed intake. Journal of Animal Science 92(4):15121523.

Moughan, P. J., W. C. Smith, J. Schrama, and C. Smits. 1991. Chromic oxide and acidinsoluble ash as faecal markers in digestibility studies with young growing pigs. New Zealand Journal of Agricultural Research 34(1):85-88.

Navarro, D. M. D. L., E. M. A. M. Bruininx, L. de Jong, and H. H. Stein. 2019. Effects of inclusion rate of high fiber dietary ingredients on apparent ileal, hindgut, and total tract digestibility of dry matter and nutrients in ingredients fed to growing pigs. Animal Feed Science and Technology 248:1-9.

Nielsen, T. S., P. K. Theil, S. Purup, N. P. Nørskov, and K. E. Bach Knudsen. 2015. Effects of resistant starch and arabinoxylan on parameters related to large intestinal and metabolic health in pigs fed fat-rich diets. J Agric Food Chem 63(48):10418-10430.

Niu, Q., P. Li, S. Hao, Y. Zhang, S. W. Kim, H. Li, X. Ma, S. Gao, L. He, and W. Wu. 2015. Dynamic distribution of the gut microbiota and the relationship with apparent crude fiber digestibility and growth stages in pigs. Scientific reports 5:9938.

Noblet, J., H. Gilbert, Y. Jaguelin-Peyraud, and T. Lebrun. 2013. Evidence of genetic variability for digestive efficiency in the growing pig fed a fibrous diet. Animal 7(08):1259-1264.

Noblet, J., and G. Le Goff. 2001. Effect of dietary fibre on the energy value of feeds for pigs. Anim. Feed Sci. Technol. 90(1):35-52.

Noblet, J., X. Shi, C. Karege, and S. Dubois. 1993. Effets du type sexuel, du niveau d'alimentation, du poids vif et du stade physiologique sur l'utilisation digestive de l'énergie et des nutriments chez le porc: interactions avec la composition du régime. Journées de la Recherche Porcine en France 25:165-180.

Nordström, A., E. Want, T. Northen, J. Lehtiö, and G. Siuzdak. 2008. Multiple Ionization Mass Spectrometry Strategy Used To Reveal the Complexity of Metabolomics. Anal Chem 80(2):421-429.

Oksanen, J., F. G. Blanchet, M. Friendly, R. Kindt, P. Legendre, P. R. Minchin, R. B. O'Hara, G. L. Simpson, P. Solymos, M. H. H. Stevens, E. Szoecs, and H. Wagner. 2017. vegan: Community Ecology Package. 
Org, E., M. Mehrabian, B. W. Parks, P. Shipkova, X. Liu, T. A. Drake, and A. J. Lusis. 2016. Sex differences and hormonal effects on gut microbiota composition in mice. Gut Microbes 7(4):313-322.

Ouweltjes, W., L. M. G. Verschuren, J. Pijlman, R. Bergsma, D. Schokker, E. F. Knol, P. J. van der Aar, F. Molist, and M. P. L. Calus. 2018. The repeatability of individual nutrient digestibility in pigs. Livestock Science 207:63-67.

Pajarillo, E. A. B., J.-P. Chae, M. P. Balolong, H. Bum Kim, and D.-K. Kang. 2014. Assessment of fecal bacterial diversity among healthy piglets during the weaning transition. J. Gen. Appl. Microbiol. 60(4):140-146.

Paßlack, N., W. Vahjen, and J. Zentek. 2015. Dietary inulin affects the intestinal microbiota in sows and their suckling piglets. BMC veterinary research 11(1):51.

Paterson, M. J., S. Oh, and D. M. Underhill. 2017. Host-microbe interactions: commensal fungi in the gut. Current Opinion in Microbiology 40:131-137.

Pluske, J. 2016. Invited review: Aspects of gastrointestinal tract growth and maturation in the pre-and postweaning period of pigs. Journal of Animal Science 94(Supplement_3):399-411.

Pluske, J. e. a., D. Kerton, P. Cranwell, R. Campbell, B. Mullan, R. King, G. Power, S. Pierzynowski, B. Westrom, and C. Rippe. 2003. Age, sex, and weight at weaning influence organ weight and gastrointestinal development of weanling pigs. Australian Journal of Agricultural Research 54(5):515-527.

Pomar, C., and A. Remus. 2019. Precision pig feeding: a breakthrough toward sustainability. Animal Frontiers 9(2):52-59.

Prosman, A. 2020. The predicted and empirical accuracy of genomic selection in a crossbred structure using swine data. Master Thesis, Wageningen University, Unpublished.

Pruesse, E., J. Peplies, and F. O. Glöckner. 2012. SINA: Accurate high-throughput multiple sequence alignment of ribosomal RNA genes. Bioinformatics 28(14):1823-1829.

Quast, C., E. Pruesse, P. Yilmaz, J. Gerken, T. Schweer, P. Yarza, J. Peplies, and F. O. Glöckner. 2013. The SILVA ribosomal RNA gene database project: improved data processing and web-based tools. Nucleic Acids Res. 41(D1):D590-D596.

Quiniou, N., and J. Noblet. 2012. Effect of the dietary net energy concentration on feed intake and performance of growing-finishing pigs housed individually. Journal of Animal Science 90(12):4362-4372.

Ranjan, R., A. Rani, A. Metwally, H. S. McGee, and D. L. Perkins. 2016. Analysis of the microbiome: Advantages of whole genome shotgun versus $16 \mathrm{~S}$ amplicon sequencing. Biochemical

biophysical research communications 469(4):967-977.

Remus, A., L. Hauschild, M. P. Létourneau-Montminy, E. Corrent, and C. Pomar. 2020. The ideal protein profile for late-finishing pigs in precision feeding systems: Threonine. Animal Feed Science and Technology 265:114500. 
Reyer, H., M. Oster, E. Magowan, D. Dannenberger, S. Ponsuksili, and K. Wimmers. 2017. Strategies towards Improved Feed Efficiency in Pigs Comprise Molecular Shifts in Hepatic Lipid and Carbohydrate Metabolism. International journal of molecular sciences 18(8):1674.

Reyer, H., M. Oster, U. M. McCormack, E. Muráni, G. E. Gardiner, S. Ponsuksili, P. G. Lawlor, and K. Wimmers. 2020. Host-Microbiota Interactions in Ileum and Caecum of Pigs Divergent in Feed Efficiency Contribute to Nutrient Utilization. Microorganisms 8(4):563.

Roos, S., L. Engstrand, and H. Jonsson. 2005. Lactobacillus gastricus sp. nov., Lactobacillus antri sp. nov., Lactobacillus kalixensis sp. nov. and Lactobacillus ultunensis sp. nov., isolated from human stomach mucosa. Int. J. Syst. Evol. Microbiol. 55(1):77-82.

Ross, E. M., P. J. Moate, L. C. Marett, B. G. Cocks, and B. J. Hayes. 2013. Metagenomic Predictions: From Microbiome to Complex Health and Environmental Phenotypes in Humans and Cattle. PLoS One 8(9):e73056.

Sangild, P. T., A. L. Fowden, and J. F. Trahair. 2000. How does the foetal gastrointestinal tract develop in preparation for enteral nutrition after birth? Livestock Production Science 66(2):141-150.

Sankaran-Walters, S., M. Macal, I. Grishina, L. Nagy, L. Goulart, K. Coolidge, J. Li, A. Fenton, T. Williams, and M. K. Miller. 2013. Sex differences matter in the gut: effect on mucosal immune activation and inflammation. Biol Sex Differ 4(1):10.

Sarup, P., J. Jensen, T. Ostersen, O. T. Christensen, and P. Sørensen. 2018. Metabolomic phenotypic predition of growth in pigs. In: EAAP, Dbrovnik

Sauvant, D., J.-M. Perez, and G. Tran. 2004. Tables of composition and nutritional value of feed materials: pigs, poultry, cattle, sheep, goats, rabbits, horses and fish. Wageningen Academic Publishers, Wageningen, the Netherlands.

Schokker, D., J. Zhang, S. A. Vastenhouw, H. G. H. J. Heilig, H. Smidt, J. M. J. Rebel, and M. A. Smits. 2015. Long-Lasting Effects of Early-Life Antibiotic Treatment and Routine Animal Handling on Gut Microbiota Composition and Immune System in Pigs. PLoS One 10(2):e0116523.

Schop, M., A. J. M. Jansman, S. de Vries, and W. J. J. Gerrits. 2019. Increasing intake of dietary soluble nutrients affects digesta passage rate in the stomach of growing pigs. British journal of nutrition 121(5):529-537.

Self, S. G., and K.-Y. Liang. 1987. Asymptotic Properties of Maximum Likelihood Estimators and Likelihood Ratio Tests under Nonstandard Conditions. Journal of the American Statistical Association 82(398):605-610.

Sevillano, C. A., C. V. Nicolaiciuc, F. Molist, J. Pijlman, and R. Bergsma. 2018. Effect of feeding cereals-alternative ingredients diets or corn-soybean meal diets on performance and carcass characteristics of growing-finishing gilts and boars. Journal of Animal Science 96(11):4780-4788. 
Shirali, M., P. Varley, and J. Jensen. 2017. Longitudinal genetic dissection of feed efficiency and feeding behaviour in MaxGro pigs. Livestock Science 199:7985.

Short, F. J., P. Gorton, J. Wiseman, and K. N. Boorman. 1996. Determination of titanium dioxide added as an inert marker in chicken digestibility studies. Animal Feed Science and Technology 59(4):215-221.

Slavica, A., A. Trontel, N. Jelovac, Ž. Kosovec, B. Šantek, and S. Novak. 2015. Production of lactate and acetate by Lactobacillus coryniformis subsp. torquens DSM 20004T in comparison with Lactobacillus amylovorus DSM 20531T. J. Biotechnol. 202:50-59.

Smith, C. A., E. J. Want, G. O'Maille, R. Abagyan, and G. Siuzdak. 2006. XCMS: processing mass spectrometry data for metabolite profiling using nonlinear peak alignment, matching, and identification. Anal Chem 78(3):779-787.

Steinfeld, H., P. Gerber, T. Wassenaar, V. Castel, M. Rosales, M. Rosales, and C. de Haan. 2006. Livestock's long shadow: environmental issues and options. Food \& Agriculture Org.

Tan, Z., Y. Wang, T. Yang, H. Ao, S. Chen, K. Xing, F. Zhang, X. Zhao, J. Liu, and C. Wang. 2018. Differences in gut microbiota composition in finishing Landrace pigs with low and high feed conversion ratios. Antonie van Leeuwenhoek:1-13.

Thompson, C. L., B. Wang, and A. J. Holmes. 2008. The immediate environment during postnatal development has long-term impact on gut community structure in pigs. The ISME journal 2(7):739-748.

Urriola, P. E., and H. H. Stein. 2012. Comparative digestibility of energy and nutrients in fibrous feed ingredients fed to Meishan and Yorkshire pigs. Journal of Animal Science 90(3):802-812.

Van Der Kloet, F. M., I. Bobeldijk, E. R. Verheij, and R. H. Jellema. 2009. Analytical error reduction using single point calibration for accurate and precise metabolomic phenotyping. J Proteome Res 8(11):5132-5141.

Van der Peet-Schwering, C., and P. Bikker. 2018. Amino acid requirement of growing and finishing pigs, Wageningen UR Livestock Research.

van der Peet-Schwering, C., L. Troquet, G. Binnendijk, R. Vogelzang, and E. F. Knol. 2014. Voerstrategie voor vleesvarkens in relatie tot genetische aanleg en geboortegewicht. 1570-8616, Wageningen UR Livestock Research.

van der Peet-Schwering, C. M. C., L. M. G. Verschuren, R. Bergsma, M. S. Hedemann, G. P. Binnendijk, and A. J. M. Jansman. 2021. The effects of birth weight and estimated breeding value for protein deposition on nitrogen efficiency in growing pigs. J Anim Sci

Van Kernebeek, H. R., S. J. Oosting, M. K. Van Ittersum, P. Bikker, and I. J. De Boer. 2016. Saving land to feed a growing population: consequences for consumption of crop and livestock products. Int J Life Cycle Assess 21(5):677-687. 
van Milgen, J., A. Valancogne, S. Dubois, J.-Y. Dourmad, B. Sève, and J. Noblet. 2008. InraPorc: a model and decision support tool for the nutrition of growing pigs. Animal Feed Science Technology 143(1-4):387-405.

VanRaden, P. M. 2008. Efficient methods to compute genomic predictions. J Dairy Sci 91(11):4414-4423.

Verschuren, L. M. G., M. P. L. Calus, A. J. M. Jansman, R. Bergsma, E. F. Knol, H. Gilbert, and O. Zemb. 2018. Fecal microbial composition associated with variation in feed efficiency in pigs depends on diet and sex. J Anim Sci 96(4):1405-1418.

Verschuren, L. M. G., D. Schokker, R. Bergsma, A. J. M. Jansman, F. Molist, and M. P. L. Calus. 2020. Prediction of nutrient digestibility in grower-finisher pigs based on faecal microbiota composition. J Anim Breed Genet 137(1):23-35.

Vigors, S., J. V. O'Doherty, A. K. Kelly, C. J. O'Shea, and T. Sweeney. 2016a. The Effect of Divergence in Feed Efficiency on the Intestinal Microbiota and the Intestinal Immune Response in Both Unchallenged and Lipopolysaccharide Challenged Ileal and Colonic Explants. PLoS One 11(2):e0148145.

Vigors, S., T. Sweeney, C. J. O'Shea, A. K. Kelly, and J. V. O'Doherty. 2016b. Pigs that are divergent in feed efficiency, differ in intestinal enzyme and nutrient transporter gene expression, nutrient digestibility and microbial activity. Animal 10:1848-1855.

Wellock, I., P. Fortomaris, J. Houdijk, J. Wiseman, and I. Kyriazakis. 2008. The consequences of non-starch polysaccharide solubility and inclusion level on the health and performance of weaned pigs challenged with enterotoxigenic Escherichia coli. Br. J. Nutr. 99(03):520-530.

Wen, C., W. Yan, C. Sun, C. Ji, Q. Zhou, D. Zhang, J. Zheng, and N. Yang. 2019. The gut microbiota is largely independent of host genetics in regulating fat deposition in chickens. The ISME journal

Weng, M., and W. Walker. 2013. The role of gut microbiota in programming the immune phenotype. Journal of developmental origins of health disease 4(3):203-214.

Wenk, C. 2001. The role of dietary fibre in the digestive physiology of the pig. Animal Feed Science and Technology 90(1):21-33.

Wigmore, P., and N. Stickland. 1983. Muscle development in large and small pig fetuses. Journal of anatomy 137(Pt 2):235.

Wilfart, A., J. van Milgen, J. Noblet, L. Montagne, and P. H. Simmins. 2007. Sites of nutrient digestion in growing pigs: Effect of dietary fiber. Journal of Animal Science 85(4):976-983.

Williams, B. A., M. W. Verstegen, and S. Tamminga. 2001. Fermentation in the large intestine of single-stomached animals and its relationship to animal health. Nutr. Res. Rev. 14(02):207-228.

Xiao, L., J. Estellé, P. Kiilerich, Y. Ramayo-Caldas, Z. Xia, Q. Feng, S. Liang, A. $\varnothing$. Pedersen, N. J. Kjeldsen, and C. Liu. 2016. A reference gene catalogue of the pig gut microbiome. Nature Microbiology 1:16161. 
Yang, H., X. Huang, S. Fang, M. He, Y. Zhao, Z. Wu, M. Yang, Z. Zhang, C. Chen, and L. Huang. 2017. Unraveling the fecal microbiota and metagenomic functional capacity associated with feed efficiency in pigs. Frontiers in microbiology 8:1555.

Zamaratskaia, G., J. Babol, A. Madej, E. J. Squires, and K. Lundström. 2004. Agerelated Variation of Plasma Concentrations of Skatole, Androstenone, Testosterone, Oestradiol-17 $\beta$, Oestrone Sulphate, Dehydroepiandrosterone Sulphate, Triiodothyronine and IGF-1 in Six Entire Male Pigs. Reprod. Domest. Anim. 39(3):168-172.

Zelezniak, A., S. Sheridan, and K. R. Patil. 2014. Contribution of Network Connectivity in Determining the Relationship between Gene Expression and Metabolite Concentration Changes. PLOS Computational Biology 10(4):e1003572.

Zhao, J., Q. Wang, L. Liu, Y. Chen, A. Jin, G. Liu, K. Li, D. Li, and C. Lai. 2018. Comparative digestibility of nutrients and amino acids in high-fiber diets fed to crossbred barrows of Duroc boars crossed with Berkshirex Jiaxing and Landracex Yorkshire. Asian-Australasian journal of animal sciences 31(5):721.

Zhou, L., L. Fang, Y. Sun, Y. Su, and W. Zhu. 2016. Effects of the dietary protein level on the microbial composition and metabolomic profile in the hindgut of the pig. Anaerobe 38:61-69.

Zijlstra, R., and E. Beltranena. 2013. Swine convert co-products from food and biofuel industries into animal protein for food. Animal Frontiers 3(2):48-53. 
Summary 


\section{Summary}

\section{Summary}

The efficiency of turning a pig diet into a human edible product, commonly called feed efficiency, is key to decrease environmental pollution of the pig industry. In this thesis I bridge the genetics and nutrition expertise fields in pig production. I investigate traits explaining variation in individual feed efficiency in pigs, and their potential to develop more effective nutritional and breeding strategies to improve feed efficiency.

Chapter 2 describes the variation in feed efficiency traits of grower-finishers pigs associated with the variation in faecal digestibility values, independent of variation in feed intake. Variation in faecal digestibility of nutrients is related to variation in average daily gain, back fat thickness, feed conversion ratio and residual feed intake in younger animals, whereas in older animals faecal digestibility of nutrients was related to variation in average daily feed intake and residual feed intake. Hence, the relationship between variation in feed efficiency traits and faecal nutrient digestibility is different across the developmental stages of a pig.

Chapter 3 investigates the association between feed efficiency and faecal microbial composition in commercial grower-finisher pigs. In addition, two factors affecting feed efficiency are investigated for their effect on the faecal microbiome: diet composition and sex. There are differences in faecal microbiota composition between high and low feed efficient grower-finisher pigs, but only when pigs are fed a fibre rich diet based on wheat, barley and by-products and not if the diet is based on corn and soybean meal. Microbiota related to feed efficiency are different between male and female pigs. Hence, there is a diet and sex dependent relationship between feed efficiency and the faecal microbiota composition in grower-finisher pigs.

Chapter 4 estimates the proportion of phenotypic variance in faecal digestibility explained by differences in individual faecal microbiota composition (i.e. the microbiability). In addition, the accuracy of predicting faecal digestibility based on microbiota composition is evaluated. Microbiability values for faecal digestibility of dry matter, organic matter, crude protein, crude fibre, and non-starch polysaccharides are moderate to high. The accuracies of prediction are relatively low if the interest is in precisely predicting faecal nutrient digestibility of individual pigs, but are promising from the perspective of ranking animals in a genetic selection context. 
Chapter 5 describes the phenotypic variation among grower-finishers pigs for individual nitrogen and amino acid efficiency predicted based on feed intake and growth curves, and estimates the heritability of nitrogen efficiency and its genetic correlation with feed efficiency traits. Variation in amino acids between pigs is substantial and nitrogen efficiency has a moderate heritability. The phenotypic and genetic correlation between nitrogen efficiency and feed efficiency traits changes over time. Selection for increased nitrogen efficiency is likely possible but will result in decreased feed conversion ratio by reducing average daily feed intake and average daily gain.

Chapter 6 investigates the contribution of host genome, faecal microbiota composition, and concentrations of serum metabolites to variation in feed efficiency traits. The proportion of phenotypic variance in feed efficiency traits associated with microbiota and metabolite profiles is identified, the accuracy of prediction of feed efficiency phenotypes using microbiota and metabolite profiles is evaluated, and the heritability of the faecal microbiome and serum metabolome is estimated to investigate their relationship with host genome. Results show that combining information on the genotype of the pig and its faecal microbiota and blood metabolite profiles improves the accuracy of prediction of phenotypes for feed efficiency related traits, but almost the same prediction accuracy can be achieved using blood metabolite profiles only.

Finally, I discuss the implications of the findings described in this thesis for the pig breeding and feeding industry in chapter 7. Pig breeding companies can reach a better feed efficiency by genetic selection on faecal nutrient digestibility and nitrogen efficiency on top of selecting for current feed efficiency traits. The companies producing pig diets can reach a better feed efficiency by grouping and feeding grower-finisher pigs of their clients based on the expected performance of the pigs, that can be predicted from faecal microbiota composition, serum metabolite profiles or faecal nutrient digestibility. Individually, both the breeding and feeding industry could improve feed efficiency in grower-finisher pigs, but the pig industry can benefit from future collaborations between the breeding and feeding industries. Pig breeders could help nutritionists in predicting grower-finisher performance for precision livestock feeding and pig nutritionists could help breeders in developing a feeding strategy to express more genetic variation. Bridging the gap between genetics and nutrition is the way forward to further improve feed efficiency in grower-finisher pigs. 

Résumé 


\section{Résumé}

\section{Résumé}

L'efficacité de transformation d'une alimentation porcine en un produit comestible pour l'homme, communément appelée efficacité alimentaire, est essentielle pour réduire la pollution environnementale de l'industrie porcine. Dans cette thèse, je fais le lien entre les domaines d'expertise génétique et nutritionnelle en production porcine. J'étudie les caractéristiques expliquant la variation de l'efficacité alimentaire individuelle chez les porcs, et leur potentiel pour développer des stratégies nutritionnelles et génétiques plus efficaces pour améliorer l'efficacité alimentaire.

Le chapitre 2 décrit la variation de l'efficacité alimentaire des porcs en croissancefinition associée à la variation des valeurs de digestibilité fécale, indépendamment de la variation de la prise alimentaire. La variation de la digestibilité fécale des nutriments est liée à la variation du gain quotidien moyen, de l'épaisseur de lard dorsal, du taux de conversion alimentaire et de la prise alimentaire résiduelle chez les animaux plus jeunes, tandis que chez les animaux plus âgés, la digestibilité fécale des nutriments est liée à la variation de la prise alimentaire quotidienne moyenne et de la prise alimentaire résiduelle. Par conséquent, la relation entre la variation de l'efficacité alimentaire et la digestibilité des nutriments fécaux est différente selon les stades de développement d'un porc.

Le chapitre 3 étudie l'association entre l'efficacité alimentaire et la composition microbienne fécale chez les porcs commerciaux en croissance-finition. De plus, deux facteurs affectant l'efficacité alimentaire sont étudiés pour leur effet sur le microbiome fécal: la composition du régime alimentaire et le sexe. Des différences dans la composition du microbiote fécal existent entre les porcs en croissancefinition à haute et faible efficacité alimentaire, mais uniquement lorsque les porcs reçoivent un régime riche en fibres à base de blé, d'orge et de sous-produits et non si le régime est à base de maïs et de tourteau de soja. Le microbiote lié à l'efficacité alimentaire est différent entre les porcs mâles et femelles. Par conséquent, une relation dépendante du régime alimentaire et du sexe existe entre l'efficacité alimentaire et la composition du microbiote fécal chez les porcs en croissancefinition. 
Le chapitre 4 estime la proportion de la variance phénotypique de la digestibilité fécale expliquée par les différences de composition individuelle du microbiote fécal (c'est-à-dire la microbabilité). De plus, la précision de la prédiction de la digestibilité fécale basée sur la composition du microbiote est évaluée. Les valeurs de microbabilité pour la digestibilité fécale de la matière sèche, de la matière organique, des protéines brutes, des fibres brutes et des polysaccharides non amylacés sont modérées à élevées. Les précisions de prédiction sont relativement faibles si l'intérêt est de prédire avec précision la digestibilité des nutriments fécaux de porcs individuels, mais sont prometteuses du point de vue du classement des animaux dans un contexte de sélection génétique.

Le chapitre 5 décrit la variation phénotypique chez les porcs en croissance-finition pour l'efficacité individuelle de l'azote et des acides aminés prédite sur la base de la consommation alimentaire et des courbes de croissance, et estime l'héritabilité de l'efficacité de l'azote et sa corrélation génétique avec les caractéristiques de l'efficacité alimentaire. La variation des acides aminés entre les porcs est importante et l'efficacité de l'azote a une héritabilité modérée. La corrélation phénotypique et génétique entre l'efficacité de l'azote et les caractères d'efficacité alimentaire changent au fil du temps. La sélection pour une efficacité accrue de l'azote est probablement possible, mais entraînera une diminution du taux de conversion alimentaire en réduisant la prise alimentaire quotidienne moyenne et le gain quotidien moyen.

Le chapitre 6 étudie la contribution du génome de l'hôte, de la composition du microbiote fécal et des concentrations de métabolites sériques à la variation des traits d'efficacité alimentaire. La proportion de variance phénotypique dans les caractéristiques de l'efficacité alimentaire associée aux profils de microbiote et de métabolites est identifiée, la précision de la prédiction des phénotypes de l'efficacité alimentaire à l'aide des profils de microbiote et de métabolites est évaluée et I'héritabilité du microbiome fécal et du métabolome sérique est estimée pour étudier leur relation avec le génome hôte. Les résultats montrent que la combinaison d'informations sur le génotype du porc et ses profils de microbiote fécal et de métabolites sanguins améliore la précision de la prédiction des phénotypes pour les caractères liés à l'efficacité alimentaire, même si une précision de prédiction presque similaire peut être obtenue en utilisant uniquement les profils de métabolites sanguins. 


\section{Résumé}

Enfin, je discute des implications des résultats décrits dans cette thèse pour l'industrie de l'élevage et de l'alimentation des porcs au chapitre 7. Les entreprises d'élevage de porcs peuvent atteindre une meilleure efficacité alimentaire grâce à la sélection génétique sur la digestibilité des nutriments fécaux et l'efficacité de l'azote en plus de la sélection pour les caractéristiques de l'efficacité actuelle. Les entreprises produisant des aliments pour porcs peuvent atteindre une meilleure efficacité alimentaire en regroupant et en nourrissant les porcs en croissance-finition de leurs clients en fonction des performances attendues des porcs, qui peuvent être prédites à partir de la composition du microbiote fécal, des profils de métabolites sériques ou de la digestibilité des nutriments fécaux. Individuellement, l'industrie de l'élevage et de l'alimentation pourrait améliorer l'efficacité alimentaire des porcs en croissance-finition, mais l'industrie porcine peut bénéficier de futures collaborations entre les industries de l'élevage et de l'alimentation. Les éleveurs de porcs pourraient aider les nutritionnistes à prédire les performances des porcs en croissance-finition pour une alimentation précise du bétail et les nutritionnistes pourraient aider les éleveurs de porcs à développer une stratégie d'alimentation pour exprimer plus de variation génétique. Combler le fossé entre la génétique et la nutrition est la voie à suivre pour améliorer encore l'efficacité alimentaire des porcs en croissance-finition. 
Curriculum Vitae 


\section{Curriculum Vitae}

\section{About the author}

Lisanne Verschuren was born on the $19^{\text {th }}$ of November in 1990 in Veghel, The Netherlands. She received her VWO degree from Gymnasium Bernrode in HeeswijkDinther. Lisanne grew up at a pig farm and was always interested in the biology of life. Biology was one of her favourite subjects at high school and her graduation project was about learning behaviour in pigs. Hence, she chose to start a bachelor in Animal Sciences at Wageningen University in 2009. Her BSc thesis was about microbiota in caesarean born piglets. After obtaining her BSc degree in Wageningen she continued to do a MSc in Animal Sciences at Wageningen University. The first year of her Masters she specialised in animal breeding and genetics, where she conducted her MSc thesis about total feed efficiency in pigs at IPG (nowadays Topigs Norsvin Research Center B.V.). She continued to study and specialised in animal nutrition by entering the European Master in Sustainable Animal Nutrition and Feeding. During this specialisation Lisanne studied at both Wageningen University in the Netherlands and Aarhus University in Denmark. During her internship at Nutreco she performed research in China and investigated the ileal digestibility of two different fibre sources in grower-finisher pigs. She continued her studies with an MSc thesis about nutrient metabolism in lactating sows and developed a computer model to estimate nutrient flows within the body of lactating sows. After handing in her MSc thesis in 2015, she started to work for Topigs Norsvin as a junior researcher. In mid-2016, Lisanne started her PhD at Wageningen University and Agrocampus Ouest, in collaboration with Topigs Norsvin, to work on the Feed-a-Gene project. She was attracted by the broad spectrum of nutritional and genetic related subjects under investigation by the multitude of private and public partners involved in the European project. In 2016 and 2019 she visited the lab of Olivier Zemb at INRAe in Toulouse for two months, and in 2017 she visited the lab of Mette Skou Hedemann at Aarhus University in Foulum for one month. Additionally, she went to collaborate with Jaap van Milgen at INRAe in Rennes for three months in 2018 and 2019. Lisanne currently works full-time as a junior researcher at Topigs Norsvin in Beuningen. 


\section{Peer reviewed publications}

Verschuren, L.M.G., Schokker, D., Bergsma, R., Jansman, A.J.M., Molist, F., \& Calus, M.P.L. (2020). Prediction of nutrient digestibility in grower-finisher pigs based on faecal microbiota composition. Journal of Animal Breeding and Genetics, 137(1), 23-35.

Verschuren, L.M.G., Calus, M.P.L., Jansman, A.J.M., Bergsma, R., Knol, E.F., Gilbert, H., \& Zemb, O. (2018). Fecal microbial composition associated with variation in feed efficiency in pigs depends on diet and sex. Journal of Animal Science, 96(4), 1405-1418.

Verschuren, L.M.G., Schokker, D., Bergsma, R., van Milgen, J., Molist, F., Calus, M.P.L., \& Jansman, A.J.M. Variation in faecal digestibility values related to feed efficiency traits of grower-finisher pigs. Animal (accepted).

Verschuren, L.M.G., Jansman, A.J.M, van Milgen, J., Zemb, O., Hedemann, M.S., Bergsma, R., \& Calus, M.P.L. Combining faecal microbiome, serum metabolome, and host genome for phenotypic prediction of feed efficiency in grower-finisher pigs. Submitted.

Van der Peet-Schwering, C.M., Verschuren, L.M.G., Bergsma, R., Hedemann, M.S., Binnendijk, G.P., \& Jansman, A.J.M. (2021). The effects of birth weight and estimated breeding value for protein deposition on nitrogen efficiency in growing pigs. Journal of Animal Science, 99(6), skab101.

Zemb, O., Achard, C. S., Hamelin, J., De Almeida, M. L., Gabinaud, B., Cauquil, L., Verschuren, L.M.G., \& Godon, J. J. (2020). Absolute quantitation of microbes using 16S rRNA gene metabarcoding: A rapid normalization of relative abundances by quantitative PCR targeting a 16S rRNA gene spike-in standard. MicrobiologyOpen, 9(3), e977.

van der Peet-Schwering, C.M., Verschuren, L.M.G., Hedemann, M.S., Binnendijk, G.P., \& Jansman, A.J.M. (2020). Birth weight affects body protein retention but not nitrogen efficiency in the later life of pigs. Journal of Animal Science, 98(6), skaa180.

Ouweltjes, W., Verschuren, L.M.G., Pijlman, J., Bergsma, R., Schokker, D., Knol, E. F., van der Aar, P.J., Molist, F., \& Calus, M.P.L. (2018). The repeatability of individual nutrient digestibility in pigs. Livestock Science, 207, 63-67.

Bergsma, R., Verschuren, L. M. G., \& Knol, E. F. (2018). 29 Can We Select for an Improved Microbiome?. Journal of Animal Science, 96(suppl_2), 15-16. 


\section{Contributions to conferences}

Verschuren, L. M.G, Jansman, A. J. M., Zemb, O., Bergsma, R., \& Calus, M. P. L. (2020). Microbiability of feed efficiency related traits in pigs. The 71th EAAP conference, online.

Verschuren, L.M.G. (2020). Potential role of the microbiome in a modern pig breeding program to increase animal production. Animal Microbiome \& Nutritional Health Congress, Chicago, USA

Verschuren, L. M. G., Calus, M. P. L., Schokker, D., Bergsma, R., Molist, F., \& Jansman, A. J. M. (2019). Looking at nutrition related traits in pigs through genetic-tinted glasses. The 70th EAAP conference, Ghent, Belgium.

Verschuren, L. M. G., Schokker, D., Jansman, A. J. M., Bergsma, R., Molist, F., \& Calus, M. P. L. (2019). Microbiability of faecal nutrient digestibility in pigs. The 70th EAAP conference, Ghent, Belgium.

Verschuren, L.M.G., Jansman, A.J.M., Calus, M.P.L., Bergsma, R. \& Hedemann, M.S. (2018). Plasma metabolites related to nitrogen efficiency in low and high birthweight pigs. The 14th DPP Symposium, Brisbane, Australia.

Verschuren, L.M.G., Jansman, A.J.M., Bergsma, R., Molist, F., Calus \& M.P.L., Schokker, D. (2018). The relationship between fecal nutrient digestibility and microbiota composition in grower-finisher pigs at slaughter weight. The 14th DPP Symposium, Brisbane, Australia.

Verschuren, L.M.G, Ouweltjes, W., Pijlman, J., Bergsma, R., Schokker, D., Knol, E. F., van der Aar, P.J., Molist, F., \& Calus, M.P.L. (2018). Sources of variation in individual fecal nutrient digestibility in pigs. The 14th DPP Symposium, Brisbane, Australia.

Verschuren, L. M. G., Calus, M. P., Jansman, A. J. M., Bergsma, R., Knol, E. F., Gilbert, H., \& Zemb, O. (2017). Feed efficiency and the faecal microbiome at slaughter weight in pigs. The 68th EAAP conference, Tallinn, Estonia. 
Training and education

\begin{tabular}{|ll|}
\hline The Basic Package (3.0 ECTS) \\
\hline WIAS Introduction Day & 2016 \\
Course on philosophy of science and/or ethics & 2017 \\
Course on essential skills & 2017 \\
\hline
\end{tabular}

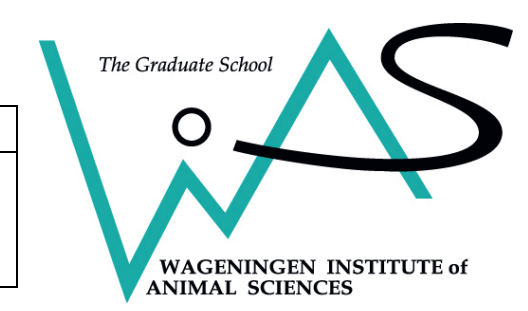

\begin{tabular}{|lr|}
\hline Disciplinary Competences (21.6 ECTS) & \\
\hline Writing own research proposal & 2016 \\
Getting started in AS-REML & 2016 \\
Genomics (Wageningen) & 2016 \\
Research skills training INRA & 2016 \\
Epigenesis and epigenetics (Wageningen) & 2017 \\
Biological consequences of selection (Norway) & 2017 \\
Design of Breeding Programs with Genomic Selection (Wageningen) & 2017 \\
Statistics for the life sciences (Wageningen) & 2018 \\
\hline
\end{tabular}

\begin{tabular}{|lr|}
\hline Professional Competences (4.9 ECTS) & \\
\hline Stress Identification \& Management & 2017 \\
Scientific Writing & 2018 \\
Effective behaviour in your professional surroundings & 2019 \\
Project and time management & 2019 \\
Competence assessment & 2019 \\
\hline
\end{tabular}

\begin{tabular}{|lr|}
\hline Presentation Skills (maximum of 4.0 ECTS) & \\
\hline European Association of Animal Production (EAAP) - Oral & 2017 \\
International symposium - Digestive Physiology of Pigs - Oral + Poster & 2018 \\
European Association of Animal Production (EAAP) - 2 x Oral & 2019 \\
European Association of Animal Production (EAAP) - Oral & 2020 \\
\hline
\end{tabular}

\section{Teaching competences (1.5 ECTS)}

Teaching in course (Nutrient Absorption and Metabolism, Aarhus University) 2016

Total: 35.0 ECTS 

Acknowledgements 


\section{Acknowledgements}

This thesis would not have been possible without all the help I received from all possible sides. Five years of hard work by all people involved, who have struggled and celebrated together with me.

First of all, I would like to thank my supervisors, who have supported me through the good and the bad; not only times, but also analysing, writing, presenting, publishing and all the other activities involved in growing and learning as an academic student. Mario, you were always available to help me out, even if my bad planning lead to emails during the night, weekend or holidays. No statistical or mathematical problem was too difficult for you to solve, and your innovative mind came up with solutions I had never thought of. You kept me on track of the larger goal of my thesis and tried to real my ambitions in a bit. Alfons, you made sure I would not forget the biology behind all the statistical and mathematical approaches, and that I would keep the practical application in mind. Sometimes I read our publications and I wonder how we came up with such nice sentences. And then I think of you, because you brought my writing to the next level. I liked to do things a bit differently, and you tried to keep me to the status quo. Sometimes you managed, sometimes I was stubborn. Jaap, you helped me think outside the box and together we had the wildest plans and ambitions. We would start with a small idea, and by the end of the meeting I had more papers to write and analyses to perform than I could ever manage within the time I had for my thesis. Luckily there is life after a PhD. Rob, you helped in organizing and executing the experiments and everything around it. Without you, I would not have had any data to work with. We spend lots of time together traveling to and joining in meetings all over Europe, to show our experimental designs and results. You made sure that I could focus on my thesis and enjoy the academic life as much as possible. Having four different supervisors from different institutes and disciplines within one project was sometimes challenging, but I think I can proudly say that the disciplines and institutes have come closer together during this project. At the beginning of the project we spoke different academic languages, and by now we understand each other. I am not $100 \%$ sure we bridged the gap completely, but we are getting closer.

Next, I would like to thank my friends and colleagues. During these years I spend a couple of months a year at different locations, mostly in France. When I was in the Netherlands, I spend half my time at Wageningen University and the other half at Topigs Norsvin. Everywhere I went, I felt very welcomed and connected to the people. Roos, if there is one person who helped me through personal and emotional struggles, it is you. I can't thank you enough for brightening things up and keeping 
my feet on the ground when I needed it. Biaty, lunches with you were always a good reminder of what is important in life and how to get most out of opportunities. You helped me understand complex genetic theories and made it look simple. Charlotte, your positive spirit and endless energy has been a real inspiration. Your approach to life, enjoying it and giving everything you have, is what made my time in Rennes memorable. Egbert, you were always there when I needed you, and you always challenged me and helped me think deeper and further than I could ever achieve on my own. When I struggled with writing during the last months of my thesis you and Annelies opened up your house for me to work in. Thank you and Annelies for providing me with a motivational surrounding when I needed it the most. I would also like to thank my other colleagues from Topigs Norsvin. Barbara, Rodrigo, Claudia, Louisa, Marleen, Marcos, Jenelle, Martijn, Victor, Susan, Chris and Catalin, thank you for your challenges, ideas, support, and help. Ilene, Paul, Teng, Jette, and Imke, thank you for all the hard work in the pig farm, without you I had no data to do my thesis! Also thank you to all the people at Wageningen University, you created a welcoming and fun atmosphere to be in, I always looked forward to work in Wageningen. A big thank you to my friends Sasha, Ying, Denise, Farran, Bijie, Alberto, Matthieu, Mihai, and Mireille, because our holidays and fun conversations were the best moments of the year that gave me renewed energy to continue my thesis. No holiday or conference was complete without you guys, and I hope we can continue for many years to come!

Last, but not least, a huge thank you to my partner and family. Nick, we met each other when I was in the first year of my thesis, and since the day we met you never failed to support me. You convinced me I could conquer the world and made me realize that the small achievements are important too. You gave me a warm and welcoming home from which I travelled the world. Dad, without your help and support my horses and I would not have survived. You allowed me back into your house when I started my thesis, fed my horses when I was abroad, and never complained about the extra work and mess it gave. Afterwards you helped me with my own house, and later you again helped Nick and me with our new house. I can always count on you, thank you so much for everything. Tim, thank you for filling in when dad was not available. Dionne, thank you for listening to my struggles during my thesis and helping me figure out which steps to take. Bea, Willie, and Liza, thank you for believing in me as well, you accepted me in your family as your own, and I could not have wished for a more supportive and warm family to be part of And the rest of my family, thank you for all the (unasked) advices and nice parties, they kept me going with a positive spirit. 


\section{Colophon}

The research described in this thesis was financially supported by Topigs Norsvin Research Center B.V. (Beuningen, The Netherlands) and the Feed-a-Gene project, which received funding from the European Union's Horizon 2020 Research and Innovation Program under the grant agreement no. 633531. It was co-funded by the Dutch Ministry of Economic Affairs (TKI Agri \& Food project 12039; Public-private partnership "Breed\&Feed4Food” code BO-22.04-010-001).

The cover of this thesis was designed by Fabiënne van Berkum.

The thesis was printed by DigiForce | Proefschriftmaken.nl 

a

$6^{.0}, 0.74$

$10^{\circ} \quad 0^{\circ}$.

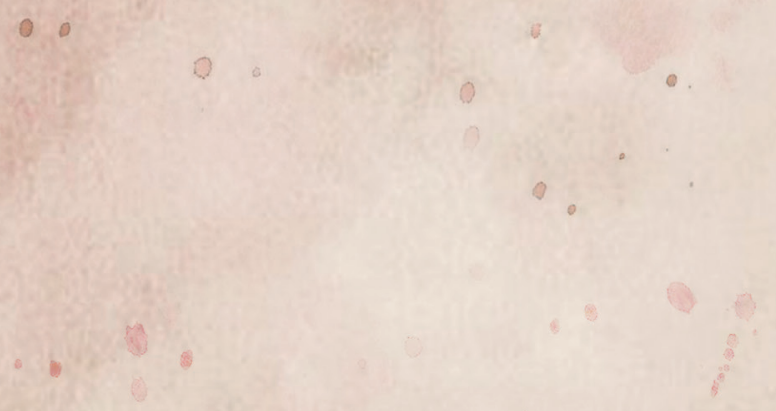

fo. . .

(1)
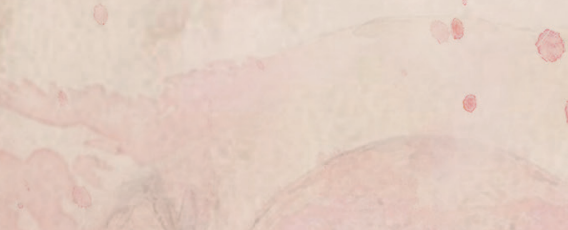

-

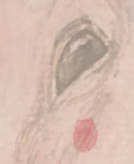

7

8
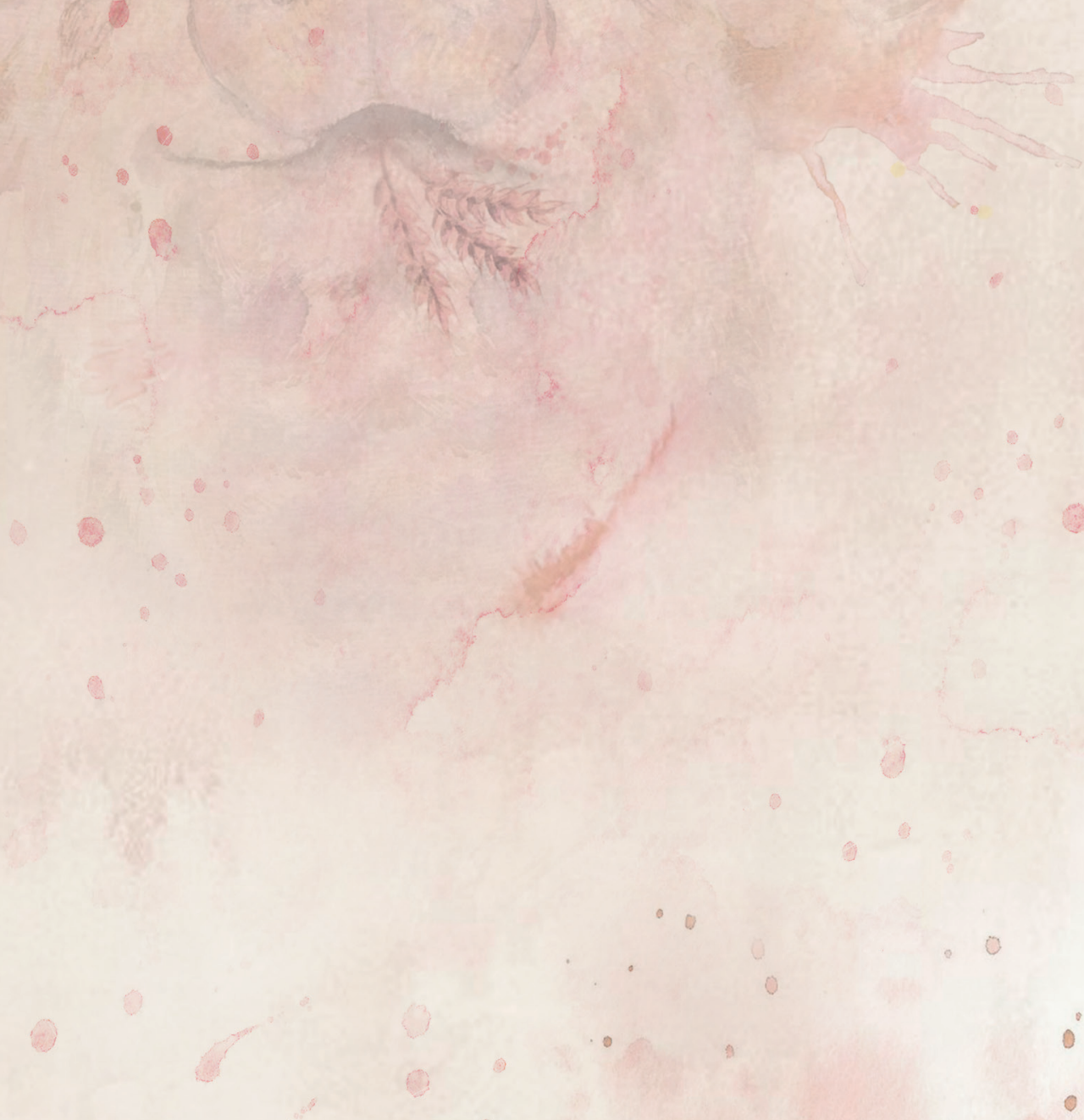